\section{Article}

Mycosphere

Copyright $\odot 2013$
Online Edition

Doi 10.5943/mycosphere/4/3/2

\title{
Smut fungi of Iran
}

\section{Vánky $K^{1}$ and Abbasi $M^{2}$}

\author{
${ }^{1}$ Herbarium Ustilaginales Vánky (HUV), Gabriel-Biel-Str. 5, D-72076 Tübingen, Germany \\ ${ }^{2}$ Iranian Research Institute of Plant Protection, Department of Botany, P.O. Box 1454, Tehran 19395, Iran
}

Vánky K, Abbasi M 2013 - Smut fungi of Iran. Mycosphere 4(3), 363-454, Doi 10.5943/mycosphere/4/3/2

\begin{abstract}
A short history of the knowledge of Iranian smut fungi is given followed by an account of the 99 known smut fungus species (Ustilaginomycetes) from Iran. Each species is presented with its authors, place of publication, synonyms, description, host plants and geographic distribution. A key to the 16 genera, to which these smuts belong, and keys to the species within each genus are given. There is also a host plant - smut fungus index. The following six species are known only from Iran: Anthracoidea songorica, Entyloma majewskii, Tilletia rostrariae, Tranzscheliella iranica, Urocystis behboudii and Urocystis phalaridis.
\end{abstract}

Key words - Biodiversity - Iran - parasitic microfungi - smut fungi - synonyms Ustilaginomycetes

\section{Introduction}

\section{A short history of the knowledge of the Iranian smut fungi}

Mycology in Iran started in 1830 with the report of Parmelia esculenta (Goebel 1830). Thirty years later Buhse (1860) published a comprehensive paper about plants, lichens and fungi of Transcaucasia and Persia. He reported 33 species of fungi from this area, but no smut. The first smut fungus, Tilletia sorghi (= Sporisorium sorghi) was reported on Sorghum sp. by Rabenhorst (1871). Wettstein (1885) reported Ustilago ornithogali (= Vankya heufleri) on Tulipa kerneri from Ghazvin area. Urocystis orobanches was reported on Phelipaea sp. from Bushehr Province, southern Iran (Magnus 1893). Magnus (1899) published four smut fungi from the south east of Persia (Kerman province), among them a new species, Ustilago lanigeri Magnus (= Sporisorium lanigeri) on Andropogon laniger. Bornmüller (1908) published an article about the flora of Alborz Mountain, and he reported one smut species viz. Ustilago ischaemi (= Sporisorium andropogonis) on Andropogon ischaemum from Gachsar and Taleghan. Sydow \& Sydow (1908) reported Ustilago vaillantii (= Antherospora tourneuxii) on Bellevalia glauca from several localities in western Iran. González Fragoso (1916) reported Sphacelotheca ischaemi on Andropogon hirtum var. pubescens from Gotvand, in south west of Iran. Petrak \& Esfandiari (1941) reported the following smut fungi, mainly from Central Alborz: Cintractia caricis on Carex sp., Sphacelotheca andropogonis on Andropogon ischemum, Sph. holci-sorghi on Sorghum halepense, Sph. schweinfurthiana on Imperata cylindrica, Tilletia hordei on Hordeum leporinum, Ustilago crameri on Setaria viridis, and U. cynodontis on Cynodon dactylon. Esfandiari (1946) reported 14 smut species from different parts of the country. This was the first publication in Persian language (Farsi) about smut fungi. Esfandiari 
(1948) published a paper describing the smut fungi of cereals in Iran. A new species, Tuburcinia behboudii (= Urocystis behboudii) was described on Arrhenatherum kotschyi from Iran (in Esfandiari \& Petrak 1950). Esfandiari (1951), in his fourth list about Iranian fungi, reported four additional smut fungi. Khabiri $(1952,1956,1958)$ published three lists about Iranian fungi. In the second list six smut species, and in the third list three smut species were enumerated. ViennotBourgin (1958) published 13 smut fungi from Iran. He also described a new species, Tuburcinia ranunculi-muricati (= Urocystis ranunculi) on Ranunculus muricatus (Viennot-Bourgin 1968). Jørstad (1960) mentioned two smut fungi from Iran viz. Cintractia caricis (= Anthracoidea sp.) on Carex diluta, and Ustilago ornithogali (= Vankya ornithogali) on Gagea chomutovae. Smut fungi on various cereals were published by Niemann \& Bamdadian (1967), Mehrian (1984), Pourjam (1989), Sharifnabi \& Hedjaroude (1993) and Torabi et al. (1996). Viennot-Bourgin et al. (1969) enumerated 13 smut fungi from Iran. Viennot-Bourgin et al. (1970) mentioned two smut fungi new to Iran, Entyloma australe and Tuburcinia ixiolirii (= Urocystis ixiolirii). Ershad (1977) published the first edition of Fungi of Iran. He listed 34 smut species. Vánky \& Ershad (1993) reported three genera and 21 species of smut fungi new to Iran. Ershad (1994) enumerated six new smut fungi to Iran and made a new combination, Sporisorium penniseti (Rabenh.) Ershad. Ershad (1998 ('1997')) reported a new smut fungus to Iran and made the new combination Sporisorium lanigeri (Magnus) Ershad. Ershad (2000) proposed the new genus Vankya for some smut fungi on Liliaceae, based on the type species, V. ornithogali. In a comprehensive paper, Ershad (2001) published (in Farsi) 76 smut fungi from Iran belonging to 13 genera, giving also drawings of the infected plants as well as LM pictures of the spores. After Ershad's monograph, several new records of smut fungi were published by different authors, e.g. Tilletia bornmuelleri and Urocystis fischeri (Hedjaroude \& Abbasi 2000), Anthracoidea angulata and A. pratensis (Kukkonen \& Ershad 2002), A. caryophylleae and A. tomentosae (Ershad \& Amini-Rad 2003), and A. michelii (Ershad \& Amini-Rad 2004). Vánky \& Ershad (2002) described a new Tilletia (T. rostrariae) from Iran. Ershad (2003) reported Urocystis ornithogali from Iran. Vánky et al. 2011('2010') published five smut fungi new to Iran.

Remarks - Host plants and geographic distribution in Iran are mainly based on herbarium specimens, deposited in the Fungal Reference Collection of the Ministry of Jihad-e Agriculture (IRAN) and in Herbarium Ustilaginales Vánky (HUV). Localities without indication of herbaria are taken from the literature. They are usually common smuts.

\section{Key to the genera}

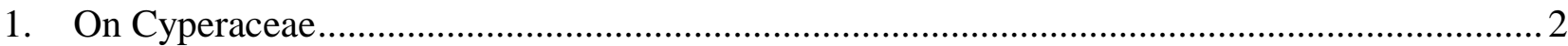

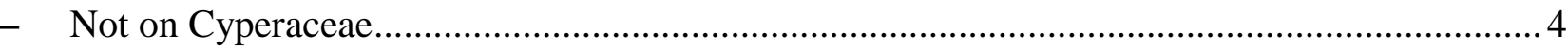

2. Sori on leaves forming striae; spores in pairs agglutinated in balls. .........................Schizonella

- Sori in flowers or around nuts; spores single ................................................................ 3

3. Sori in flowers, olivaceous brown, powdery, with fungal filaments ............................. Farysia

- $\quad$ Sori around the nuts, black, hard, without fungal filaments .................................Anthracoidea

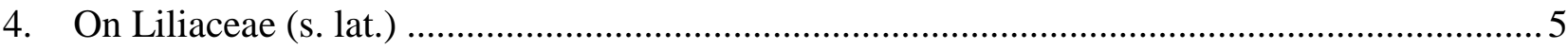

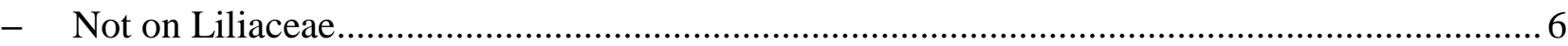

5. Sori on the leaves as pustules; indistinct sterile cells present among the spores............ Vankya

- Sori in the flowers; sterile cells absent ..................................................................Antherospora

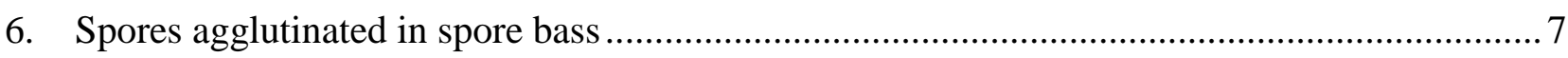

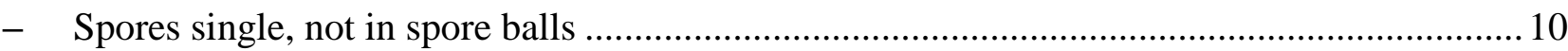

7. Spore balls formed of spores and sterile fungsl cells or hyphae ....................................... 8

- Spore balls formed of spores only (when mature often single) ................................Sporisorium

8. Spore balls formed of an outer layer of spores and a central mass of hyphae................. Tracya

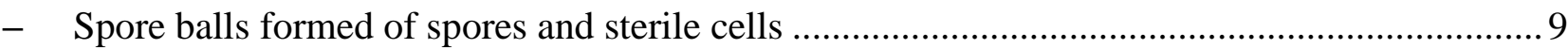

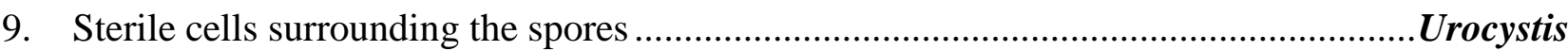


- Sterile cells scattered among the spores

10. Spores embedded in the host tissue, not powdery

- Spores not embedded in the host tissue, powdery

11. Spores subhyaline or pale yellowish brown Entyloma

- Spores olivaceous or blackish brown Jamesdicksonia

12. Sori on the surface of stems or floral axis; spores usually small $(<8 \mu \mathrm{m})$ Tranzscheliella

- Sori not so; spores usually larger 13

13. Spore mass and spores violet tinted; on dicots (various families) Microbotryum

- Spore mass and spores not violet tinted; on monocots (Poaceae) 14

14. Basidium one-celled (holobasidium). Tilletia

- Basidium several-celled (phragmobasidium) 15

15. Spores mixed with sterile cells, spores single or in groups Macalpinomyces Spores not mixed with sterile cells, spores single Ustilago

\section{Antherospora R. Bauer, M. Lutz, Begerow, Piątek \& Vánky}

\section{Key to the Antherospora species}

1. Spores 7-15(-17.5) $\mu \mathrm{m}$ long; on Bellevalia A. tourneuxii

- Spores 6.5-12(-14) $\mu \mathrm{m}$ long; on Muscari .... A. vaillantii

1. Antherospora tourneuxii (A.A. Fisch. Waldh.) R. Bauer, M. Lutz, Begerow, Piątek \& Vánky, in Bauer, Lutz, Begerow, Piątek, Vánky, Bacigálová \& Oberwinkler, Mycol. Res. 112: 1302, 2008. Ustilago vaillantii Tul. \& C. Tul. var. tourneuxii A.A. Fisch. Waldh. 1880. — Ustilago tourneuxii (A.A. Fisch. Waldh.) Maire 1931b. - Yenia tourneuxii (A.A. Fisch. Waldh.) T.N. Liou 1949. Sori in all deformed, globoid flowers of an inflorescence, in the anthers and on the surface of inner floral organs, producing blackish brown, powdery spore mass, for a long time enclosed by the outermost floral envelopes. Spores ovoid, ellipsoidal, elongate, often bent, usually irregular, rarely subglobose, $6.5-8 \times 7-15(-17.5) \mu \mathrm{m}$, yellowish brown; wall even, c. $0.5 \mu \mathrm{m}$ thick, finely, densely punctate-verruculose, spore profile smooth to finely wavy (Fig. 1).

On Hyacinthaceae (Liliaceae s. 1.):

Bellevalia glauca (Lindl.) Kunth, Markazi Prov., Arak, Sefidkhane Mountain; Lorestan Prov., Shahu Mountain.

Bellevalia saviczii Woronow, Kermanshah Prov., $17 \mathrm{~km}$ SE of Paveh city, S slope of Shahu Mt., alt. 2200 m, 15.V.2006, M. Abbasi, R. Fritsch \& M. Keusgen, IRAN 14782 F, HUV 21824.

2. Antherospora vaillantii (Tul. \& C. Tul.) R. Bauer, M. Lutz, Begerow, Piątek \& Vánky, s. lat., in Bauer, Lutz, Begerow, Piątek, Vánky, Bacigálová \& Oberwinkler, Mycol. Res. 112: 1304, 2008.

Ustilago vaillantii Tul. \& C. Tul. 1847. - Yenia vaillantii (Tul. \& C. Tul.) Liou 1949. - Vankya vaillantii (Tul. \& C. Tul.) Ershad 2000.

Ustilago vaillantii forma muscari Rabenh. 1876 (nom. nud).

Ustilago muscari-botryoidis Cif. 1928.

Sori in all, slightly swollen flowers of an inflorescence, in the anthers and on the filaments, rarely also on gynoecium and in the ovaries, filling the flowers with a dark olive-brown, powdery mass of spores. Infection systemic. Distal, sterile flowers of an infected inflorescence develop anthers producing spores. Spores variable in shape and size, globose, subglobose, ovoid, curved, pyriform, tear-shaped, or irregularly elongate, 5.5-9.5 × 6.5-12(-14) $\mu \mathrm{m}$, olive-brown; wall even or slightly uneven, c. $0.5 \mu \mathrm{m}$ thick, finely, densely verruculose, spore profile finely wavy to irregularly verruculose-tuberculate, tubercles often irregularly fused. Spore germination results in 4-celled basidia, usually the uppermost three-celled part separating from the basal cell, producing laterally 
and terminally elongate, sessile, budding basidiospores, or basidiospores develop on short sterigmata.

\section{Anthracoidea Bref.}

\section{Key to the Anthracoidea species based on host plant taxonomy}

1. On Carex subgen. Vignaea, sect. Stenophylla, Carex stenophylla. A. eleocharidis

- On Carex subgen. Carex 2

2. On sect. Acrocystis (= Montanae), Carex tomentosa.

A. tomentosae

- Not on sect. Acrocystis . .3

3. On sect. Glaucae, Carex flacca ssp. serrulata A. pratensis

- Not on sect. Glaucae .4

4. On sect. Lamprochlaenae, Carex liparocarpos.. A. caryophylleae

- Not on sect. Lamprochlaenae 5

5. On sect. Paludosae, Carex songorica A. songorica

- Not on sect. Paludosae .6

6. On sect. Rhomboidales, Carex michelii. A. michelii

- Not on sect. Rhomboidales 7

7. On sect. Spirostachyae, Carex diluta A. caricetorum

- Not on sect. Spirostachyae .8

8. On sect. Carex s.lat., Carex melanostachya A. melanostachyae

- On various sections, Carex sp. caricis s. lat.
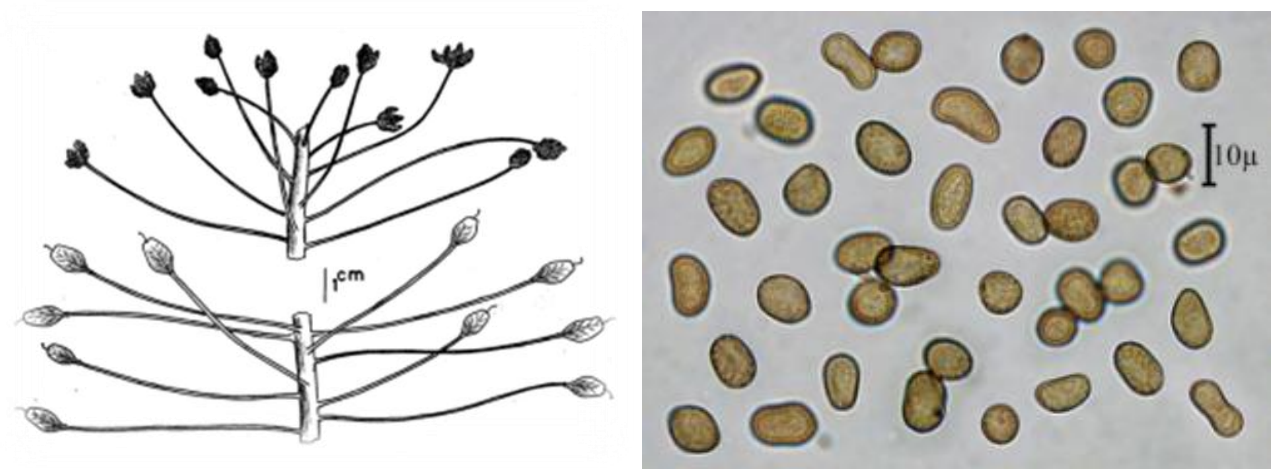

Fig.1 - Antherospora tourneuxii on Bellevalia saviczii (14782), infected plant (left) and teliospores (right)

On Hyacinthaceae (Liliaceae s. 1.):

Muscari sp., Hormozgan Prov., Minab.

3. Anthracoidea caricetorum (Speg.) Nannf., Symb. Bot. Upsal. 22: 21, 1979.

Cintractia caricetorum Speg., 1925.

Sori in ovaries, black, compact. Spores small-sized, flattened, in side view elliptic, 8-11 $\mu \mathrm{m}$ wide, in plane view circular, elliptic to subangularly irregular, $12-16 \times 12-17.5 \mu \mathrm{m}$, yellowish brown; wall even, $0.5-1 \mu \mathrm{m}$ thick, no internal swellings, no protuberances and light-refractive areas, surface finely verruculose, the spore profile smooth to finely wavy, in SEM moderately dense and low verruculose.

On Cyperaceae: Carex (subgen. Carex, sect. Spirostachyae), C. diluta M. Bieb., Semnan Prov., Nezva Mt. area, near Orim village, 2100 m, 5.VII.1959, leg. P. Wendelbo, O.

Jørstad (1960:27) studied this collection (under the name of Cintractia caricis) and wrote: "Spores 17-20 $\mu$ in diam., more or less angulate, wall c. $1.5 \mu$ thick, densely and finely verrucose." 
The type of A. caricetorum is on Carex fuscula d'Urv. (subgen. Carex; sect. Spirostachyae), Argentina, Patagonia, Santa Cruz, Rio Santa Cruz, II.1882, C. Spegazzini. Additional host plant is C. fuscula var. distenta (Kunze) Kük. (Chile; Nannfeldt 1979:21).

4. Anthracoidea caricis (Pers.) Bref., Unters. Gesammtgeb. Mykol. 12: 144, 1895, s. lat. Uredo caricis Pers. 1801. - Caeoma caricis (Pers.) Link, in Willdenow 1825. - Ustilago caricis (Pers.) Unger 1836. - Cintractia caricis (Pers.) Magnus 1896a('1895').

Uredo urceolorum DC. 1815 (nom. nov. superfl. pro Uredo caricis Pers.).

Cintractia caricis var. intermedia Savile 1952.

Sori in ovaries, scattered in the inflorescence, forming globose, black, hard bodies, 1-2 mm in diam., initially covered by a thin, greyish membrane, later black, powdery on the surface. Spores flattened, 11-15 $\mu \mathrm{m}$ wide, in plane view subcircular, angular or irregular, $14-20(-22) \times 15-25(-28) \mu \mathrm{m}$, reddish brown; wall of uneven thickness, 1-3(-4) $\mu \mathrm{m}$, thickest at the angles, 1-3 indistinct internal swellings may be present, light-refractive areas uncommon or lacking, surface conspicuously verrucose, spore profile wavy or finely serrulate, in SEM covered with low, rounded warts and minute, dense papillae between warts. Spore germination of Anthracoidea-type.

On Cyperaceae: Carex sp., Tehran Prov., Karaj-Chalus road, Asara village.

5. Anthracoidea caryophylleae Kukkonen, Ann. Bot. Soc. Zool.-Bot. Fenn. 'Vanamo' 34(3): 53, 1963.

Sori in some ovaries of an inflorescence forming globose, black, hard bodies, 1-2 $\mathrm{mm}$ in diam., when young covered by a thin, greyish membrane, later powdery on the surface, partly hidden by the utricles. Spores slightly flattened, in side view 11-13 $\mu \mathrm{m}$ wide, in plane view ovate, subpolygonal to irregular, 15-20 × 16-24(-27) $\mu \mathrm{m}$, dark reddish brown; wall 1-3 $\mu \mathrm{m}$ thick, thickest at the angles, usually with 1-3 internal swellings and sometimes with light-refractive spots in the thickest areas, surface nearly smooth, sparsely punctate to finely verruculose, warts not fusing, spore profile appearing smooth or nearly so, in SEM sparsely to densely verruculose with rounded, low warts, between the warts minutely, densely verruculose. Spore germination of Anthracoidea-type (Fig. 2).

On Cyperaceae: Carex (subgen. Carex, sect. Lamprochlaenae), C. liparocarpos Gaud., East Azarbaijan Prov., Kaleibar, Ghaleh Babak ruin, IRAN 14047 F; East Azarbaijan Prov., Arasbaran protected area, Kaleibar city toward Ghaleh Babak ruin, IRAN 13989 F; East Azarbaijan Prov., Arasbaran protected area, Makidi valley, IRAN 11775 F.

6. Anthracoidea eleocharidis Kukkonen, Trans. Brit. Mycol. Soc. 47: 244, 1964.

Anthracoidea duriusculae L. Guo 2006a.

Sori in some ovaries of an inflorescence forming globose, black, hard bodies, 2-3 $\mathrm{mm}$ in diam., when young covered by a silvery membrane that flakes away exposing the black, agglutinated spore mass with a powdery surface. Spores moderately flattened, in plane view circular, ovoid or slightly irregular, 11-15(-17) × 13-20(-21) $\mu \mathrm{m}$, medium reddish brown; wall even, 0.7-1.5 $\mu \mathrm{m}$ thick, without internal swellings, surface distinctly papillate, spore profile from almost smooth or very finely wavy to finely wavy or serrulate, in SEM papillae $0.1-0.5 \mu \mathrm{m}$ high, with a rounded tip, forming short rows or groups, rarely fusing; wall surface between the papillae smooth. Spore germination of Anthracoidea-type (Fig. 3).

On Cyperaceae: Carex (subgen. Vignaea, sect. Stenophylla), C. stenophylla Wahlenb. (C. pachystylis J. Gay), Fars Prov., Fasa Forest, IRAN 13288 F; Fars Prov., Old road of Shiraz city to Esfahan city, after Sivand village, IRAN 13286 F; Fars Prov., Old road of Shiraz to Esfahan city, near Naghsh-e Rajab, historical sculpture, Rahmat Mountain, IRAN 13287 F; Tehran Prov., Khojir National Park, IRAN 2000 F; East Azarbaijan Prov., Mianeh city to Zanjan city, IRAN 8073 F; East Azarbaijan Prov., Mianeh city, at the Pole-Dokhtar historical bridge, IRAN 8072 F; West Azarbaijan Prov., Boundry of Iran \& Turkey, Bazargan road to Cheshmeh Soraya, Yarom Ghiyah village, IRAN 8070 F; Zanjan Prov., Zanjan to Hamadan road, 75 Km N. Hamadan city, Ghariech village, Chahar 
Boulagh Mt., IRAN 7965 F; Fars Prov., Sarvestan city, IRAN 8007 F; Kermanshah Prov., Nojivaran, N slope of Paru Mt., IRAN 14784 F.

On Carex sp., West Azarbaijan Prov., Salmas to Oroumieh, Ghushchi Pass, IRAN 8759 F; Mazandaran Prov., Karaj-Chalus road, IRAN 8235 F; Golestan Prov., Gorgan city, Golestan National Park, Sulgerd area, IRAN 8330 F; Kordestan Prov., Palangan village, IRAN 8297 F.

7. Anthracoidea melanostachyae Denchev \& T. Denchev, Mycol. Balcan. 8: 153-155, 2011.

Sori in some ovaries, scattered in the inflorescence, forming black, hard, ovoid, 2-3 mm long bodies, dusty on the surface, when young covered by a white, thin fungal layer. Spores slightly flattened, in side view elliptic, 9.5-12 $\mu \mathrm{m}$ wide, in plane view from subcircular to usually irregular, $12-17.5 \times$ 14-20(-22) $\mu \mathrm{m}$, reddish brown; wall uneven, 0.8-3 $\mu \mathrm{m}$ thick, thickest at the angles and protuberances, some weak internal swellings and light-refractive spots may be present, surface evidently verruculose, spore profile smooth to wavy.

Spore germination unknown (Fig. 4).

On Cyperaceae: Carex (subgen. Carex, sect. Carex s. lat.), C. melanostachya M. Bieb. ex Willd., East Azarbaijan Prov., Kalibar to Khodaafarin, Govar village, IRAN 11967 F; Tehran Prov., Firuz-Kuh city to Ghaemshahr city, Gaduk, IRAN 11362 F; Golestan Prov., Gorgan city, Golestan National Park, Tange Gol, Sar-Cheshmeh, alt. 670-730 m, 22.VII.1991, M. Abbasi, IRAN 83233 F, HUV 20094; Golestan Prov., Azadshahr city IRAN 11645 F.

8. Anthracoidea michelii Vánky, Bot. Not. 132: 223, 1979.

Sori in ovaries, scattered in the inflorescence, forming subglobose, hard, black bodies, 2-3 $\mathrm{mm}$ in diam., partly hidden by the glumes and utricles. Spores moderately flattened, in side view 10-13 $\mu \mathrm{m}$ wide, in plane view variable in form, subangular to irregular, 13-20 × 14-24(-30) $\mu \mathrm{m}$, medium to dark reddish brown; wall unevenly $1-3(-5) \mu \mathrm{m}$ thick, thickest at the angles and protuberances, lightrefractive areas common, 1-2 internal swellings as well as protuberances sometimes present, surface distinctly verruculose, spore profile papillate to serrulate, in SEM with moderately densely, somewhat irregularly dispersed, rounded warts (Fig. 5).

On Cyperaceae: Carex (subgen. Carex, sect. Rhomboidales), C. michelii Host, East Azarbaijan Prov., Kalaibar city, Makidi valley, IRAN 11966 F.

9. Anthracoidea pratensis (Syd.) Boidol \& Poelt, Ber. Bayer. Bot. Ges. 36: 23, 1963.

Cintractia pratensis Syd. 1924.

Sori in ovaries, scattered in the inflorescence, forming subglobose to ovoid, black, hard bodies, 2-3 $\mathrm{mm}$ in diam., powdery on the surface. Spores flattened, in side view 13-15 $\mu \mathrm{m}$ wide, in plane view subcircular, broadly elliptic, subangular to irregular, often with protuberances, 17-27 × 19-30 $\mu \mathrm{m}$, dark reddish brown; wall uneven, $1-4(-6) \mu \mathrm{m}$ thick, thickest at the angles and protuberances, internal swellings low, 1-2(-3), only rarely seen, light-refractive areas often present, surface verruculose, the warts often forming short rows and sometimes partly confluent, spore profile almost smooth to finely serrulate, in SEM with low, rounded, often confluent warts. Spore germination of Proceres-type (Fig. 6).

On Cyperaceae: Carex (subgen. Carex, sect. Glaucae), C. flacca Schreb. ssp. serrulata (Biv.) Malag., Golestan Prov., Gorgan city, Golestan National Park, Tange gol to Sar-Cheshmeh, IRAN 11539 F; Golestan Prov., Fazelabad city, Alestan village, IRAN 13928 F.

10. Anthracoidea songorica Vánky, in Vánky \& Abbasi, Rostaniha 12(2): 182, 2012a.

Sori forming black, globose or ovoid, hard bodies in some ovaries in the inflorescence, powdery on the surface, 1.5-2.5 mm in diameter. Spores globoid, broadly ellipsoidal, subpolyhedrally irregular, occasionally elongated, with a subacute or acute tip, 13.5-22.5 × 19-27(-30) $\mu \mathrm{m}$, dark reddish brown; wall uneven, 1-3.5(-5.5) $\mu \mathrm{m}$ thick, thickest at the angles, no internal swellings, light refracting spots occasionally present, surface densely, prominently verrucose, spore profile wavy to finely serrulate. Spore germination unknown (Fig. 7). 
On Cyperaceae: Carex (subgen. Carex, sect. Paludosae), C. songorica Kar. \& Kir., Golestan Prov., Gorgan city, Golestan National Park, alt. 1100 m, 22.VII.1991, leg. M. Abbasi, HUV 21968, IRAN $11642 \mathrm{~F}$.

11. Anthracoidea tomentosae Vánky, Bot. Not. 132: 227, 1979.

Sori in ovaries, scattered in the inflorescence, forming hard, subglobose bodies, 1.5-2 $\mathrm{mm}$ in diam., when young covered by a silvery membrane that soon flakes away to reveal the agglutinated, black spore mass, partly hidden by the utricles. Spores slightly flattened, in side view 13-16 $\mu \mathrm{m}$ wide, in plane view subangular to irregular, (12-)13.5-23 $\times(18-) 20-28 \mu \mathrm{m}$, reddish brown; wall moderately uneven, 1.5-3(-4) $\mu \mathrm{m}$ thick, thickest at the angles and protuberances, rarely with light-refractive spots and 1-3 low internal swellings, surface finely, densely punctate, spore profile finely serrulate, in SEM with sparsely to dense, often confluent, rounded warts, the spaces between the warts extremely finely and densely verruculose. Spore germination of Proceres-type (Fig. 8).

On Cyperaceae: Carex (subgen. Carex, sect. Acrocystis = Montanae), C. tomentosa L., East Azarbaijan Prov., Arasbaran protected area, Khodaafarin village, Vaighan forked road, IRAN 11710 F.

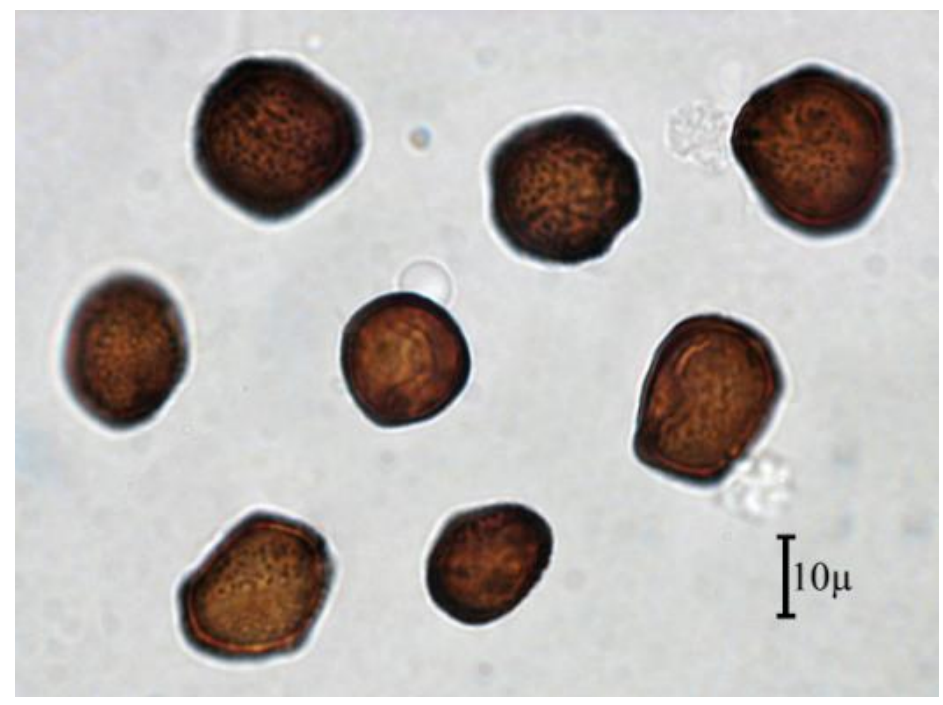

Fig.2 - Anthracoidea caryophylleae on Carex liparocarpos (14047)

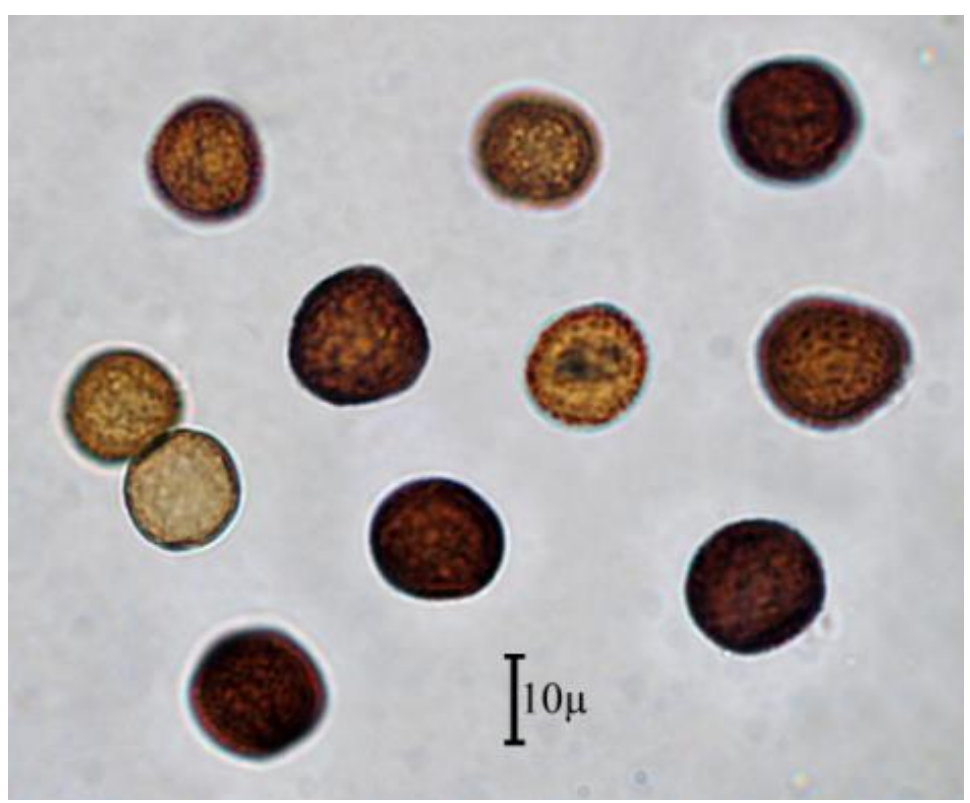

Fig.3 - Anthracoidea eleocharidis on Carex pachystylis (13286) 


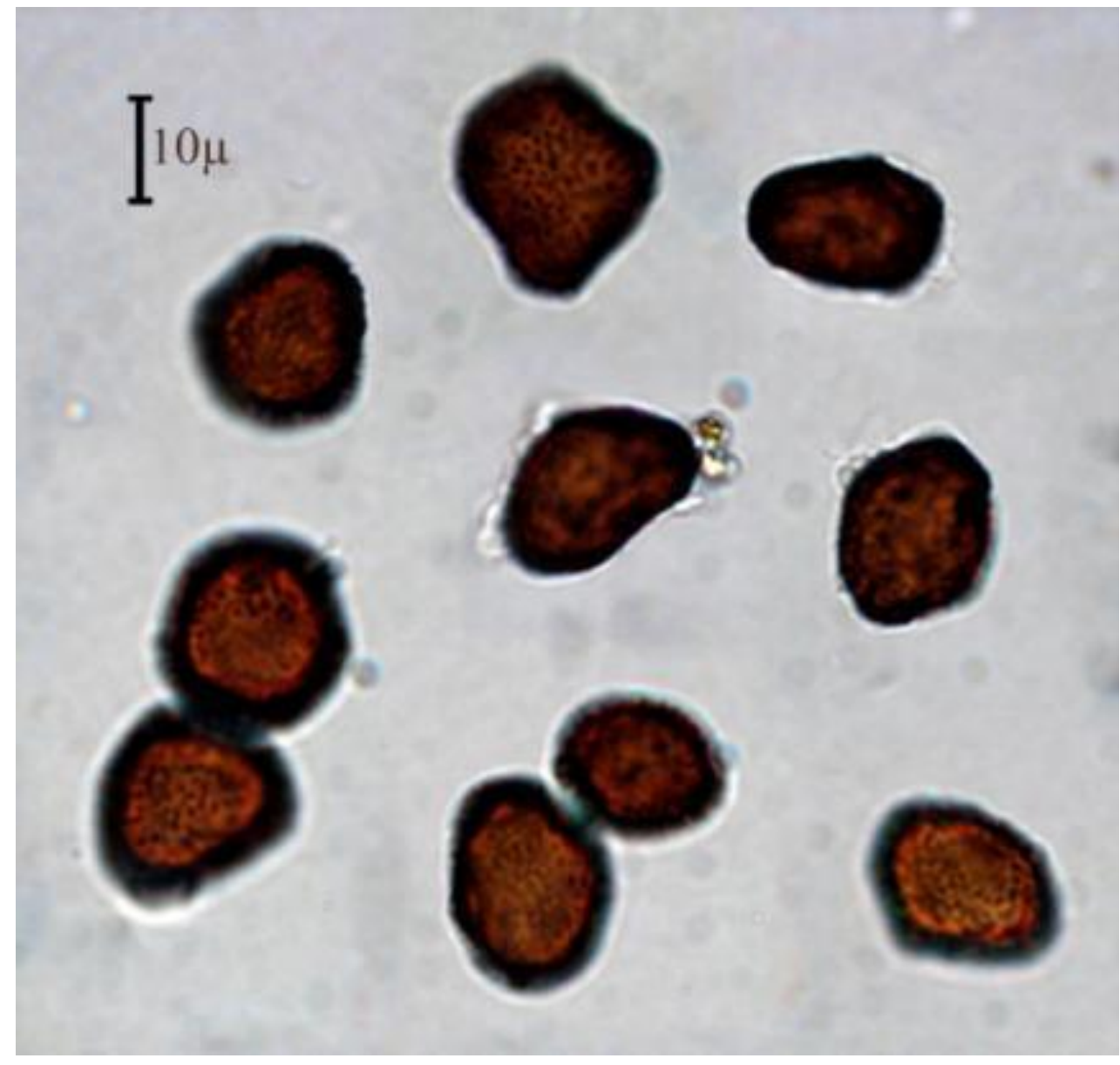

Fig.4 - Anthracoidea melanostachyae on Carex melanostachya (11362)

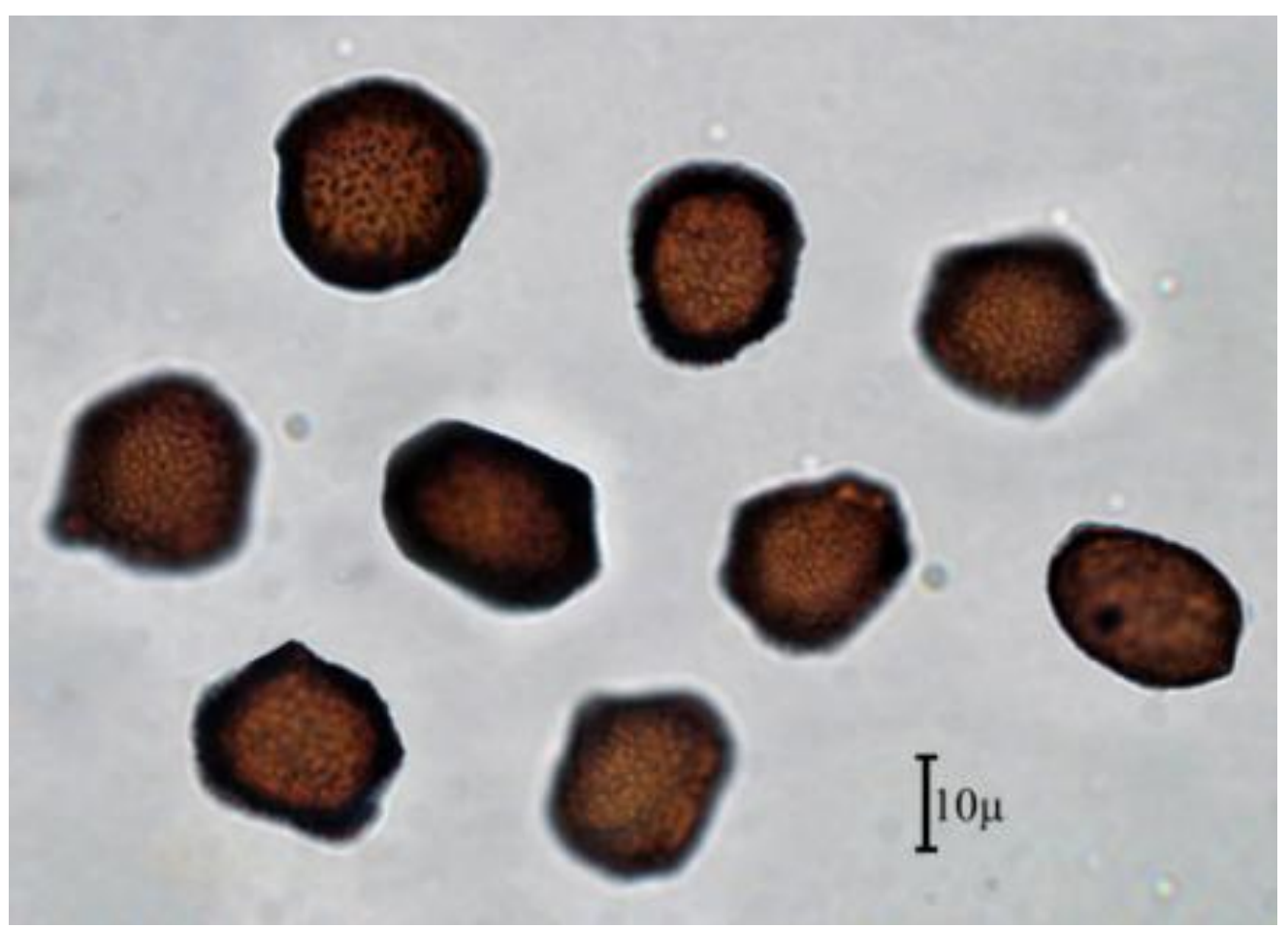

Fig.5 - Anthracoidea michelii on Carex michelii (11966) 


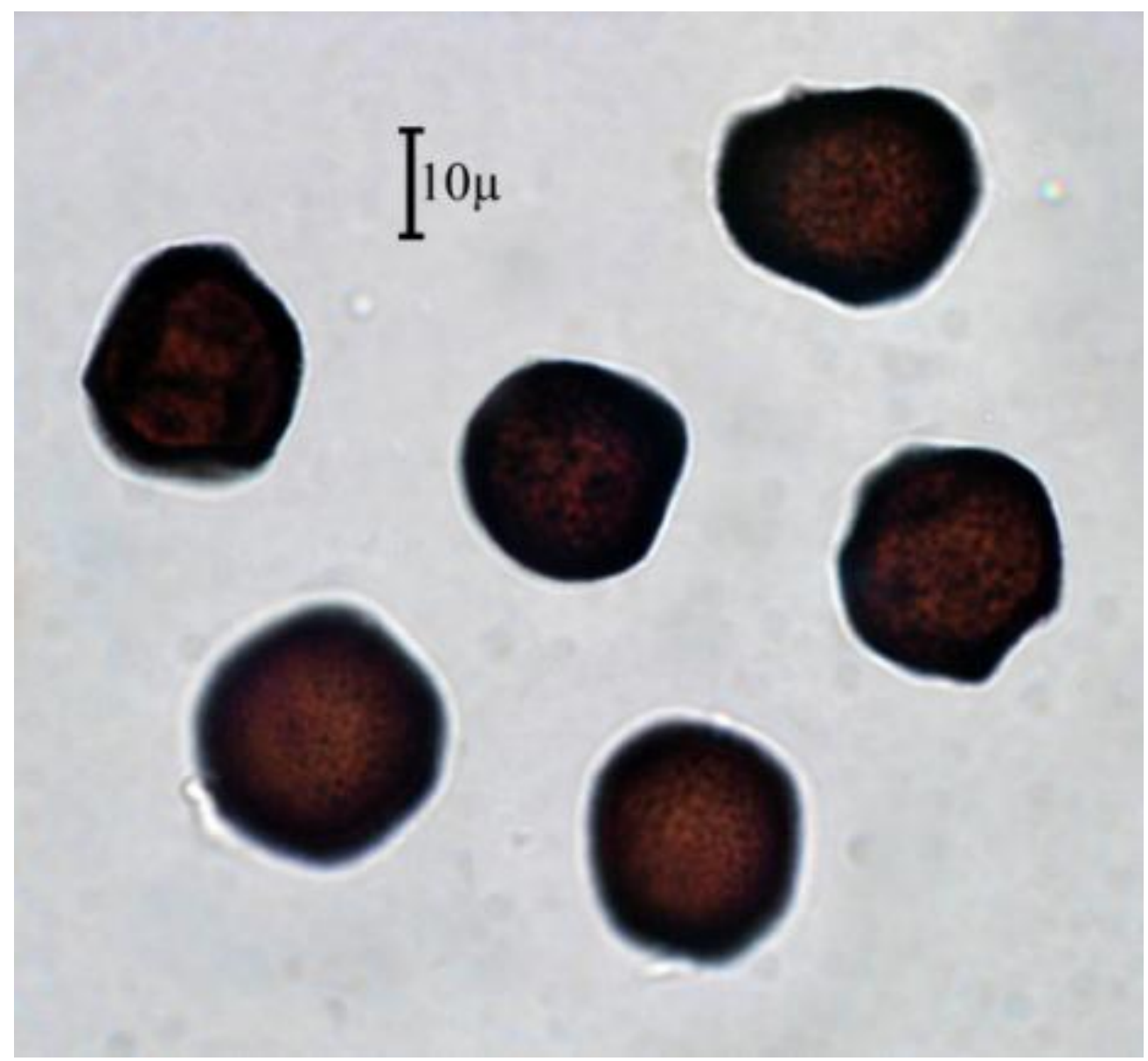

Fig.6 - Anthracoidea pratensis on Carex flacca ssp. serrulata (11539)

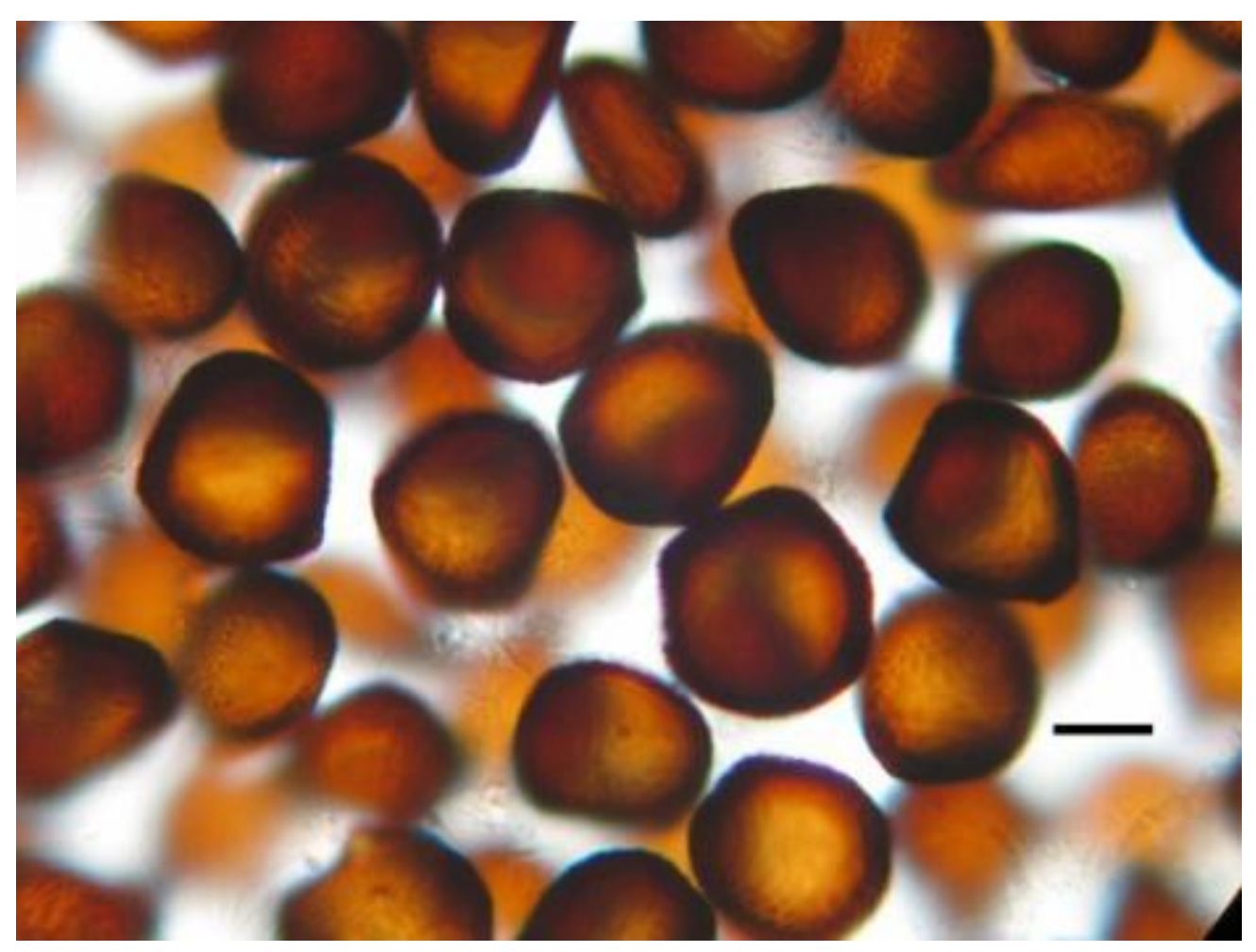

Fig.7 - Anthracoidea songorica on Carex songorica (11642), Bar=10 $\mu \mathrm{m}$ 


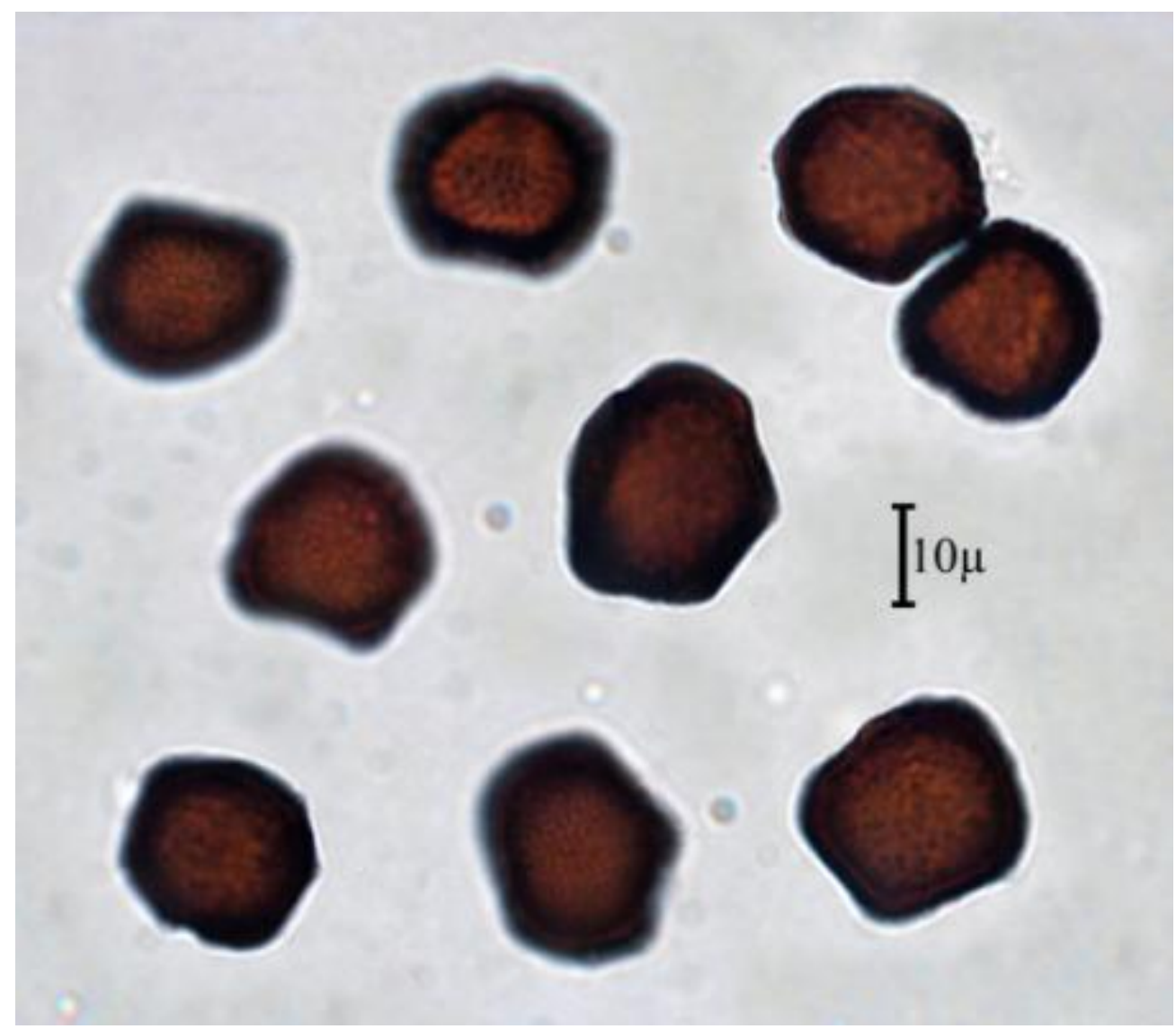

Fig.8 - Anthracoidea tomentosae on Carex tomentosa (11710)

\section{Entyloma de Bary}

\section{Key to the Entyloma species based on host plant taxonomy}

1. On Asteraceae

- Not on Asteraceae

2. On Rhagadiolus

E. rhagadioli

- On Tragopogon

E. tragopogonis

3. On Boraginaceae (Myosotis).

E. fergussonii

- Not on Boraginaceae

4. On Geraniaceae (Geranium).

E. atlanticum

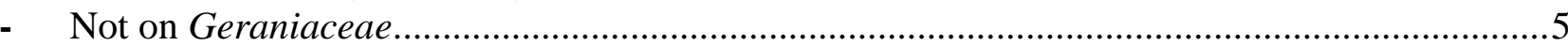

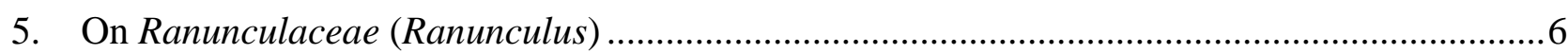

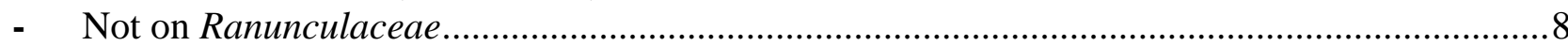

6. Sori as pustules or swellings; spore wall uneven, 1.5-9.5 $\mu$ m thick ..................E. microsporum

- Sori flat or slightly bullate; spore wall even or uneven, 1-7 $\mu \mathrm{m}$ thick

7. Sori slightly bullate; spore wall uneven, $2.5-7 \mu \mathrm{m}$ thick . .7

- $\quad$ Sori flat; spore wall even, 1-2 $\mu$ m thick E. majewskii

8. On Scrophulariaceae (Linaria) E. linariae

- On Solanaceae (Physalis)..... E. australe

12. Entyloma atlanticum Massenot, in Guyot, Malençon \& Massenot, Rev. Pathol. Vég. Entomol. Agric. France 37: 187, 1958.

Entyloma geranii M.N. Kuznetzova \& Schwarzman, in Schwarzman 1960.

Sori in leaves as slightly convex, yellowish white to violet tinted brown spots, $2-3 \times 2-5 \mathrm{~mm}$ in diam. Spores densely agglutinated, variable in shape and size, occasionally subglobose, usually 
subpolyhedrally irregular, $10-17 \times 13-22 \mu \mathrm{m}$, subhyaline to pale yellow; wall $1-4.5 \mu \mathrm{m}$ thick, twolayered, inner layer even, c. $0.5 \mu \mathrm{m}$ thick, outer layer uneven, 0.5-3(-4) $\mu \mathrm{m}$ thick, smooth. Anamorph may be present (Fig. 9).

On Geraniaceae: Geranium tuberosum L., Tehran Prov., Hraz road, 60 km E of Tehran city, Elburz Mts., "Emamzadeh-Hashem", IRAN 7229 F; Esfahan Prov., Golpayegan, 140 km NW of Esfahan city, Golestan Kuh Mt., IRAN 7230 F; Ilam Prov., Ilam city, Renow park, IRAN 6429 F.

13. Entyloma australe Speg., Anales Soc. Ci. Argent. 10: 5, 1880.

Protomyces physalidis Kalchbr. \& Cooke 1880.

- Entyloma physalidis (Kalchbr. \& Cooke) G. Winter 1883.

Entyloma besseyi Farl. 1883.

Sori in leaves forming circular to angular spots, at first light yellow, later brown, 1-5 mm in diam. or larger when confluent. Spores globose, subglobose, ellipsoidal to somewhat polyhedral or irregular, $8-13 \times 9-16 \mu \mathrm{m}$, hyaline or with a pale yellowish brown tint; wall even or uneven, $1-2.5(-3) \mu \mathrm{m}$ thick, smooth. Anamorph present in whitish tufts within the spots; conidia linear, often curved, 1.5$2.5 \times 24-50 \mu \mathrm{m}$.

On Solanaceae: Physalis alkekengi L., Mazandaran Prov., Ghaemshahr, Gharakhil Agricultural Station, IRAN 6428 F.

14. Entyloma fergussonii (Berk. \& Broome) Plowr., Monogr. Brit. Ured. and Ust.: 289, 1889. Protomyces fergussonii Berk. \& Broome 1875.

Entyloma canescens J. Schröt. 1877.

Sori in leaves as circular, amphigenous, scattered spots, initially yellowish white, indefinite, later brown, 0.5-3(-5) $\mathrm{mm}$ in diam. Spores globose, ovoid to slightly irregular, 8-13 $\times 9-14(-16) \mu \mathrm{m}$, subhyaline to pale yellowish brown; wall smooth, even, 0.5-1.5 $\mu \mathrm{m}$ thick. Anamorph (Entylomella myosotidis (Sacc.) Höhn.) present.

On Boraginaceae: Myosotis sylvatica Hoffm., Mazandaran Prov., Chalous-road, Siah bisheh village.

15. Entyloma majewskii Vánky \& M. Lutz, Polish Bot. J. 55: 276, 2010.

Sori on leaves as slightly bullate, circular or broadly elliptic spots, $1-1.5(-2) \mathrm{mm}$ in diameter or larger by confluence, first white, later whitish-green or pale yellowish-brown, evident on both sides of the leaves. Spores crowded, globose, subglobose, ellipsoidal to slightly irregular, with one, rarely two flattened sides, $10.5-16(-17.5) \times 10.5-17(-18.5) \mu \mathrm{m}$, subhyaline to pale yellowish tinted; wall even to usually unevenly thickened, two-layered, $2.5-7 \mu \mathrm{m}$ wide, including the even, $0.8-1 \mu \mathrm{m}$ thick endospore, spore surface smooth. Anamorph absent (Fig. 10).

On Ranunculaceae: Ranunculus ficaria L. (Ficaria ranunculoides Roth.). Tehran Prov., 60 km E of Tehran, Haraz road, Elburz Mts., "Emamzadeh-Hashem", alt. 2610 m, HUV 14888, IRAN 7272 F; Golestan Prov., 35 km SW of Gorgan city, alt. 2250 m, HUV 14887, IRAN 7273 F; Kuhgiluyeh va Buyrahmad Prov., Savers Mt., IRAN 8071 F.

Remarks - Entyloma ficariae mentioned by Vánky \& Ershad (1993: 2), and by Ershad (2001: 48), on Ficaria ranunculoides from Iran, represents E. majewskii. The two species differ in their morphological and molecular phylogenetic characters. In E. ficariae the sori are flat, circular or angular, evident on both sides but more expressed on the abaxial side of the leaves. The spores in $E$. ficariae are loosely crowded or scattered in the host tissue, globose, subglobose, ovoid to broadly ellipsoidal, sometimes slightly irregular, 10-14.5(-15) × 11-16 $\mu \mathrm{m}$, with an evenly $1-2.5(-3) \mu \mathrm{m}$ thick, two-layered wall. Anamorph in E. majewskii absent, in E. ficariae usually present.

16. Entyloma linariae J. Schröt., in Cohn, Beitr. Biol. Pfl. 2: 371, 1877.

Sori in leaves as round, flat, amphigenous spots, 0.5-2(-3) $\mathrm{mm}$ in diam., initially white, later pale brown, with age the middle often becomes perforated. Spores globose, subglobose or sometimes 
slightly irregular, 9.5-13 $\times 10-14 \mu \mathrm{m}$, subhyaline to pale yellow; wall two-layered, even, c. $1 \mu \mathrm{m}$ thick, smooth. Anamorph present.

On Scrophulariaceae: Linaria sp., Golestan Prov., Shahvar Mt.

17. Entyloma microsporum (Unger) J. Schröt., in Rabenh., Fgi eur. no. 1872, 1874.

Protomyces microsporus Unger 1833. - Entyloma ungerianum de Bary 1874 (nom. nov. superfl. pro. P. microsporus).

Caeoma bullosum Sacc. 1915.

Entyloma microsporum (Unger) J. Schröt. var. pygmaeum Allesch., in Allesch. \& Henn. 1897. Entyloma pygmaeum (Allesch.) Cif. 1928.

Sori in leaves and petioles as hard pustules or swellings, at first yellowish white, disk-like, later brown, swollen, subglobose or fusiform, wart-like, with cracked surface. Spores embedded in the host tissue, crowded, globose, ovoid or irregular, 10-16 × 11-23 $\mu \mathrm{m}$, light yellow; wall two-layered, inner layer yellow, uniformly c. 0.5-1 $\mu$ m thick, outer layer hyaline, uneven, 1-9 $\mu \mathrm{m}$ thick, smooth. Spore germination results in aseptate basidium developing an apical whorl of 4-8 simultaneously produced basidiospores, which fuse in pairs giving rise to septate and ramified hyphae and/or to secondary ballistospores. Anamorph absent (Fig. 11).

On Ranunculaceae: Ranunculus muricatus L., Golestan Prov., Naharkhoran area, $10 \mathrm{~km} \mathrm{~S}$ of Gorgan city, IRAN $7271 \mathrm{~F}$.

18. Entyloma ranunculi-repentis Sternon, L'hétérogenéité du genre Ramularia, These, Nancy: 34 $\& 45,1925$.

Entyloma wroblewskii Kochman 1934.

Entyloma ranunculi-scelerati Kochman 1936.

Entyloma ranunculacearum Kochman 1936.

Entyloma ranunculorum Liro 1939

Similar to Entyloma ficariae (with which it was merged by several authors) but has smaller spores $(8-13 \times 9-14 \mu \mathrm{m})$, thinner spore wall $(1-2 \mu \mathrm{m})$ and usually fewer, more dispersed spores in the sori. Anamorph present.

On Ranunculaceae: Ranunculus sceleratus L., Gilan Prov., Bandar-Anzali, Anzali lagoon, IRAN $7270 \mathrm{~F}$.

19. Entyloma rhagadioli Pass., Nuovo Giorn. Bot. Ital. 9: 239, 1877.

Sori in leaves as orbicular, amphigenous spots, 1-3 mm wide, initially whitish, later reddish brown or brown. Spores often agglutinated in irregular groups, variable in shape and size, globose, ovoid, irregular, 8-12 × 9-16 $\mu \mathrm{m}$, pale yellowish brown; wall uneven, (1-)2-3(-4) $\mu \mathrm{m}$ thick, smooth. Anamorph present (Fig. 12).

On Asteraceae: Rhagadiolus stellatus Gaertn., Kohgiluyeh va Buyerahmad Prov., Dehdasht city, Taleh Mt., IRAN 8034 F.

20. Entyloma tragopogonis Lagerh., Bull. Soc. Mycol. France 15: 100, 1899 (as 'tragopogi'). Entyloma tragopogonis Wróbl. 1914.

Sori in leaves as flat, amphigenous, ovoid to elongate spots, initially yellow, later pale brown, 1-5 $\mathrm{mm}$ long or larger by confluence. Spores globose, subglobose, ovoid, subpolyhedral to irregular, 8 $12 \times 10-14 \mu \mathrm{m}$, pale yellow to pale yellowish brown, densely crowded; wall smooth, two-layered, even to slightly uneven, $1.5-2.5(-3) \mu \mathrm{m}$ thick.

On Asteraceae: Tragopogon graminifolius DC., East Azarbaijan Prov., suburb of Tabriz city. 


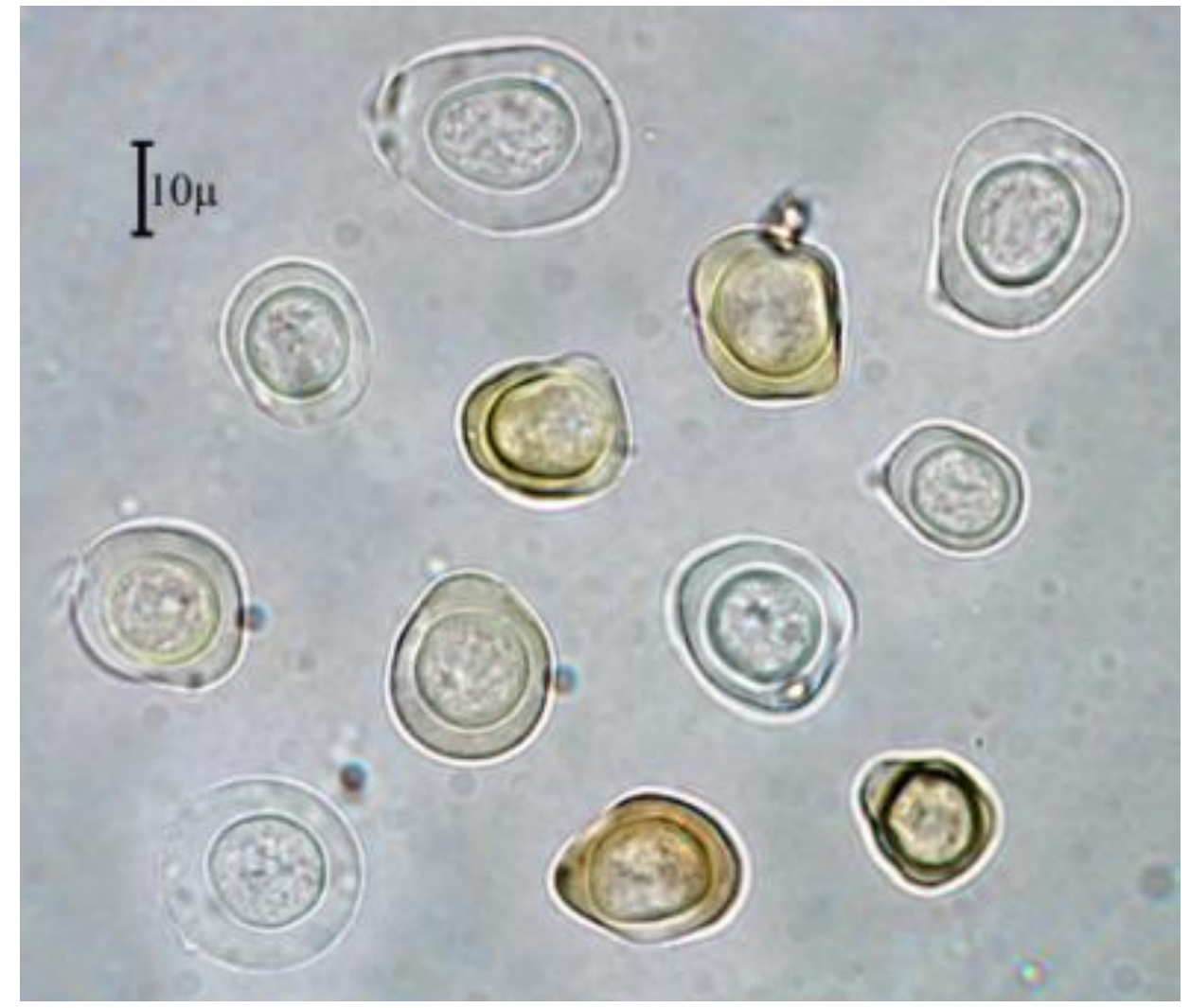

Fig.9 - Entyloma atlanticum on Geranium tuberosum (7230)

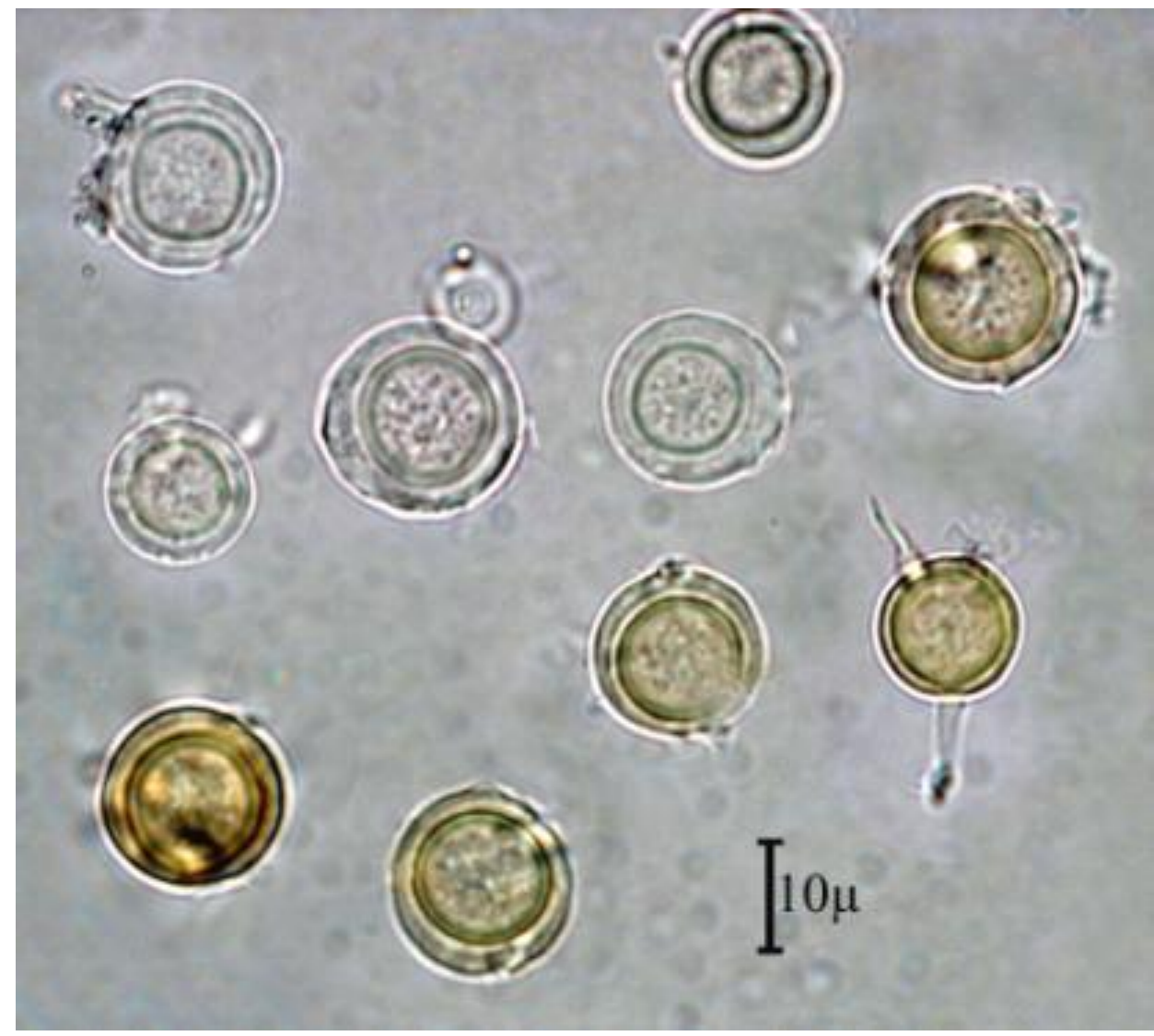

Fig.10 - Entyloma majewskii on Ranunculus ficaria (8071) 


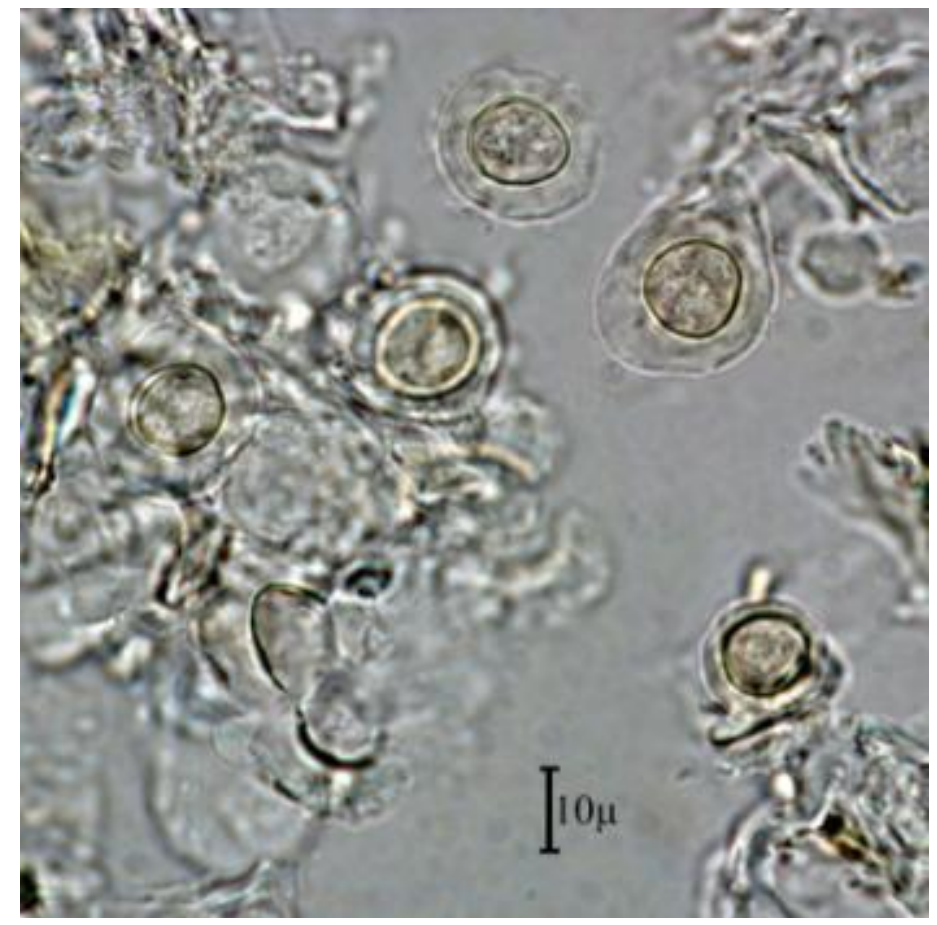

Fig.11 - Entyloma microsporum on Ranunculus muricatus (7271)

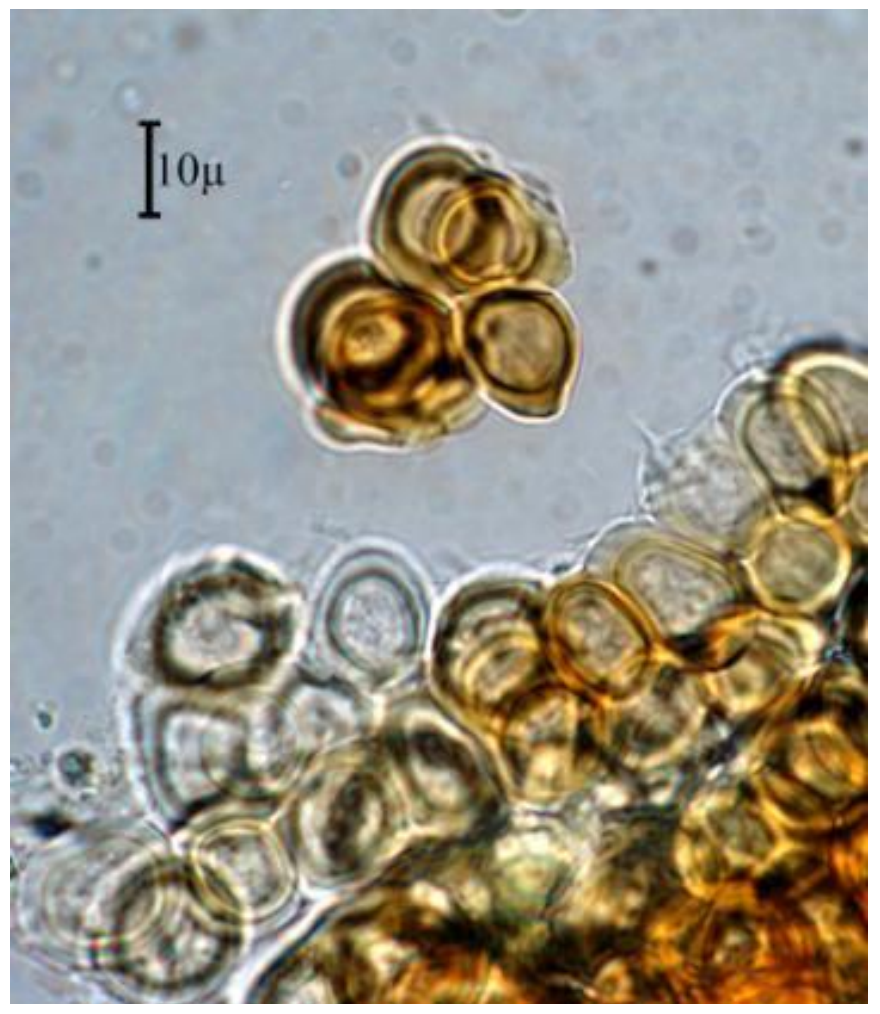

Fig.12 - Entyloma rhagadioli on Rhagadiolus stellatus (8034)

\section{Farysia Racib.}

21. Farysia thuemenii (A.A. Fisch. Waldh.) Nannf., in Lindeberg, Symb. Bot. Upsal. 16(2): 51, 1959.

Ustilago thuemenii A.A. Fisch. Waldh. 1878. 
Uredo segetum var. caricis DC. 1805. - Uredo olivacea DC. 1815. - Caeoma olivaceum (DC.) Schltdl. 1824. - Erysibe olivacea (DC.) Wallr. 1833. - Ustilago olivacea (DC.) Tul. \& C. Tul. 1847. - Elateromyces olivaceus (DC.) Bubák 1912. - Farysia olivacea (DC.) Syd. \& P. Syd. 1919 (later homonym). - Farysia caricis (DC.) Liro 1938.

Cintractia caricicola Henn. 1895.

Ustilago caricicola Tracy \& Earle 1899.

Ustilago cariciphila Speg. 1925. - Cintractia cariciphila (Speg.) Cif. 1931.

Cintractia caricis (Pers.) Magnus f. minor Sousa da Camara \& Oliveira 1945.

Sori in scattered flowers arising from the floral pedicels, initially as ovoid to cylindrical bodies, concealed by the utricle (perigynium), later protruding, covered by a greyish white to light brown fungal peridium that becomes shredded and exposes the olivaceous brown, dusty spore mass traversed by numerous, prominent fascicles of hyphae (elaters), commonly extending beyond the sori. Spores very variable in shape and size, globose, subglobose (3-7 $\mu \mathrm{m}$ in diam.), ovoid, elongate (3-12 $\mu \mathrm{m}$ long), often with truncate ends, irregular, curved, pyriform, or subtriangular, pale olivaceous brown, the surface finely but distinctly verruculose, in SEM the warts truncate or rounded, very dense, sometimes confluent. Spore germination results in 4-celled basidium (of $3+$ 1 type, one cell remaining in the spore) budding off several ovoid basidiospores. Occasionally a long hypha is borne which was not seen to form basidiospores (Fig. 13).

On Cyperaceae: Carex riparia Curt., Gilan Prov., 10 km SE of Rasht city, IRAN 7263 F; Gilan prov., Langerud, Chamkhaleh, Talab-e Amir kelayeh, IRAN 8425 F.

\section{Jamesdicksonia Thirum., Pavgi \& Payak}

22. Jamesdicksonia dactylidis (Pass.) R. Bauer, Begerow, A. Nagler \& Oberw., Mycol. Res. 105: 422, 2001, s. lat.

Thecaphora dactylidis Pass., in A.A. Fisch. Waldh. 1877a('1876'). - Entyloma dactylidis (Pass.) Cif. 1924. - Melanotaenium dactylidis (Pass.) Denchev 1995.

Entyloma crastophilum Sacc. 1879.

Entyloma crepinianum Sacc. \& Roum., in Roum. \& Sacc. 1881.

Entyloma catabrosae Johanson 1884.

Entyloma camusianum Har. 1896.

Entyloma schweinfurthii Henn. 1902.

Entyloma hieroënse Har. \& Pat. 1904.

Entyloma phalaridis Speg. 1912. - Ustilago phalaridis (Speg.) Cif. 1931.

Entyloma cynosuri Gonz. Frag. \& Cif., in Cif. 1925.

Tuburcinia castellana Gonz. Frag. 1926. - Urocystis castellana (Gonz. Frag.) Zundel 1953.

Entyloma korshinskyi Lavrov 1937.

Entyloma camusianum Har. var. pratense Lavrov 1937.

Entyloma polypogonis Vienn.-Bourg. 1937.

Entyloma alopecurivorum Lavrov 1938.

Entyloma holci Liro 1939.

Entyloma deschampsiae Liro 1939.

Entyloma lagerheimianum Liro 1939.

Entyloma nubilum Liro 1939.

Entyloma espinosae Unamuno 1940.

Tolyposporella semenoviana Lavrov 1934. - Entyloma semenoviana (Lavrov) Gutner 1941. Ustilago semenoviana (Lavrov) Cif. 1959.

Entyloma gaudinae Vienn.-Bourg. 1954,'1953' (nom. inval.)

Entyloma anadelphiae Vienn.-Bourg. 1957.

Entyloma vulpiae Massenot, in Guyot, Malençon \& Massenot 1960.

Sori in leaves and sheaths as short, linear to ovate, lead-coloured or black spots, $0.1-2(-3) \mathrm{mm}$ long, scattered to sometimes confluent, often moderately swollen. Spores densely packed, tending to 
cohere in irregular groups, variable in shape and size, from globose to irregularly polyhedral or elongate, 5-11 × 6-15(-20) $\mu \mathrm{m}$, yellowish to blackish brown, with a smooth, c. 1-2 $\mu \mathrm{m}$ thick wall. Spore germination results in aseptate basidia bearing (2-)4(-6) short, apical, abaxially curved basidiospores on well-developed sterigmata. Anamorph usually absent (Fig. 14).

On Poaceae:

Dactylis glomerata L., Tehran Prov., Damavand.

Hordeum bulbosum L., Mazandaran Prov., Chalous road, IRAN 8133 F.

Hordeum spontaneum K. Koch, Khuzestan Prov., Behbahan.

Remarks. The great number of synonyms, enumerated above, represent certainly several species which have to be analysed with modern methods.

\section{Macalpinomyces Langdon \& Full.}

23. Macalpinomyces neglectus (Niessl) Vánky, Mycotaxon 89: 106, 2004.

Ustilago neglecta Niessl, in Rabenh., Fgi. eur. no. 1200, 1868. - Sporisorium neglectum (Niessl) Vánky 1985a.

Erysibe panicorum Wallr. var. panici-glauci Wallr. 1833. - Ustilago panici-glauci (Wallr.) G. Winter, in Rabenh. 1881.

Sori in all flowers of an inflorescence, ovoid, often acuminate, $2-4 \mathrm{~mm}$ long, partly enclosed by the glumes, covered by a thin, greyish peridium of fungal and host tissues that ruptures irregularly to expose the dark brown, powdery mass of spores mixed with irregular groups of sterile cells. Columella short, stout, formed of host tissues and fungal cells. Infection systemic. Spores subglobose, ovoid, elongate to slightly irregular, 8-11 $\times 9-14 \mu \mathrm{m}$, medium brown; wall uniformly c. $0.8 \mu \mathrm{m}$ thick, prominently and abundantly echinulate, spore profile serrulate, in SEM the area between the spines with small, rounded, scattered warts. Sterile cells in groups, chains or solitary, in some specimens sparse, smaller than the spores, 5-12 $\mu \mathrm{m}$ long, round or irregular, collapsed in old specimens, hyaline, usually with a light-refractive droplet.

On Poaceae: Setaria glauca (L.) P. Beauv., East Azarbaijan Prov., suburb of Tabriz city.

\section{Microbotryum Lév.}

\section{Key to the Microbotryum species based on host plant taxonomy}

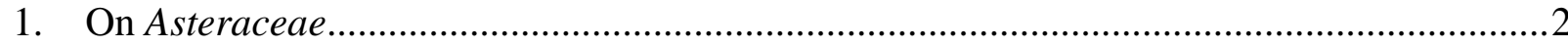

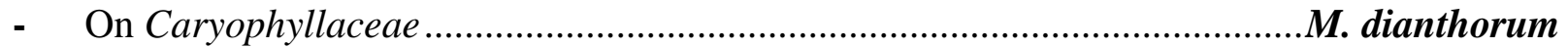

2. On Scorzonera .................................................................................................. scorzonerae

- On Tragopogon........................................................................... tragopogonis-pratensis

24. Microbotryum dianthorum (Liro) H. Scholz \& I. Scholz, Englera 8: 206, 1988.

emend. Lutz, Göker, Piątek, Kemler, Begerow \& Oberwinkler, Mycol. Progr. 4: 234, 2005.

Ustilago dianthorum Liro 1924. - Ustilago violacea (Pers. : Pers.) Rouss. var. dianthorum (Liro)

Durrieu \& Zambett. 1973 (invalidly published).

Ustilago dianthi-arenarii Hammarl., in Gram \& Weber 1942 (nom. nud.).

Sori in swollen anthers. Spore mass violet tinted blackish brown, powdery. Infection systemic; all flowers of an infected plant affected, more rarely, infection semi-systemic and only flowers of certain branches affected. Spores globose to slightly ellipsoidal, 6-11 $\mu \mathrm{m}$ in diam., pale violet; wall reticulate, meshes rounded, $0.5-0.8 \mu \mathrm{m}$ in diam., muri $0.2-0.5 \mu \mathrm{m}$ high, in median view regular, with rounded edges. Spore germination results in 4 -celled $(3+1)$ basidia producing lateral and terminal sessile basidiospores. Basidial cells separating readily and continuing to produce basidiospores or, in nutrient media, yeast cultures (Fig. 15).

On Caryophyllaceae: Dianthus caryophyllus L., Tehran Prov., Varamin (from green house), IRAN 149 \& 8354 F. 
On Dianthus orientalis Adams, Tehran Prov., Shemshak, Dizin road, IRAN 8764 F; Tehran Prov., Karaj-Chalus road, Shahrestanak village, IRAN 8214 F.

On Dianthus tabrisianus Bienert ex Boiss., Ardabil Prov., between Sarab and Ardabil, Farhadgholi area.

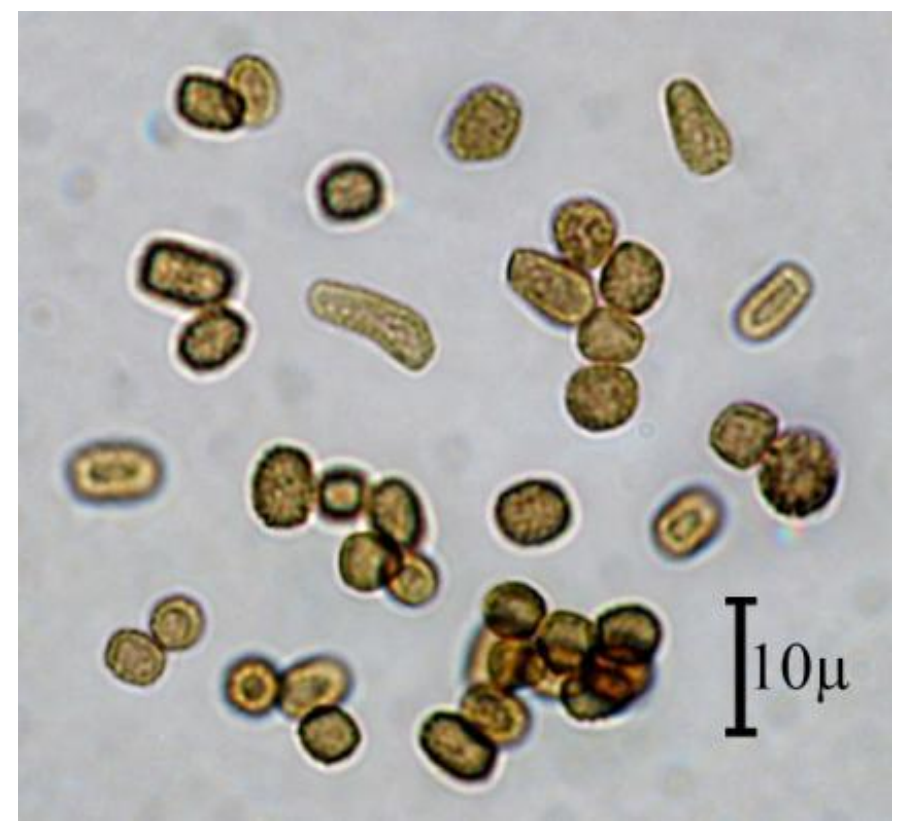

Fig.13 - Farysia thuemenii on Carex riparia (8425)

25. Microbotryum scorzonerae (Alb. \& Schwein.) G. Deml \& Prillinger, in Prillinger, Deml, Dörfler, Laaser \& Lockau, Bot. Acta 104: 10, 1991.

Uredo tragopogi Pers. $\beta \beta$ scorzonerae Alb. \& Schwein. 1805. - Ustilago scorzonerae (Alb. \& Schwein.) J. Schröt., in Cohn 1887. - Bauhinus scorzonerae (Alb. \& Schwein.) R.T. Moore 1992.

Uredo receptaculorum DC. $\beta$ Uredo scorzonerae-humilis DC., in Poiret 1808. - Erysibe receptaculorum (DC.) Wallr. $\beta$ scorzonerae (DC.) Wallr. 1833. - Microbotryum receptaculorum (DC.) Lév. 1847.

Sori in flower heads transforming the florets into blackish violet, powdery spore mass. Infection systemic. Spores globose, subglobose, ovoid to slightly irregular or sometimes elongate, 8-13 × 9$15(-16) \mu \mathrm{m}$, pale brownish violet, often with a light-coloured, nearly hyaline area on one side; wall finely reticulate, 8-12 meshes per spore diam., muri 1-1.5 $\mu \mathrm{m}$ high, in SEM a few warts appear on the lower part of the muri and interspaces. Spore germination of Ustilago-type (Fig. 16).

On Asteraceae: Scorzonera caliculata Boiss., Kermanshah Prov., 17 km SE of Paveh, S slope of Shahu Mt., alt. 2200 m, 15.V.2006, M. Abbasi, R. Fritsch \& M. Keusgen, IRAN 14786 F, HUV 21825 .

26. Microbotryum tragopogonis-pratensis (Pers.) R. Bauer \& Oberw., in Bauer, Oberwinkler \& Vánky, Can. J. Bot. 75: 1311, 1997.

Uredo tragopogi Pers. 1797. - Uredo tragopogi-pratensis Pers. 1801. - Ustilago tragopogonispratensis (Pers.) Roussel 1806. - Ustilago tragopogonis (Pers.) J. Schröt., in Cohn 1887. Bauhinus tragopogonis-pratensis (Pers.) R.T. Moore 1992.

Uredo receptaculorum DC. (nom. nov. illegit. superfl.), var. Uredo tragopogi-pratensis (Pers.) DC., in Poiret, 1808 (as 'trapogi pratensis'). - Caeoma receptaculorum (DC.) Schltdl. 1824 (comb. illegit.). - Ustilago receptaculorum (DC.) Fr. 1832 (comb. illegit.). - Erysibe receptaculorum (DC.) Wallr. var. tragopogonis (Pers.) Wallr. 1833 (comb. illegit.). - Microbotryum receptaculorum (DC.) Lév. 1847 (comb. illegit.). 


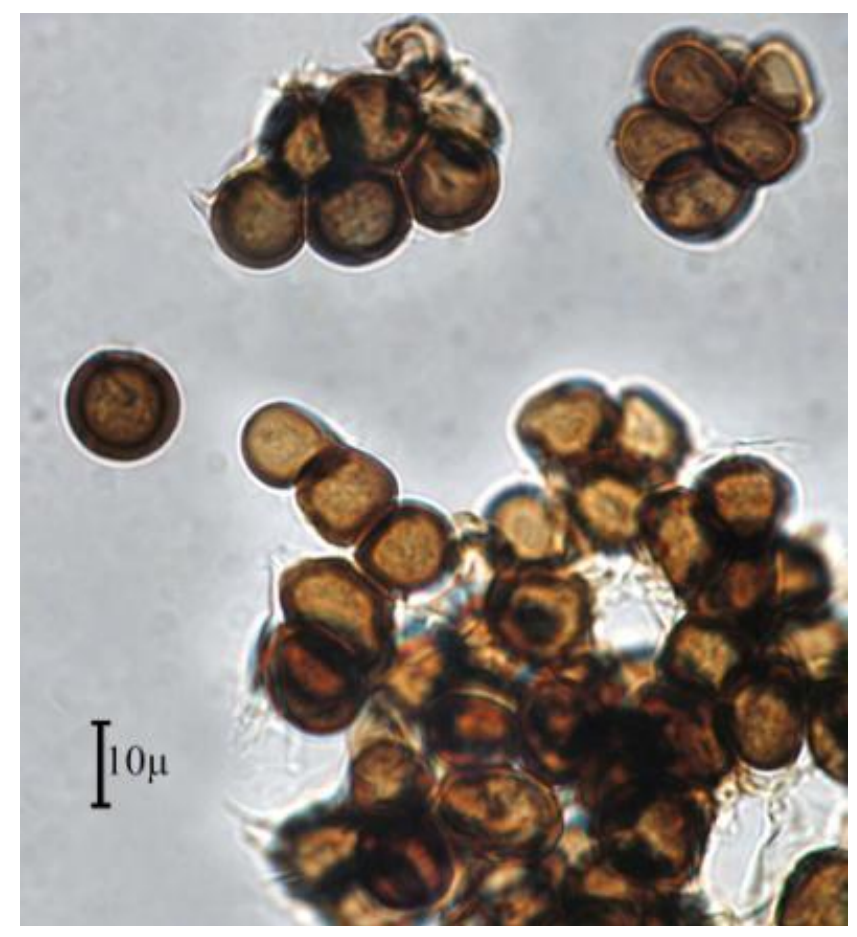

Fig.14 - Jamesdicksonia dactylidis on Hordeum bulbosum (8133)

Sori in flower heads transforming all the florets into a blackish violet, powdery spore mass. Infection systemic; infected plants usually shorter and more ramified than the healthy ones. Spores globose, subglobose, ovoid to pyriform or somewhat irregular, sometimes elongate, 9-17 $\times 13-19 \mu \mathrm{m}$, brownish violet, paler on one side; wall finely, densely reticulate, 8-13 meshes per spore diam., muri 1-1.5 $\mu \mathrm{m}$ high, lower on the paler side, (28-)33-45 on the spore circumference, with blunt, rounded or flattened tips, in SEM interspaces and lower part of the muri with conspicuous, sometimes anastomosed warts. Spore germination results in 4-celled basidia producing laterally and terminally long-ellipsoidal basidiospores that may fuse two by two to form dikaryotic hyphae (Fig. 17).

On Asteraceae: Tragopogon graminifolius DC., Zanjan Prov., between Zanjan \& Mianeh city, IRAN $9542 \mathrm{~F}$.

\section{Moesziomyces Vánky}

27. Moesziomyces bullatus (J. Schröt.) Vánky, Bot. Not. 130: 133, 1977.

Sorosporium bullatum J. Schröt. 1869. - Tolyposporium bullatum (J. Schröt.) J. Schröt. in Cohn, 1887. - Tolypoderma bullata (J. Schröt.) Thirum. \& M.J. O'Brien, in Thirum. \& Neerg. 1978 '1977' (nom. inval.).

Thecaphora globuligera Berk. \& Broome 1880. - Tolyposporium globuligerum (Berk. \& Broome) Ricker 1905. - Moesziomyces globuligerus (Berk. \& Broome) Vánky 1977. - Tolypoderma globuligera (Berk. \& Broome) Thirum. \& M.J. O'Brien, in Thirum. \& Neerg. 1978 '1977' (nom. inval.).

Testicularia leersiae Cornu 1883.

Tolyposporium penicillariae Bref. 1895. - Moesziomyces penicillariae (Bref.) Vánky 1977. Tolyposporidium penicillariae (Bref.) Thirum. \& Neerg. 1978 '1977'.

Tolyposporium minus J. Schröt., in Henn. 1896.

Ustilago verrucosa J. Schröt., in Henn. 1896.

Tolyposporium senegalense Speg. 1915.

Tolyposporium evernium Syd., in Syd. \& S. Ahmad 1939. - Moesziomyces evernius (Syd.) Vánky 1977. - Tolyposporidium evernium (Syd.) Thirum. \& Neerg. 1978 '1977'. 
Tolyposporium paspali Langdon 1948.

Sori in scattered ovaries, globose to ovoid, 2-4(-5) $\mathrm{mm}$ long, covered by a smooth, initially green, later brown peridium of host tissue. Infection floral, through the stigma and style. The entire sorus may fall off the host or first the peridium ruptures irregularly exposing a granular, dark brown mass of spore balls; columella absent. Hyphae intracellular. Spore balls variable in shape and size, globose, subglobose, ovoid, elongate to irregular, (35-)50-240 $\times(40-) 60-320 \mu \mathrm{m}$ in diam., dark brown, opaque, composed of numerous, up to several hundred spores, united by sterile fungal cells and spore processes, no cortex of sterile cells. Spores globose, ovoid to irregular, slightly polyhedral, subhyaline to pale yellowish brown, 6.5-9(-10) $\times 7-12(-13) \mu \mathrm{m}$ in diam., usually with several, more or less well-developed extensions (spore processes) and also remnants of the broken walls of the sterile cells; wall $0.5-0.7 \mu \mathrm{m}$ thick, smooth. Sterile cells empty at maturity, thin-walled, $0.1-0.2$ $\mu \mathrm{m}$, appearing as irregular meshes or wings on the spore surface when the spores are separated. Spore germination results in septate basidia producing basidiospores laterally and terminally, on well-developed sterigmata (Fig. 18).

On Poaceae: Echinochloa crus-galli (L.) P. Beauv., Gilan Prov., suburb of Astara city, in rice field; Gilan Prov., suburb of Rasht city, IRAN 1352 F.

\section{Schizonella J. Schröt.}

28. Schizonella cocconii (Morini) Liro, Ann. Acad. Sci. Fenn., Ser. A, 42(1): 52, 1938.

Tolyposporium cocconii Morini 1884.

Sori in leaves, mostly epiphyllous, as black, blister-like, intraepidermal streaks of various lengths, initially covered by the thin layer of cuticle that ruptures longitudinally to expose the initially agglutinate, later granular-powdery spore mass. Spore balls irregular, composed of 2-20 (or more?) spores, rather readily separating into single spores or pairs of spores. Spores heterogeneous in form and size, globose, hemiglobose, ovoid to elongate and subangular, irregular, 6-10 $(-12) \times 8-16(-18)$ $\mu \mathrm{m}$, light olive-brown to dark reddish brown; wall smooth, thin and light on the contact side, and thick, dark, often finely verruculose on the opposite side. Spore germination of Ustilago-type (Fig. 19).

On Cyperaceae: Carex halleriana Asso, East Azarbaijan Prov., Arasbaran protected area, Kaleibar city, Makidi valley, IRAN 13967 F.

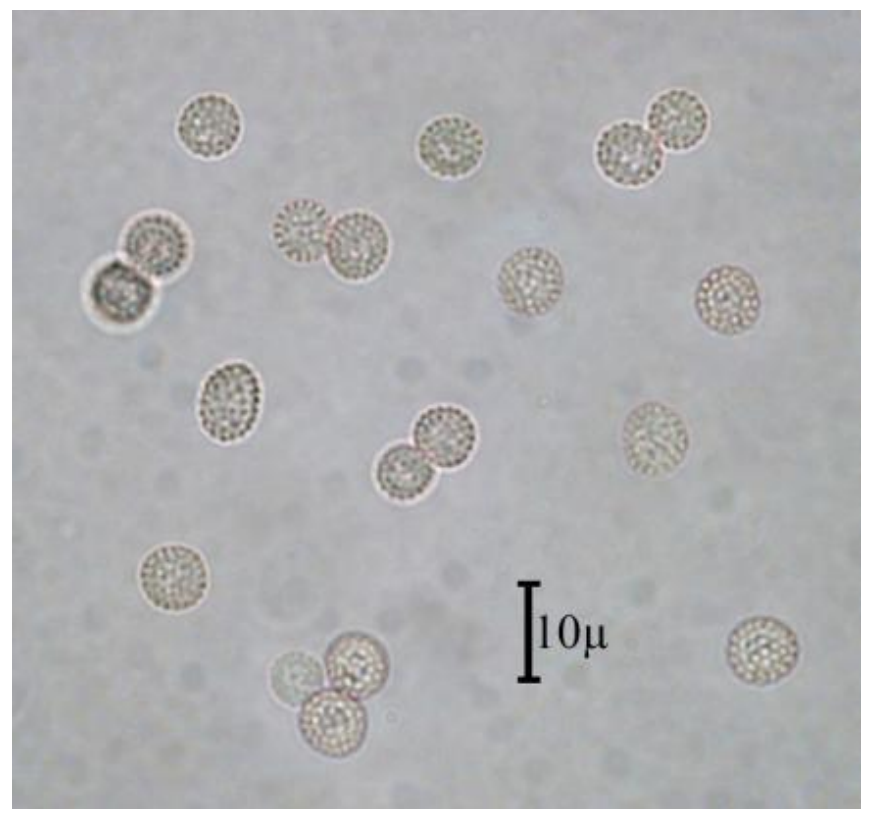

Fig.15 - Microbotryum dianthorum on Dianthus caryophyllus (149) 


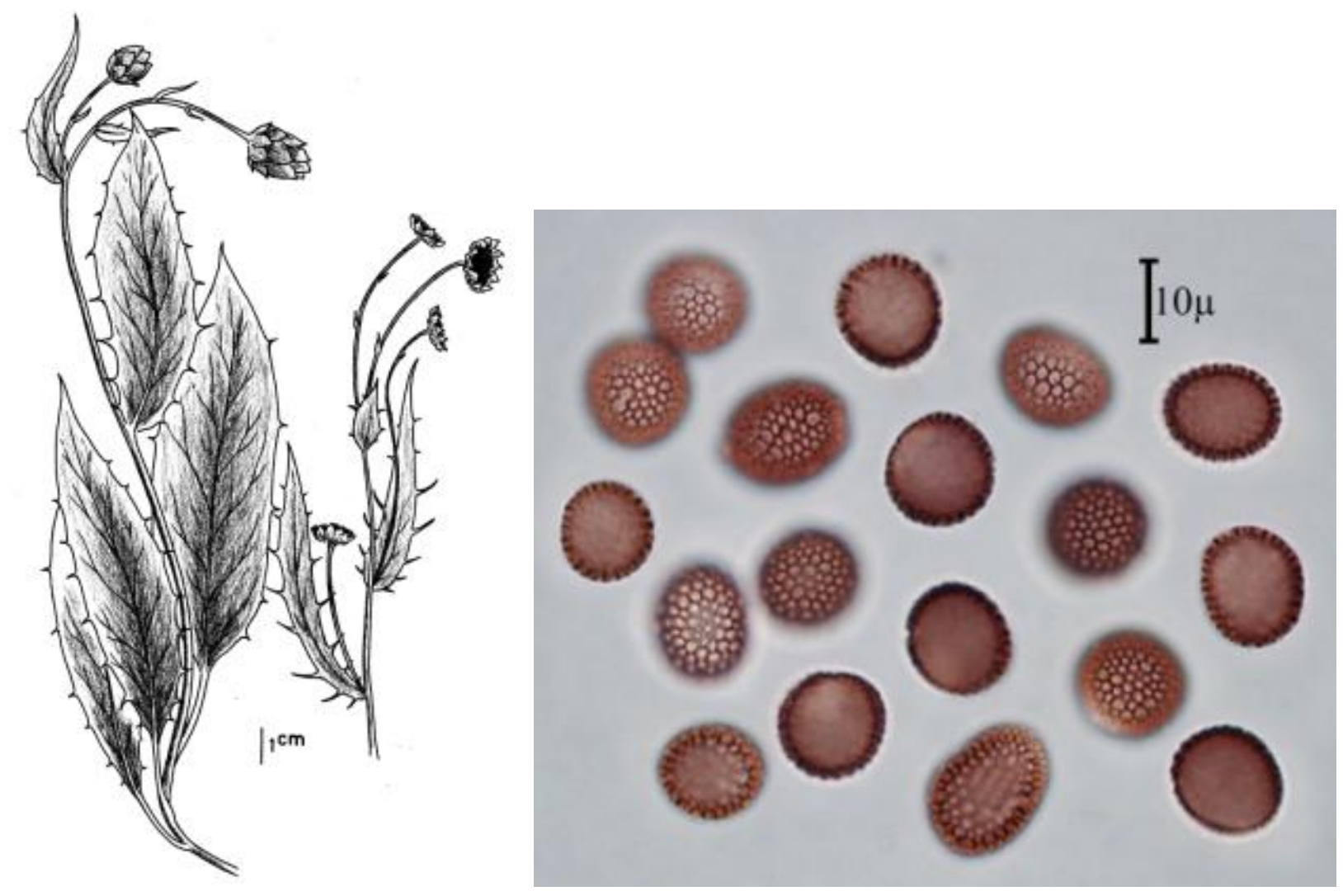

Fig.16 - Microbotryum scorzonerae on Scorzonera caliculata (14786), infected plant (left) and teliospores (right)

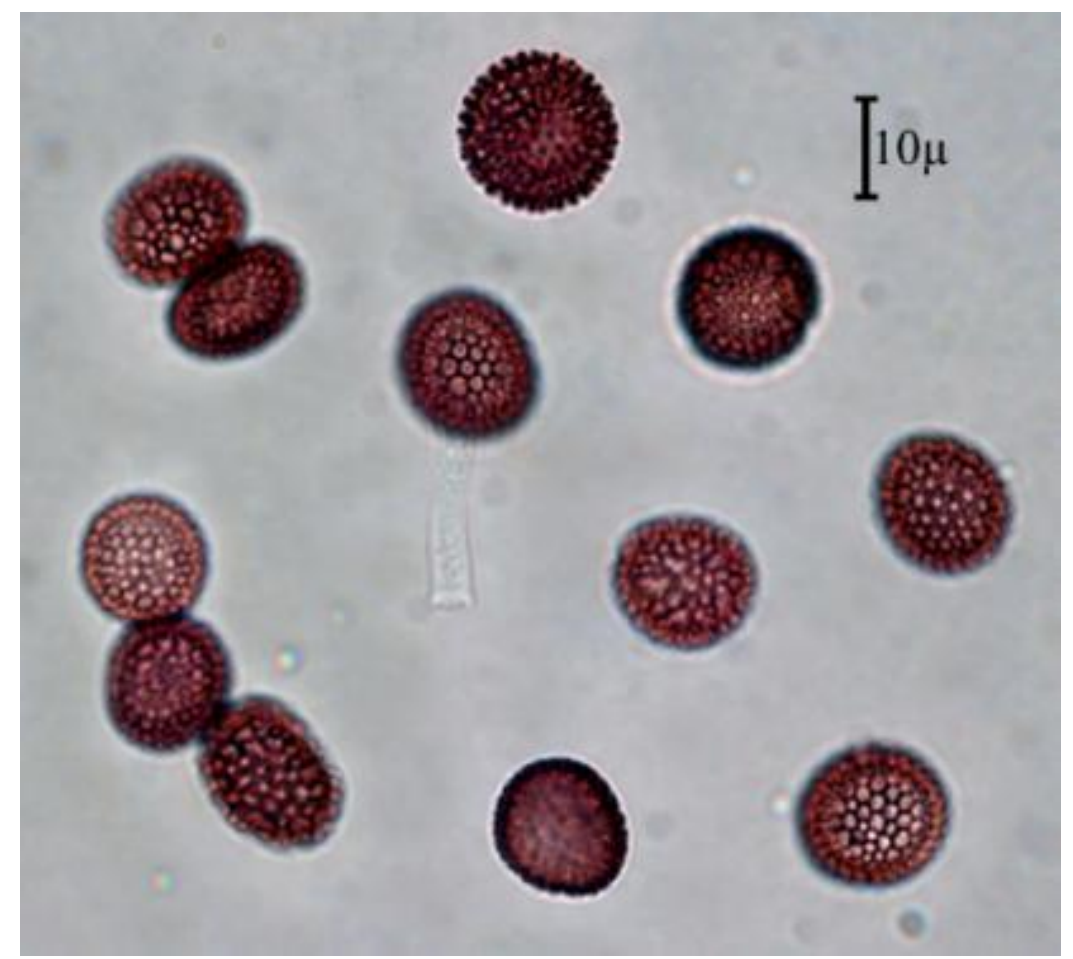

Fig.17 - Microbotryum tragopogonis-pratensis on Tragopogon graminifolius (9542) 


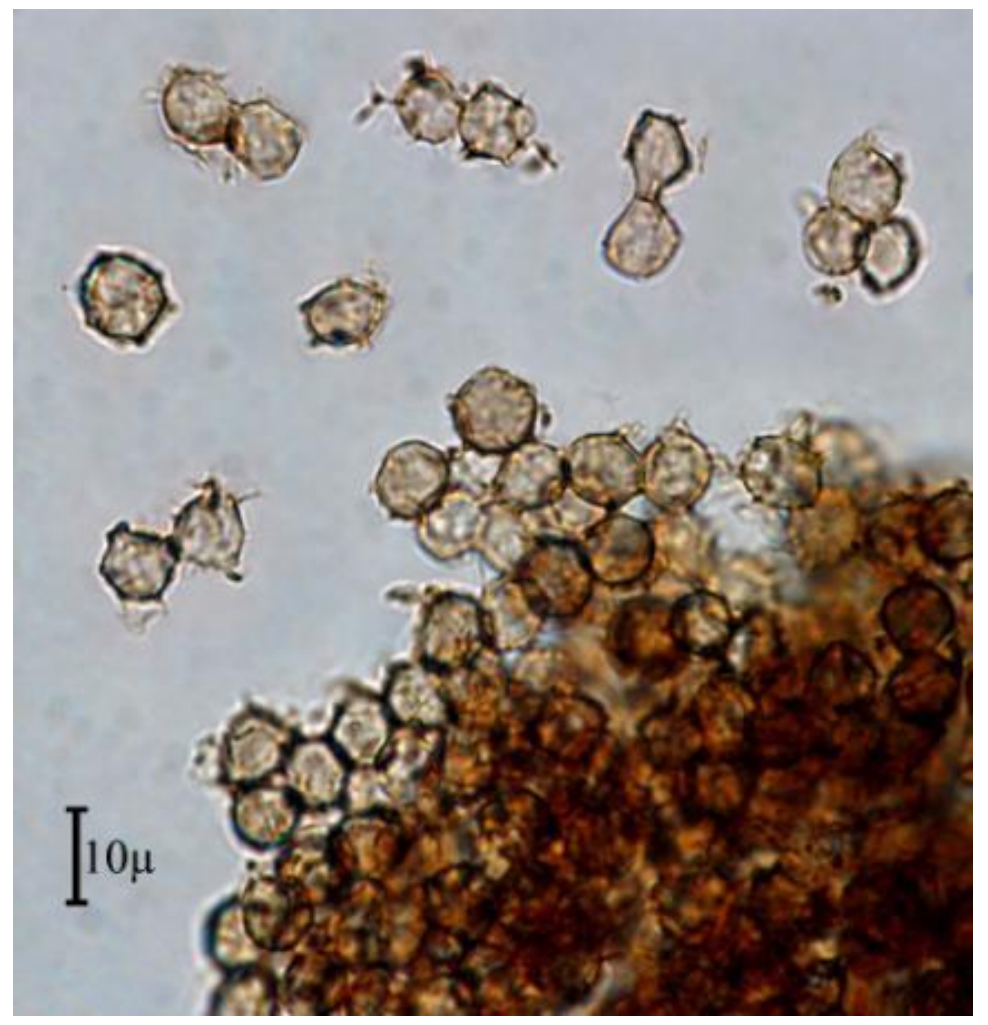

Fig.18 - Moesziomyces bullatus on Echinochloa crus-galli (1352)

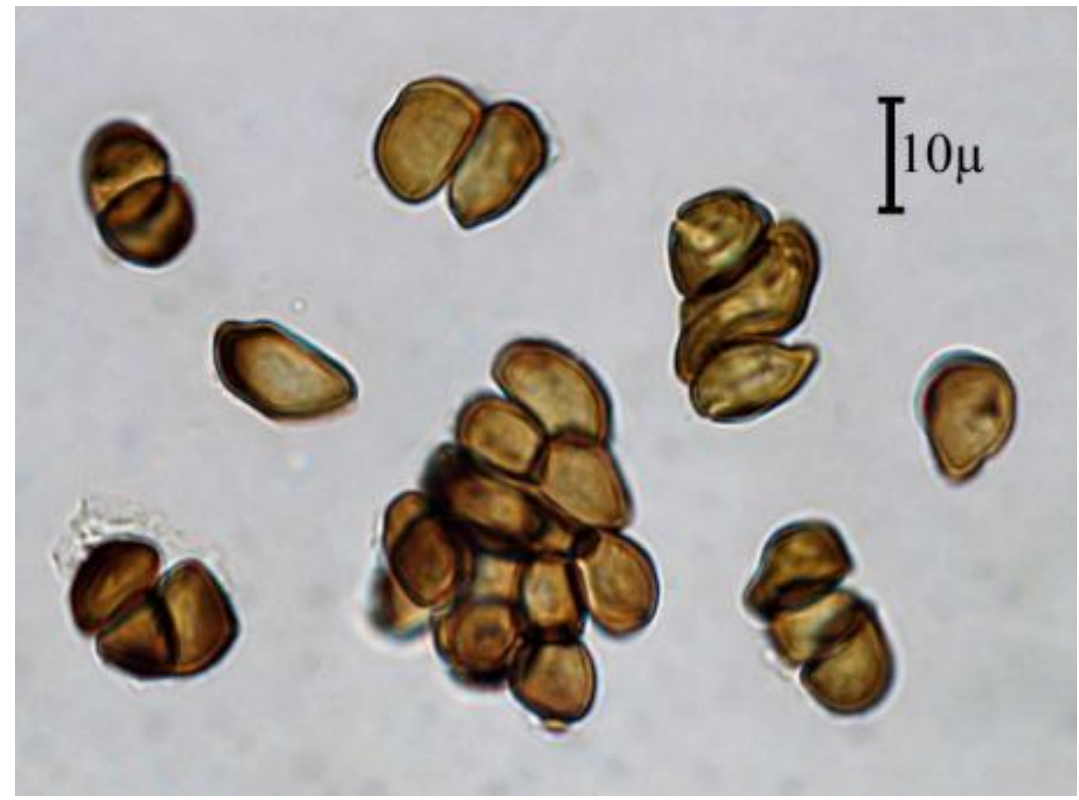

Fig.19 - Schizonella cocconii on Carex halleriana (13967)

Sporisorium Ehrenb. ex Link

Key to the Sporisorium species based on host plant taxonomy

1. On Cymbopogon

S. lanigeri

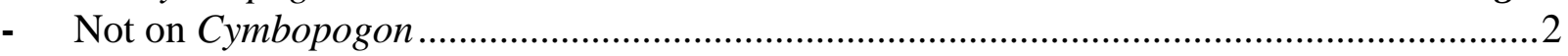

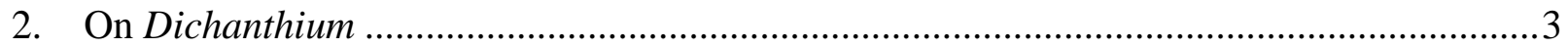

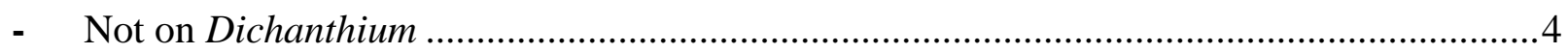

3. Spores 7.5-11 $\mu \mathrm{m}$ long, finely, densely punctate-verruculose ....................S. andropogonis 
- $\quad$ Spores 9-13 $\mu \mathrm{m}$ long, densely verruculose-echinulate

5. On Imperata S. schweinfurthianum

- Not on Imperata

6. On Lasiurus S. desertorum

- Not on Lasiurus 7

7. On Panicum. S. destruens

- Not on Panicum .8

8. On Pennisetum. S. penniseti

- Not on Pennisetum ..9

9. On Schismus

S. aegyptiacum

- Not on Schismus 10

10. On Sorghum. .11

- Not on Sorghum .14

11. Sori in whole inflorescence S. reilianum

- Sori in ovaries or spikelets. .12

12. Sori in considerably hypertrophied ovaries; spores $9.5-15 \mu \mathrm{m}$ long S. ehrenbergii

- $\quad$ Sori in the spikelets; spores smaller .13

13. Peridium thin, dehiscent; spores 7.5-10 $\mu \mathrm{m}$ long, verruculose echinulate S. cruentum

- Peridium thick, persistent; spores 5-8(-8.5) $\mu \mathrm{m}$ long, apparently smooth to sparsely punctate or very finely verruculose.

14. On Spodiopogon S. sorghi

- Not on Spodiopogon S. persicum

15. On Stipagrostis .15

- On Tricholaena S. aristidae-lanuginosae S. tricholaenae

29. Sporisorium aegyptiacum (A.A. Fisch. Waldh.) Vánky, Mycotaxon 33: 371, 1988b (as 'aegypticum').

Ustilago aegyptica A.A. Fisch. Waldh. 1879a, b.

Ustilago schismi Bubák 1914.

Sori in spikelets destroying the innermost floral organs leaving intact the glumes and the distal part of the inner floral envelopes, c. $1 \times 2-3 \mathrm{~mm}$, hidden by the glumes, initially covered by a yellowish brown peridium that ruptures irregularly from its basal part, flakes away together with the intact distal part of the floral envelopes, exposing the dark brown, powdery mass of spores and sterile cells surrounding a 2-3 mm long, simple, central columella sometimes with short lateral branches. Infection systemic; all spikelets of a plant infected. Diseased plants more or less stunted. Spores when mature single, subglobose, ellipsoidal to slightly irregular, 9-12 $\times 10.5-14.5 \mu \mathrm{m}$, often flattened, 6.5-8 $\mu \mathrm{m}$ wide, yellowish brown; wall even, $0.8 \mu \mathrm{m}$ thick, densely, finely echinulate, spore profile almost smooth to finely serrulate. Sterile cells in irregular groups or chains or single, variable in shape and size, subglobose, ellipsoidal, elongate or slightly irregular, 5-6.5 ×5-10.5 $\mu \mathrm{m}$, hyaline, contents homogeneous; wall thin, c. $0.5 \mu \mathrm{m}$, smooth. Spore germination results in 4-celled basidia (in a $3+1$ arrangement), producing basidiospores or, after fusion of two compatible basidial cells, dikaryotic hyphae (Fig. 20).

On Poaceae: Schismus arabicus Nees, Golestan Prov., $6 \mathrm{~km} \mathrm{~S}$ of Maraveh tappeh village, IRAN 7232 F; Gilan Prov., c. $85 \mathrm{~km} \mathrm{~S}$ of Rasht city, east end of the lake White River dam, IRAN $7233 \mathrm{~F}$.

30. Sporisorium andropogonis (Opiz) Vánky, Symb. Bot. Upsal. 24(2): 113, 1985a.

Uredo (Ustilago) andropogonis Opiz 1824 (as 'andropogi'). - Sphacelotheca andropogonis (Opiz) Bubák 1912. - Cintractia andropogonis (Opiz) Kochman 1936. 
Ustilago ischaemi Fuckel 1860. - Cintractia ischaemi (Fuckel) Syd. \& P. Syd. 1901. Sphacelotheca ischaemi (Fuckel) G.P. Clinton 1902.

Ustilago cylindrica Peck 1882.

Sorosporium platense Hirschh. 1941. (as 'platensis'). - Sphacelotheca platensis (Hirschh.) Hirschh. 1986.

Sphacelotheca chloridis Mundk. 1944a.

Ustilago bothriochloae-intermediae Padwick 1946. - Sphacelotheca bothriochloae-intermediae (Padwick) Naras., in Thirum. \& Pavgi 1956.

Sphacelotheca heteropogonis-triticei L. Ling 1950.

Sorosporium baluchistani S. Ahmad 1956.

Sphacelotheca bothriochloae Y.C. Wang 1962. (later homonym, not Zundel).

Sori usually destroying the whole inflorescence, rarely confined to the spikelets, cylindrical or bifurcate at their distal part, 1-10 mm wide, $1.5-7 \mathrm{~cm}$ long, partly hidden by the terminal leaf sheath, initially covered by a well-developed, yellowish brown peridium that ruptures irregularly and flakes away exposing the dark brown, semi-agglutinated to powdery mass of spore balls, spores and groups of sterile cells surrounding a simple or ramified, irregular columella of the length of the sorus, the remnants of the floral axis and branches. Infection systemic. Spore balls loose, subglobose, ellipsoidal, elongate or irregular, 20-100 × 40-160 $\mu \mathrm{m}$, dark reddish brown, composed of tens of spores that separate very easily. Spores when mature single, globose, ovoid, ellipsoidal to slightly irregular, $(6.5-) 7-10 \times 7.5-11 \mu \mathrm{m}$, light olive-brown; wall even, 0.5-1 $\mu \mathrm{m}$ thick, finely, densely punctate-verruculose, spore profile smooth to wavy, in SEM spores minutely echinulate, between the spines finely and densely verruculose. Sterile cells in irregular groups or chains among the spores and also forming the peridium, globose to irregularly polyhedral, flattened on contact sides, 6-16(-22) $\mu \mathrm{m}$ long, subhyaline to yellow tinted, with numerous droplets, collapsed in old specimens; wall even, 0.5-1 $\mu \mathrm{m}$ thick, smooth. Spore germination results in 4-celled basidia on which lateral and terminal basidiospores are produced (Fig. 21).

On Poaceae: Dichanthium annulatum Stapf, Hormozgan Prov., Biaban, Gattan, IRAN 4424 F; Hormozgan Prov., Bandar-Abbas, Kahoorestan, IRAN 11243 F; Tehran Prov., Tehran city, Evin area, IRAN 7954 F.

On Dichanthium ischaemum (L.) Roberty (Andropogon ischaemum L.; Bothriochloa ischaemum (L.) Keng), Ghazvin Prov., Ebrahimabad village; Tehran Prov., Fasham city; Tehran Prov., Karaj-Chalus road, Gachsar; Tehran Prov., Taleghan city; Fars Prov., Shiraz city; Golestan Prov., Golestan National Park, Tange rah area, IRAN 11020 F; Yazd Prov., Banadaksadat village, IRAN 13877 F; Tehran Prov., Chalus road, Kalha village, IRAN 10992 F; Tehran Prov., Karaj city, IRAN 4422 \& 4423 F; Gilan Prov., 1 km N of Lake White River dam, IRAN 7265 F.

\section{Sporisorium aristidae-lanuginosae (Maire) Vánky, Mycotaxon 78: 304, 2001.}

Sphacelotheca aristidae-lanuginosae Maire 1906.

Sori comprise the whole inflorescence destroying all of them in a plant, ovoid with acute tip, c. $0.5 \times$ $1 \mathrm{~cm}$, partly hidden by leaf sheaths, initially covered by a whitish to pale brown peridium that ruptures exposing the blackish brown, semi-agglutinated to powdery mass of spore balls and spores surrounding numerous, stout, 0.5-1 cm long columellae. Spore balls subglobose, ellipsoidal, elongate to irregular, $25-70 \times 30-80 \mu \mathrm{m}$, dark reddish brown, composed of tens of easily separating spores. Spores subglobose, ellipsoidal, elongate, usually subpolyhedrally irregular, 8-12 × 9.513.5(-15) $\mu \mathrm{m}$, yellowish brown; wall evenly thick, c. $1 \mu \mathrm{m}$, finely, densely verruculose, spore profile smooth to very finely serrulate. Sterile cells absent (Fig. 22).

On Poaceae: Stipagrostis plumosa (L.) Munro ex T. Anderson, Yazd Prov., Anar city, Bahramabad village, IRAN 8300 F, HUV 15311; Esfahan Prov., $45 \mathrm{~km} \mathrm{NW}$ of Kashan, road towards Qom, 5.VIII.1974, leg. Wendelbo, Rafii \& Riazi, Herb. TARI 14441, HUV 7480; Kerman Prov., Rafsanjan city, 21.IV.1946, leg. Kriukhin, IRAN 4427 F; Sistan va Baluchestan Prov., Zabol, Dashtak Police Station, IRAN 14881 F. 
Remarks - This species was treated in Ershad (2001: 99) under the name Sporisorium saharianum (Trotter) Karatygin.

32. Sporisorium cruentum (J.G. Kühn) Vánky, Symb. Bot. Upsal. 24(2): 115, 1985a.

Ustilago cruenta J.G. Kühn 1872. - Sphacelotheca cruenta (J.G. Kühn) Potter 1912.

Ustilago sorghi Passerini, in Thümen 1873.

Endothlaspis sorghi Sorokin 1890. - Cintractia sorghi (Sorokin) de Toni, in Sacc. 1888. Sphacelotheca sorokiniana (Sorokin) Cif. 1928 (nom. nov.).

Sphacelotheca holci H.S. Jacks. 1934 (n.v.).

Sori in all spikelets of the congested and stunted panicle, covered by a delicate peridium which easily ruptures to reveal the blackish brown, powdery spore mass, mixed with groups of sterile cells and a well-developed columella. Columella composed of host tissues permeated by hyphae producing spores and sterile cells. Mature spores single or in irregular, loose groups. Spores globose to ovoid, $6.5-8.5 \times 7.5-10 \mu \mathrm{m}$, light olive-brown, finely and sparsely verruculose to minutely echinulate. Sterile cells much larger than the spores $(8-16 \mu \mathrm{m}$ long), in small, irregular groups or chains, hyaline or pale yellow, smooth. Spore germination results in 4-celled basidia which in WA give rise to hyphae, in nutrient media to ovoid or elongate basidiospores which bud (Fig. 23).

On Poaceae: Sorghum bicolor (L.) Moench. Only one record made by Khabiri (1956) from unknown locality.

On Sorghum halepense (L.) Pers., Kohgiluyeh va Buyerahmad Prov., Yasouj city, Babamaidan road, Tange-Tamoradi area, IRAN 8316 F; Ardabil Prov., Moghan city, Ultan village, IRAN 8212 F; Lorestan Prov., Boroujerd city, Khaayan village, IRAN 8238 F; Lorestan Prov., Khorramabad city, IRAN 4421 \& 6585 F; Fars Prov., Darab city, IRAN 11538 F; Kermanshah Prov., Sahneh, IRAN 6634 F; Khuzestan Prov., Dezful, Safiabad, IRAN 6586 F; Yazd Prov., at Yazd city, Shahedieh, 14576 F; Lorestan Prov., between Khorramabad city \& Alashtar city, IRAN 8757 F; Yazd Prov., Marvast city, IRAN 13878 F; Tehran Prov., Tehran city, Evin area, IRAN 9563 F; Fars Prov., Malusjan village, IRAN 13282 F; Kermanshah Prov., Bisotun area, IRAN 2534 F; West Azarbaijan Prov., IRAN 12835 F; Fars Prov., Shiraz city, Bagh-e Farhangshahr, IRAN 13188 F; Fars Prov., Sivand dam, Sar-e asiab spring, IRAN 13190 F; Esfahan Prov., Semirom city, Padena village, Bideh village, IRAN 11192 F, Guilan Prov., Lushan, IRAN 8237 F.

On Sorghum sp., Kermanshah Prov., Ghasre Shirin city, IRAN 4426 F; Yazd Prov., Abarkooh city, IRAN 13929 F.

33. Sporisorium desertorum (Thüm.) Vánky, Mycotaxon 74: 169, 2000.

Sorosporium desertorum Thümen 1879.

Sorosporium lasiuri S. Ahmad 1969.

Sorosporium lasiuri Zambett. 1970 (later homonym and nom. inval.).

Sori destroying the whole inflorescence (raceme), more rarely only a part of it or only a few spikelets in the raceme, fusiform, 1-2(-3) $\times 10-35 \mathrm{~mm}$, partly hidden by leaf sheaths, covered by a thick, brown peridium that ruptures irregularly exposing the dark brown mass of spore balls, spores, sterile cells and several long, filiform columellae. All racemes or only some of them in a plant may be affected. Spore balls globose to irregularly ellipsoidal, 35-60(-75) $\mu \mathrm{m}$ long, dark reddish brown, easily separating into single spores. Spores globose, subglobose, ellipsoidal, slightly irregular, 8.5$10.5 \times 9.5-12(-13) \mu \mathrm{m}$, reddish brown; wall uneven, $1-1.5 \mu \mathrm{m}$ thick, thickest at the angles, outer spores coarsely verrucose-echinulate, especially on their free surface, inner spores finely punctate. Sterile cells single, globose, subglobose, ellipsoidal, usually more or less flattened, 8-16 $\mu \mathrm{m}$ long, hyaline; wall 1-1.5 $\mu \mathrm{m}$ thick, smooth (Fig. 24).

On Poaceae: Lasiurus sindicus Henrard (L. hirsutus (Forssk.) Boiss.; Coelorachis hirsuta (Forssk.) Brongn.); Sisitan va Baluchestan Prov., Chabahar city, 2 km of Pasaband city towards Goatar port, IRAN 11288 F, HUV 21822.

Remarks - Sporisorium desertorum, treated in Ershad (2001: 83) as S. andropogonis, is known from a few countries in Africa (Chad, Egypt, Mauritania) and in S Asia (India, Pakistan). 
34. Sporisorium destruens (Schltdl.) Vánky, Symb. Bot. Upsal. 24(2): 115, 1985a.

Caeoma destruens Schltdl. 1824. - Uredo destruens (Schltdl.) Duby 1830. - Tilletia destruens (Schltdl.) Lév. 1847. - Ustilago destruens (Schltdl.) Rabenh. 1857. - Anthracocystis destruens (Schltdl.) Bref. 1912. - Sphacelotheca destruens (Schltdl.) J.A. Stev. \& A.G. Johnson 1944.

Ustilago panici-miliacei (Pers.) G. Winter 1881. - Sorosporium panici-miliacei (Pers.) Takah. 1902. - Sphacelotheca panici-miliacei (Pers.) Bubák 1912. - Uredo segetum var. panici-miliacei Pers. 1801. - Uredo carbo var. panici-miliacei (Pers.) DC. 1815.

Uredo segetum Pers. var. Uredo panici-miliacei Pers. 1801. - Uredo carbo var. panici-miliacei

(Pers.) DC. 1815. - Erysibe panicorum var. panici-miliacei (Pers.) Wallr. 1833. - Ustilago panici-miliacei (Pers.) G. Winter 1881. - Sorosporium panici-miliacei (Pers.) Takah. 1902. Sphacelotheca panici-miliacei (Pers.) Bubák 1912.

Sorosporium manchuricum S. Ito 1935. - Sphacelotheca manchurica (S. Ito) Y.C. Wang 1962. Sphacelotheca lioui W.Y. Yen 1937a.

Sori destroying the whole inflorescence, ovoid to elongate, $0.5-2 \times 3-6 \mathrm{~cm}$, partly concealed by leaf sheaths and covered by a well-developed, whitish peridium that ruptures irregularly and flakes away to expose the dark brown, dusty spore mass, sterile cells and numerous, long, filiform columellae composed of host tissues, mainly vascular bundles, and hyphae. Infection systemic, initiated in seedling stage of the hosts. Spore balls loose, ephemeral. Spores when mature single, subglobose, ovoid to elongate or irregular, $7-9 \times 8-13 \mu \mathrm{m}$, pale reddish brown; wall even or nearly so, c. $0.5 \mu \mathrm{m}$ thick, smooth to very finely punctate, spore profile smooth, in SEM very finely and densely verruculose. Sterile cells among the spores subhyaline or pale yellow, thin-walled, almost the size of the spores. Spore germination results in 4-celled basidia. In water, basidial cells fuse and produce hyphae, in nutrient media abundant basidiospores are produced (Fig. 25).

On Poaceae: Panicum miliaceum L., Fars Prov., Abadeh city, IRAN 4420 F; Khorasan Prov., Mashhad city, Tous city, IRAN 8313 F; Esfahan Prov., suburb of Esfahan city, IRAN 10320 F.

35. Sporisorium ehrenbergii (J.G. Kühn) Vánky, Mycotaxon 38: 270, 1990.

Sorosporium ehrenbergii J.G. Kühn 1877. - Tolyposporium ehrenbergii (J.G. Kühn) Pat. 1903. Tolyposporium filiferum Busse 1905. - Sorosporium filiferum (Busse) Zundel 1930b.

Sorosporium andropogonis-sorghi S. Ito 1935.

Sori in occasional ovaries, cylindrical, elongate, often curved, $0.3-1 \times 1-3 \mathrm{~cm}$, covered by a thick, smooth, whitish to yellowish brown peridium which fissures apically or longitudinally to expose the black, granular mass of spore balls mixed with several, long, often ramified, filiform columellae, composed of host tissues and fungal cells. Peridium composed of chains of ovoid to $10-50 \mu \mathrm{m}$ long, cylindrical, hyaline fungal cells. Spore balls variable in shape and size, subglobose to oblong or irregular, 40-100(-150) $\mu \mathrm{m}$ long, rather permanent, many-spored, dark brown, opaque. Spores globose or, more commonly subpolyhedral, 9-12 $\times 9.5-15 \mu \mathrm{m}$, dimorphic. Outer spores dark brown, verrucose on the free surface. Inner spores pale yellowish brown, thin-walled, smooth. Spore germination results in 2-4-celled basidia, producing laterally and terminally ovoid or fusiform basidiospores (Fig. 26).

On Poaceae: Sorghum bicolor (L.) Moench (S. vulgare Pers.), Khuzestan Prov., Dezful city; East Azarbaijan Prov., Mianeh; Khuzestan Prov., Ramin city; Zanjan Prov.; Esfahan Prov., Zavareh city; Esfahan Prov., Ardestan city, Gazla village, IRAN 10322 F; Kerman Prov., Jazmurian area, Kangru village, IRAN 4400 F; Sistan va Baluchestan, Iranshahr city, Bampoor city, IRAN 4399 F.

36. Sporisorium foveolati (Maire) Vánky, Mycotaxon 33: 367, 1988b.

Sphacelotheca foveolati Maire 1931a.

Sori destroying the whole inflorescence, 1-2 $\times 5-15 \mathrm{~mm}$, partly hidden by the uppermost leaf sheath, initially covered by a yellowish brown peridium which flakes away revealing the dark brown, semi-agglutinated to powdery mass of spores and sterile cells surrounding a usually flagelliform columella. Spores rounded subpolyhedral, often irregular, 8-11 $\times 9-13 \mu \mathrm{m}$, olive- 
brown; wall even, $0.5-0.8 \mu \mathrm{m}$ thick, densely verruculose-echinulate, spore profile wavy to finely serrulate. Sterile cells in loose, irregular groups or solitary, globose, ellipsoidal to slightly irregular, 9-20 × 13-22 $\mu \mathrm{m}$, pale yellowish brown; wall 1.5-3 $\mu \mathrm{m}$ thick, smooth (Fig. 27).

On Poaceae: On Dichanthium annulatum (Forssk.) Stapf, Hormozgan Prov., Biaan, Gattan, 4.III.1949, IRAN 4424 F; Hormozgan prov., Bandar, Abbas, Kahoorestan, 5.III.2001, IRAN 11343 F.

On Dichanthium foveolatum (Delile) Roberty (Andropogon foveolatus Delile; Eremopogon foveolatus (Delile) Stapf), Hormozgan Prov., Persian Gulf, Lavan Island, IRAN 4425 F, HUV 21819.

Remarks - This smut, known from several countries in Africa, is new to Iran, and probably also to Asia. In Ershad (2001: 82) it was treated as Sporisorium andropogonis.

37. Sporisorium lanigeri (Magnus) Ershad, Iran. J. Pl. Pathol. 33: 59, 1998 '1997'.

Ustilago lanigeri Magnus 1899. - Sphacelotheca lanigeri (Magnus) Maire, in Zundel 1930b. Sporisorium lanigeri (Magnus) Vánky 2003 (comb. superfl.).

Ustilago furcata Pat. \& Har. 1900. - Sphacelotheca furcata (Pat. \& Har.) Pat. \& Har., in Har. \& Pat. 1909.

Ustilago schoenanthi Syd., P. Syd. \& E.J. Butler 1906. - Sphacelotheca schoenanthi (Syd., P. Syd. \& E.J. Butler) Zundel 1930b.

Sphacelotheca moggii Zundel 1930b.

Sphacelotheca concentrica Zundel 1930b.

Sorosporium pretoriense Zundel 1930b.

Sphacelotheca natalensis Zundel 1930b.

Sphacelotheca columellifera (Tul. \& C. Tul.) W.Y. Yen 1937b. (later homonym, not Cif. 1928). Sphacelotheca yenii Zundel 1939.

Sphacelotheca cymbopogonis W.Y. Yen 1938a.

Sphacelotheca consueta Syd., in Syd. \& S. Ahmad 1939.

Sorosporium ladharense Syd. in Syd. \& S. Ahmad 1939.

Sphacelotheca cymbopogonis-colorati Mundk. \& Thirum., in Thirum. \& Mundk. 1951. Sporisorium cymbopogonis-colorati (Mundk. \& Thirum.) Kakish. \& Y. Ono, in Nakaike \& Malik (eds.) 1993.

Sphacelotheca mutila Mundk. \& Thirum., in Thirum. \& Mundk. 1951.

Sporisorium martinii Bag \& D.K. Agarwal 2001 (as 'martinae').

Sori destroying all racemes of an inflorescence, more or less hidden by the enveloping spathae, cylindrical, tapering at the apex, 1-2 $\times 5-16 \mathrm{~mm}$, initially covered by a pale brown, thick peridium that ruptures irregularly exposing the dark brown, semi-agglutinated to powdery mass of spore balls, spores and sterile cells surrounding a stout, tapering, rarely flagelliform, sometimes bifurcate, central columella. Spore balls globose, ellipsoidal to irregular, 30-120 × 40-140 $\mu \mathrm{m}$, dark reddish brown to opaque, composed of numerous spores that separate under pressure. Spores globose, subglobose, ellipsoidal to slightly irregular, often slightly flattened, 5.5-8 ×6-9 $\mu \mathrm{m}$, yellowish to reddish brown; wall evenly thick, 0.5-1 $\mu \mathrm{m}$, apparently smooth to finely, moderately densely verruculoseechinulate, spore profile smooth to finely wavy. Sterile cells in irregular groups, individual cells subglobose, ellipsoidal to irregular, 7-20 $\mu \mathrm{m}$ long, hyaline to pale yellowish brown tinted; wall 1$1.5 \mu \mathrm{m}$ thick, smooth (Fig. 28).

On Poaceae: Cymbopogon olivieri (Boiss.) Bor, Kerman Prov., Tagh-ali Mt.; Hormozgan Prov., Minab, IRAN 12374 F, HUV 21970; Hormozgan Prov., Jask, Bashagard area, IRAN 12399 F, HUV 21983, also in Vánky, Ust. exs. no. 1291.

38. Sporisorium penniseti (Rabenh.) Ershad, Iran. J. Pl. Pathol. 30: 18, 1994.

Ustilago penniseti Rabenh. 1871. - Sphacelotheca penniseti (Rabenh.) Reichert 1921.

Ustilago pappiana Bacc. 1906. - Sorosporium pappianum (Bacc.) L. Ling 1953.

Sorosporium catharticum Maire 1931b. - Sporisorium catharticum (Maire) Vánky 1989. 
Sphacelotheca panjabensis Syd., in Syd. \& S. Ahmad 1939. - Ustilago panjabensis (Syd.) L. Ling 1950.

Sorosporium penniseti Mundk. 1939a.

Sphacelotheca stewartii Mundk. 1944b.

Ustilago penniseti var. verruculosa Massenot, in Guyot, Malençon \& Massenot 1969.

Sori in all spikelets of an inflorescence, cylindrical, $1-1.5 \times 2-7 \mathrm{~mm}$, hidden by the outer floral envelopes, covered by a greyish brown peridium which flakes away exposing the semi-agglutinated to granular-powdery mass of spore balls and a central columella of the length of the sori, with a few, short, apical branches. Spore balls globose, ovoid to ellipsoidal, 40-110 $\mu \mathrm{m}$ long, dark reddish brown, composed of many spores that separate rather easily. Spores dimorphic. Outer spores globose to ellipsoidal, 9-13 $\times(9-) 10-13.5(-14.5) \mu \mathrm{m}$, medium reddish brown, finely and densely echinulate. Inner spores subpolyhedral, smaller $(7-10 \mu \mathrm{m})$, paler coloured and finely punctate or apparently smooth. Sterile cells of the peridium variable in shape and size, often elongate, 4-16 $\mu \mathrm{m}$ long, arranged in parallel rows, hyaline, smooth. Spore germination results in 2-celled basidia, each cell producing a long, ramifying hypha (Fig. 29).

On Poaceae: Pennisetum orientale Rich., Golestan Prov., Gonbad city, IRAN 4419 F; West Azarbaijan Prov., Khoy city, Ghotur area, IRAN 8234 F.

39. Sporisorium persicum Vánky \& H. Scholz, Nova Hedwigia 72: 397, 2001.

Sori in all spikelets of an inflorescence, destroying the inner floral organs, protruding between the glumes, long ovoid or cylindrical, $1-2 \times 3-8 \mathrm{~mm}$, initially covered by a greyish brown peridium that ruptures irregularly exposing the blackish brown, powdery mass of spore balls, spores and sterile cells surrounding a stout, tapering columella. Spores in loose, many-spored balls, when mature single, subglobose, ellipsoidal or slightly irregular, variable in size, 5-8 $\times 5.5-9 \mu \mathrm{m}$, yellowish brown; wall even, c. $0.5 \mu \mathrm{m}$, finely, moderately densely punctate-verruculose, spore profile smooth. Sterile cells in irregular groups or in chains, individual cells variable in shape and size, subglobose, ellipsoidal or irregular, with one or several flattened contact sides, usually larger than the spores, 5$16 \times 6-17 \mu \mathrm{m}$, hyaline; wall c. $1 \mu \mathrm{m}$ thick, smooth.

On Poaceae: Spodiopogon pogonanthus (Boiss. \& Bal. ex Bal.) Boiss.; Fars Prov., North of Kazeron, Bishapur, 23.IV.2000, W. Lang,

Remarks - Known only from the type collection in Iran.

40. Sporisorium reilianum (J.G. Kühn) Langdon \& Full., Mycotaxon 6: 452, 1978.

Ustilago reiliana J.G. Kühn, in Rabenhorst 1875. - Cintractia reiliana (J.G. Kühn) Clinton 1900. Sphacelotheca reiliana (J.G. Kühn) Clinton 1902. - Sorosporium reilianum (J.G. Kühn) McAlpine 1910.

Ustilago holci-sorghi Rivolta 1873 (nom. dub.). - Sphacelotheca holci-sorghi (Rivolta) Cif. 1938. Sorosporium holci-sorghi (Rivolta) Moesz 1950. - Sporisorium holci-sorghi (Rivolta) Vánky 1985a.

Ustilago reiliana J.G. Kühn f. zeae Pass., in Rabenhorst 1876.

Ustilago pulveracea Cooke 1876.

Ustilago abortifera Speg. 1899.

Sorosporium simii Pole-Evans 1916.

Ustilago reiliana f. sorghi Geschele 1927 (nom. nud.). - Sorosporium holci-sorghi f. sorghi (Geschele) Săvul. 1957 (comb. illegit.).

Sori in inflorescence which are usually completely destroyed and transformed into blackish brown, granular-powdery spore mass, but sometimes only single flowers are infected and rarely the panicle rachis and leaves are infected. Sori initially covered by a white to pale brown peridium of fungal origin which ruptures irregularly and flakes away to expose the mass of spore balls mixed with groups of sterile cells and numerous long, sinuous, thread-like or stout columellae. Spore balls 60$100 \mu \mathrm{m}$ long, composed of numerous, loosely connected spores. Spores globose, subglobose to ovoid or slightly irregular, $10-13 \times 10.5-14.5 \mu \mathrm{m}$, light olive-brown, densely, minutely echinulate. 
Sterile cells in irregular, persistent groups, rounded on the free surface, flattened on the contact surfaces, 8-16 $\mu \mathrm{m}$ long, hyaline to yellow tinted, smooth, with a few oil droplets in each cell. Spore germination results in 4-celled basidia on which lateral and terminal basidiospores are produced (Fig. 30).

On Poaceae: Sorghum bicolor (L.) Moench, East Azarbaijan Prov., Mianeh city; Ardabil Prov., Moghan area; Sistan va -Baluchestan Prov., Chah-Bahar port, Dashtiari area, IRAN 8031 F; Esfahan Prov., Kashanak city, Ardestan city, IRAN 10323 F; Zanjan Prov.

On Sorghum halepense (L.) Pers., Ardabil Prov., Moghan city; Mazandaran Prov., Neka city; Khuzestan Prov., Dezful city; Mazandaran Prov., Tonekabon city, IRAN 4417 F; Khuzestan Prov., Dezful city, Safiabad agricultural station, IRAN 6581 F; Yazd Prov., Marvast city, IRAN 13879 F; Tehran Prov., Varamin city, IRAN 4416 F; Tehran Prov., Karaj city, IRAN 4413 F; Lorestan Prov., Khorramabad city, Alashtar city, IRAN 4414 F; Kermanshah Prov., Gilan-Gharb city, IRAN 6633 F; Fars Prov., Malusjan village, IRAN 13283 F; Fars Prov., Shiraz city, Dinakan village, IRAN 13192 F; West Azarbaijan Prov., Oroumieh city, IRAN 4415 F; Mazandaran Prov., Sari city, Baye Kola village, IRAN 11191 F; Kermanshah Prov., Bisotun area, IRAN 2533 F; Fars Prov., Road of Sepidan, Pagah village, IRAN 13191 F; Esfahan Prov., Semirom city, Bide village, IRAN 11190 F.

On Sorghum vulgare Pers., Mazandaran Prov., Babol city, IRAN 4412 F.

On Zea mays L., Esfahan Prov., Esfahan city, IRAN 4418 F; Ardabil Prov., Moghan area.

Remarks. Khabiri (1958) reported this fungus as Sphacelotheca holci-sorghi on Sorghum saccharatum from unknown locality in Iran.

41. Sporisorium schweinfurthianum (Thüm.) Vánky, Publ. Herb. Univ. Uppsala 11: 12, 1983. Ustilago schweinfurthiana Thüm. 1877. - Sphacelotheca schweinfurthiana (Thüm.) Sacc. 1908. Sporisorium schweinfurthianum (Thüm.) Karatygin, in Karatygin \& Azbukina 1989 (comb. superfl.).

Sori destroying all inner floral organs of an inflorescence, cylindrical, 4-6 mm long, protruding between the glumes, initially covered by a greyish brown peridium that ruptures apically exposing a dark brown, semi-powdery spore mass and a well-developed, central columella. Infection systemic. Spores when young in firmly adhering masses, single at maturity, globose, subglobose, ovoid, often slightly irregularly polyhedral, $10-13 \times 11-14 \mu \mathrm{m}$, olive-brown; wall $0.8-1 \mu \mathrm{m}$ thick, very finely punctate-verruculose. Sterile cells abundant among the spores, in groups or in chains, individual cells variable in size (smaller, the same size or larger than the spores), hyaline, thin-walled, smooth, collapsed in old specimens; those of the peridium 3-20 $\mu \mathrm{m}$ long, usually arranged in parallel chains. Spore germination of Ustilago-type. A spore produces one to several septate basidia which develop either basidiospores or, after conjugation of two basidial cells, hyphae (Fig. 31).

On Poaceae: Imperata cylindrica (L.) P. Beauv., Tehran Prov., Shahriar city, IRAN 8077 F; Khuzestan Prov., between Ahvaz city \& Haft-Tappeh city; Golestan Prov., Aliabad city, IRAN 7127 F; Golestan Prov., Kalaleh city to Maraveh-tappeh city, Gogjeh village, IRAN 11361 F; Golestan Prov., Gonbad city, Galikesh village, IRAN 4408 F; Golestan Prov., Gorgan city, Agh-ghala village, Araghi Mahaleh agriculture station, IRAN 11178 F; Golestan Prov., Gorgan city, IRAN 10841 F; Golestan Prov., Gorgan city, 34 km SW of Maraveh tappeh city, IRAN 7264 F; Khuzestan Prov., Ahvaz city, IRAN 4409 F; Khuzestan Prov., Dezful city, Safiabad agriculture station, IRAN 4410 F; Tehran Prov., Karaj city, IRAN 4407 F; Sistan va -Baluchestan Prov., Saravan city, IRAN 8782 F; East Azarbaijan Prov., Arasbaran protected area, Tatar village toward Asheghloo village, Sotan area, IRAN 14217 F; Qazvin Prov., Takestan city, IRAN 10518 F; Kerman Prov., Rafsanjan city, Ravar village, IRAN $671 \mathrm{~F}$.

42. Sporisorium sorghi Ehrenb. ex Link, Linné's Species Plantarum, Ed. 4, 6(2): 86, 1825.

Sphacelotheca sorghi (Ehrenb. ex Link) G.P. Clinton 1902. - Cintractia sorghi (Ehrenb. ex Link) Hirschh. 1939b.

Ustilago sorghicola Speg. 1902. - Sphacelotheca sorghicola (Speg.) Zundel 1930b. 
Tilletia sorghi-vulgaris Tul. \& C. Tul. 1847. - Cintractia sorghi-vulgaris (Tul. \& C. Tul.) G.P. Clinton 1897. - Ustilago tulasnei J.G. Kühn 1874 (nom. nov. superfl. pro T. sorghi-vulgaris).

Sori in spikelets as 3-10 mm long, ovoid or cylindrical, pale brown bodies protruding from the glumes, covered by a well-developed peridium of interwoven hyphae overlain by host tissue, which ruptures irregularly to expose the dark brown, powdery spore mass and the central columella composed of host tissues permeated by hyphae. The panicles may or not be congested and stunted. Spores when mature single, globose, subglobose, ovoid to slightly irregular, 5.5-7 $\times 5.5-8(-8.5)$ $\mu \mathrm{m}$, light olive-brown, from apparently smooth to sparsely punctate or very finely verruculose. Sterile cells somewhat larger than the spores, in irregular groups or chains, hyaline, smooth. Spore germination results in 4-celled basidia (the 4th, basal cell usually remains in the spore) producing lateral and apical basidiospores in nutrient media, or infection hyphae in water (Fig. 32).

On Poaceae: Sorghum bicolor (L.) Moench, Azarbaijan Prov., IRAN 8589 F; Khuzestan Prov., Dezful city; Tehran Prov., Karaj city; Esfahan Prov., Kashan city; Zanjan Prov., Zanjan city; Esfahan Prov., Najafabad city, IRAN 6601 F; Esfahan Prov., Ardestan, Zavareh city, Aliabad village, IRAN $10321 \mathrm{~F}$.

On Sorghum cernuum Willd., Sisitan va Baluchestan Prov., IRAN 4406 F.

On Sorghum halepense (L.) Pers., Mazandaran Prov., Moghan city, IRAN 7123 F.

On Sorghum sp., Kordestan Prov.

A smut fungus was reported under the name S. sorghi by Sharif \& Ershad (1966) on Zea mays L. from Lorestan Prov., Boroujerd city. Most probably it refers to Sporisorium reilianum.

43. Sporisorium tricholaenae (Henn.) Vánky, Mycotaxon 33: 367, 1988 b. Ustilago tricholaenae Henn. 1893a, b. - Sphacelotheca tricholaenae (Henn.) Mundk. 1944a. Sphacelotheca panici-teneriffae Picb. 1932.

Sori in all flowers of an inflorescence, ovoid to long-cylindrical, $0.5-1 \times 1.5-6 \mathrm{~mm}$, partly hidden by the glumes, initially covered by a greyish brown, thick peridium of fungal and host origin, which later ruptures exposing the blackish brown, semi-agglutinated to powdery mass of spore balls, spores and sterile cells surrounding a central, flagelliform columella, c. the length of the sori. Spore balls subglobose, ovoid, ellipsoidal, elongate or irregular, 30-60 × 30-80(-100) $\mu \mathrm{m}$, dark reddish brown, composed of many, easily separating spores. Spores subglobose, ellipsoidal or subpolyhedrally slightly irregular, $(6.5-) 7-9(-10) \times 7-10(-11) \mu \mathrm{m}$, dark brown; wall even, c. $1 \mu \mathrm{m}$ thick, densely punctate, verruculose to finely echinulate, spore profile smooth to rough. Sterile cells in irregular groups or chains, individual cells variable in shape and size, 5-12 $\mu \mathrm{m}$ long, hyaline, thin-walled. Spore germination results in 4-celled basidia producing laterally and terminally ovoid to elongate basidia or, after conjugation of two basidial cells, dikaryotic hyphae (Fig. 33).

On Poaceae: Tricholaena teneriffae (L. f.) Link, Hormozgan Prov., $15 \mathrm{~km} \mathrm{~N}$ of Bandare Lengeh port, IRAN 8770 F; Sistan va-Baluchestan Prov., Ghasre-Ghand city, IRAN 9249 F.

This smut was reported on Hyparrhenia hirta (L.) Stapf. by Karampour et al. (2008). This plant is not a host of Sporisorium tricholaenae.

44. Sporisorium vanderystii (Henn.) Langd. \& Fullerton, Mycotaxon 6: 451, 1978.

Ustilago vanderystii Henn., in Wildeman 1907. - Cintractia vanderystii (Henn.) Zundel 1930b. —

Sphacelotheca vanderystii (Henn.) L. Ling 1951.

Ustilago hyparrheniae Beeli 1922. — Sphacelotheca hyparrheniae (Beeli) Zambett. 1980('1979').

Sphacelotheca evansii Zundel 1930b.

Sphacelotheca ritchiei Zundel 1930b.

Ustilago puellaris Syd. 1935. - Sporisorium puellare (Syd.) G. Deml, in Vánky, Deml \& Oberwinkler 1988.

Sphacelotheca ruprechtii Syd. 1935.

Sphacelotheca kenyae Zundel 1937.

Ustilago hyparrheniae J.C.F. Hopkins 1938 (later homonym, not Beeli 1922). 
Sori comprising all raceme-pairs or racemes of an inflorescence, cylindrical, usually forked, Y- or V-shaped, 1-2(-2.5) $\times 4-8 \mathrm{~mm}$, partly concealed by the spathae, initially covered by a thick, brown peridium that ruptures irregularly exposing the semi-agglutinated to powdery mass of spores and sterile cells surrounding a stout, bifurcate or a simple columella, c. the length of the sori, often with short branches. Rarely, the sori are restricted to the spikelet-pairs only. Spores when mature single, globose, ovoid, ellipsoidal to rarely elongate or slightly irregular, (6.5-)7-10.5 × (7-)8-11 $\mu \mathrm{m}$, yellowish brown; wall evenly thick, $0.5-0.8 \mu \mathrm{m}$, finely, densely punctate-verruculose, spore profile smooth, in SEM spore surface finely, densely echinulate, between the spines finely, densely verruculose. Sterile cells in small, irregular groups, individual cells globose, ellipsoidal or irregularly polyangular with flattened side(s), 9-24(-30) $\mu \mathrm{m}$ long, hyaline or subhyaline; wall $0.5-1.5 \mu \mathrm{m}$ thick, smooth. Spore germination results in a ramified, multicellular, monokaryotic hypha (Fig. 34).

On Poaceae: Hyparrhenia hirta (L.) Stapf, Kermanshah Prov., Rijab, Sahalan village toward Sarab-e Shalan, alt. 1100 m, 14.V.2006, leg. M. Abbasi, R. Fritsch \& M. Keusgen, IRAN 14783 F, HUV 21826.

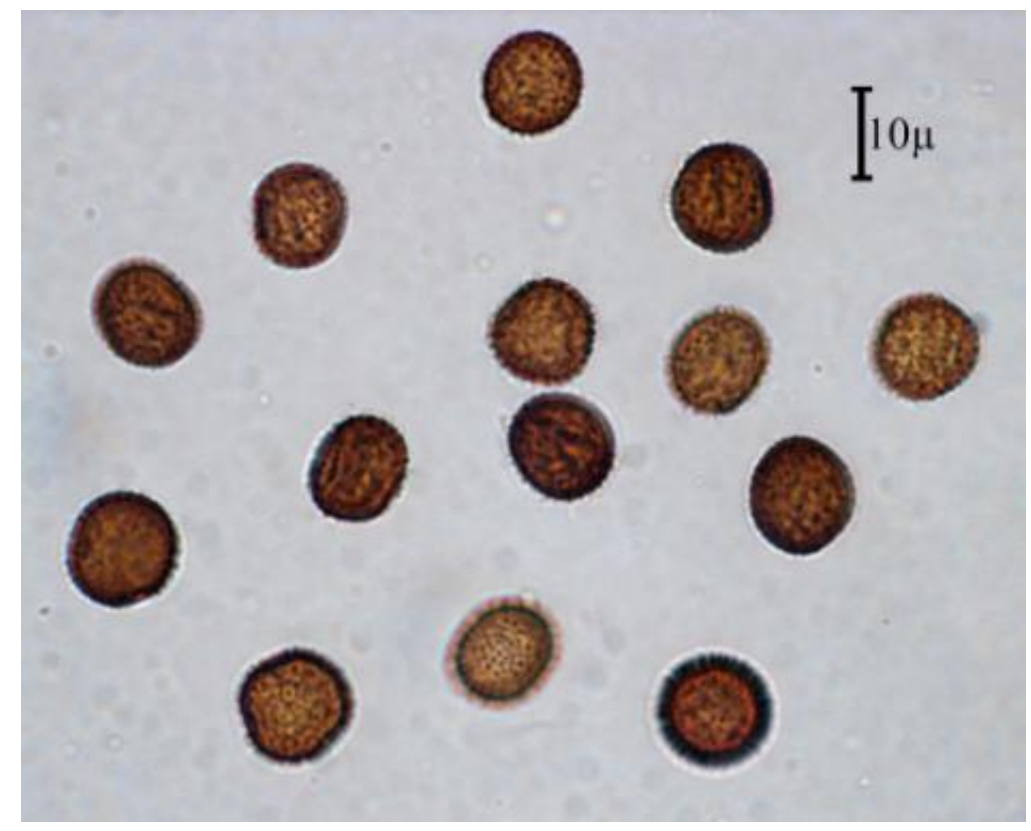

Fig.20 - Sporisorium aegyptiacum on Schismus arabicus (7233)

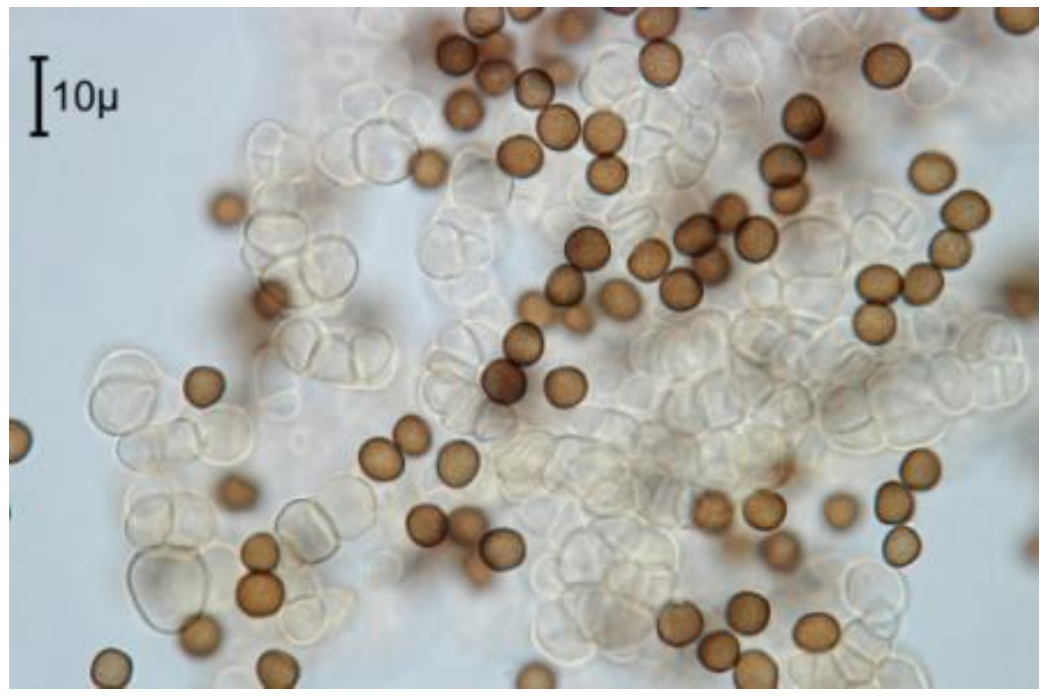

Fig.21 - Sporisorium andropogonis on Dichanthium ischaemum (11020) 

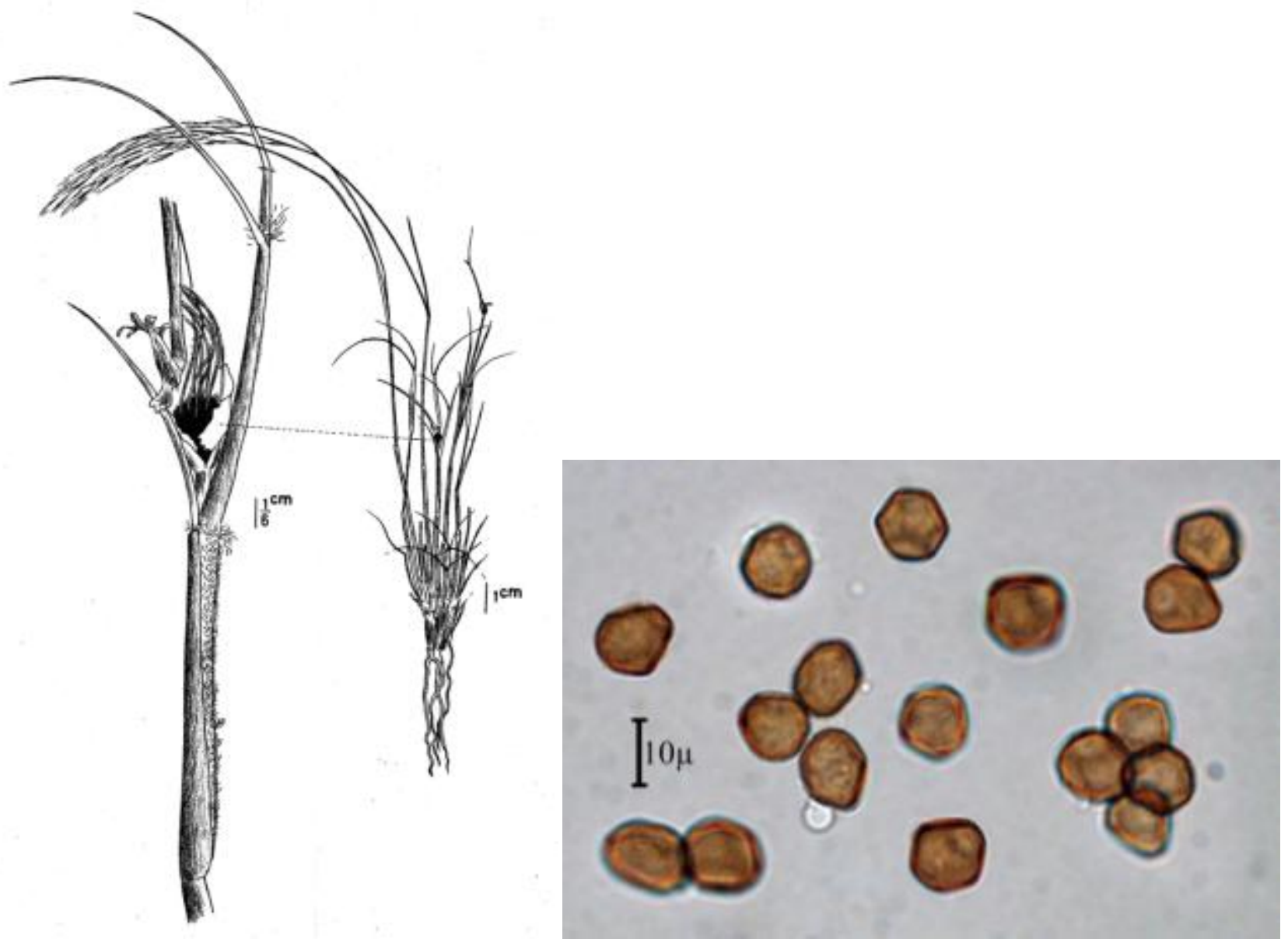

Fig.22 - Sporisorium aristidae-lanuginosae on Stipagrostis plumosa, infected plant (14881) (left) and teliospores (8300) (right)

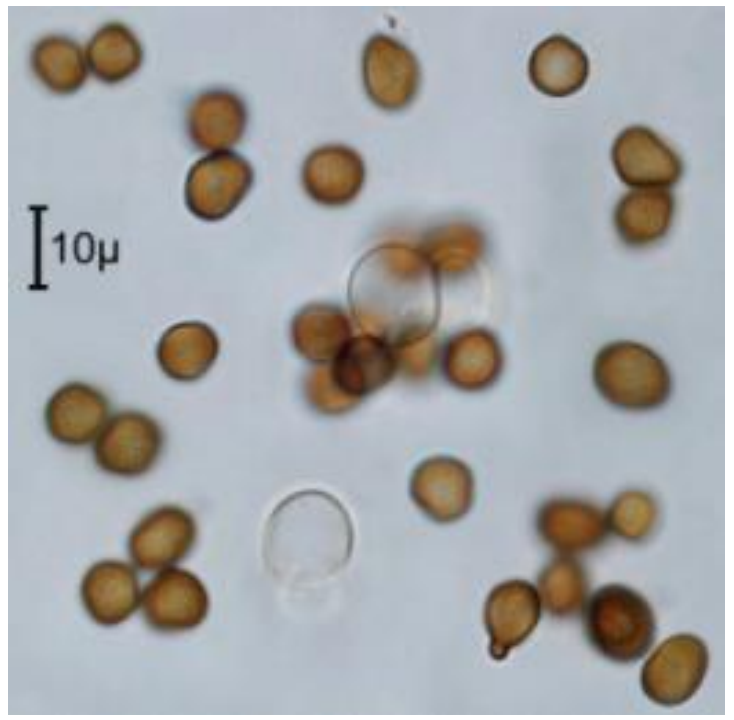

Fig.23 - Sporisorium cruentum on Sorghum halepense (9563) 


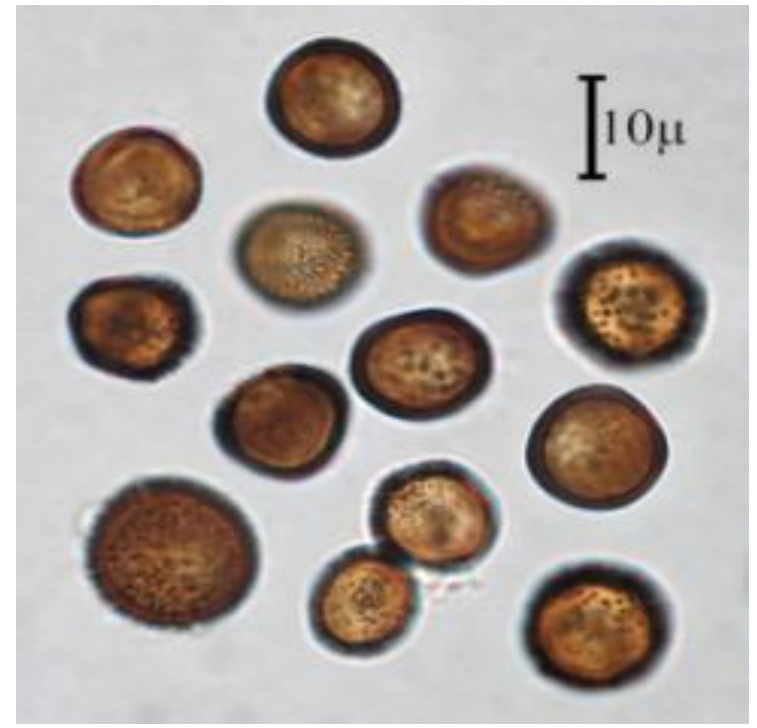

Fig.24 - Sporisorium desertorum on Lasiurus sindicus (11288)

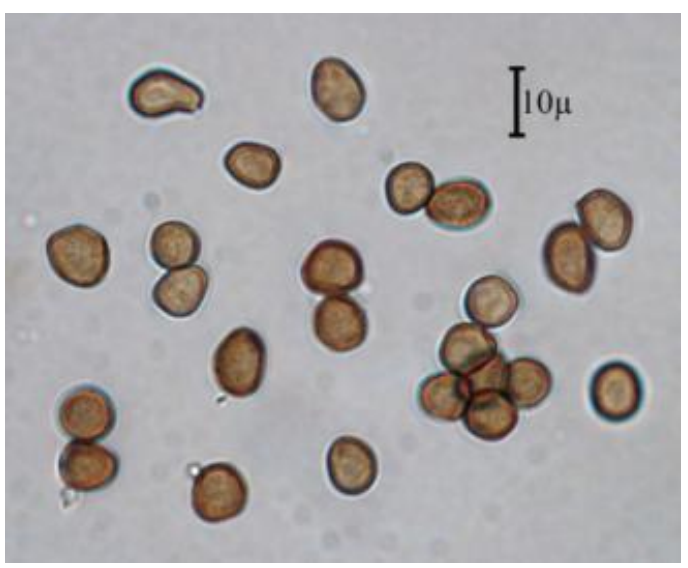

Fig.25 - Sporisorium destruens on Panicum miliaceum (10320)

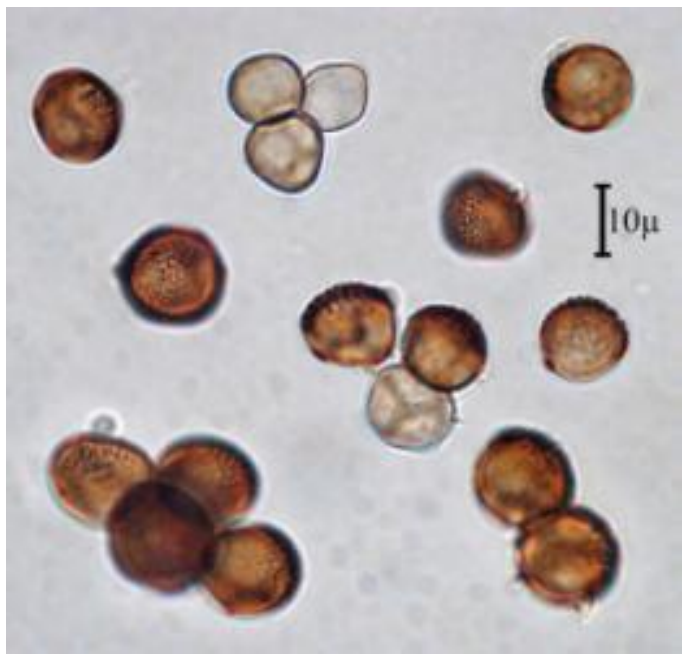

Fig.26 - Sporisorium ehrenbergii on Sorghum bicolour (10322) 


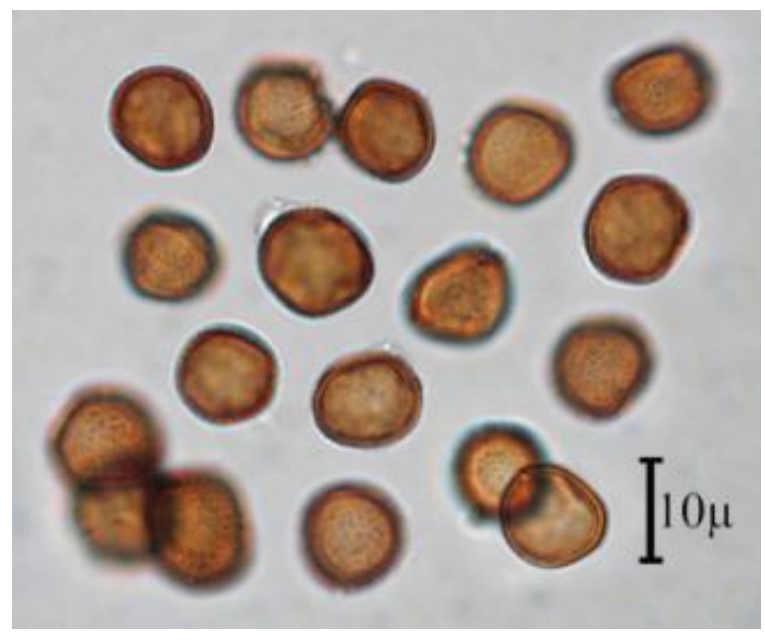

Fig.27 - Sporisorium foveolati on Dichanthium foveolatum (4425)

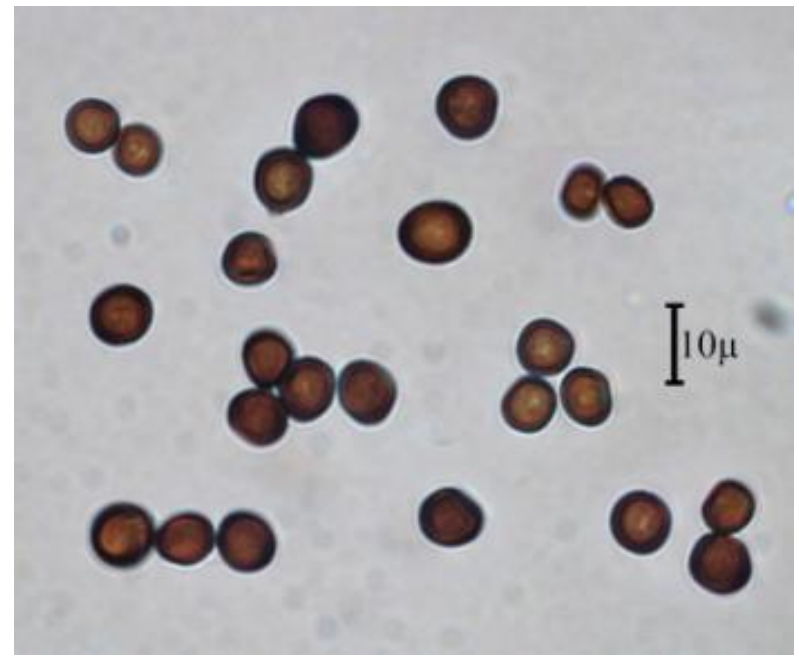

Fig.28 - Sporisorium lanigeri on Cymbopogon olivieri (12399)

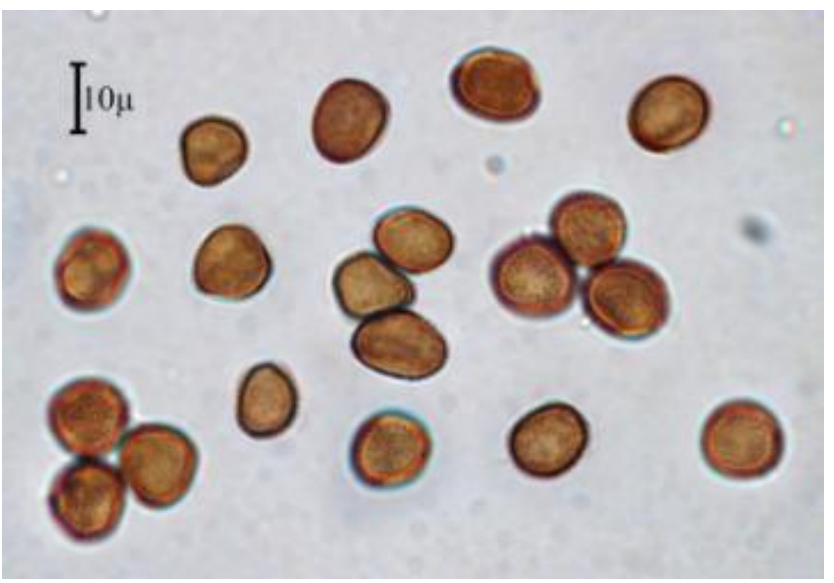

Fig.29 - Sporisorium penniseti on Pennisetum orientale (4419) 


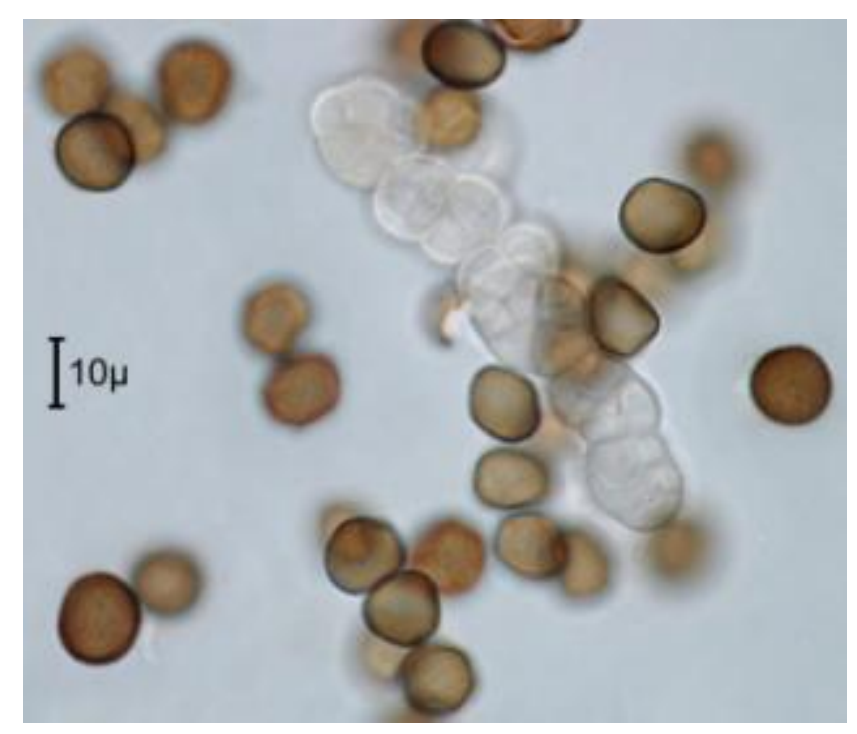

Fig.30 - Sporisorium reilianum on Sorghum halepense (13283)

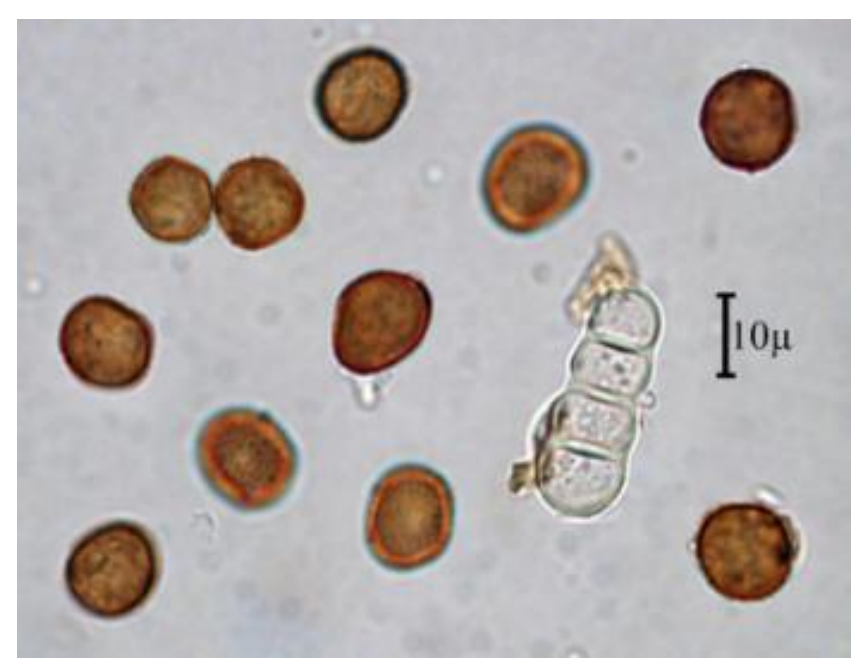

Fig.31 - Sporisorium schweinfurthianum on Imperata cylindrical (14217)

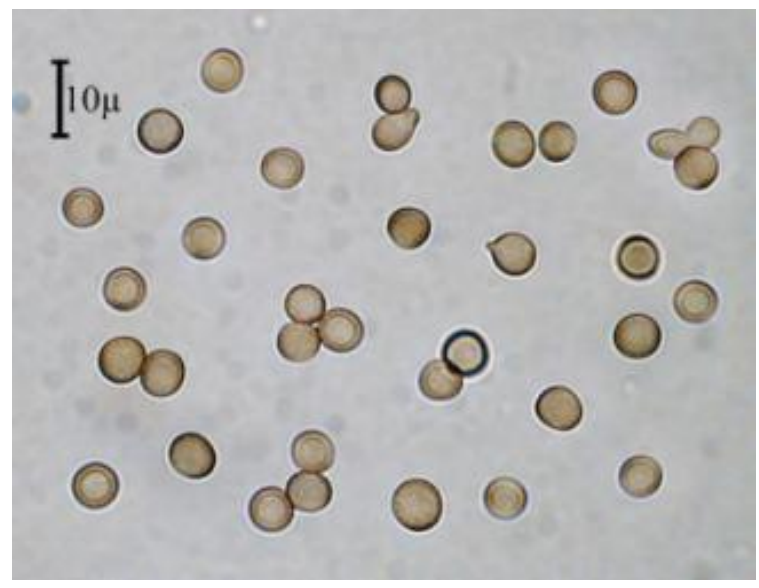

Fig.32 - Sporisorium sorghi on Sorghum bicolour (10321) 


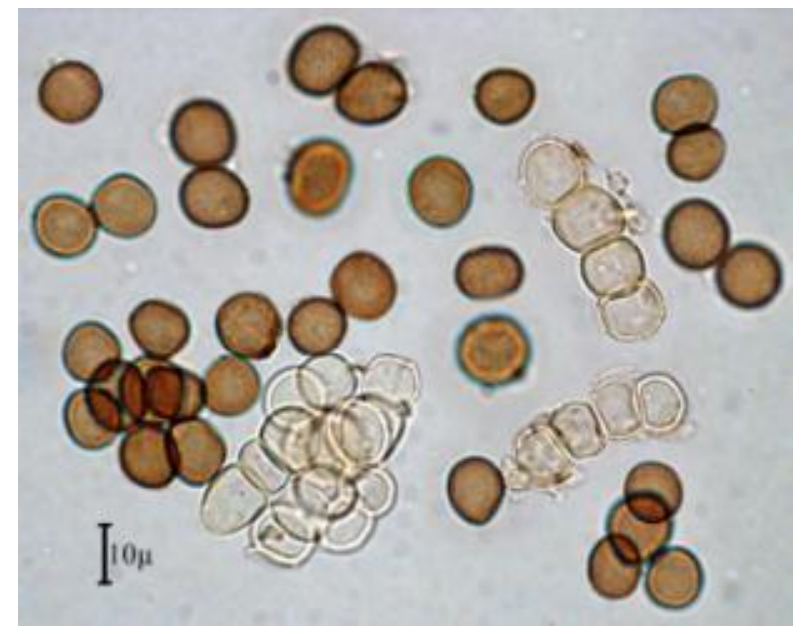

Fig.33 - Sporisorium tricholaenae on Tricholaena teneriffae (9249)

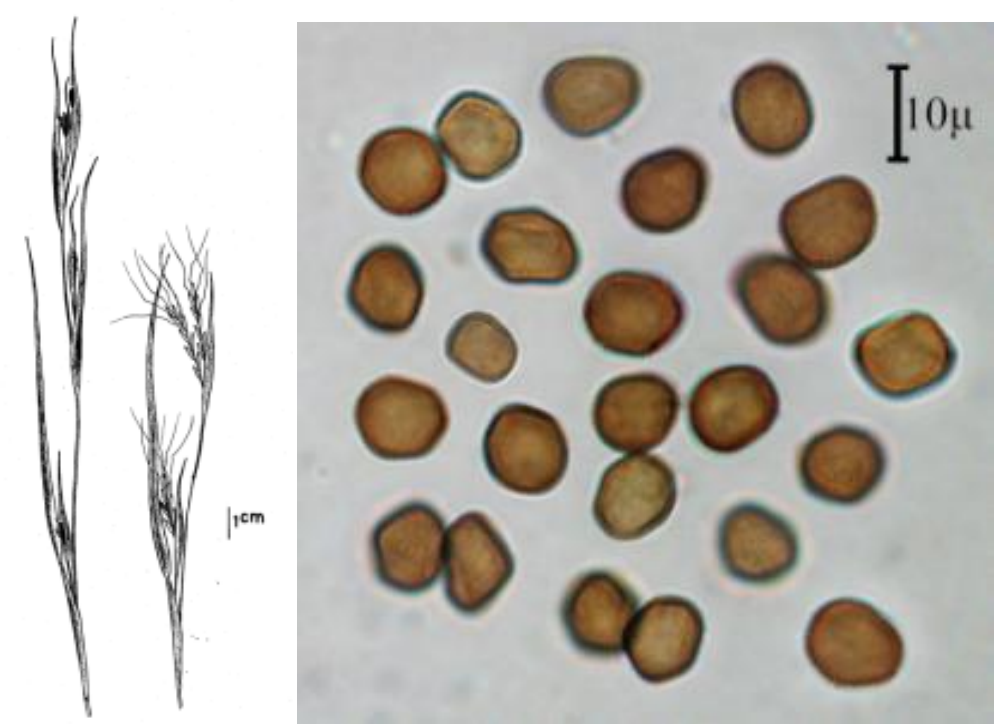

Fig.34 - Sporisorium vanderystii on infected plant (14783) (left) and teliospores (14783) (right)

Tilletia Tul. \& C. Tul.

\section{Key to the Tilletia species based on host plant taxonomy}

1. On Aegilops ....

- Not on Aegilops

2. Host plants more or less dwarfed; muri $1.5-3 \mu \mathrm{m}$ high

T. contraversa

- Host plants not dwarfed; muri 0.5-1.5 $\mu \mathrm{m}$ high. T. caries

3. On Critesion

T. hordei

- Not on Critesion .4

4. On Elymus

T. contraversa

- Not on Elymus .5

5. On Hordeum

T. contraversa

- Not on Hordeum 6

6. On Loliolum T. lolioli

- Not on Loliolum 7 
7. On Lolium

- Not on Lolium ...8

8. On Psathyrostachys

T. hordei

- Not on Psathyrostachys .9

9. On Rostraria T. rostrariae

- Not on Rostraria. 10

10. On Taeniatherum T. bornmuelleri

- Not on Taeniatherum 11

11. On Triticum; spores smooth T. laevis

- On Triticum Spores ornamented. 12

12. Spores provided with blunt warts T. indica

- Spores reticulate .13

13. Host plants more or less dwarfed; muri 1.5-3 $\mu \mathrm{m}$ high

- Host plants not dwarfed; muri 0.5-1.5 $\mu \mathrm{m}$ high.

T. contraversa T. caries

45. Tilletia bornmuelleri Magnus, Bull. Herb. Boissier 3: 574, 1903.

Sori in ovaries, swollen, ovoid to elongate, partly hidden by the glumes, initially greenish, later dark brown containing the indurate to semi-powdery, coppery brown mass of spores. Spores globose, ovoid to slightly subpolyhedrally irregular, 18-24×19-26 $\mu \mathrm{m}$, light to medium reddish brown; wall reticulate, 6-9 meshes per spore diam., reticulum often incomplete to cerebriform, muri 1.5-2 $\mu \mathrm{m}$ high, 27-36 on the spore circumference. Sterile cells globose to ellipsoidal, smaller than the spores (13-20 $\mu \mathrm{m}$ long), subhyaline to pale yellow, contents finely granular; wall 1-2 $\mu \mathrm{m}$ thick, smooth (Fig. 35).

On Poaceae: Taeniatherum asperum (Simonk.) Nevski, Tehran Prov., Karaj-Calus road, Dizin road, near Velayat rood, road side, IRAN 10907 F.

46. Tilletia caries (DC.) Tul. \& C. Tul., Ann. Sci. Nat. Bot., Sér. 3, 7: 113, 1847.

Uredo caries DC. 1815.

Lycoperdon tritici Bjerk. 1775 (nom. nud.). - Tilletia tritici (Bjerk.) Wolff 1874. - Tilletia tritici (Bjerk.) Winter, in Rabenh. 1881 (comb. superfl.).

Uredo sitophila Ditmar, in Sturm 1816. - Caeoma sitophilum (Ditmar) Link 1825. - Tilletia sitophila (Ditmar) J. Schröt., in Cohn 1877.

Sori filling the ovaries with a reddish brown to dark brown, semi-agglutinated to pulverulent, foetid spore mass. Spores globose to subglobose, $(14-) 16-20(-25) \mu \mathrm{m}$ in diam., pale yellow to reddish brown; wall reticulate, 5-8 meshes per spore diam., 2.5-6 $\mu \mathrm{m}$ wide, muri $0.5-1.5 \mu \mathrm{m}$ high, 19-28 on the spore circumference. Sterile cells globose to subglobose, 10-18 $\mu \mathrm{m}$ in diam., hyaline to subhyaline, smooth, thin-walled $(0.5-1.5 \mu \mathrm{m})$. Spore germination results in aseptate basidium on which 8-16, elongate basidiospores are produced apically. These fuse to produce dikaryotic hyphae which may directly infect host seedling coleoptiles or produce further ballistospores (secondary sporidia) (Fig. 36).

On Poaceae: Aegilops triuncialis (L.) Á. Löve, Ardabil Prov., Khalkhal city, Zinab village, IRAN 9848 F; East Azarbaijan Prov., Marand city; West Azarbaijan Prov., Orumieh-Salmas road, IRAN $8211 \mathrm{~F}$.

On Triticum aestivum L., Esfahan Prov.; Khorasan Prov.; Lorestan Prov.; Zanjan Prov.; Ardebil Prov., Ardebil city, IRAN 8588 F; Ilam Prov., Dare-shahr (Abbas-abad) city, IRAN 10297 F; Tehran Prov., Damavand city, Ahmadabad village, IRAN 11587 F; Khuzestan Prov.; East Azarbaijan Prov., Maragheh city; Hamedan Prov.; Gilan Prov.

On Triticum sp., Khuzestan Prov., Ahvaz; Tehran Prov., Karaj. 
Tilletia caries $\boldsymbol{x}$ contraversa (T. intermedia Gassner) on Triticum aestivum, Kohgiluyeh va Buirahmad Prov., Kordlaghari village, IRAN 7287 F.

Remarks - Hybridisation of Tilletia species on wheat is not uncommon. This is most evident in case of a hybrid between the smooth-spored T. levis with the reticulate-spored T. caries, described also as a separate species: Tilletia triticoides T. Săvulescu.

47. Tilletia contraversa J.G. Kühn, in Rabenh., Fgi. eur. no. 1896, 1874; Hedwigia 13: 188, 1874.

Uredo segetum Pers. f. tritici-repentis DC., in Poiret 1808 (nom. nud.). - Tilletia tritici-repentis (DC.) Liro 1938 (comb. illegit.).

Tilletia calospora Pass. 1876.

Tilletia pancicii Bubák \& Ranoj., in Bubák 1909.

Tilletia contraversa var. elymi Zaprom. 1926.

Tilletia elymicola Lavrov 1937.

Tilletia tritici [subsp.] nanifica F. Wagner 1950 (nom. inval.). - Tilletia nanifica (F. Wagner) Săvul. 1956 (comb. illegit.).

Tilletia contraversa Kühn var. prostrata Lavrov 1937. - Tilletia prostrata (Lavrov) Lavrov 1951.

Tilletia aegilopsidis Golovin 1952.

Tilletia brevifaciens G.W. Fisch. 1952.

Sori in swollen ovaries filled with semi-agglutinated to powdery, dark reddish to blackish brown, foetid spore mass. Infection systemic, usually dwarfing the host ("dwarf bunt"). Spores globose to subglobose, 17-21 × 18-23 $\mu \mathrm{m}$, yellowish to reddish brown; wall deeply reticulate, $(4-) 5-6(-7)$ meshes per spore diam., areolae polygonal, usually rather large, regular (occasionally some spores have irregular to cerebriform ornamentation), 3-5 $\mu \mathrm{m}$ wide, muri 1.5-3 $\mu \mathrm{m}$ high, $15-21$ on the spore circumference; a hyaline, gelatinous sheath present. Sterile cells globose or subglobose, 9-22 $\mu \mathrm{m}$ in diam., hyaline, smooth. Spore germination of typical Tilletia-type, at optimum of 3-8 $\mathrm{C}^{\circ}$, stimulated by light (Fig. 37).

On Poaceae: Aegilops triuncialis (L.) Á. Löve, Ardebil Prov., Khalkal city.

On Critesion bulbosum (L.) Á. Löve (Hordeum bulbosum L.). Reported by Esfandiari (1948) from unknown locality.

On Elymus hispidus (Opiz) Melderis (Agropyron intermedium (Host) P. Beauv.), Tehran Prov., Damavand; Zanjan Prov.

On Elymus hispidus subsp. barbulatus (Schur) Melderis (Agropyron intermedium subsp. trichophorum (Link) Asch. \& Graebner; A. trichophorum (Link) K. Richt.). Mentioned by Durán \& Fischer (1961:50) without locality.

On Hordeum vulgare L., Ghom Prov.; Tehran Prov., Tehran.

On Triticum aestivum L., Chaharmahal va Bakhtiari Prov.; Esfahan Prov., Faridan city; Esfahan Prov., Fereidunshahr city; Hamadan Prov., Hamadan; Kordistan Prov., IRAN 6702 F; Lorestan Prov.; Markazi Prov., Sarband area; Zanjan Prov.; Golestan Prov., Kalpoush plain, IRAN 10263 F; West Azarbaijan Prov., Oroumieh city, Heydarlou village, IRAN 8348 F.

48. Tilletia hordei Körn., Hedwigia 16: 30, 1877a.

Tilletia trabutii Jacz. 1893.

Tilletia hordeina Ranoj. 1914.

Sori in swollen ovaries filled with semi-agglutinated to powdery, dark reddish brown, foetid spore mass. Infection systemic, usually dwarfing the host. Spores globose, subglobose to broadly ellipsoidal, 19-22.5 × 19-23 $\mu \mathrm{m}$, yellowish brown; wall deeply reticulate, (3-)4-5(-6) meshes per spore diam., areolae polygonal, usually rather large, regular (occasionally some spores have irregular to cerebriform ornamentation), 3-5 $\mu \mathrm{m}$ wide, muri 1.5-2 $\mu \mathrm{m}$ high, subacute to acute spiniform, 16-24 on the spore circumference, a hyaline, gelatinous sheath present. Sterile cells 
globose to ellipsoidal, smaller than the spores, 9-16 × 10-16 $\mu \mathrm{m}$, subhyaline; wall 1-3 $\mu \mathrm{m}$ thick, smooth (Fig. 38).

On Poaceae: Critesion marinum (Huds.) Á. Löve (Hordeum marinum Huds.; H. maritimum Stokes), Tehran Prov., Karaj-Chalus road, Kalha village, IRAN 10990 F.

On Critesion murinum (L.) Á. Löve subsp. glaucum (Steud.) W.A. Weber (Hordeum murinum subsp. glaucum (Steud.) Tzvelev; H. glaucum Steud.), West Azarbaijan Prov., Oshnavieh city, Khoroshow valley, IRAN 8086 F; Golestan Prov., Gorgan city, 6 km S of Moraveh tappeh village, IRAN 7261 F; Golestan Prov., Gonbad city, IRAN 4402 F.

On Critesion murinum subsp. leporinum (Link) Á. Löve (Hordeum murinum subsp. leporinum (Link) Arcangeli; H. leporinum Link), Golestan Prov., Gonabad city; Golestan Prov., Gorgan city.

Psathyrostachys fragilis (Boiss.) Nevski (Hordeum fragile Boiss.), Kordestan Prov., Pir Omar Kudrun Mt.

Remarks. Pascoe et al. (2005) used molecular phylogenetic and morphological criteria to show that the smut on Critesion in Australia is not the same as Tilletia contraversa. In Vánky (2011'2012':976) the correct name of T. trabutii Jacz. 1893 is T. hordei Körn.

The lectotype of T. hordei is on Hordeum (design. by Lindeberg 1959:70) fragile Boiss. (= Psathyrostachys fragilis (Boiss.) Nevski), Persia (= Iran), Mt. Pir Omar Gudrun [at present in Iraq, $30 \mathrm{~km} \mathrm{NW}$ of Sulaymaniyah, c. $\left.35^{\circ} 46^{\prime} \mathrm{N}, 45^{\circ} 14^{\prime} \mathrm{E}\right]$, alt. c. $1500 \mathrm{~m}$, leg. H.K. Haussknecht, isolectotype HUV 7229!

49. Tilletia indica Mitra, Ann. Appl. Biol. 18: 178, 1931.

Neovossia indica (Mitra) Mundk. 1940.

Sori in some, often only partly destroyed seeds of a spike, concealed by the glumes. Spore mass blackish brown, powdery, held together by the pericarp. Spores globose, subglobose, ovoid to ellipsoidal, 28-40 $(-45) \times 28-47 \mu \mathrm{m}$, yellowish to dark reddish brown, provided with $1.5-6 \mu \mathrm{m}$ high, irregular, blunt warts, 9-17 per spore diam., often 2-4 warts are confluent into irregular groups, 3252 on the spore circumference. Spores produced at the tip of sporogenous hyphae, their remnants on the spores appear sometimes as a hyaline papilla or a narrow appendage. Sterile cells globose, ellipsoidal, sometimes lacrymiform or irregular, variable in size, 8-28 $\times 10-40 \mu \mathrm{m}$, from subhyaline to pale yellowish brown; wall 1.5-4.5 $\mu \mathrm{m}$ thick, homogeneous or multilayered, smooth. Intermediate cells common. Spore germination results in holobasidia apically producing numerous, filiform basidiospores on which secondary sporidia are produced. These germinate developing infection hyphae which penetrate through the stomata producing local, floral infection (Fig. 39).

On Poaceae: Triticum aestivum L., Bushehr Prov., Bushehr; Kerman Prov., Jiroft city; Sistan va -Baluchestan Prov., Bampoor city towards Iranshahr city, IRAN 9539 F; Fars Prov., Lar city, IRAN 9454 F; Fars Prov., IRAN 10266 F.

Remarks. Known as partial or Karnal bunt of wheat.

50. Tilletia laevis J.G. Kühn, in Rabenh. Fgi. eur. no. 1697, 1873; Hedwigia 12: 152, 1873.

Erysibe foetida Wallr. 1833 (nom. nov. illegit. pro Caeoma sitophilum (Ditmar) Link, et pro Uredo caries DC., i.e., Tilletia caries). - Tilletia foetida (Wallr.) Liro 1920 (comb. illegit.).

Ustilago foetens Berk. \& M.A. Curtis, in Berk. 1874. - Tilletia foetens (Berk. \& M.A. Curtis) J. Schröt., in Cohn 1877.

Sori in ovaries. Spore mass powdery, olive-brown, foetid. Spores globose, ovoid to irregular, 14-17 $\times 16-24 \mu \mathrm{m}$, pale yellowish to olive-brown, contents granular, guttulate or vacuolate; wall 1-1.5 $\mu \mathrm{m}$ thick, smooth, often with a short papilla or mycelial fragment. Sterile cells sparse to abundant, hyaline, globose to irregular, $11-18 \mu \mathrm{m}$ in diam.; wall smooth, thin $(0.5-1.2 \mu \mathrm{m})$. Spore germination of Tilletia-type. Spores attach to the grains at harvest, and then germinate in moist soil. Compatible basidiospores fuse and the resulting dikaryotic hyphae infect coleoptiles of seedlings (Fig. 40).

On Poaceae: Triticum aestivum L. (Triticum vulgare Vill.). Common in Iran. 
51. Tilletia lolii Auersw. ex G. Winter, Rabenh. Krypt.-Fl., 2 Aufl., 1(1): 109, 1881.

Tilletia lolii Auersw., in Rabenh. 1855 (nom. nud.); 1855 (nom. nud.).

Sori in all ovaries of an inflorescence, covered by the pericarp, appearing between the glumes as light to dark brown bodies, 2-4 mm in diam. Spore mass light cinnamon brown to dark brown, semiagglutinated to pulverulent, foetid. Infection systemic at seedling stage. Spores globose to subglobose, less frequently ovoid, 17.5-22 × 17.5-24 $\mu \mathrm{m}$, pale yellow to pale yellowish brown; wall reticulate, rarely incompletely, (5-)6-8 meshes per spore diam., muri 1.5-3 $\mu \mathrm{m}$ high, spiniform with acute or subacute tip, 25-31 on the spore circumference, sheath absent. Sterile cells globose to ovoid, 13-19 $\mu \mathrm{m}$ long, pale yellow, contents granular; wall 1-2 $\mu \mathrm{m}$ thick, smooth. Spore germination results in holobasidia with a dichotomous apex on which 8-12, cylindrical, conjugating basidiospores are produced that develop ballistospores (Fig. 41).

On Poaceae: Lolium rigidum Gaudin, Golestan Prov., Gorgan city, $17 \mathrm{~km} \mathrm{~S}$ of Moraveh tappeh village, IRAN 7257 F; Gilan Prov., N. shore of Lake White River dam, IRAN 7258 F.

52. Tilletia lolioli Vánky, Carris, Castl. \& H. Scholz, in Vánky, Mycotaxon 91: 254, 2005.

Sori in all ovaries of an infected plant, visible between the spreading floral envelopes as ovoid or ellipsoidal, blackish brown bodies, $0.5-0.8 \times 1-1.5 \mathrm{~mm}$, initially covered by the thin pericarp which ruptures irregularly at maturity exposing the blackish brown, powdery mass of spores and sterile cells. Spores globose, subglobose to ellipsoidal, 20-24 × 21-27 $\mu \mathrm{m}$, yellowish to chestnut brown, reticulate, meshes polyangular, variable in size and form, $6-10$ per spore diam., muri 1-1.5 $\mu \mathrm{m}$ high, in optical median view acute, subacute or blunt, 30-39 on the spore circumference, embedded in a thin, hyaline, gelatinous layer. Sterile cells subglobose, ellipsoidal, ovoid or irregular, 9.5-15 × 12$18 \mu \mathrm{m}$, hyaline; wall 0.5-1 $\mu \mathrm{m}$ thick, smooth (Fig. 42).

On Poaceae: Loliolum subulatum (Banks \& Sol.) Eig (Nardurus subulatus (Banks \& Sol.) Bor), Tehran Prov., Karaj city, Dashte Mt., IRAN 10548 F; Gilan Prov., c. 85 km S of Rasht city, East end of lake White River dam, $10 \mathrm{~km}$ NW of Lushan city, IRAN $7259 \mathrm{~F}$.

Remarks. Tilletia lolioli differs from $T$. bromi especially in having darker spores, more meshes per spore diam., lower and more muri on the spore circumference.

53. Tilletia rostrariae Vánky \& Ershad, Rostaniha 3: 20, 2002.

Sori in all ovaries of an inflorescence, ovoid, with a short, acute tip, $0.5-0.8 \times 1-1.5 \mathrm{~mm}$, hidden by the floral envelopes and covered by a thin, dark brown peridium (pericarp) which ruptures at maturity exposing the blackish brown, powdery mass of spores mixed with sterile cells. Spores globose, subglobose to rarely broadly ellipsoidal, $(23-) 24-28 \times 24-28(-29.5) \mu \mathrm{m}$, pale to dark smoky brown; wall 2.5-4 $\mu \mathrm{m}$ thick, completely, rarely incompletely reticulate, very rarely cerebriform, (7-)8-13(-14) meshes per spore diam., muri (0.8-)1-2.5 $\mu \mathrm{m}$ high, 27-37 on the spore circumference. Sterile cells globose, ovoid, ellipsoidal to slightly irregular, 14-17 × 15-21 $\mu \mathrm{m}$, hyaline, contents homogeneous; wall c. $1.5 \mu \mathrm{m}$ thick, smooth. Intermediate cells variable in size, colour and ornamentation, 16-36 $\mu \mathrm{m}$ long, from subhyaline to pale smoky brown, from almost smooth, finely verrucose to reticulate or often cerebriform; wall 1-5 $\mu \mathrm{m}$ thick (Fig. 43).

On Poaceae: Rostraria cristata (L.) Tzvelev (Lophochloa phleoides (Vill.) Rchb.), Golestan Prov., Gorgan city, 6 km S of Maraveh-tappeh village, HUV 14898, IRAN 7260 F.

\section{Tracya Syd. \& P. Syd.}

54. Tracya hydrocharidis Lagerh. in Vestergren, Bot. Not. 1902: 175, 1902 (as 'hydrocharitis'). Doassansia reukaufii Henn. 1904.

Sori in leaves and petioles as whitish pale yellow to pale brown, rounded spots with indistinct margins, from a few $\mathrm{mm}$ to several $\mathrm{cm}$ in diam., sometimes coalescing to cover whole leaves, with 
scattered or gregarious spore balls in the host tissue initially as white, later brown, minute, hypophyllous dots. Spore balls globose, ovoid to slightly irregular, 130-260 $\mu \mathrm{m}$ long, composed of numerous, firmly united spores arranged in a single layer on the surface, and a central network of filaments connected with the spores. Spores radially elongate, more or less prismatic, $13-16 \mu \mathrm{m}$ long, yellowish brown, in surface view spores irregularly polygonal, $(5.5-) 7-10(-12) \mu \mathrm{m}$ in diam, contents finely granular; wall finely and moderately densely verruculose on the free, outer surface, on contact surfaces smooth, $0.8-1 \mu \mathrm{m}$ thick, thickest at the base (up to $2.5 \mu \mathrm{m}$ ). Central network composed of pale brown, branched, septate, 1.2-1.5 $\mu \mathrm{m}$ thick, hardened hyphae. Spore germination of Tilletia-type. Anamorph (Savulescuella hydrocharidis Cif.) reported but probably refers to basidiospores of in situ germinated spores.

On Hydrocharitaceae: Hydrocharis morsus-ranae L., Gilan Prov., Bandar-Anzali port, Anzali lagoon, HUV 14901, IRAN 7262 F.

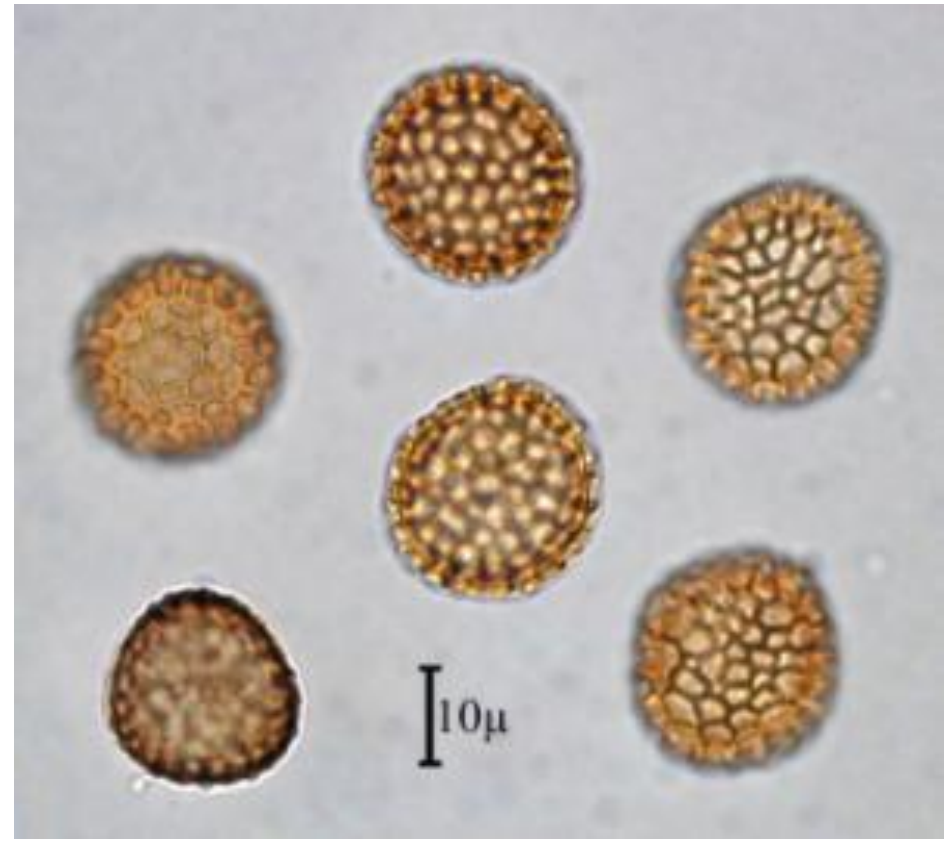

Fig.35 - Tilletia bornmuelleri on Taeniatherum asperum (10907)

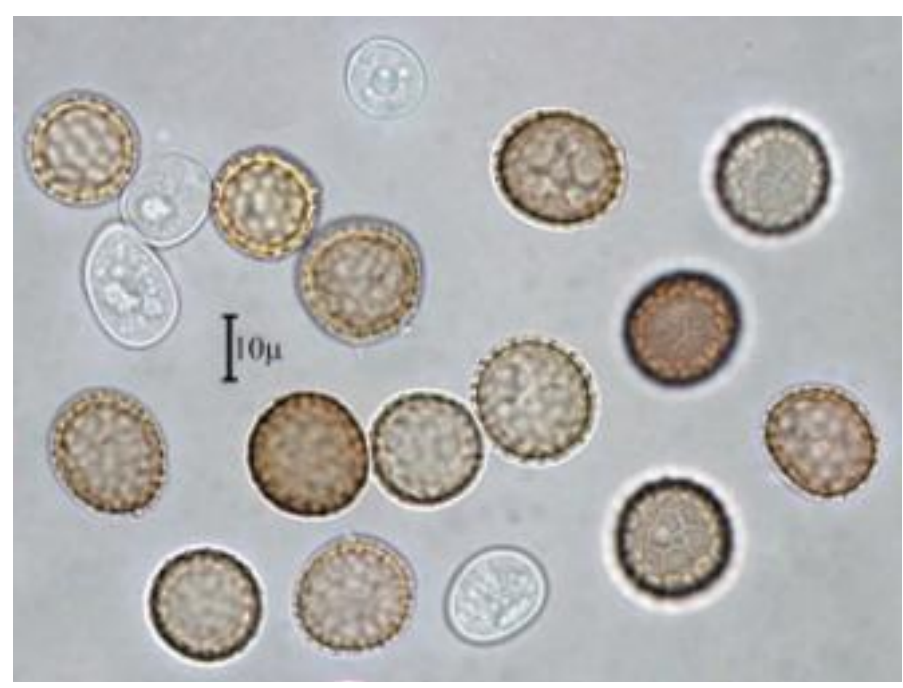

Fig.36 - Tilletia caries on Triticum aestivum (10297) 


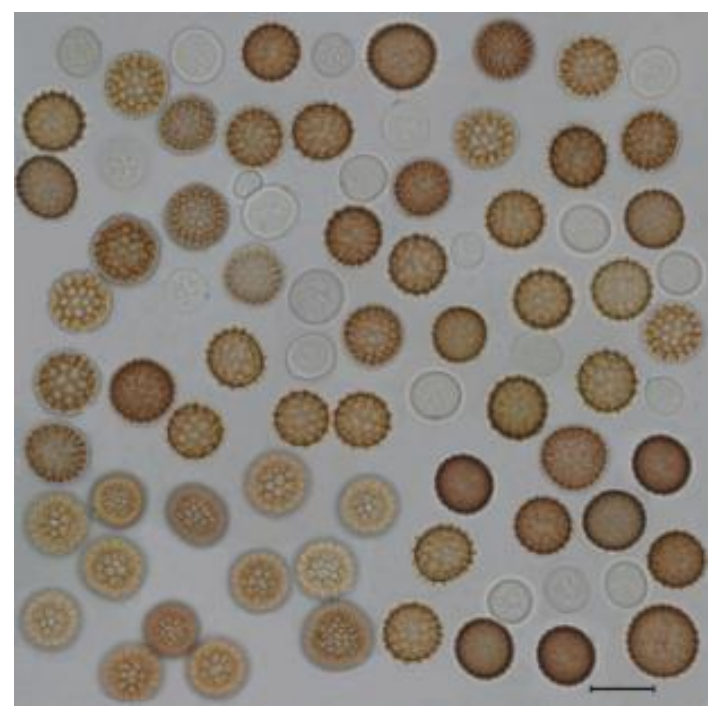

Fig.37 - Tilletia contraversa on Triticum aestivum (8348), Bar=20 $\mu \mathrm{m}$.

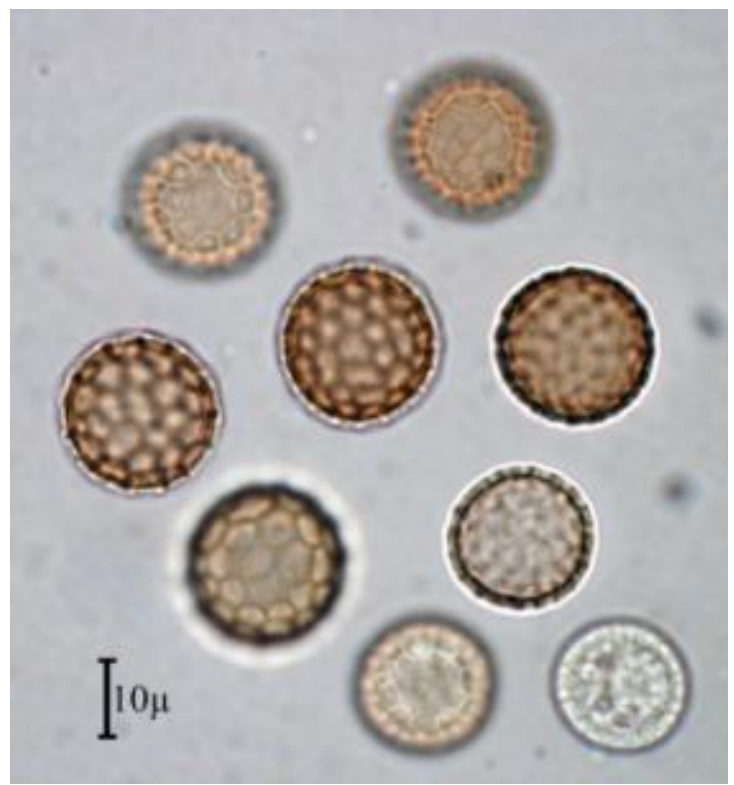

Fig.38 - Tilletia hordei on Critesion murinum subsp. glaucum (4402)

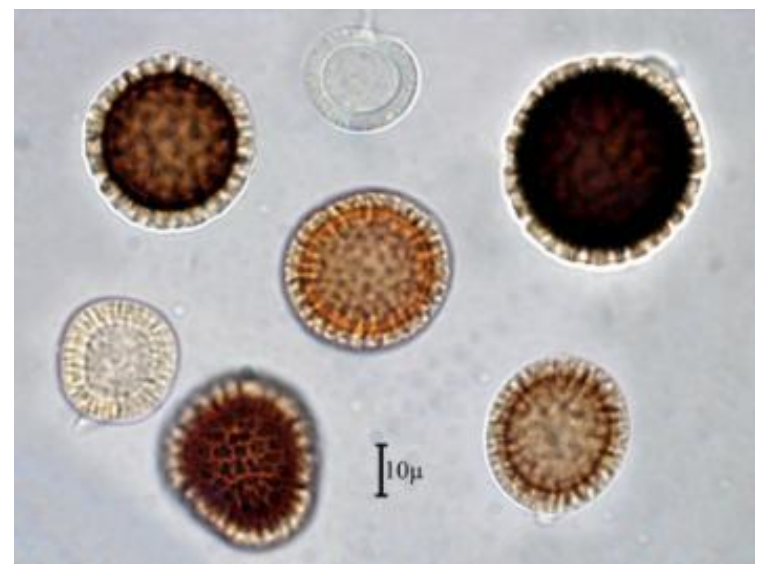

Fig.39 - Tilletia indica on Triticum aestivum (10266) 


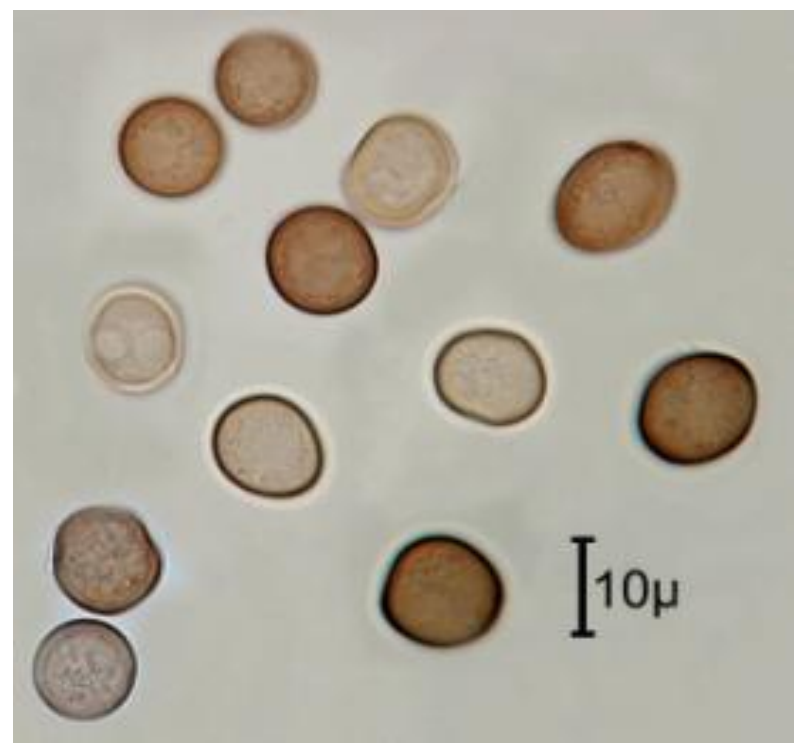

Fig.40 - Tilletia laevis on Triticum aestivum (7221)

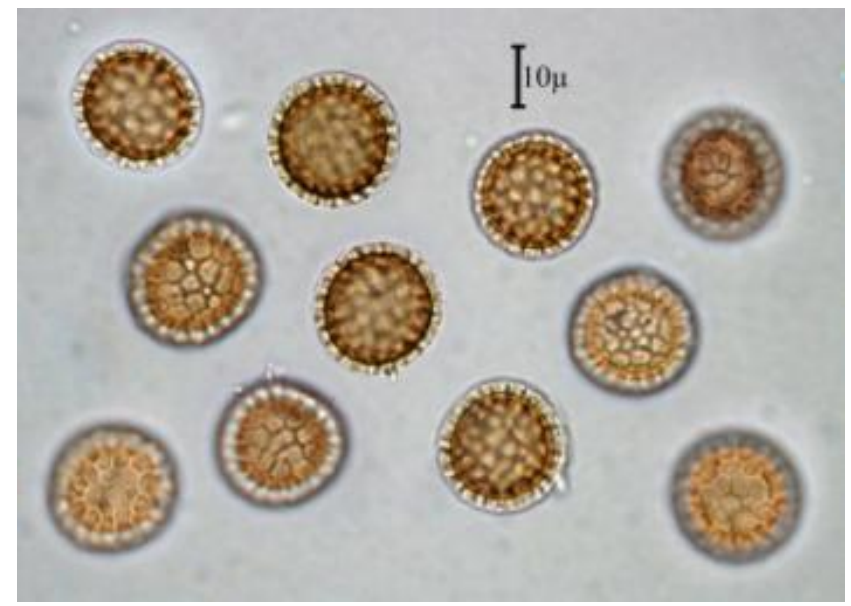

Fig.41 - Tilletia lolii on Lolium rigidum (7257)

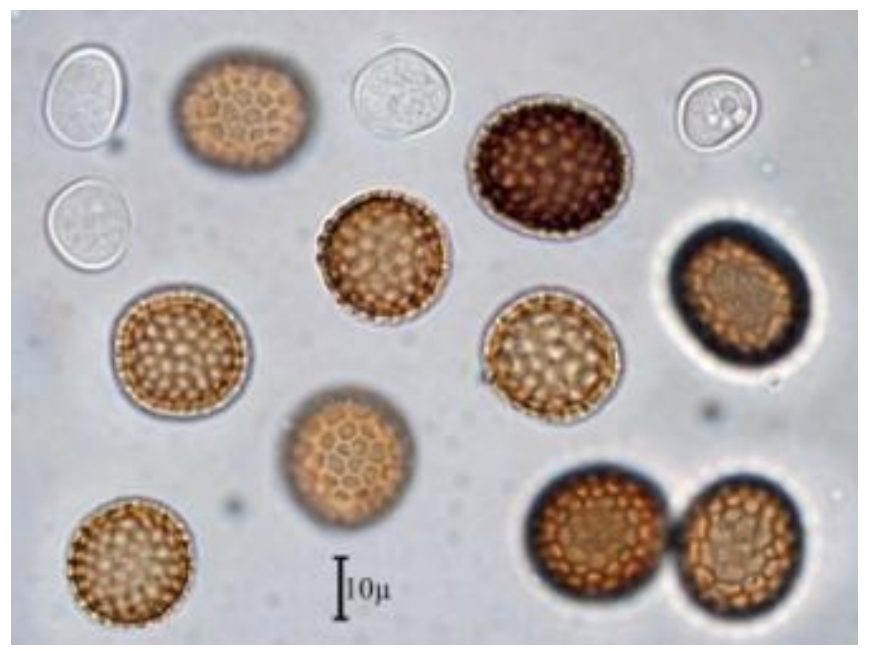

Fig.42 - Tilletia lolioli on Loliolum subulatum (10548) 


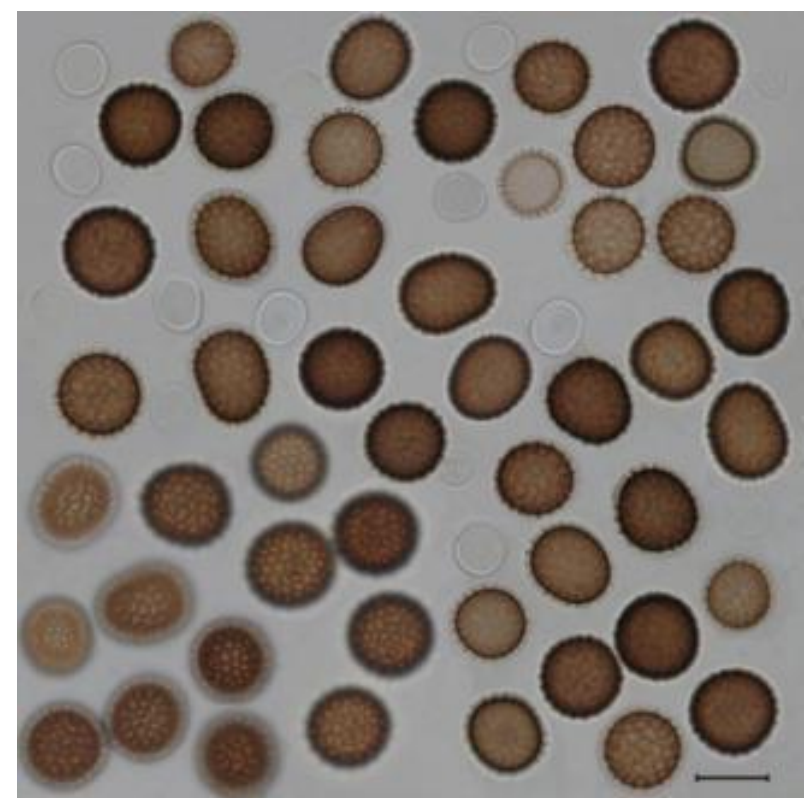

Fig.43 - Tilletia rostrariae on Rostraria cristata (7260), Bar=20 $\mu \mathrm{m}$

\section{Tranzscheliella Lavrov}

\section{Key to the Tranzscheliella species}

1. Spores in LM with 2, large, bipolar appendages, in SEM operculate T. williamsii

- Spores not so

2. Sori in culms surrounding the upper internodes; spores $4-6(-7) \mu \mathrm{m}$ long; wall c. $0.5 \mu \mathrm{m}$ thick, smooth

T. hypodytes

- Sori destroying the entire inflorescence; spores 5-8 $\mu \mathrm{m}$ long; wall 0.5-1.5 $\mu \mathrm{m}$ thick, from apparently smooth to finely, densely verrucose T. iranica

55. Tranzscheliella hypodytes (Schltdl.) Vánky \& McKenzie, Smut Fungi of New Zealand: 156, 2002, s. lat.

Caeoma hypodytes Schltdl. 1824. - Ustilago hypodytes (Schltdl.) Fries 1832. - Erysibe hypodytes (Schltdl.) Wallr. 1833. - Uredo hypodytes (Schltdl.) Desm. 1840. - Cintractia hypodytes (Schltdl.) Maire 1906.

Ustilago hypodytes var. lolii Thüm. 1874.

Ustilago sporoboli Ellis \& Everh. 1897 (later homonym; not U. sporoboli Tracy \& Earle 1896). Ustilago funalis Ellis \& Everh. 1897 (nom. nov.).

Ustilago nummularia Speg. 1902.

Ustilago stipicola Speg. 1902.

Ustilago dactylidis Maire 1917.

Ustilago athenae Maire 1917.

Ustilago agrestis Syd. 1924. - Ustilago spegazzinii Hirschh. var. agrestis (Syd.) G.W. Fisch. \& Hirschh. 1945.

Ustilago bromi-erecti Cif. 1931.

Ustilago sumnevicziana Lavrov 1936.

Ustilago spegazzinii Hirschh. 1939a.

Ustilago custanaica Lavrov 1951.

Ustilago hypodytes f. sporoboli Zambett. 1980 '1979' (nom. inval.). 
Sori in culms as a blackish brown, semi-agglutinated to powdery spore mass surrounding the upper internodes (extending from the basal part of the internode sometimes to the next node) and occasionally in the axis of an abortive inflorescence. Sori initially protected by the leaf sheath, finally more or less naked, peridium absent. Upper internodes and leaves of host usually stunted. Infection systemic, inflorescence usually abortive. Spores globose, subglobose to ovoid, occasionally elongate, irregular or slightly flattened, 3.5-5.5 $\times 4-6(-7) \mu \mathrm{m}$, medium to dark olivebrown; wall c. $0.5 \mu \mathrm{m}$ thick, smooth, usually with a small, inconspicuous, hyaline, smooth or finely punctate-verruculose cap at the poles, in SEM densely, minutely, uniformly verruculose on the entire surface. Spore germination results in slender, septate (three- or 4-celled, four-nucleate) basidia developing lateral, ramifying, septate, uninucleate branches producing, on nutrient media, clumps of aerial sporidia. Rarely ovoid basidiospores are produced on sterigmata, at the septa of the basidia (Fig. 44).

On Poaceae: Elymus hispidus (Opiz) Melderis (Agropyron intermedium (Host) P. Beauv.), Ardebil Prov., between Sarab and Ardebil city.

On Elymus hispidus subsp. barbulatus (Schur) Melderis (Agropyron trichophorum K. Richt.; A. intermedium (Host) P. Beauv.), Golestan Prov., Golestan National Park, Almeh, IRAN 8128 F.

On Elymus repens (L.) Gould (Agropyron repens (L.) P. Beauv.), Tehran Prov., Karaj city, IRAN 7299 F; West Azarbaijan Prov., Oshnavieh city, IRAN 8069 F.

On Elymus sp., Tehran Prov., Central Alborz, Azadbar village, IRAN 10500 F.

On Stipa parviflora Desf., Hormozgan Prov., Anveprody Mt., at Bastak city; Kerman Prov., Mahan city, IRAN 8292 F.

56. Tranzscheliella iranica (Syd.) Vánky, Mycotaxon 106: 137; 2008.

Ustilago iranica Syd. 1939.

Sori destroying the entire inflorescence, partly enclosed by the uppermost leaf sheaths, up to $7 \mathrm{~cm}$ long, with black or blackish brown, powdery spore mass, peridium absent. Spores subglobose, broadly ellipsoidal or rounded subpolyhedrally slightly irregular, 4-7 $\times 5-8 \mu \mathrm{m}$, yellowish- to olivebrown; wall $0.5-1.5 \mu \mathrm{m}$ thick, from apparently smooth to finely, densely verrucose, spore profile smooth to finely wavy, no polar caps, in SEM with rounded, low warts fusing into irregular groups or short, irregular rows (Fig. 45).

On Poaceae: Stipa tenerrima Bornm. \& Gauba, Alborz Mountains, Nemar Mt., 3.VI.1937, leg. D.E. Gauba 1233.

On Stipa sp., Yazd Prov., Dehe bala village, IRAN 14697 F.

Remarks - Tranzscheliella iranica is still "endemic" for Iran, known from only two localities.

57. Tranzscheliella williamsii (Griffiths) Dingley \& Versluys, New Zealand J. Bot. 15: 477, 1977.

Sorosporium williamsii Griffiths 1902. - Ustilago williamsii (Griffiths) Lavrov 1936. - Ustilago williamsii (Griffiths) G.W. Fisch. \& Hirschh. 1945 (comb. superfl.).

Ustilago appendiculata Speg. 1909. - Tranzscheliella appendiculata (Speg.) Lavrov 1936.

Tranzscheliella otophora Lavrov 1936. - Ustilago otophora (Lavrov) Gutner 1941.

Sori surrounding upper internodes and aborted inflorescence axes, initially protected by leaf sheaths, later naked, blackish brown, powdery, lacking a peridium. Infection systemic, infected plants usually not flowering. Spores globose to subglobose, 6-8 $\times 7-10 \mu \mathrm{m}$, olive-brown, exospore often deeply cracked, bearing two, hyaline, winged or cup-like, 1-2.5 $\mu \mathrm{m}$ thick, bipolar appendages, in SEM typically operculate. Spore germination results in 4-celled, often branched basidium producing elongate basidiospores (Fig. 46).

On Poaceae: Stipa hohenackeriana Trin. \& Rupr., Esfahan Prov., 40-45 km S of Esfahan city, Kolah-Ghazi National Park, IRAN 8317 F.

On Stipa pulcherrima K. Koch, Golestan Prov., Gorgan city, Golestan National Park, IRAN $8298 \mathrm{~F}$. 


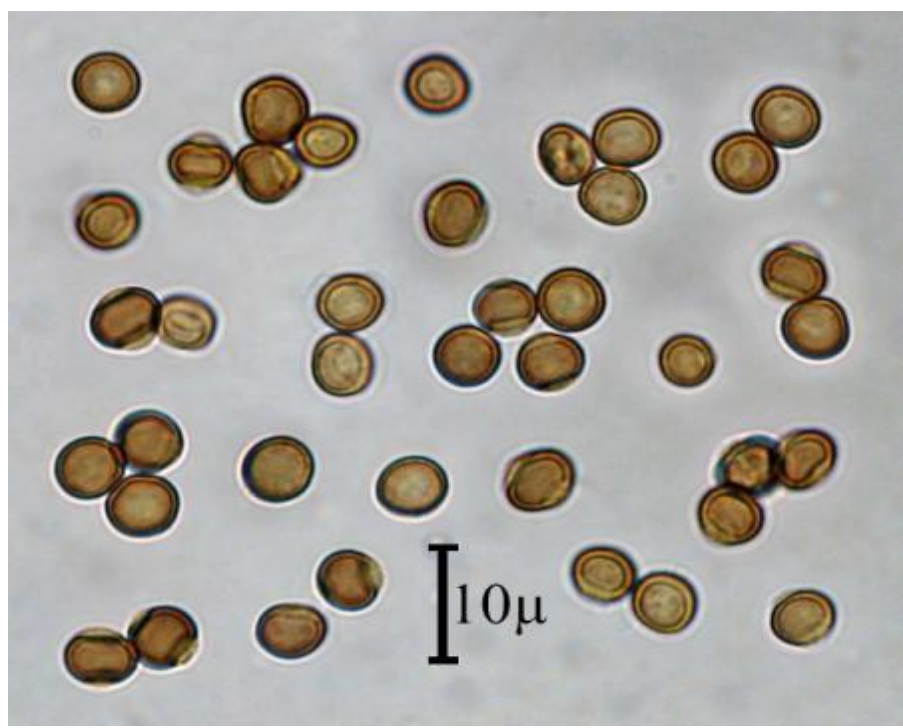

Fig.44 - Tranzscheliella hypodytes on Elymus sp. (10500)

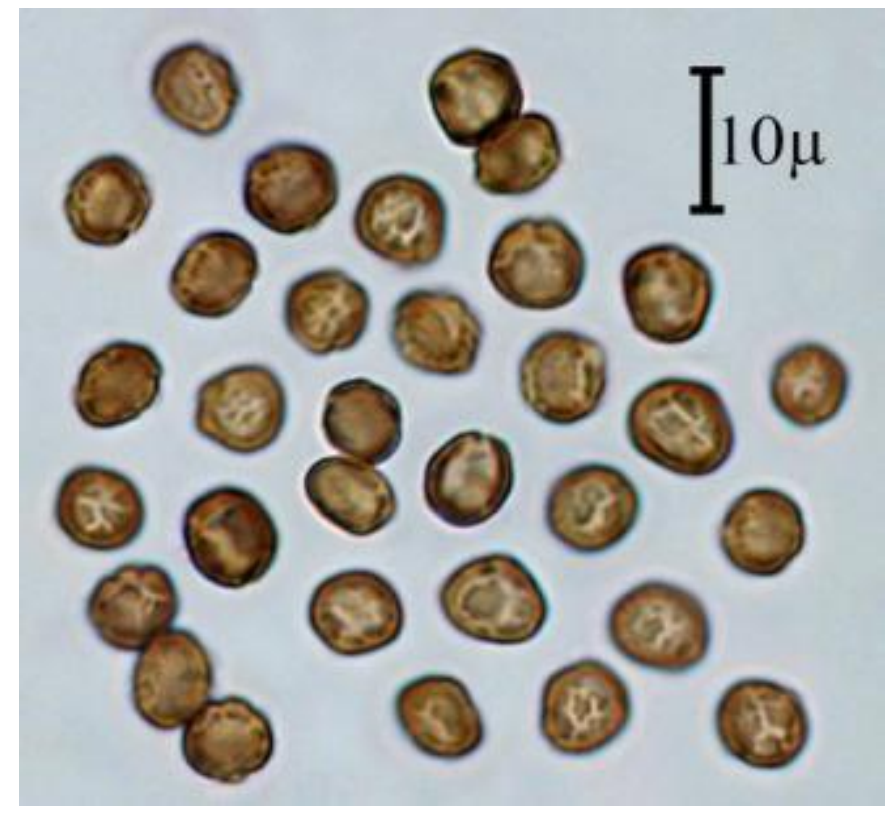

Fig.45 - Tranzscheliella iranica on Stipa sp. (14697)

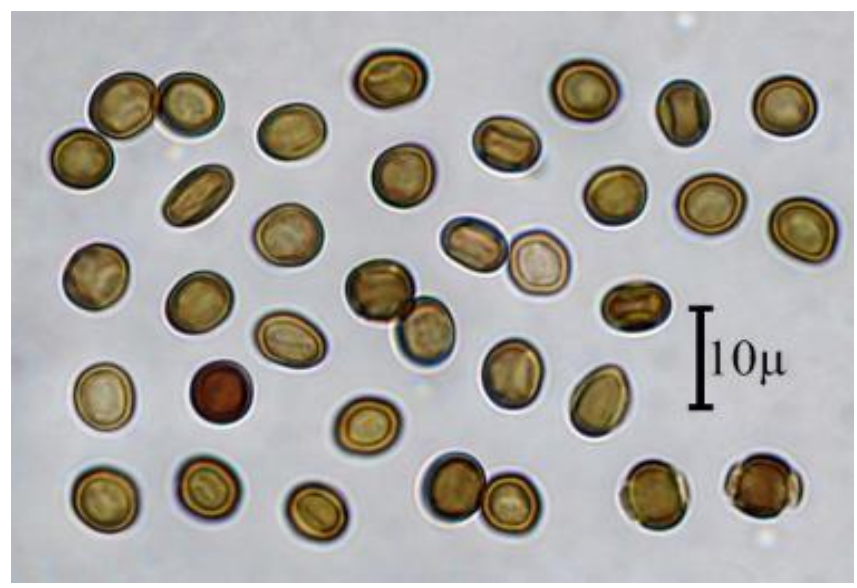

Fig.46 - Tranzscheliella williamsii on Stipa hohenackeriana (8317) 
Key to the Urocystis species based on host plant taxonomy

1. On Alliaceae (Allium) Ur. magica

- Not on Alliaceae .2

2. On Amaryllidaceae (Ixiolirion) Ur. ixiolirii

- Not on Amaryllidaceae 3

3. On Colchicaceae (Colchicum) Ur. colchici

- Not on Colchicaceae 4

4. On Cyperaceae (Carex) Ur. fischeri

- Not on Cyperaceae. . .5

5. On Hyacinthaceae ..6

- Not on Hyacinthaceae 7

6. On Muscari Ur. muscaridis

- On Ornithogalum Ur. ornithogali

7. On Iridaceae (Gladiolus). Ur. gladiolicola

- Not on Iridaceae ..8

8. On Orobanchaceae (Orobanche) Ur. orobanches

- Not on Orobanchaceae ..9

9. On Poaceae. .10

- Not on Poaceae 16

10. On Agropyron

- Not on Agropyron .

11. On Arrhenatherum ri

- Not on Arrhenatherum ...Ur. behboudii

12. On Critesion Ur. tianschanica

- Not on Critesion .13

13. On Lolium Ur. bolivarii

- Not on Lolium .14

14. On Phalaris Ur. phalaridis

- Not on Phalaris .15

15. On Stipa Ur. corsica

- On Triticum. Ur. tritici

16. On Primulaceae (Primula)

- Not on Primulaceae Ur. primulae

17. On Ranunculaceae. .17

- Not on Ranunculaceae.

18. On Ceratocephalus . 18

- Not on Ceratocephalus... 21

19. On Ranunculus

- On Thalictrum. 20

20. Spore balls surrounded by a continuous or discontinuous layer of sterile cells ...Ur. ficariae

- $\quad$ Sterile cells few or lacking Ur. ranunculi

21. On Scrophulariaceae (Pedicularis) Ur. pedicularis

- On Violaceae (Viola) 22

22. Sori in flowers and ovaries; spore balls composed of $1-8(-9)$ spores Ur. kmetiana

- Sori on petioles and leaves; spore balls composed of (1-)3-20 or more spores .... Ur. violae

58. Urocystis agropyri (Preuss) A.A. Fisch. Waldh., Bull. Soc. Imp. Naturalistes Moscou 40: 258, 1867. 
Uredo agropyri Preuss, in Sturm 1848. - Polycystis agropyri (Preuss) J. Schröt., in Cohn 1877. Tuburcinia agropyri (Preuss) Liro 1922.

Urocystis preussii J.G. Kühn, in Rabenh. 1874.

Tuburcinia elymi Cif. 1931. - Urocystis elymi (Cif.) Schwarzman 1960.

Tuburcinia agropyri-juncei Vienn.-Bourg. 1954 '1953' (nom. inval.). - Urocystis agropyri-juncei (Vienn.-Bourg.) H. Zogg 1986 '1985' (comb. illegit.).

Sori in leaves, sheaths and culms, often also in the rachis of the aborted inflorescence, as short to long streaks parallel to the veins, initially lead-coloured and covered by the epidermis which soon ruptures to expose the dark brown, dusty spore mass. Spore balls globose to elongate, 16-32 $\mu \mathrm{m}$ long, composed of 1-3(-4) spores and a completely or almost completely investing layer of sterile cells. Spores globose, subglobose or ovoid, sometimes with flattened sides, 9.5-15 × 12-17.5 $\mu \mathrm{m}$, reddish brown, smooth. Sterile cells hyaline to yellowish, 4-12 $\mu \mathrm{m}$ long; wall thin, c. $1 \mu \mathrm{m}$, collapsing with age giving a ridged appearance to the spore ball surface, in SEM very finely punctate-verruculose. Spore germination results in aseptate basidia, usually with four, apical, cylindrical basidiospores that fuse in pairs to form dikaryotic hyphae.

On Poaceae: Agropyron intermedium (Host) P. Beauv., Zanjan Prov.

59. Urocystis behboudii (Esfand.) Vánky, Symb. Bot. Upsal. 24(2): 154, 1985a.

Tuburcinia behboudii Esfand., in Esfand. \& Petrak 1950.

Sori in leaves forming striae between the veins, initially covered by the epidermis which ruptures longitudinally exposing the black, semi-agglutinated to powdery mass of spore balls. Spore balls subglobose, ovoid, ellipsoidal to slightly irregular, 20-32(-36) $\times 21-40(-48) \mu \mathrm{m}$, composed of $1-$ 4(-5) spores and a completely investing layer of sterile cells. Spores globose, ellipsoidal, elongate or irregular, 13-17 × 14.5-21.5 $\mu \mathrm{m}$, reddish brown. Sterile cells subglobose, ellipsoidal or irregular, 6$13 \mu \mathrm{m}$ long, yellowish brown; wall uneven, $0.5-1 \mu \mathrm{m}$ thick, smooth.

On Poaceae: Arrhenatherum kotschyi Boiss., Kermanshah Prov., Ghalachjeh Mt., Ghalachjeh pass, GZU (holotype), HUV 14807, IRAN 4398 F.

60. Urocystis bolivarii Bubák \& Gonz. Frag., in Bubák, Bol. Soc. Españ. Hist. Nat. 22: 205. 1922. (as 'bolivari').

Tuburcinia bolivarii (Bubák \& Gonz. Frag.) Gonz. Frag., in Cif. 1933 (as 'bolivari'). - Tuburcinia bolivarii (Bubák \& Gonz. Frag.) Uljan., in Lavrov 1938 (comb. superfl.). - Tuburcinia occulta [var.] bolivarii (Bubák \& Gonz. Frag.) Cif. 1963 (as 'bolivari'; comb. illegit.).

Tuburcinia lolii Kawecka-Starmachowa 1939.

Sori in leaves, sheaths, culms and inflorescence as long streaks between the veins, initially leadcoloured, covered by the epidermis which soon ruptures exposing the blackish brown, powdery mass of spore balls. Spore balls globose, ovoid to irregular, 16-44 $\mu \mathrm{m}$ in diam., composed of 1-6 spores almost completely invested by sterile cells. Spores globose, ovoid, sometimes irregular, 10.5-16 $\times$ 13-18.5 $\mu \mathrm{m}$, pale brown, smooth. Sterile cells globose to irregular, 5-12 $\mu \mathrm{m}$, yellowish brown (Fig. 47).

On Poaceae: Lolium loliaceum (Bory \& Chaub.) Hand.-Mazz., Golestan Prov., Gorgan city, Golestan National Park, Abshar area, IRAN 8324 F.

On Lolium rigidum Gaudin, Ardebil Prov., Moghan city, between Ultan village \& Ghara-aghaj village, IRAN 8592 F; Golestan Prov., Gorgan city, $150 \mathrm{~km}$ W. Bojnourd city, $34 \mathrm{~km} \mathrm{SW}$ of Maraveh tappeh village, IRAN 7269 F; Ardebil Prov., Moghan city, between Germi village \& Parsabad village, IRAN $8591 \mathrm{~F}$.

On Lolium sp., Sistan va-Baluchestan Prov., Saravan city, Suran village, IRAN 10339 F.

61. Urocystis ceratocephali Zambett. ex Vánky, Mycotaxon 33: 370, 1988 b.

Tuburcinia ceratocephali Zambett. 1971 (nom. inval.).

Sori on the stems forming fusiform swellings up to $1 \mathrm{~cm}$ long, covered by a greyish silvery membrane which ruptures longitudinally exposing the black, granular-dusty mass of spore balls. 
Spore balls subglobose, elongate or rounded irregular, 16-35 $\times 20-45 \mu \mathrm{m}$, composed of 1-7 spores surrounded by an incomplete layer of, sometimes only a few, sterile cells. Spores subglobose to ellipsoidal, often flattened on contact sides, 11-15 × 14-20 $\mu \mathrm{m}$, dark reddish brown, smooth. Sterile cells subglobose, elongate to pyriform and irregularly flattened, 5-12(-14) $\mu \mathrm{m}$ long, pale yellowish brown, smooth (Fig. 48).

On Ranunculaceae: Ceratocephalus falcatus (L.) Pers., Esfahan Prov., on the way between Shahreza and Semirom cities, IRAN 7238 F.

62. Urocystis colchici (Schltdl.) Rabenh., Fgi. eur. no. 396, 1861.

Caeoma colchici Schltdl. 1826. - Uredo colchici (Schltdl.) Endl. 1830. - Sporisorium colchici

(Schltdl.) Libert, 1832. - Polycystis colchici (Schltdl.) Lév. 1847. - Polycystis colchici (Schltdl.)

F. Strauss, in Sturm 1853 (comb. superfl.). - Tuburcinia colchici (Schltdl.) Liro 1922.

Erysibe arillata Wallr. var. colchici Wallr. 1833.

Urocystis colchici-lutei Zundel 1944.

Sori in leaves and petioles as slightly elevated, pustular, elongate areas of various size and shape, often confluent, visible on both sides of the leaf, initially lead-coloured and covered by the epidermis which ruptures exposing the powdery, black mass of spore balls. Infection systemic. Spore balls globose, subglobose, ovoid to irregular, (16-)20-40 $\mu \mathrm{m}$ in diam., composed of 1-3(-4) spores and a more or less complete investing layer of sterile cells. Spores globose, subglobose, ovoid, irregularly oblong or subpolyhedral, 9-14.5(-15) $\times(11-) 12-20(-23) \mu \mathrm{m}$, medium reddish brown, smooth. Sterile cells subglobose, ovoid to irregular, 5-10 × 6-15 $\mu \mathrm{m}$, pale yellow, with smooth, 1-2 $\mu \mathrm{m}$ thick wall, collapsed in old specimens (Fig. 49).

On Colchicaceae (Liliaceae s. lat.): Colchicum persicum Baker, Golestan Prov., Jahannama protected region, IRAN $8329 \mathrm{~F}$.

On Colchicum procurrens Baker, Tehran Prov., Karaj city.

On Colchicum sp., Khorasan Prov., 10 km E of Bojnourd city, Baba-Aman area. IRAN 7268 F; Tehran Prov., Lavasan city, at Latian dam, IRAN 8215 F; Kordestan Prov., Dalahu Mt., Rijab city, IRAN 8327 F; Mazandaran Prov., IRAN 7128 F; Tehran Prov., Tehran city, Central Albroz, Darabad area, IRAN $8032 \mathrm{~F}$.

63. Urocystis corsica (Mayor \& Terrier) Vánky, Publ. Herb. Univ. Uppsala 9: 12, 1982. Sorosporium corsicum Mayor \& Terrier 1959. - Tuburcinia corsica (Mayor \& Terrier) Guyot \& Massenot, in Guyot, Malençon \& Massenot 1969.

Sori in congested uppermost leaves and in more or less aborted inflorescence as long, dark leadcoloured striae between the leaf veins, often covering the entire leaf surface, initially protected by a delicate, silvery membrane which ruptures exposing the black, powdery mass of spore balls. Spore balls globose, ellipsoidal to elongate ovoid, 30-50 × 35-65(-75) $\mu \mathrm{m}$, dark chestnut brown, opaque, composed of (3-)6-15(-20?) spores surrounded by a continuous layer of sterile cells. Spores subglobose, ellipsoidal, elongate or slightly irregular by mutual pressure, 10-14 × 12-16(-20) $\mu \mathrm{m}$, dark reddish brown, smooth. Sterile cells variable in shape and size, (5-)7-20 $\mu \mathrm{m}$ long, thick-walled (1.5-3 $\mu \mathrm{m}$ ), thickest at their basal and lateral parts (Fig. 50).

On Poaceae: Stipa capensis Thunb. (S. tortilis Desf.), Gilan Prov., N. shore of White River dam, IRAN 7239 F; Bushehr Prov., Dashtestan County, Borazjan city, IRAN 8296 F.

64. Urocystis ficariae (Liro) Moesz, Budapest és környékének gombái: 137, 1942.

Caeoma ficariae "Schltdl." sensu Unger 1833 (non sensu orig., q.e. Uromyces ficariae Lév.). Tuburcinia ficariae "(Unger)" Liro 1922. - Urocystis ficariae "(Unger)" Zundel 1953. Urocystis ficariae "(Unger)" D.M. Hend. 1955 (comb. superfl.).

Sori in leaves and petioles as pustular swellings, initially covered by the epidermis which soon ruptures revealing the black, powdery mass of spore balls. Spore balls globose to ovoid or irregular, 20-32 × 24-40(-44) $\mu \mathrm{m}$, composed of 1-3(-4) central spores and a discontinuous to continuous layer of peripheral sterile cells. Spores globose to ovoid, dark reddish brown, 11-17.5 × 13-20 $\mu$ m; 
wall even, c. $0.8 \mu \mathrm{m}$ thick, smooth. Sterile cells globose, hemiglobose to elongate or irregular, pale yellowish brown, 5-11 × 5.5-15 $\mu \mathrm{m}$, smooth (Fig. 51).

On Ranunculaceae: Ranunculus ficaria L. (Ficaria ranunculoides Roth), Tehran Prov., Haraz road, Emamzadeh Hashem shrine, IRAN 7236 F; Golestan Prov., 37 km SW of Gorgan city; Kordkuy city, Deraznu forest, IRAN 7237 F; Esfahan Prov., 140 km NW of Esfahan city, Khonsar city; Golestan Mt., IRAN 7266 F; Mazandaran Prov., Chalus road, Siahbisheh village, IRAN 10478 F; Chaharmahal va-Bakhtiari Prov., Chelgerd city, Kuhrang Dam, IRAN 8209 F.

65. Urocystis fischeri Körn. ex G. Winter, Rabenh. Krypt.-Fl., 2 Aufl. 1(1): 120, 1881.

Urocystis fischeri Körn. 1877b (nom. nud.). - Tuburcinia fischeri (Körn. ex G. Winter) Liro 1922.

Urocystis caricis Ule 1884.

Tuburcinia rigida Liro 1939. - Urocystis rigida (Liro) Zundel 1953.

Sori in leaves, rarely in culms as short to long, inflated streaks between the veins, sometimes confluent, initially lead-coloured, covered by the epidermis which ruptures longitudinally to expose the powdery, dark brown mass of spore balls. Spore balls globose, ovoid to irregular, 20-40 $\mu \mathrm{m}$, composed of 1-3(-4) spores completely invested by a layer of sterile cells. Spores globose to elongate or slightly irregular, polyhedral, 11-16 × 14.5-19 $\mu \mathrm{m}$, light to dark reddish brown, smooth. Sterile cells globose, hemiglobose, elongate to irregular, 5-15 $\mu \mathrm{m}$ in diam., pale yellowish brown, smooth (Fig. 52).

On Cyperaceae: Carex sp., Tehran Prov., Karaj-Chalus road, Jei \& Charan bifurcation, bogs at the Gholgholak spring, IRAN 10908 F.

66. Urocystis gladiolicola Ainsw., Trans. Brit. Mycol. Soc. 32: 257, 1949.

Tuburcinia gladiolicola (Ainsw.) Cif. 1963 (non Uredo gladioli Req.).

Sori in leaves, scapes and scales of the bulb as greyish brown, blister-like, $1 \mathrm{~mm}$ to several $\mathrm{cm}$ long striae between the veins, initially covered by the epidermis which ruptures exposing the dark brown, powdery mass of spore balls. Infection systemic; mycelium hybernating in the corms. Spore balls globose, ovoid, occasionally elongate, $16-23 \times 18-30(-35) \mu \mathrm{m}$, each composed of 1-2(-3) spores, completely invested by an irregular layer of sterile cells. Spores globose, ovoid to slightly angular, $12-15 \times 12-18 \mu \mathrm{m}$, reddish brown. Sterile cells pale yellowish brown, 6-12 $\mu \mathrm{m}$ in diam., collapsing with age (Fig. 53).

On Iridaceae: Gladiolus segetum Ker Gawl., Kohgiluyeh va-Buyerahmad Prov., Dehdasht city, Tol-Chogha area, IRAN $8008 \mathrm{~F}$.

67. Urocystis ixiolirii Zaprom., Materialy po mikoflore Srednei Azii 2: 23, 1928.

Tuburcinia ixiolirii (Zaprom.) Lavrov 1937.

Sori in leaves as pustules, 1-2 $\times 6-10 \mathrm{~mm}$, initially covered by a greyish membrane of host tissue which ruptures at maturity exposing the semi-powdery, dark brown mass of spore balls. Spore balls globose, ellipsoidal to slightly irregular, 22-35 × 25-50 $\mu \mathrm{m}$, composed of 1-2(-3) spores, completely surrounded by sterile cells. Spores globose, ovoid, ellipsoidal to slightly irregular with flattened sides, 12-16 × 15-20 $\mu \mathrm{m}$, reddish brown; wall evenly thick, c. $0.5 \mu \mathrm{m}$, smooth. Sterile cells irregular, 5-12 $\mu \mathrm{m}$ long, yellow, collapsed in old specimens; wall unevenly thick, 1-2 $\mu \mathrm{m}$, smooth (Fig. 54).

On Amaryllidaceae: Ixiolirion pallasii Fisch. \& C.A. Mey. ex Ledeb. (I. tataricum Roem. \& Schult.; I. montanum (Lab.) Herb.), Kerman Prov., Khan-Sorkh Pass, IRAN 4396 F; Sistan vaBaluchestan Prov., Chah-Bahar port, IRAN 4397 F; Sistan va-Baluchestan, Khash city, Tamandan village, IRAN $10424 \mathrm{~F}$.

On Ixiolirion sp., Kerman Prov., Kerman city.

68. Urocystis kmetiana Magnus, Verh. Bot. Vereins Prov. Brandenburg 31: 19, 1890.

Tuburcinia kmetiana (Magnus) Liro 1922. 
Sori in flowers and ovaries which are destroyed although the calyces usually remain intact, occasionally also producing distortion in the stems, 3-5 $\mathrm{mm}$ in diam. Spore mass powdery, black. Spore balls globose to ovoid, 28-36 × 28-44(-52) $\mu \mathrm{m}$, composed of 1-8(-9) central spores and a complete investing layer of peripheral sterile cells. Spores subglobose, ovoid to slightly polyhedral, (9.5-)10.5-13 × 11-20 $\mu \mathrm{m}$, reddish brown; wall smooth, c. $0.8 \mu \mathrm{m}$ thick. Sterile cells globose, ovoid to irregular, 5.5-14.5 $\mu \mathrm{m}$ in diam., yellowish brown, smooth, collapsing with age (Fig. 55).

On Violaceae: Viola occulta Lehm., Tehran Prov., Karaj-Chalus road, 20 km NE of Karaj city, near "Amir-Kabir" dam, IRAN 7235 F; Quazin Prov., 50 km NW of Qazvin city, IRAN 7234 F.

On Viola tricolor L., Kermanshah Prov., Tange Chovar, IRAN 6561 F.

69. Urocystis magica Pass., in Thüm., Mycoth. univ. no. 223, 1875, s. lat.

Tuburcinia magica (Pass.) Liro 1922.

Urocystis cepulae Frost, in Farlow 1877. - Tuburcinia cepulae (Frost) Liro 1922.

Urocystis colchici (Schltdl.) Rabenh. f. allii-subhirsuti Beltrani, in Thümen 1878.

Urocystis allii Schellenb. 1911. - Tuburcinia allii (Schellenb.) Liro 1922.

Tuburcinia oblonga Massenot 1953. - Urocystis oblonga (Massenot) H. Zogg 1986.

Sori in leaves and bulbs as pustules, often confluent, $1 \mathrm{~mm}$ to several $\mathrm{cm}$ long, initially covered by the epidermis which ruptures to expose the dusty, blackish brown mass of spore balls. Spore balls globose to ovoid, composed of 1-2 central spores and a discontinuous to continuous layer of peripheral sterile cells. Spores globose, subglobose, ovoid to slightly irregular, 10.5-13.5 × 13-16(19) $\mu \mathrm{m}$, medium to dark reddish brown. Sterile cells globose, ovoid to irregular, 5-10 $\mu \mathrm{m}$ in diam., pale yellowish brown. In SEM spores and sterile cells sparsely to moderately densely, very finely verruculose. Spore germination results in a hemispherical or shortly cylindrical, aseptate basidium from which 4-8, septate, ramified hyphae arise (Fig. 56).

On Alliaceae (Liliaceae s. lat.): Allium akaka Gmel. ex Roem. \& Schult, reported by Khabiri (1958), without mentioning locality.

On Allium cepa L., East Azarbaijan Prov., Tabriz, IRAN 4391 F.

70. Urocystis muscaridis (Niessl) Moesz, A Kárpát-medence üszöggombái: 199, 1950.

Polycystis colchici (Schltdl.) Lév. var. muscaridis Niessl 1861. - Tuburcinia muscaridis (Niessl)

Liro 1922. - Urocystis muscaridis (Niessl) Zundel 1953 (comb. superfl.).

Urocystis colchici (Schltdl.) G. Winter f. muscari Bizz. 1885.

Urocystis colchici "Strauss" f. muscari-comosi Thüm. 1874 (nom. nud).

Sori in leaves as ellipsoidal, 2-10 mm long pustules, covered by the lead-coloured epidermis, until it ruptures longitudinally and the black, powdery mass of spore balls becomes scattered. Spore balls globose, ovoid to irregular, 20-40 × 20-48 $\mu \mathrm{m}$, composed of 1-5(-9) spores surrounded by a continuous layer of sterile cells. Spores globose, ovoid to irregular, often slightly polyhedral, 10.5$16 \times 14-22.5(-24) \mu \mathrm{m}$, dark reddish brown. Sterile cells variable in shape and size, globose, ovoid to irregular, 4-12 $\times$ 6-20 $\mu \mathrm{m}$, yellowish brown, smooth (Fig. 57).

On Hyacinthaceae (Liliaceae s. lat.): Muscari sp., Khuzestan Prov., Baghmalek city, IRAN $11799 \mathrm{~F}$.

71. Urocystis ornithogali Körn. ex A.A. Fisch. Waldh., Ann. Sci. Nat. Bot. 4: 240, 1877a1876'; Aperçu Syst. Ustil.: 41, 1877b.

Tuburcinia ornithogali (Körn.) Liro 1922. - Tuburcinia ornithogali (Körn.) S. Ito 1935 (comb. superfl.).

Urocystis hypogaea Körn., in Fuckel 1876 (nom. nud.). - Tuburcinia hypogaea (Körn.) S. Ito, in Ito \& Homma 1938 (comb. illegit.).

Sori in leaves and stalks as ovoid, small to large pustules and swellings, initially lead-coloured and covered by the epidermis which ruptures and the powdery, black mass of spore balls becomes scattered. Spore balls globose, subglobose to ovoid, 20-32 × 23-44 $\mu \mathrm{m}$, composed of 1-3(-4) central spores and a completely investing layer of sterile cells. Spores globose, subglobose to ovoid, 
sometimes with a flattened side, 11-17 × 14.5-20 $\mu \mathrm{m}$, dark reddish brown. Sterile cells globose, ovoid to irregular, 5-13 $\mu \mathrm{m}$ in diam., with thick (1-2 $\mu \mathrm{m})$, smooth, yellow or yellowish brown wall, collapsing with age (Fig. 58).

On Hyacinthaceae (Liliaceae s. lat.): Ornithogalum kurdicum Bornm., Lorestan Prov., Khorramabad city, Hashtad-Pahlou Mt., IRAN 11658 F.

72. Urocystis orobanches (Mérat) A.A. Fisch. Waldh., Aperçu Syst. Ustil.: 42, 1877.

Rhizoctonia orobanches Mérat 1821. - Tuburcinia orobanches (Mérat) Fr. 1832. - Ustilago orobanches (Mérat) Lév. 1846. - Polycystis orobanches (Mérat) Lév. 1847. - Thecaphora orobanches (Mérat) Lév. in d'Orbigny 1849. - Schinzia orobanches (Mérat) Cocc., in Cif. 1938.

Sori in roots and underground stem, swollen, filled with a blackish brown, powdery mass of spore balls. Spore balls globose, ellipsoidal to rounded irregular, 20-30 × 24-40 $\mu \mathrm{m}$, composed of 1-2(-3) spores completely surrounded by one, or in places two layers of sterile cells. Spores globose to ovoid, 9-14 × 11-18 $\mu \mathrm{m}$, dark reddish brown, smooth. Sterile cells subglobose, ovoid to irregularly elongate, $4-8 \times 5-13 \mu \mathrm{m}$, pale brown, smooth.

On Orobanchaceae: Orobanche ramosa L.

Reported by Magnus (1893) on Orobanche (Phelipaea) sp., Bushehr Prov., Bushehr city.

73. Urocystis pedicularis (Golovin) Vánky, Mycotaxon 99: 48, 2007.

Sorosporium pedicularis Golovin, in Gutner 1941 (nom. inval.). - Sorosporium pedicularis Golovin 1950.

Sori in all capsules of an inflorescence, swollen, deformed, filled with blackish brown, granularpowdery mass of spore balls. Spore balls subglobose, ovoid, ellipsoidal, elongate to irregular, 25-75 $\times 35-90 \mu \mathrm{m}$, reddish brown, composed of a few to tens of spores that separate under pressure. Spores variable in shape and size, subglobose, ovoid, ellipsoidal, elongate, irregular, subcuneiform, 8-14.5 × 10.5-22 $\mu \mathrm{m}$, reddish brown; wall even, 0.5-1 $\mu \mathrm{m}$ thick, apparently smooth, in SEM very finely, low verruculose. Sterile cells lacking (Fig. 59).

On Scrophulariaceae: Pedicularis sibthorpii Boiss., Mazandaran Prov., Haraz road, Nava village, IRAN $8312 \mathrm{~F}$.

Remarks - Typical for Urocystis pedicularis is the lack of sterile cells around the spores.

74. Urocystis phalaridis Vánky, in Vánky \& Abbasi, Rostaniha 12(2):189, 2012b.

Sori on leaves as long striae between the veins, at first lead coloured, covered by the epidermis which ruptures longitudinally disclosing the dark reddish brown, powdery mass of spore balls. Spore balls subglobose, irregular to elongated, 20-40 × 25-50 $\mu \mathrm{m}$, composed of 1-7(-9) spores and a completely investing layer of sterile cells. Spores subglobose, ovoid, ellipsoidal, elongated or subpolyhedrally irregular, with one or several flattened sides, 9-15 $\times 11-19 \mu \mathrm{m}$, reddish brown. Sterile cells subglobose, ellipsoidal, elongated or irregular, 6.5-14 $\mu \mathrm{m}$ long, yellow; wall uneven, $0.5-2.5 \mu \mathrm{m}$ thick, thinner on the free surface, thick on the contact sides, in SEM finely verruculose (Fig. 60).

On Poaceae: Phalaris sp. Golestan Prov., Gorgan, 1.I.1993, leg. Sh. Daemi, holotypus HUV 21977, isotypus IRAN $10352 \mathrm{~F}$. Known only from the type locality.

75. Urocystis primulae (Rostr.) Vánky, Symb. Bot. Upsal. 24(2): 176, 1985a.

Sorosporium primulae Rostr. in A.A. Fisch. Waldh. 1879a. - Tuburcinia primulae (Rostr.) Liro 1922. - Ginanniella primulae (Rostr.) Cif. 1938.

Sori in ovaries; when the seed pods open at their tops, the black, powdery mass of spore balls becomes scattered. Spore balls globose, ovoid to elongate, 32-60 × 40-88 $\mu \mathrm{m}$, composed of (1-)315 (or more) spores completely invested by a layer, sometimes in places two layers, of sterile cells which often collapse upon drying and resemble an enveloping membrane around the spores. Spores globose, subglobose, ovoid to elongate or irregular, somewhat angular, 11-16(-20) $\times 12-21 \mu \mathrm{m}$, medium to dark reddish brown; wall c. $0.8 \mu \mathrm{m}$ thick, smooth. Sterile cells variable in shape, size, 
colour and wall thickness; globose, subglobose, ovoid, elongate, pyriform to irregular, 5.5-14.5 × 8$17.5 \mu \mathrm{m}$, yellow to pale reddish brown; wall 1-3 $\mu \mathrm{m}$ thick, smooth. Spore formation preceded by a farinose, white anamorph in the flowers (Paepalopsis irmischiae J.G. Kühn).

On Primulaceae: Primula veris subsp. macrocalyx (Bunge) Lüdi (Primula macrocalyx Bunge), Tehran Prov., Chalous road, 55 km NE of Karaj city.

76. Urocystis ranunculi (Lib.) Moesz, A Kárpát-medence Üszöggombái: 213, 1950.

Sporisorium ranunculi Lib. 1832. - Tuburcinia ranunculi (Lib.) Liro 1922.

Tuburcinia ranunculi-muricati Vienn.-Bourg. 1968.

Sori in leaves, petioles and stems, as conspicuous pustules of various shape and size, initially covered by the host epidermis which soon ruptures exposing the black, powdery mass of spore balls; later the leaves often become perforated. Spore balls globose to elongate or irregular, 12-28 $\times 14-$ 36(-44) $\mu \mathrm{m}$, composed of (0-)1-3(-5) spores and a few peripheral sterile cells or sterile cells may be lacking. Spores variable in shape and size, globose, ovoid, elongate or irregular, $10.5-15 \times 12-$ $22.5 \mu \mathrm{m}$, light to dark reddish brown, contents finely granular; wall c. $1 \mu \mathrm{m}$ thick, smooth. Sterile cells globose, ovoid to irregular, 6.5-10.5(-11) $\times 7-14.5(-16) \mu \mathrm{m}$, pale yellowish brown; wall 1-1.5 $\mu \mathrm{m}$ thick, smooth.

On Ranunculaceae: Ranunculus muricatus L., Gilan Prov., Anzali port.

77. Urocystis sorosporioides Körn. ex A.A. Fisch. Waldh., Aperçu Syst. Ustil.: 41, 1877b; Ann. Sci. Nat. Bot., Sér. 6, 4: 241, 1877a '1876'.

Urocystis sorosporioides Körn. in Fuckel 1876 (nom. nud.). - Tuburcinia sorosporioides (Körn. ex Fuckel) Liro 1922.

Sori as blister-like swellings, usually on the abaxial side of the leaves, or on the petioles and stems, initially lead-coloured and covered by the epidermis which ruptures to expose the black, powdery mass of spore balls. Spore balls globose, ovoid, elongate and irregular, 20-40 × 23-52(-60) $\mu \mathrm{m}$, dark reddish brown, composed of 1-10(-12) spores and a completely or almost completely investing layer of peripheral sterile cells. Spores globose, ovoid to elongate or subpolyhedral, irregular, 11$15(-17) \times 12-21 \mu \mathrm{m}$, dark reddish or olive-brown, smooth. Sterile cells variable in shape and size, 5-13 $\mu \mathrm{m}$ long, yellowish brown; wall smooth, collapsing with age (Fig. 61).

On Ranunculaceae: Thalictrum foetidum L., Mazenderan Prov., Chalous road, IRAN 7126 F.

On Thalictrum sultanabadense Stapf, Khorasan Prov., c. 40 km NE of Mashhad city, IRAN $7267 \mathrm{~F}$.

78. Urocystis tianschanica Golovin, Sredneaz. Gosud. Univ., N.S., Vyp. 14, Biol. Nauk, Kniga 5: $12,1950$.

Sori in leaves forming long striae, initially lead-coloured, covered by the epidermis which ruptures longitudinally disclosing the blackish brown, granular powdery mass of spore balls. Spore balls globose, ovoid, ellipsoidal, elongate or irregular, 16-36 × 20-40(-44) $\mu \mathrm{m}$, yellowish brown, composed of 1-4(-5) spores completely surrounded by sterile cells. Spores subglobose, ovoid, ellipsoidal or irregular, with one or two flattened sides, 9.5-14.5 $\times 12.5-19 \mu \mathrm{m}$, yellowish brown; wall even, c. $0.8 \mu \mathrm{m}$ thick. Sterile cells subglobose, ellipsoidal or irregular, 5-13 $\mu \mathrm{m}$ long, yellow; wall 1-1.5 $\mu \mathrm{m}$ thick, smooth (Fig. 62).

On Poaceae: Critesion violaceum (Boiss. \& Hohen.) Á. Löve (Hordeum violaceum Boiss. \& Hohen.), Tehran Prov., Alafchin, S slope of Damavand Mountain, 13.VI.1991, M. Abbasi, IRAN 8335 F, HUV 21988.

79. Urocystis tritici Körn., Hedwigia 16: 33, 1877.

Tuburcinia tritici (Körn.) Liro 1922.

Tuburcinia hispanica Syd. 1924. - Urocystis hispanica (Syd.) Zundel 1953.

Sori in leaves, sheaths and stems as long striae, initially lead-coloured, covered by the epidermis which ruptures longitudinally to expose the powdery, blackish brown mass of spore balls. Badly 
infected plants do not head. Spore balls subglobose, ellipsoidal to irregular, 16-30 × 20-40 $\mu \mathrm{m}$, composed of 1-3(-5) spores completely surrounded by sterile cells. Spores globose, ovoid, elongate or slightly irregular, 10-15 $\times 12-18(-22) \mu \mathrm{m}$, yellowish to reddish brown, smooth. Sterile cells subglobose to ovoid, 5-8 $\times 6-13 \mu \mathrm{m}$, pale yellowish brown; wall c. $1 \mu \mathrm{m}$ thick, smooth. Spore germination results in holobasidia apically producing (1-)2-4(-6) cylindrical basidiospores measuring 3-5 $\times 12-30 \mu \mathrm{m}$. Basidiospores germinate giving rise to slender infection hyphae and/or secondary sporidia (Fig. 63).

On Poaceae: Triticum aestivum L. and Triticum sp. Common in Iran.

80. Urocystis violae (Sowerby) A.A. Fisch. Waldh., Bull. Soc. Nat. Moscow 40: 258, 1867.

Granularia violae Sowerby 1809. - Polycystis violae (Sowerby) Berk. \& Broome 1850. Tuburcinia violae (Sowerby) Liro 1922.

Uredo (Caeoma) vesicaria J.S. Kaulf., in Kunze \& J.C. Schmidt 1817. - Erysibe arillata Wallr. var. violarum Wallr. 1833 (nom. nov. illegit. pro Uredo vesicaria). - Polycystis vesicaria (J.S. Kaulf.) Mont. 1856. - Urocystis vesicaria (J.S. Kaulf.) Brockm. 1863.

Sori usually in the petioles as fusiform swellings up to several $\mathrm{cm}$ long, often causing distortions, but also in stems, rootstocks, leaf veins or, infrequently, on the leaf blades as irregular, multilocular, blister-like swellings, rather persistently covered by the host tissues, which ruptures exposing the blackish brown, powdery spore mass. Spore balls globose, ovoid to elongate, irregular, 20-55 × 20$75 \mu \mathrm{m}$, reddish brown, composed of (1-)3-20 or more spores surrounded by a complete layer of sterile cells. Spores globose, ovoid, elongate, usually subpolyhedral, irregular, 9-15 × 11-20 $\mu \mathrm{m}$, medium reddish brown, smooth. Sterile cells variable in shape and size, 7-16 $\mu \mathrm{m}$ long, yellow, smooth, collapsing with age. Spore germination results in holobasidia producing apically 3-8 basidiospores on narrowly ovate lobes (Fig. 64).

On Violaceae: Viola sp., East Azarbaijan Prov., Arasbaran protected area, Kaleibar city towards Eskanlu city, IRAN $14201 \mathrm{~F}$.

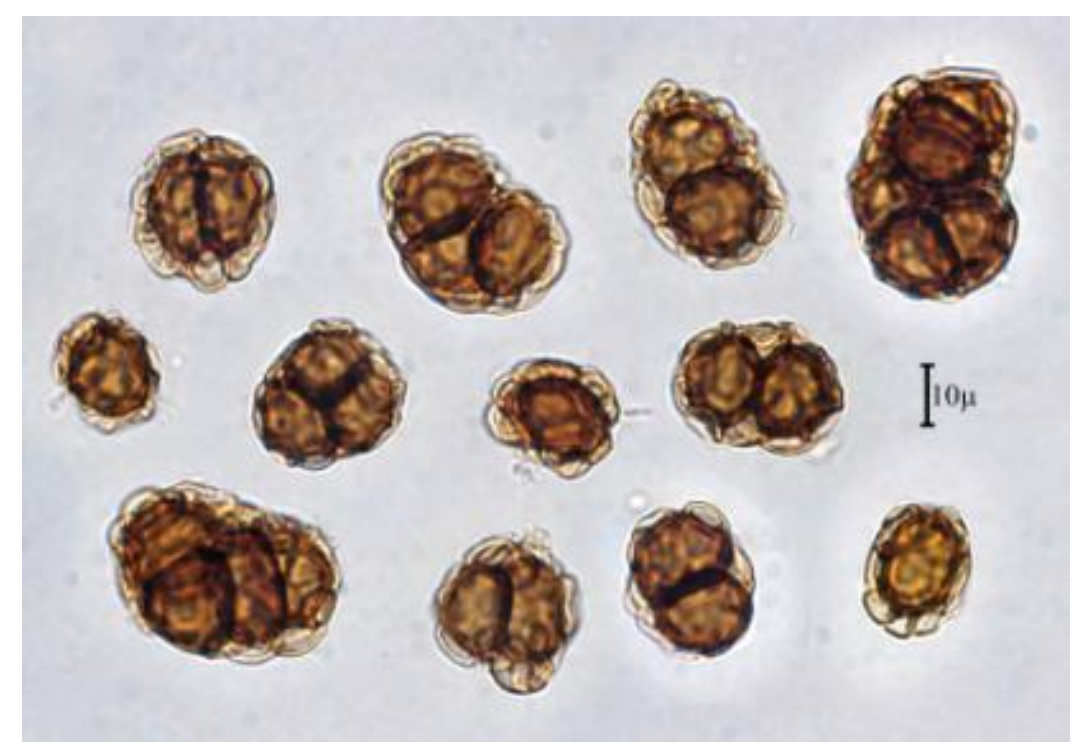

Fig.47 - Urocystis bolivarii on Lolium sp. (10339) 


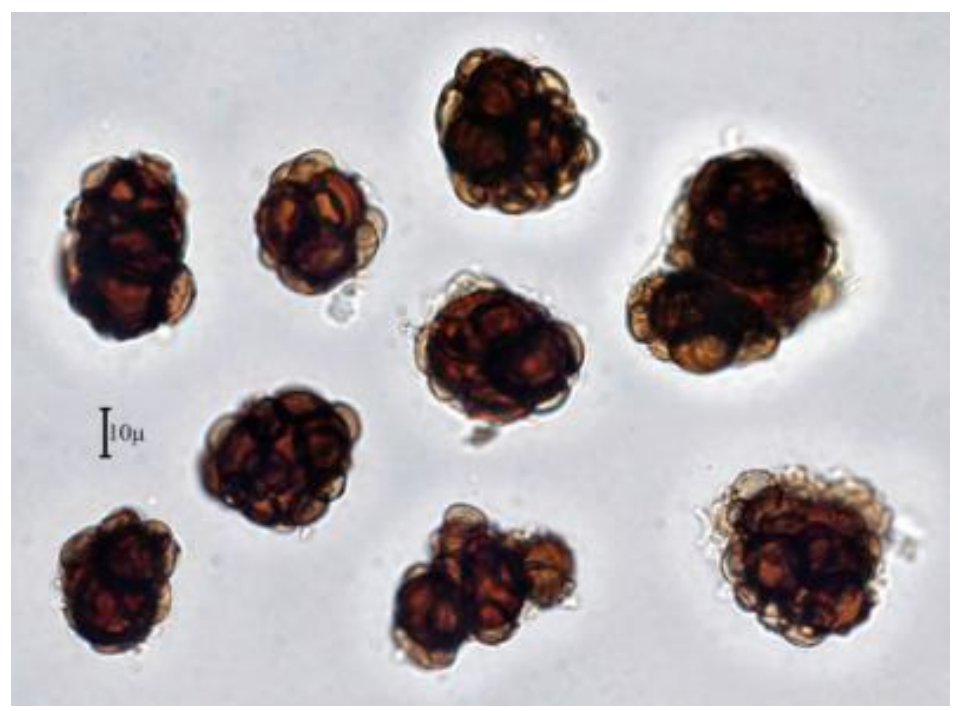

Fig.48 - Urocystis ceratocephali on Ceratocephalus falcatus (7238)

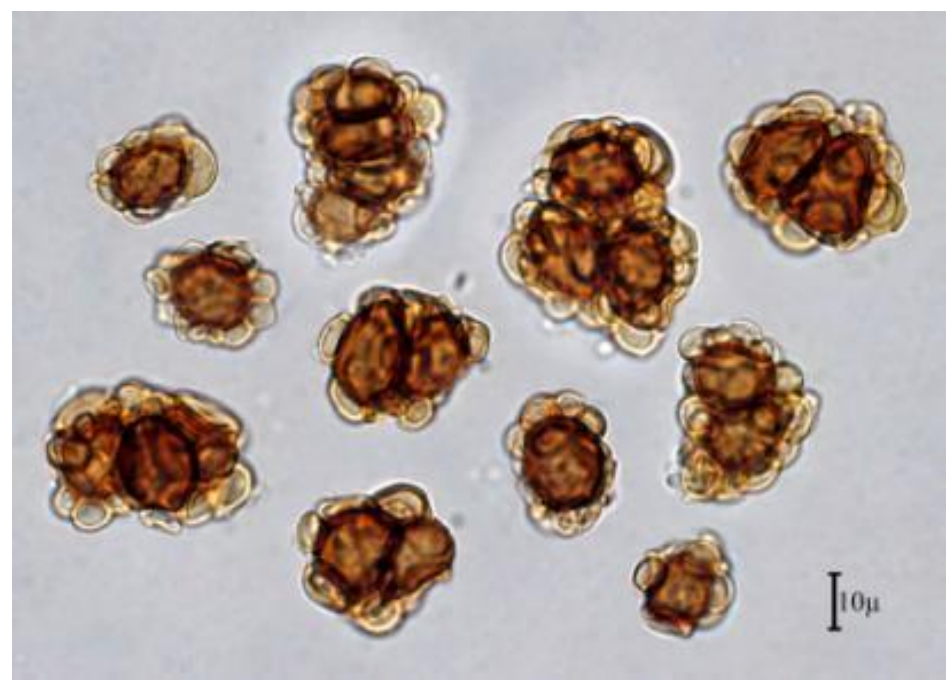

Fig.49 - Urocystis colchici on Colchicum persicum (8329)

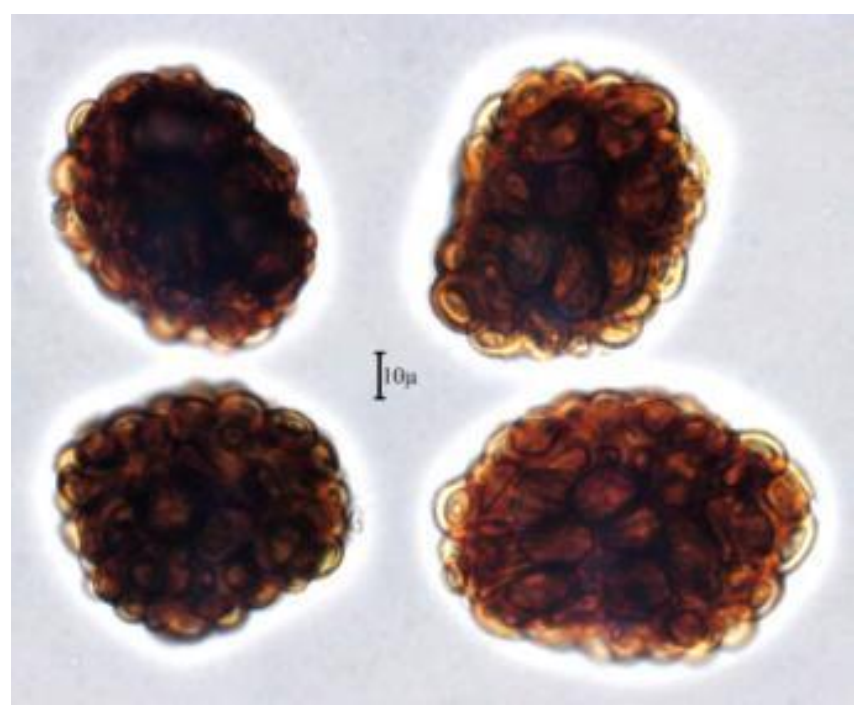

Fig.50 - Urocystis corsica on Stipa capensis (8296) 


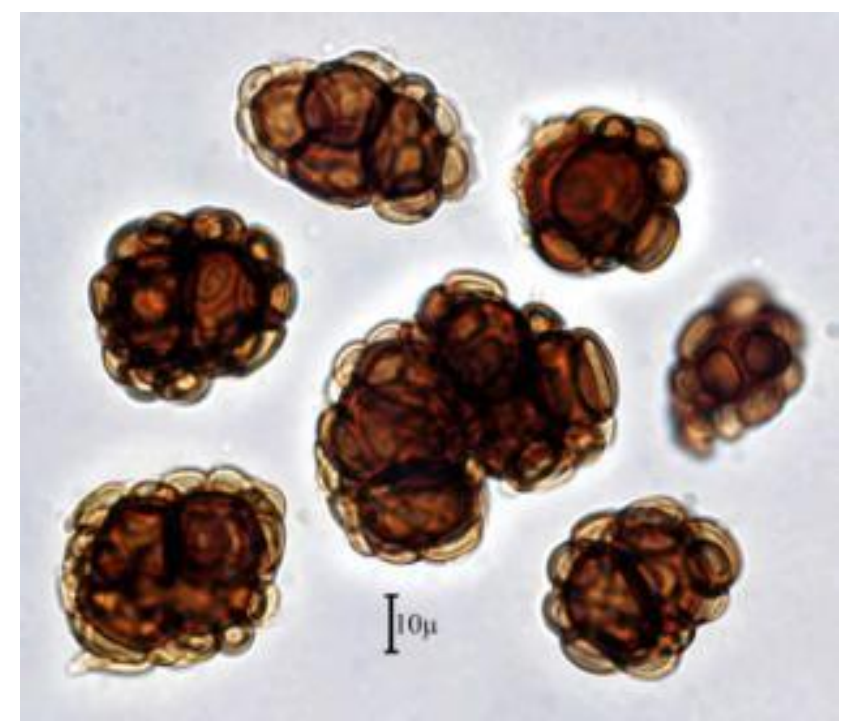

Fig.51 - Urocystis ficariae on Ranunculus ficaria (7237)

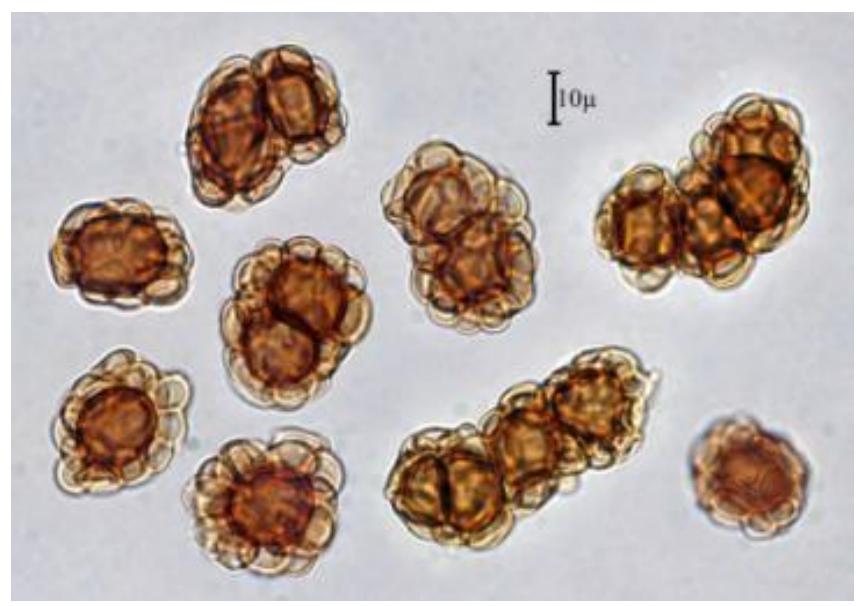

Fig.52 - Urocystis fischeri on Carex sp. (10908)

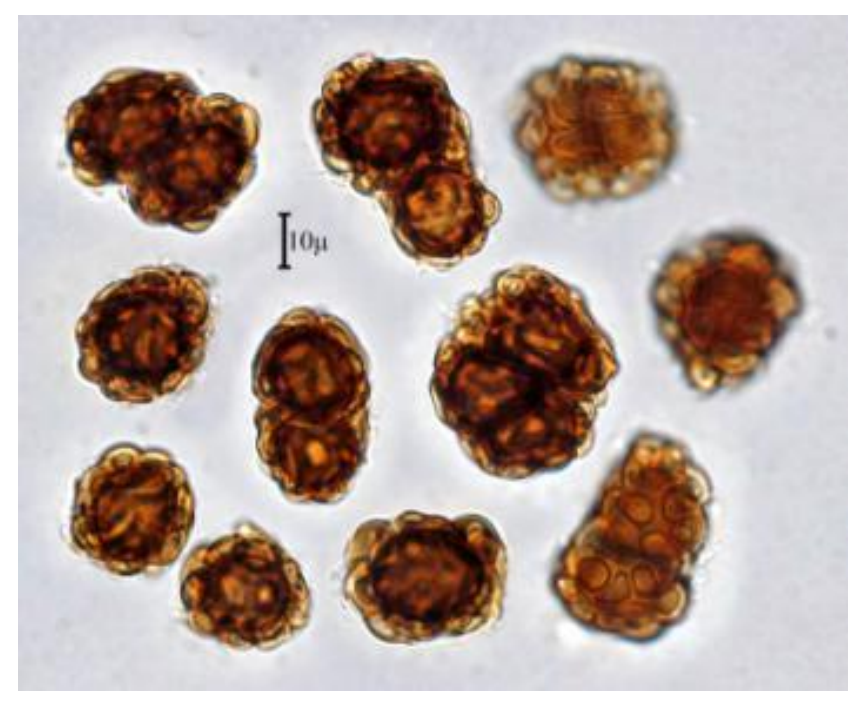

Fig.53 - Urocystis gladiolicola on Gladiolus segetum (8008) 


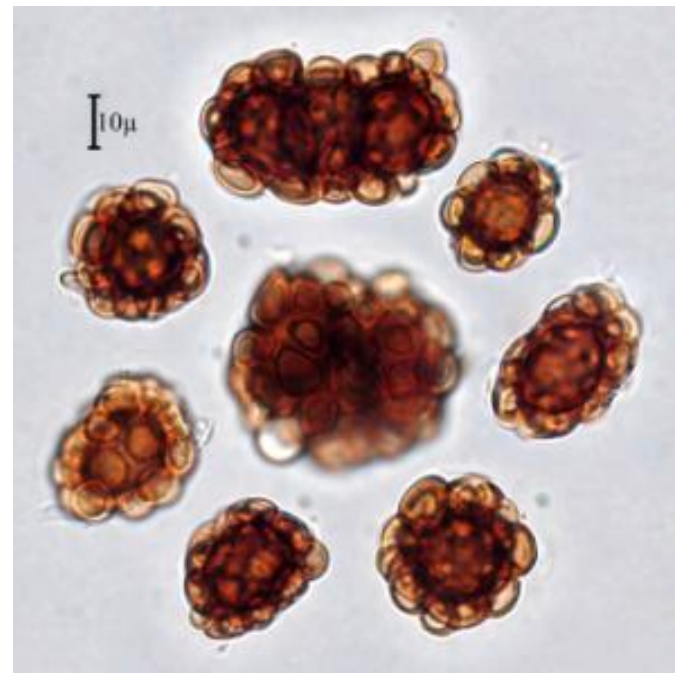

Fig.54 - Urocystis ixiolirii on Ixiolirion pallasii (10424)

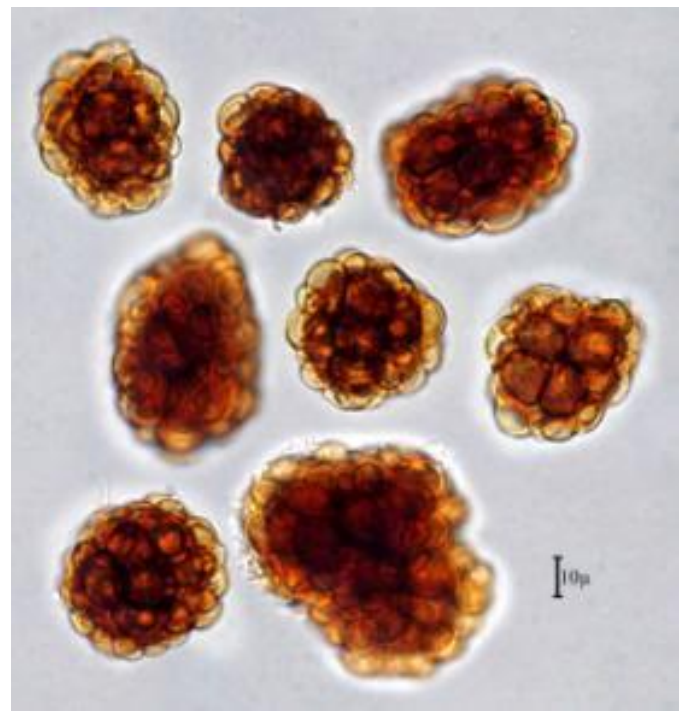

Fig.55 - Urocystis kmetiana on Viola occulta (7235)

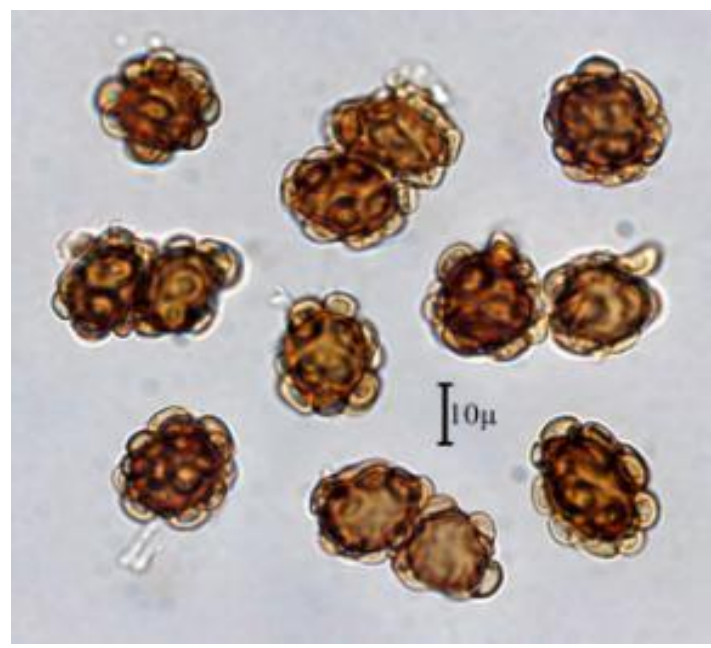

Fig.56 - Urocystis magica on Allium cepa (4391) 


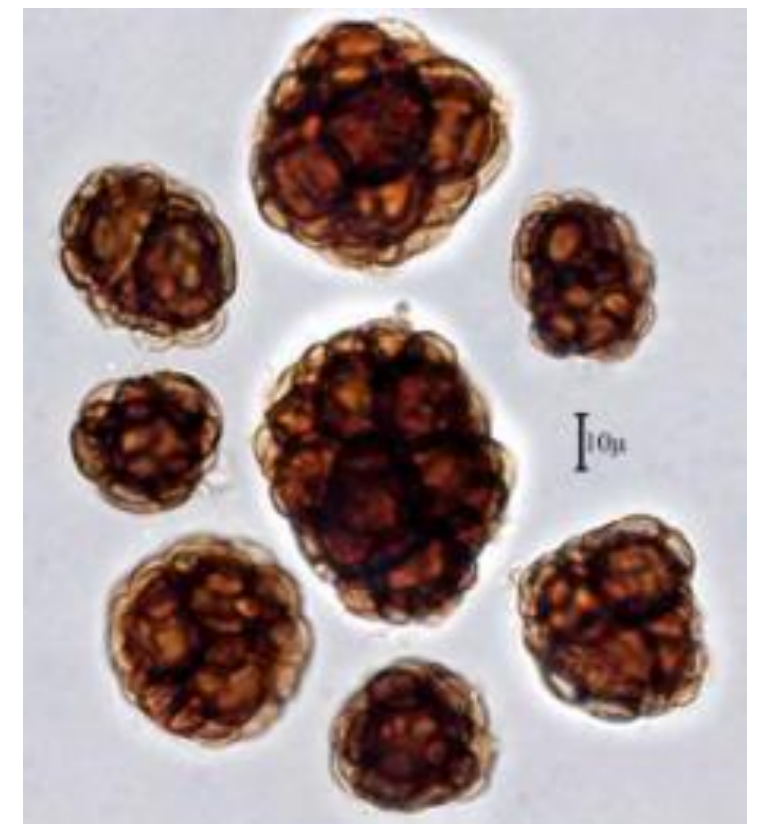

Fig.57 - Urocystis muscaridis on Muscari sp. (11799)

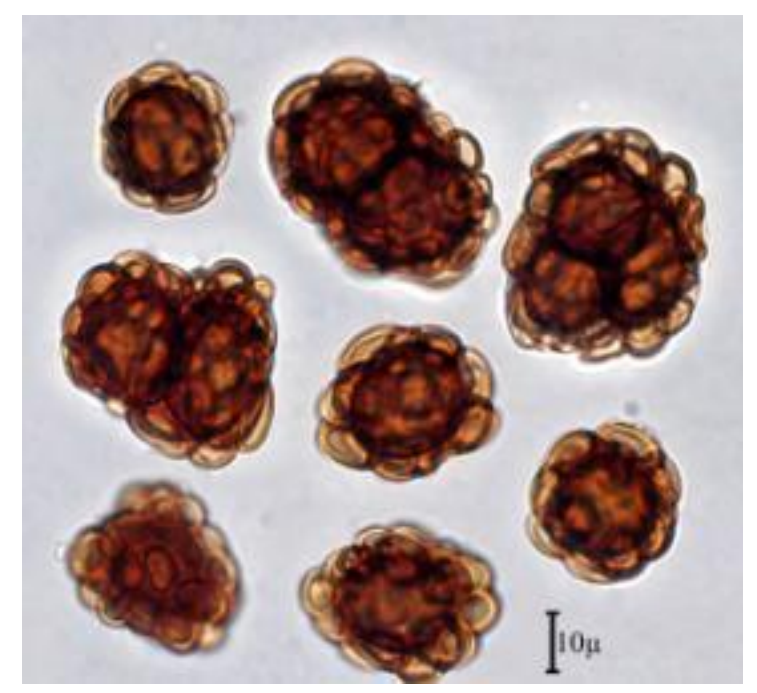

Fig.58 - Urocystis ornithogali on Ornithogalum kurdicum (11658)

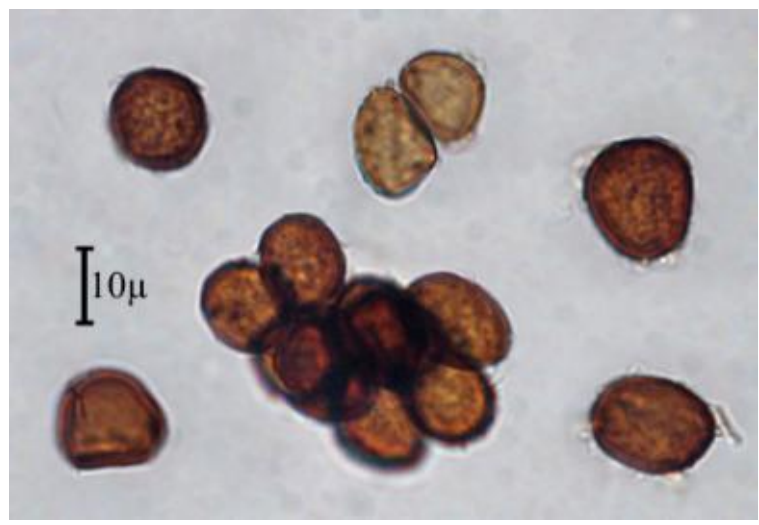

Fig.59 - Urocystis pedicularis on Pedicularis sibthorpii (8312) 


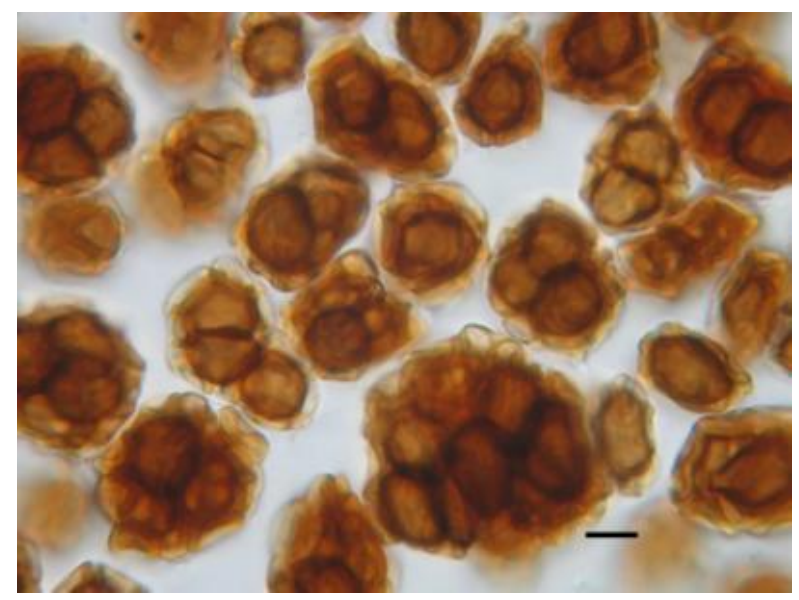

Fig.60 - Urocystis phalaridis on Phalaris sp. (10352), Bar=10 $\mu \mathrm{m}$

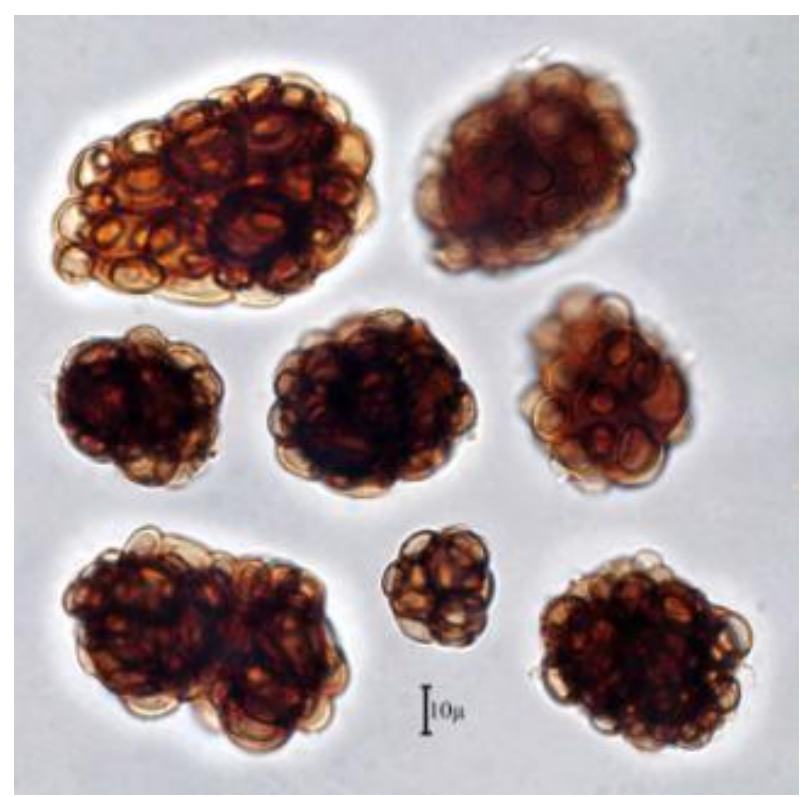

Fig.61 - Urocystis sorosporioides on Thalictrum sultanabadense (7267)

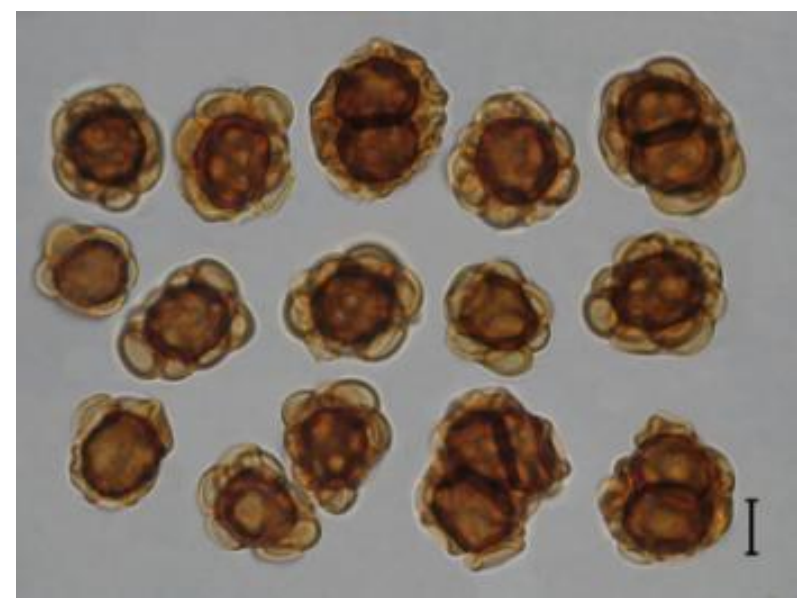

Fig.62 - Urocystis tianschanica on Critesion violaceum (8335), Bar=10 $\mu \mathrm{m}$ 


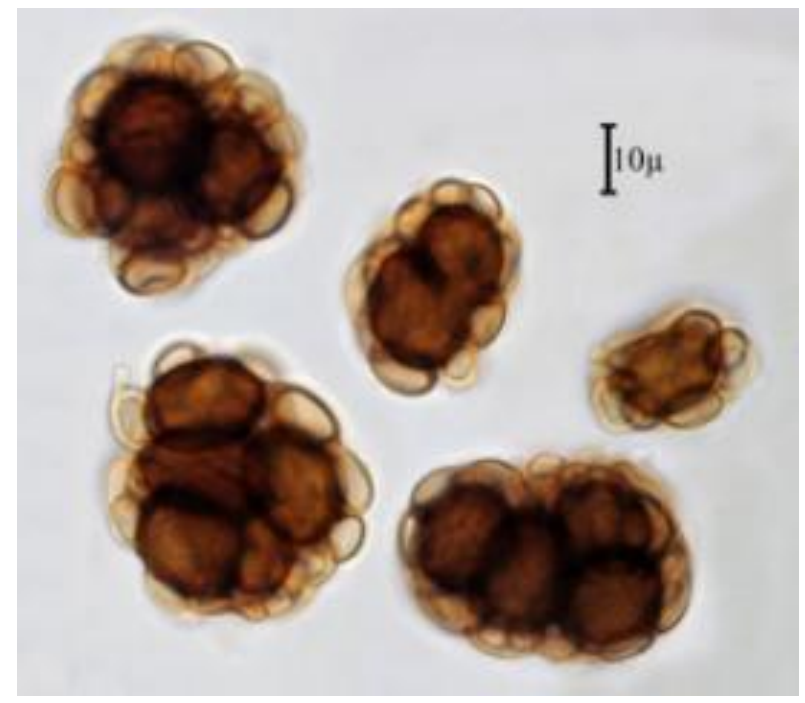

Fig.63 - Urocystis tritici on Triticum aestivum (13875)

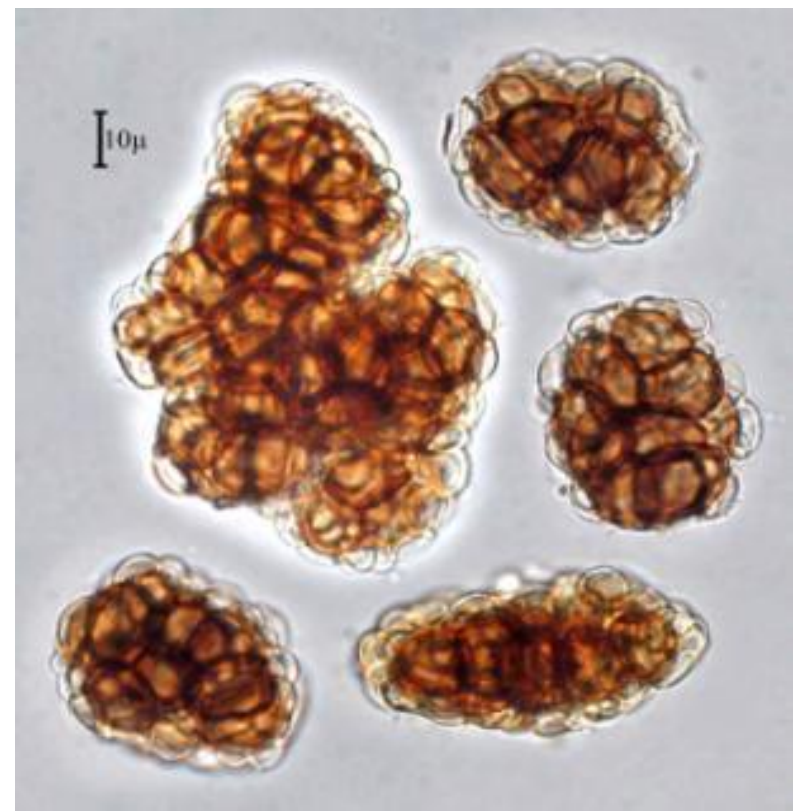

Fig.64 - Urocystis violae on Viola sp. (14201)

\section{Ustilago (Pers.) Roussel}

\section{Key to the Ustilago species based on host plant taxonomy}

1. On Aeluropus

U. aeluropodis

- Not on Aeluropus

2. On Aegilops U. avenae / U. tritici

- Not on Aegilops U. avenae

- Not on Avena 4

4. On Brachypodium. U. bromivora

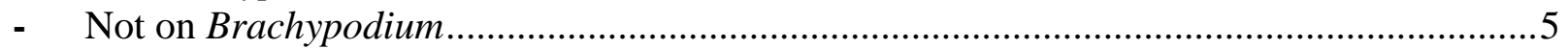

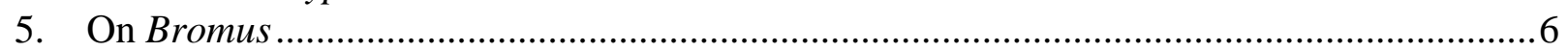

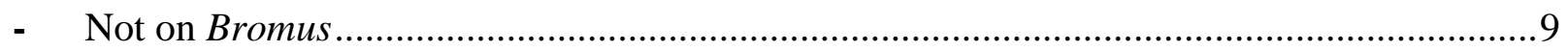

6. Sori around the stem and floral axis, often comprising also the basal part of the floral envelopes 
Sori not so

7. Sori in spikelets, on basal part of floral envelopes, bullate ............................. bromivora

- $\quad$ Sori in leafs and leaf sheaths as striae, rarely also in the inflorescence ............................ 8

8. Spores 9-15(-16) $\mu \mathrm{m}$ long, echinulate; sori in leaves

U. striiformis

- $\quad$ Spores 4-7 $\mu \mathrm{m}$ long, punctate-verruculose; sori also in distorted inflorescence

9. On Cynodon.

U. trebouxii

- Not on Cynodon

U. cynodontis

10. On Dactylis 10

- Not on Dactylis

U. striiformis

11. On Echinochloa 11

- Not on Echinochloa U. trichophora

12. On Eremopyrum .12

- Not on Eremopyrum

U. turcomanica

13. On Glyceria

- Not on Glyceria

U. filiformis

14. On Hordeum .14

- Not on Hordeum .15

15. Spores minutely echinulate .16 Spores smooth. U. hordei

16. On Poa U. nuda

- Not on Poa. U. striiformis

17. On Saccharum .17

- Not on Saccharum U. scitaminea

18. On Setaria 18

- Not on Setaria. U. crameri

19. On Taeniatherum 19

- $\quad$ Not on Taeniatherum U. phrygica

20. On Triticum .20

- On Zea U. tritici U. maydis

81. Ustilago aeluropodis (Trotter) Vánky, Publ. Herb. Ustilag. Vánky (HUV) 1: 11, 1985b. Sphacelotheca aeluropodis Trotter, in Sacc. \& Trotter 1913. - Crozalsiella aeluropodis (Trotter) Maire 1917.

Sorosporium aeluropodis S. Ahmad 1956 (as 'aeluropidis').

Sori on the tip of culms replacing the inflorescence, ovoid or fusiform, 1-2 cm long, invested by a rosette of leaf sheaths and young leaves, later rupturing laterally to expose the dark brown, powdery spore mass. Peridium, sterile cells and columellae lacking. Spores variable in shape and size, globose, subellipsoidal or ellipsoidal, often slightly flattened, 12-17 $\times 13.5-20 \mu \mathrm{m}$, golden brown; wall uniform, 0.5-1 $\mu \mathrm{m}$ thick, in LM sparsely to moderately densely, minutely punctate-verruculose; the verrucae do not or only slightly affect the spore profile, in SEM minutely verruculose-echinulate. Spore germination results in septate basidia and hyphae (Fig. 65).

On Poaceae: Aeluropus littoralis (Gouan) Parl., Khuzestan Prov., Ahvaz city.

On Aeluropus sp., Hormozgan Prov., Persian Gulf, Gheshm Island, IRAN 11342 F.

82. Ustilago avenae (Pers. : Pers.) Rostr., Overs. Kongel. Danske Vidensk. Selsk. Forh. Medlemmers Arbeider: 13, 1890 (March).

Uredo segetum Pers. var. avenae Pers. 1797. - Uredo segetum Pers. var. Uredo avenae Pers. : Pers. 1801. - Uredo carbo DC. var. avenae (Pers. : Pers.) DC. 1815. - Erysibe vera Wallr. var. avenae (Pers. : Pers.) Wallr. 1833. - Ustilago segetum var. avenae (Pers. : Pers.) Brunaud 1878. Ustilago segetum var. avenae (Pers. : Pers.) J.L. Jensen 1888 (comb. superfl.). - Ustilago avenae (Pers. : Pers.) J.L. Jensen, in Kellerm. \& Swingle 1890 (June). 
Erysibe vera Wallr. var. holci-avenacei Wallr. 1833 (nom. nud.). - Ustilago holci-avenacei (Wallr.) Cif. 1938 (comb. illegit.).

Ustilago perennans Rostr. 1890b (March).

Ustilago medians Biedenk. 1894.

Ustilago arrhenatheri Ferle 1912 (nom. nud.).

Ustilago decipiens ("Wallr.") Liro 1924.

Ustilago nigra Tapke 1932.

Ustilago aegilopsidis Picb. 1932.

Ustilago festucae Zundel 1933.

Ustilago haynaldiae Becer. 1970.

Sori in spikelets destroying the ovaries and more or less destroying the palea, lemma and glumes ("loose smut"). Spore mass first firm, then powdery, dark olivaceous to chocolate-brown. Spores globose, ovoid to elongate, $(4-) 5-7 \times 6-9 \mu \mathrm{m}$, yellowish brown to olive-brown, paler on one side, minutely echinulate, ornamentation usually more prominent on the paler side, in SEM with more or less densely spaced, rounded to subacute warts. Spore germination results in 4-celled basidia producing basidiospores on artificial media. Either compatible basidial cells or basidiospores fuse to form dikaryotic hyphae which infect seedlings. Infection is systemic (Fig. 66).

On Poaceae: Aegilops triuncialis (L.) Á. Löve, West Azerbaijan Prov., Orumieh-Salmas road.

On Avena barbata Pott ex Link, Golestan Prov., Golestan National Park, Dashte Kalpush area, IRAN $8315 \mathrm{~F}$.

On Avena fatua L., Khuzestan Prov., between Ahvaz city \& Andimeshk city, IRAN 7124 F; Golestan Prov., Gorgan city, c. 50 km SW of Maraveh-tappeh village, IRAN 7275 F.

On Avena ludoviciana Durieu, Yazd Prov., Meibod city, IRAN 13873 F.

On Avena sativa L., Khuzestan Prov., Dezful city, IRAN 4390 F; Yazd Prov., Meibod city, Shamsabad village, IRAN 14572 F.

On Avena sp., Kohgiluyeh va -Buyerahmad Prov., Nil Mt., IRAN 9852 F.; KohgiluyehBuyerahmad Prov., Dehdasht city; Kermanshah Prov., Sahneh city, IRAN 6635 F; Yazd Prov., Meibod city, IRAN 13874 F.

On Hordeum vulgare L., Khuzestan Prov., Ahvaz city; Khuzestan Prov., Andimeshk city; Khuzestan Prov., Masjed Soleiman city; Mazandaran Prov., Babolsar city; Mazandaran Prov., Kelardasht city; Gilan Prov., Rudbar city; Fars Prov., Firuzabad city, Ghirokarzin road, 15 km NW of Ghirokarzin city, IRAN 13193 F; Golestan Prov., Gorgan city, c. 35 km SE of Azadshahr city, IRAN $7276 \mathrm{~F}$.

83. Ustilago bromivora (Tul. \& C. Tul.) A.A. Fisch. Waldh., Bull. Soc. Nat. Moscou 40: 252, 1867. Ustilago carbo (DC.) Tul. \& C. Tul. var. vulgaris Tul. \& C. Tul. d bromivora Tul. \& C. Tul. 1847. Yenia bromivora (Tul. \& C. Tul.) Liou 1949.

Ustilago lorentziana Thüm. 1880.

Cintractia patagonica Cooke \& Massee, in Cooke 1889. - Ustilago patagonica (Cooke \& Massee)

Cif. 1928. - Ustilago patagonica (Cooke \& Massee) Lavrov 1951 (comb. superfl.).

Ustilago holwayi Dietel 1893.

Ustilago hordeicola Speg. 1909.

Ustilago brachypodii-distachyi Maire 1919.

Ustilago bromi-arvensis Liro 1924.

Ustilago bromi-mollis Liro 1924.

Ustilago jamalainenii Liro 1939.

Sorosporium maroccanum Unamuno 1940.

Ustilago grossheimii Uljan. 1950.

Ustilago zernae Uljan. 1950.

Ustilago compacta G.W. Fisch. 1952.

Sorosporium lavrovianum Smarods 1963.

Ustilago pospelovii Uljan. 1968. 
Sori in spikelets, usually destroying the basal parts of the glumes and even the ovaries; 4-10 mm in diam., bullate, initially agglutinated and covered by the epidermis but finally becoming dusty, black or blackish brown. Infection systemic (seedlings and older shoots are infected). Spores globose to subglobose, occasionally ovoid or irregularly polyhedral, 6.5-9.5 $\times 8-11 \mu \mathrm{m}$, olivaceous to reddish brown often with a darker equatorial band; wall even to slightly uneven, $1-1.5 \mu \mathrm{m}$ thick, in LM from finely to rather coarsely verrucose, more pronounced on the polar regions, in SEM coarsely verrucose. Spore germination: each spore usually forms two, two-celled basidia (occasionally one three-celled and one one-celled) on which terminal and lateral ovoid basidiospores are borne. Sometimes only one two-celled basidium is produced, the two cells conjugate giving rise to a dikaryotic hypha or, rarely to two basidiospores (Fig. 67).

On Poaceae: Brachypodium distachyon (L.) P. Beauv. (Trachynia distachya (L.) Link), Ilam Prov., Ilam city, IRAN 4389 F; Lorestan Prov., on the road of Khorramabad city to Andimeshk city; IRAN 8302 F; Kermanshah Prov., Rijab area, Shalan village, IRAN 14785 F, HUV 21827; Golestan Prov., $50 \mathrm{~km}$ SW of Maraveh tappeh village, IRAN 7248 F; Gilan Prov., at the lake of White River dam, IRAN $7247 \mathrm{~F}$.

On Bromus danthoniae (Desf.) Trin. ex C.A. Mey, East Azarbaijan Prov., between Azarshahr city and Ajabshir city; Sistan va -Baluchestan, Zabol city, IRAN 11698 F; Tehran Prov., Sorkhehesar National Park, E of the Park, Ala-Khancheshmeh spring, IRAN 6447 F; Hamadan Prov., Hamadan city.

On Bromus japonicus Thunb., Tehran Prov., Karaj city, Chalus road, $38 \mathrm{~km}$ N of Karaj city, at Tochal hotel, IRAN 11146 F.

On Bromus madritensis L., Gilan Prov., N of White River dam, IRAN 7244 F; Golestan Prov., Gorgan city, c. 35 km SE of Azadshahr city, IRAN 7242 F; Tehran Prov., Tehran city, Saadatabad area, IRAN 8089 F; Golestan Prov., Gonbad city, Beshelan village, IRAN 4388 F.

On Bromus rubens L., Bushehr Prov., Khormuj city, IRAN 9850 F; Tehran Prov., Tehran city to Ghom city, $55 \mathrm{~km}$ N of Ghom city, Emamzadeh Allali-Sallali area, IRAN $9849 \mathrm{~F}$.

On Bromus sterilis L., Tehran Prov., Campus of Iranian Research Institute of Plant Protection, IRAN 11189 F; Fars Prov., Shiraz city, Imam Hossein orchard, IRAN 13285 F; Tehran Prov., Jajroud city, Saeidabad village, beside Khojir National Park, IRAN 6452 F.

Bromus tectorum L., Tehran Prov., Tehran city, Darband area; Hamadan Prov., Malayer city; Tehran Prov., Karaj-Chalus road, at the Amirkabir dam, IRAN 11149 \& 7225 F; Tehran Prov., Karaj city, Baraghan village, IRAN 8767 F; Tehran Prov., Karaj-Chalus road, at the road of Kondor village, IRAN 10772 F; Tehran Prov., Karaj-Chalus road, Sira village, IRAN 7224 F; Tehran Prov., Karaj-Chalus road, Khor ski slope road, Jei \& Charan bifurcation, at the Gholgholak spring, IRAN 10898 F; Golestan Prov., Golestan National Park, Dashte Kalpush area. IRAN 8322 F; Khorasan Prov., Mashhad city, IRAN 8500 F; Tehran Prov., Karaj-Chalus road, on the road of Kalvan \& Sira villages, 11148 F; Tehran Prov., Tehran city, Central Elburz Mts., Darabad area, IRAN 7241 F; Tehran Prov., Chalus road, Amirkabir dam; Qazvin Prov., c. 50 km NW of Qazvin city, IRAN 7243 F; Tehran Prov., Sorkhe-hesar National Park, Ala-Khancheshmeh area, IRAN 1808 F; Tehran Prov., Parchin road, Khojir National Park, 18 km after guard post, IRAN 1823 F; Tehran Prov., Karaj city, Manzarieh area, Vahdat town, IRAN 11147 F; Chaharmahal va -Bakhtiari Prov., Shahre Kord city, Emamieh Garden, IRAN 11569 F; Tehran Prov., Jajroud protected area, Parchin road, 4 km after guard station, E. slopes of road, IRAN 6434 F; Tehran Prov., Jajroud protected area, Saeedabad village, W. slopes of Khojir National Park, IRAN 6391 F.

On Bromus sp., Hamadan Prov., Toyserkan city, IRAN 11754 F.

On Hordeum glaucum Steud., Ilam Prov., Dehloran city, IRAN 10534 F.

On Trachynia distachya see Brachypodium distachyon.

84. Ustilago crameri Körn., in Fuckel, Jahrb. Nassauischen Vereins Naturk. 27-28: 11, 1874, '1873-74'.

Sori in all spikelets of an inflorescence destroying the ovaries and the basal part of the inner floral envelopes which become bullate, while the upper parts of the envelopes and the two glumes are 
normally developed. Sori initially covered by a green, yellow or silvery peridium of host origin which ruptures irregularly exposing the dark brown, powdery mass of spores. Spores variable in shape, subglobose to elongate, slightly bent, irregular, subpolyhedral, pyriform, lemon- or dropshaped, sometimes with an acute tip, (6-)7-9.5 × 8-12(-15) $\mu \mathrm{m}$, medium dark reddish brown; wall even, c. $0.5 \mu \mathrm{m}$ thick, smooth; in SEM smooth or with sparsely situated, low warts.

On Poaceae: Setaria italica (L.) P. Beauv., Mazandaran Prov., Kojur; Mazandaran Prov., Nur; Mazandaran Prov., Kelardasht.

On Setaria viridis (L.) P. Beauv., Tehran Prov., Karaj city; Tehran Prov., Tehran city.

85. Ustilago cynodontis (Henn.) Henn., Bull. Herb. Boissier 1: 114, 1893.

Ustilago segetum ("Bulliard") Ditmar var. cynodontis Henn. 1892.

Uredo digitariae Rabenh. 1847 (later homonym, not Kunze 1830). - Ustilago pallida Körn. 1877b

(March), nom. nov.

Ustilago carbo (DC.) Tul. \& C. Tul. [var.] cynodontis Pass. 1871. - Ustilago cynodontis (Pass.)

Curzi, in Curzi \& Barbaini 1927. (later homonym, not (Henn.) Henn.).

Ustilago nebrodensis Gonz. Frag. 1919.

Sporisorium agropyri Bag \& D.K. Agarwal 2001.

Sori in inflorescence destroying the spikelets and covering the spikes with a dark brown, dusty spore mass; sometimes the infection is localised to the basal parts of the inflorescence, usually with abortive spikelets in its distal parts; young sori often more or less hidden by enveloping leaf sheaths. Infection systemic. Spores globose to subglobose, 5.5-8 $\times 6-8(-8.5) \mu \mathrm{m}$, yellowish brown to light olive-brown, smooth, in SEM with dense, minute warts. Young spores often in readily separable chains, connected by small, hyaline hyphal remnants. Spore germination results in 4-celled basidia (in $3+1$ arrangement) producing successively, lateral and terminal, ovoid to long ellipsoidal basidiospores. Basidiospores bud or germinate by hyphae on which lateral or terminal aerial sporidial systems develop (Fig. 68).

On Poaceae: Cynodon dactylon (L.) Pers. Common in Iran.

On Cynodon sp., Golestan Prov., Minoudasht city, Nil Mt., IRAN 4378 F.

86. Ustilago filiformis (Schrank) Rostr., Festskr. Bot. Foren. Kjöbenhavn 1890: 136, 1890a.

Lycoperdon filiforme Schrank 1793.

Uredo longissima Sowerby 1799. - Caeoma longissimum (Sowerby) Schltdl. 1824. - Erysibe longissima (Sowerby) Wallr. 1833. - Ustilago longissima (Sowerby) Meyen 1841. - Ustilago longissima (Sowerby) Tul. \& C. Tul. 1847 (comb. superfl.). - Yenia longissima (Sowerby) Liou 1949.

Uredo (Ustilago) fuscovirens Ces., in Rabenh. 1850, 1851.

Ustilago longissima var. dubiosa Liro 1924. - Ustilago dubiosa (Liro) Liro 1938 (later homonym, not U. dubiosa Speg. 1881). - Ustilago agropyrina Lavrov 1936 (nom. nov., not $U$. dubiosa Speg.). - Ustilago liroae Zundel 1953 (not U. dubiosa Speg.).

Ustilago glyceriae Cif. 1931.

Entyloma peninsulae Crowell 1942.

Sori in leaves and sheaths as long striae between the veins, initially lead-coloured, covered by the epidermis which soon ruptures longitudinally and the pale brown, powdery spore mass becomes scattered. Infection systemic, infected plants usually do not flower. Spores globose, subglobose or ovoid, 4-5 $\times 4-6(-7) \mu \mathrm{m}$, pale olive-brown; wall c. $0.5 \mu \mathrm{m}$ thick, very finely punctate, spore profile smooth or very finely wavy, in SEM coarsely low verrucose to echinulate. Spore germination: meiosis occurs in the germinating spore. The developing basidium separates into a three- and a onecelled piece both of which elongate considerably and also increase in width. The basidiospores developed from the various bits of the basidium are long and narrow. On liberation they undergo considerable elongation and give rise to daughter cells in lateral or terminal positions (Fig. 69).

On Poaceae: Glyceria plicata Fr., Fars Prov., Arzhan plain, toward Chero waterfall, Juk spring, IRAN 12787 \& 12822 F; Tehran Prov., Firuzkuh city toward Tehran city, 118 km E of 
Tehran, Emamzadeh Esmail, on river-bank, IRAN 12848 F; East Azarbaijan Prov., Arasbaran protected area, Kaleibar city toward Makidi village, IRAN $14216 \mathrm{~F}$.

87. Ustilago hordei (Pers. : Pers.) Lagerh., Mitt. Bad. Bot. Vereins 1889: 70, 1889 (March).

Uredo segetum Pers. var. hordei Pers. 1797. - Uredo segetum Pers. var. Uredo hordei Pers. : Pers. 1801. - Uredo carbo DC. var. hordei (Pers. : Pers.) DC: 1815. - Erysibe vera var. hordei (Pers. : Pers.) Wallr. 1833. - Ustilago segetum (Pers.) Roussel var. hordei (Pers. : Pers.) Rabenh. 1856. Ustilago segetum (Pers.) Roussel var. hordei (Pers. : Pers.) Brunaud 1878. (comb. superfl.).

Reticularia segetum Bull. 1791 (pro parte, as includes all covered- and loose smuts of cereals, nom. rejic.). - Uredo segetum ("Bull.") Pers. 1797, p.p. - Ustilago segetum ("Bull.") Roussel 1806, p.p.

Ustilago segetum (Pers.) Roussel var. hordei (as 'Hordii') f. tecta Rostr. \& J.L. Jensen, in Jensen 1888 (nom. nud.). - Ustilago jensenii Rostr. 1890b (March).

Ustilago avenae (Pers. : Pers.) Rostr. var. levis Kellerm. \& Swingle 1890. - Ustilago levis (Kellerm. \& Swingle) Magnus 1894.

Ustilago kolleri Wille 1893.

Ustilago dura Appel \& Gassner 1907.

Ustilago arrhenatheri Schellenb. 1915.

Ustilago rostrupii Kitunen 1922.

Sori in all spikelets of an inflorescence as a blackish brown, adherent spore mass, initially covered by a membrane of host tissue origin ("covered smut"), later exposed to various degrees depending on the extent of destruction of the spikelet parts. Spores globose, subglobose to ovoid, 5-9(-10) $\mu \mathrm{m}$ in diam., paler coloured on one side, smooth. Spore germination results in 4-celled basidia (often of the $3+1$ pattern) producing basidiospores on artificial media; dikaryons formed between either compatible basidial cells or basidiospores infect seedlings (Fig. 70).

On Poaceae: Hordeum leporinum Link, Ghom Prov., Ghom city; Guilan Prov., Manjil city.

On Hordeum vulgare L. Common in Iran.

On Hordeum sp. Numerous collections.

88. Ustilago maydis (DC.) Corda, Icones Fungorum Hucusque Cognitorum 5: 3, 1842.

Uredo maydis DC. 1815. - Erysibe maydis (DC.) Wallr. 1833. - Mycosarcoma maydis (DC.) Bref. 1912.

Lycoperdon zeae Beckm. 1768.

Uredo segetum Pers. var. mays-zeae DC. 1805. - Uredo segetum Pers. var. Uredo zea-mays DC. in Poiret 1808 (var. nov. illegit.). - Ustilago zeae-mays (DC.) G. Winter, in Rabenh. 1881 (comb. illegit.). - Ustilago mays-zeae (DC.) Magnus 1896a('1895').

Uredo [subgen.] Ustilago zeae Schwein. 1822.

Caeoma zeae Link 1825 (nom. illegit. as it includes Uredo maydis). - Ustilago zeae (Link) Unger 1836 (comb. illegit.).

Ustilago euchlaenae Arcang. 1882.

Sori in stems, leaves or inflorescence (both male and female ones) as pustules or irregular galls of considerable size, initially covered by a thin, greyish silvery, later brown, smooth membrane which ruptures irregularly to expose the medium to dark brown, powdery spore mass. Spores globose, subglobose, ovoid to sometimes elongate or slightly irregular, 7-11 $\times 7-13 \mu \mathrm{m}$, light olive-brown; wall c. $0.5 \mu \mathrm{m}$ thick, finely, rather densely echinulate. Spore germination results in a 4-celled basidia laterally and terminally bearing basidiospores. Often, the upper half of the young basidium separates from the lower half by a fragmentation zone. Mycelium mostly intracellular (Fig. 71).

On Poaceae: Zea mays L. Not uncommon.

89. Ustilago nuda (C.N. Jensen) Kellerm. \& Swingle, An. Rep. Kans. Agr. Exp. Sta. 2: 277, 1890 (June). 
Ustilago segetum (Pers.) Roussel var. hordei (as 'Hordii') Rostr. \& C.N. Jensen f. nuda C.N. Jensen 1888 (nom. nud.). - Ustilago nuda (C.N. Jensen) Rostr. 1889 (nom. nud.).

Ustilago nuda var. foliicola Trotter, in Sacc. \& Trotter 1913.

Sori in all spikelets of an inflorescence, dark olive-brown, dusty ("loose smut"), usually destroying all floral parts leaving behind only the naked rachis; occasionally also on leaves, sheaths and culms as striae. Spores globose to subglobose or ovoid, 5-7 $\times 6-9 \mu \mathrm{m}$ in diam., olive-brown, paler on one side, minutely echinulate. Spore germination results in septate, 4-celled basidia. On artificial media and in nature fusion between compatible basidial cells gives rise to dikaryotic hyphae which infect the embryo resulting in a systemic disease Hyphae both intercellular and intracellular (Fig. 72).

On Poaceae: Hordeum vulgare L. Common in Iran.

90. Ustilago pamirica Golovin, Sredneaz. Gosud. Univ., N.S., Vyp. XIV, Biol. Nauk., Kniga 5: 11, 1950.

Ustilago pamirica Golovin, in Gutner 1941 (nom. inval.).

Sori around the stem and floral axis, often comprising also the basal part of the floral envelopes, initially covered by a silvery membrane which ruptures exposing the blackish brown, powdery mass of spores. Spores globose, subglobose to ovoid, 11-13(-14) $\times 11-15(-16) \mu \mathrm{m}$, reddish brown; wall 1-1.5 $\mu \mathrm{m}$ thick, densely, coarsely verrucose. Spore germination results in a whorl of four, ellipsoidal, one-celled germ tubes (basidia), producing apical chains of long-ellipsoidal basidiospores and later also lateral basidiospores (Fig. 73).

On Poaceae: Bromus gracillimus Bunge, Esfahan Prov., between Shahreza city and Semirom city, IRAN 7251 F, HUV 15080, also in Vánky, Ust. exs. no. 789.

91. Ustilago phrygica Magnus, Bull. Herb. Boissier 3: 574, 1903.

Ustilago hordei-criniti Barbarin, in Zaprom. 1928.

Ustilago tuberculata Golovin 1952.

Ustilago mesatlantica Malençon \& Massenot, in Guyot, Malençon \& Massenot 1969.

Sori usually destroying all spikelets in a spike, leaving intact only the more or less stunted and deformed awns, confluent, cylindrical, slightly bullate, subepidermal, lead-coloured, for a long time remaining covered by the epidermis which ruptures exposing the blackish brown, powdery spore mass. Infection at seedling stage. Spores globose, ellipsoidal to slightly irregular, (6-)7-8 $\times 7-10(-$ 11) $\mu \mathrm{m}$, medium olive-brown; wall uniformly $1-1.5 \mu \mathrm{m}$ thick, in LM moderately densely, somewhat irregularly verrucose or low tuberculate, in SEM densely, minutely tuberculate. Spore germination: A spore produces 1-2, one-, two- or three-septate basidia bearing ovoid basidiospores which bud abundantly (Fig. 74).

On Poaceae: Taeniatherum caput-medusae (L.) Nevski, Tehran Prov., Jajroud protected area; Parchin road, toward Parchin, $17 \mathrm{~km}$ S of guard station, IRAN 6386 F; Tehran Prov., Parchin road, $13 \mathrm{~km}$ S. of guard station, IRAN 1791 F; Tehran Prov., Karaj-Chalus road, near Amirkabir dam, road side, IRAN 10897 F; Tehran Prov., 20 km NE of Karaj city, at "Amir-Kabir" barrage, IRAN 7245 F; Tehran Prov., Northern part of Khojir national Park, IRAN 1761 F.

On Taeniatherum crinitum (Schreb.) Nevski, Fars Prov., at Daryun village, IRAN 13547.

On Taeniatherum sp., Tehran Prov., Khojir National Park, IRAN 1764 F.

92. Ustilago scitaminea Syd., Ann. Mycol. 22: 281, 1924 (nom. cons.).

Sporisorium scitamineum (Syd.) M. Piepenbr., M. Stoll \& Oberw. 2002.

Ustilago amadelpha Syd., P. Syd. \& E.J. Butler 1912 (nom. rejic.).

Sphacelotheca miscanthi W.Y. Yen 1937a. - Sporisorium miscanthi (W.Y. Yen) L. Guo 1990.

Ustilago scitaminea Syd. var. sacchari-barberi Mundk. 1939b.

Ustilago scitaminea Syd. var. sacchari -officinarum Mundk. 1939b.

Sori in floral stems which are transformed into long, flagelliform, often curved bodies; basal part of the sori concealed by leaf sheaths, distal part free and tapering, initially covered by a silvery peridium of host tissue which flakes away exposing the blackish brown, dusty mass of spores mixed 
with irregular groups of sterile cells. Spores globose, subglobose to subovoid, 5.5-7.5 $\times 6.5-8(-10)$ $\mu \mathrm{m}$, reddish brown; wall uniform, 0.5-0.8 $\mu \mathrm{m}$ thick, from almost smooth, finely and sparsely punctate-verruculose to sparsely or moderately densely echinulate. Sterile cells variable in shape and size, larger than the spores (8-23 $\mu \mathrm{m}$ in diam.), yellow or pale yellowish brown, smooth. Spore germination results in 4-celled basidia (often of $3+1$ pattern; the $4^{\text {th }}$ basidial cell remaining within the spore) on which laterally and terminally, ovoid to long ellipsoidal basidiospores are produced. Basidiospores bud or fuse in pairs, giving rise to dikaryotic hyphae. Under natural conditions, on sugarcane (and on certain media), basidial cells conjugate and produce septate, dikaryotic infection hyphae (Fig. 75).

On Poaceae: Saccharum officinarum L., Khuzestan Prov., Dezful city, Haft-tappeh Sugar Cane Company, IRAN 4343 F; Mazandaran Prov., Sari city, Dashte Naz area, IRAN 10712 F.

93. Ustilago striiformis (Westend.) Niessl, Hedwigia 15: 1, 1876, s. lat.

Uredo striiformis Westend. 1851 (as 'striaeformis'). - Tilletia striiformis (Westend.) Sacc. 1877. Tilletia striiformis (Westend.) G. Winter 1881 (comb. superfl.).

Ustilago salweyi Berk. \& Broome 1850 (as 'salveii'; nom. ambig.). - Uredo salweyi (Berk. \& Broome) Oudem. 1866. - Tilletia salweyi (Berk. \& Broome) P. Karst. 1884.

Uredo (Ustilago) longissima Sowerby var. megalospora Riess, in Rabenh. 1854. - Ustilago megalospora (Riess) Cif. 1933.

Tilletia debaryana A.A. Fisch. Waldh., in Rabenh. 1866 (as 'de Baryana').

Tilletia milii Fuckel 1870. - Ustilago milii (Fuckel) Liro 1924.

Ustilago denotarisii A.A. Fisch. Waldh. 1877c (as 'de Notarisii').

Tilletia alopecurivora Ule 1884. - Ustilago alopecurivora (Ule) Liro 1924.

Tilletia avenae Ule 1884. - Ustilago scaura Liro 1924 (not U. avenae (Pers. : Pers.) Rostr 1890b).

Tilletia brizae Ule 1884. - Ustilago brizae (Ule) Liro 1924.

Ustilago washingtoniana Ellis \& Everh. 1895.

Ustilago poarum McAlpine 1895.

Tilletia? airae-caespitosae Lindr. [Liro] 1904. - Ustilago airae-caespitosae (Lindr.) Liro 1924.

Tilletia corcontica Bubák 1912. - Ustilago corcontica (Bubák) Liro 1924.

Ustilago festucarum Liro 1924.

Ustilago bromina Syd. 1924.

Ustilago striaeformis f. agrostis-palustris W.H. Davis 1928 (nom. nud. et eventuale). - Ustilago agrostis-palustris W.H. Davis ex Cif. 1931.

Ustilago striaeformis f. phlei-pratensis W.H. Davis 1928 (nom. nud. et eventuale). - Ustilago phleipratensis W.H. Davis ex Cif. 1931.

Ustilago striiformis f. hierochloae-odoratae Săvul. \& Rayss 1932. - Ustilago hierochloae-odoratae (Săvul. \& Rayss) Cif. 1938.

Ustilago johnstonii Cif. 1933.

Ustilago kairamoi Liro 1939.

Ustilago clintoniana W.H. Davis 1935 (later homonym and nom. nud.; not U. clintoniana Ciferri 1928).

Ustilago poae-annuae W.H. Davis 1935 (nom. nud. et eventuale).

Ustilago poae-pratensis W.H. Davis 1935 (nom. nud. et eventuale).

Ustilago poae S. Ito 1936 (nom. inval.).

Ustilago jaczevskyana Lavrov var. jaczevskyana (as 'var. typica Lavrov') 1936.

Ustilago jaczevskyana var. sibirica Lavrov 1936.

Ustilago triseti Liro 1939.

Ustilago anthoxanthi Liro 1939.

Ustilago linearis (Berk. \& Broome) Petch 1924.

Ustilago loliicola Cif. 1938.

Ustilago taenia Cif. 1938.

Ustilago poae-bulbosae Săvul. 1951. 
Ustilago poae-nemoralis Vienn.-Bourg. 1951.

Ustilago duriusculae L. Guo 2006b.

Sori in leaves, leaf sheaths and culms as streaks parallel with the veins, occasionally by fusion covering most of the leaf surface, rarely also in the inflorescence and exceptionally in the ovaries, initially covered by the epidermis which soon ruptures, the dark brown, semi-powdery to powdery spore mass becoming scattered and the leaf-blades often shred. Infection systemic. The fungus perennates as mycelium in crowns, stolons or other organs. Spores globose, ovoid to irregular, 8$12(-13) \times 9-15(-16) \mu \mathrm{m}$, light olive-brown, finely to prominently echinulate, profile serrulate. Spore germination results in septate basidia which produce lateral branches or, under other conditions, basidiospores (Fig. 76).

On Poaceae: Bromus stenostachyus Boiss., Mazandaran Prov., Kelardasht city, Roodbarak village, IRAN $8347 \mathrm{~F}$.

On Dactylis glomerata L., Tehran Prov., Fasham city; Tehran Prov., Darakeh village, Palangchal area, IRAN 8331 F; Tehran Prov., Afjeh village, IRAN 8068 F.

On Poa annua L., Chahar-Mahal va -Bakhtiari Prov., Zard-kuh Mt., IRAN 8033 F.

On Poa trivialis L., Khorasan-e Razavi Prov., 45 km W of Mashhad city, Abardeh-Olia village, IRAN $7286 \mathrm{~F}$.

On Poa sp., Tehran Prov., Karaj-Chalus road, on the road Shahrestanak, 7 km after bifurcation towards Shahrestanak village, in orchard, IRAN 10896.

On unknown Poaceae, Tehran Prov., Karaj-Chalus road, Azadbar village, IRAN 10499 F.

Remark. Report of U. striiformis on Scirpus pumilus Vahl from Tehran Prov., Darakeh Valley, Palangchal area, Espiu spring, IRAN $8311 \mathrm{~F}$ (Ershad 2001: 196) is erroneous. The host plant is a Poaceae.

94. Ustilago trebouxii Syd. \& P. Syd., Ann. Mycol. 10: 214, 1912.

Ustilago sitanii G.W. Fisch. 1938.

Ustilago kazachstanica Gutner \& Sergeeva 1941.

Ustilago underwoodii Zundel 1942.

Ustilago helictotrichi Schmied., in Schmied. \& Puncag 1966.

Sori in upper leaves and leaf sheaths as long, dark brown, dusty striae, but also in distorted inflorescence, more or less destroying them. Spore mass exposed early. Spores globose, subglobose, ovoid to slightly irregular, (3.5-)4-5.5 $\times 4-7 \mu \mathrm{m}$, light olive-brown tending to be paler on one side, apparently smooth to finely, sparsely punctate-verruculose. Spore germination results in long, 4celled basidia, producing navicular basidiospores on well-developed sterigmata (Fig. 77).

On Poaceae: Bromus tomentellus Boiss., Chaharmahal va -Bakhtiari Prov., Farsan city, Deh Cheshmeh village, Pirghar spring, IRAN 8088 F.

On unknown Poaceae, Khorashan-e Shomali Prov., 13 km W of Bojnurd city, Badranlu Pass, IRAN $7274 \mathrm{~F}$.

95. Ustilago trichophora (Link) Kunze ex Körn., Hedwigia 16: 36, 1877.

Caeoma trichophorum Link 1825.

Ustilago sphaerogena Burrill, in Sacc. 1888. - Cintractia sphaerogena (Burrill) H.H. Hume 1902.

Ustilago crus-galli Tracy \& Earle 1895. - Cintractia crus-galli (Tracy \& Earle) Magnus 1896b. Ustilago trichophora var. crus-galli (Tracy \& Earle) Lavrov 1936.

Ustilago panici-frumentacei Bref. 1895. - Ustilago trichophora var. pacifica Lavrov 1936. Ustilago trichophora (Link) Kunze ex Körn. var. panici-frumentacei (Bref.) Mundk. 1943.

Cintractia seymouriana Magnus $1896 \mathrm{~b}$.

Ustilago globigena Speg. 1899.

Ustilago holubii Syd. 1935.

Ustilago crus-galli Tracy \& Earle var. minor Zundel 1953 (nom. inval.).

Sphacelotheca almorae A. Krishna \& R.A. Singh 1980. 
Sori in scattered flowers in the spike (usually affecting all floral parts, including ovaries), and on vegetative parts (leaves, stems) as bullate bodies from a few $\mathrm{mm}$ in diam. to $10 \mathrm{~cm}$ long swellings (especially on the stems), covered by a hispid peridium composed of an inner, fungal layer and an outer layer of host origin, which ruptures irregularly to expose the first agglutinated, later pulverulent, dark brown spore mass. Spores globose, subglobose to ovoid, 6-11 $\times 7-12 \mu \mathrm{m}$, pale olive-brown, sparsely to moderately densely verrucose-echinulate, spore profile wavy to sparsely serrulate, in SEM sparsely to moderately densely, minutely verruculose between the spines. Spore germination of Ustilago-type; basidium often two-celled or a short, aseptate basidium, occasionally with a lateral branch, successively produces ovoid to ellipsoidal basidiospores apically (Fig. 78).

On Poaceae: Echinochloa crus-galli (L.) P. Beauv., Golestan Prov., Bandargaz city; Khuzestan Prov., Ramin city; Kohgiluyeh va -Buyerahmad Prov., Dogonbadan city, IRAN 4401 F.

96. Ustilago tritici (Pers. : Pers.) Rostr., Overs. Kongel. Danske Vidensk. Selsk. Forh. Medlemmers Arbeider, 1890: 15, 1890b (March).

Uredo segetum Pers. var. tritici Pers. 1797. - Uredo segetum Pers. var. Uredo tritici Pers. : Pers. 1801. - Uredo carbo DC. var. tritici (Pers. : Pers.) DC. 1815. - Erysibe vera Wallr. var. tritici (Pers. : Pers.) Wallr. 1833. - Ustilago segetum ("Bulliard") Roussel var. tritici (Pers. : Pers.) Brunaud 1878. - Ustilago segetum (Pers.) Roussel var. tritici (Pers. : Pers.) Rostr. \& C.N. Jensen, in Jensen 1888. - Ustilago tritici (Pers. : Pers.) C.N. Jensen, in Kellerm. \& Swingle 1890 (June; later homonym).

Ustilago passerinii A.A. Fisch. Waldh. 1877b.

Ustilago ehrenbergiana A.A. Fisch. Waldh. 1879a, b.

Ustilago hordei Bref. 1888 (nom. ambig.).

Ustilago schumanniana Henn. 1893b.

Ustilago vavilovii Jacz. 1925.

Ustilago ugamica Golovin 1952.

Ustilago tritici (Pers. : Pers.) Rostr. [forma] folicola Henn. 1894.

Sori in spikelets, dark olive-brown, dusty ("loose smut"), usually destroying all floral parts leaving behind only the naked rachis, but in rye usually only parts of the lower spikelets are destroyed; occasionally also on leaves, sheaths and culms as striae. Spores globose to subglobose or ovoid, 5-7 $\times 6-9 \mu \mathrm{m}$, olive-brown, paler on one side, minutely echinulate. Spore germination results in septate, 4-celled basidia. On artificial media and in nature fusion between compatible basidial cells gives rise to dikaryotic hyphae which infect the host via ovary at flowering, resulting in a systemic disease (Fig. 79).

On Poaceae: Aegilops kotschyi Boiss., West Azarbaijan, Orumiyeh lake, Kabudan Island, IRAN 4348 F; Fars Prov., Bamu National Park, IRAN 13289 \& 13290 F.

On Aegilops. triaristata Willd., Ghazvin Prov., $50 \mathrm{~km}$ NW of Ghazvin city, IRAN 7280 F.

On Aegilops triuncialis L., Tehran Prov., Evin village, IRAN 6839 \& 8545 F; Ardebil Prov., Moghan city, between Ultan and Gharehaghaj villages, IRAN 8769 F; Fars Prov., Darab city, Layzangan village, IRAN 11537 F; Lorestan Prov., between Khoramabad and Alashtar cities, IRAN 8768 F; Ghazvin Prov., Chenarkhani village, IRAN 10425 F.

On Triticum aestivum L. (T. vulgare Vill.). Common in Iran.

97. Ustilago turcomanica Tranzschel ex Vánky, Mycotaxon 31: 404, 1988a.

Ustilago turcomanica Tranzschel, in Tranzschel \& Serebrianikow 1912 (nom. nud.).

Sori in spikelets, in ovaries and at the base of paleae and glumes, swollen, covered by a delicate membrane which ruptures exposing the dark brown, semi-powdery spore mass. Infection systemic; all flowers in a spike and usually all spikes of a plant affected. Spores globose, subglobose, ellipsoidal to somewhat irregular, 9-11 ×9.5-14(-15) $\mu \mathrm{m}$, medium olive-brown with slightly paler polar areas; wall $1-1.5 \mu \mathrm{m}$ thick, rarely almost smooth, usually densely and finely verruculose. Spore germination results in 2-3-septate basidia with 2-4 basal branches, in water giving rise to hyphae, in nutrient media developing lateral and terminal basidiospores (Fig. 80). 
On Poaceae: Eremopyrum bonaepartis (Spreng.) Nevski, Ghazvin Prov., $11 \mathrm{~km}$ W of Takestan, on the road of Takestan towards Ahar.

On Eremopyrum distans (K. Koch) Nevski, Semnan Prov., $20 \mathrm{~km}$ NE of Semnan city, IRAN $7252 \mathrm{~F}$.

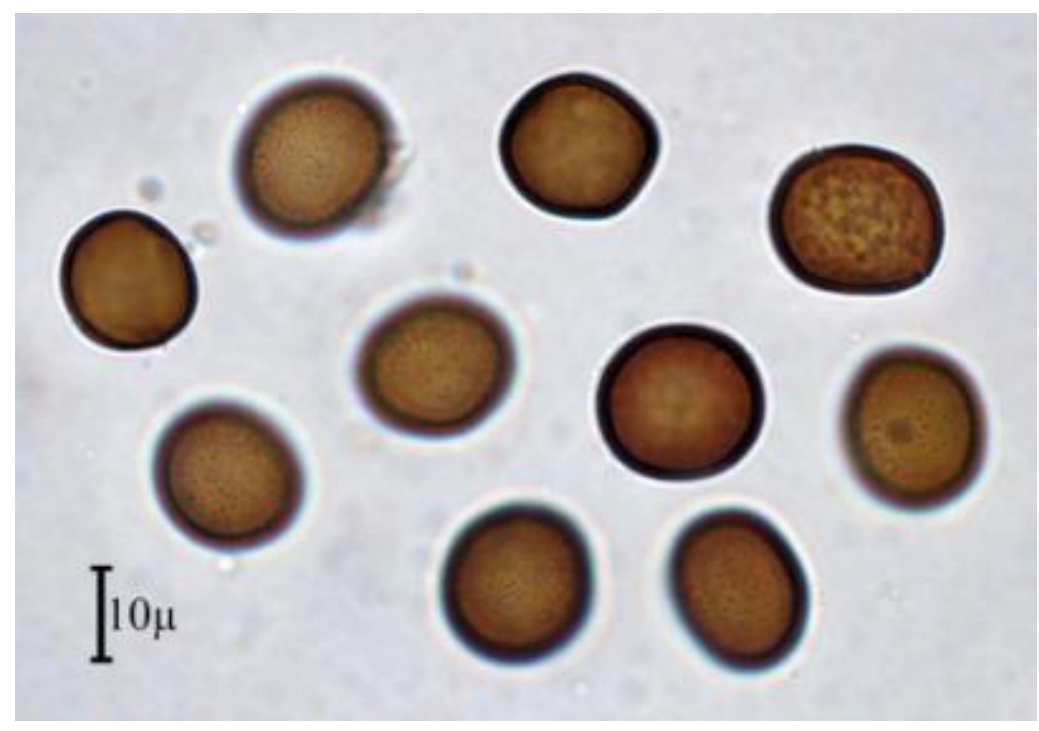

Fig.65 - Ustilago aeluropodis on Aeluropus sp. (11342)

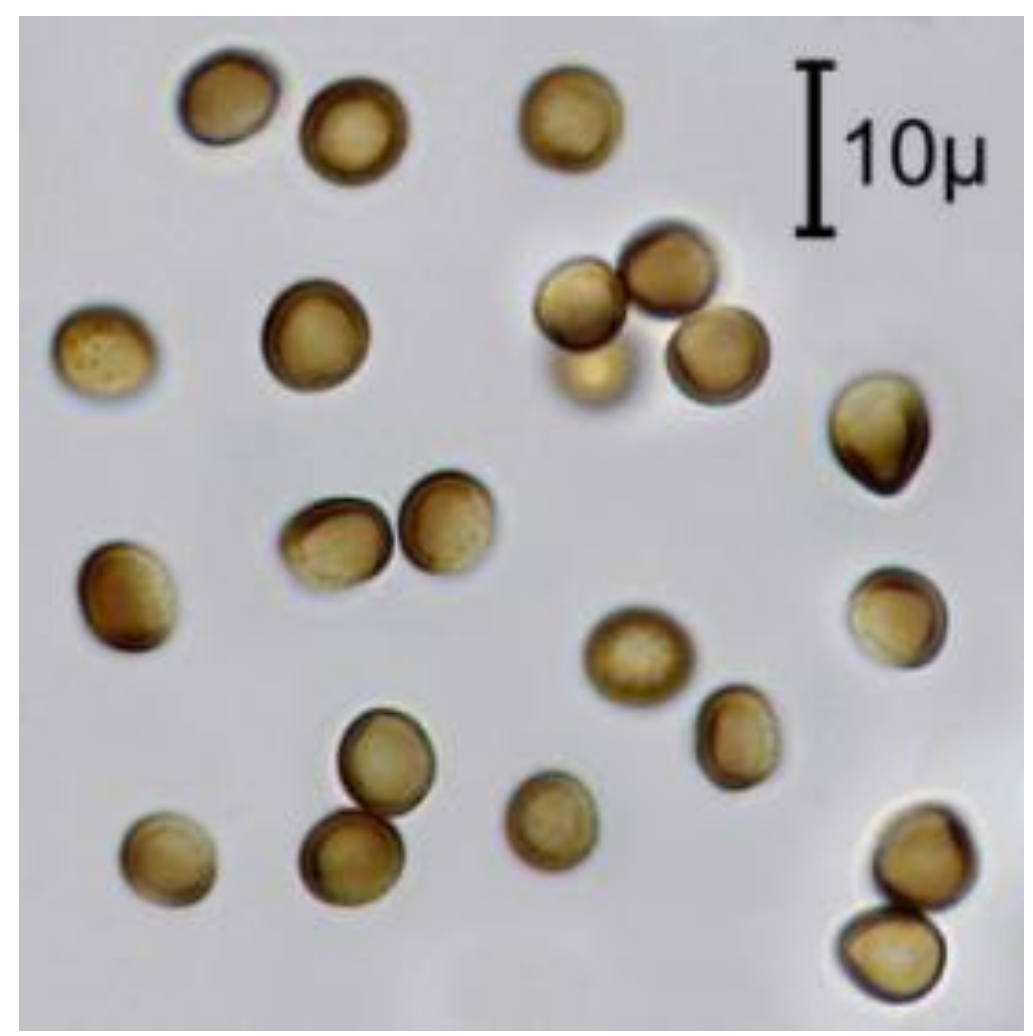

Fig.66 - Ustilago avenae on Avena ludoviciana (13873) 


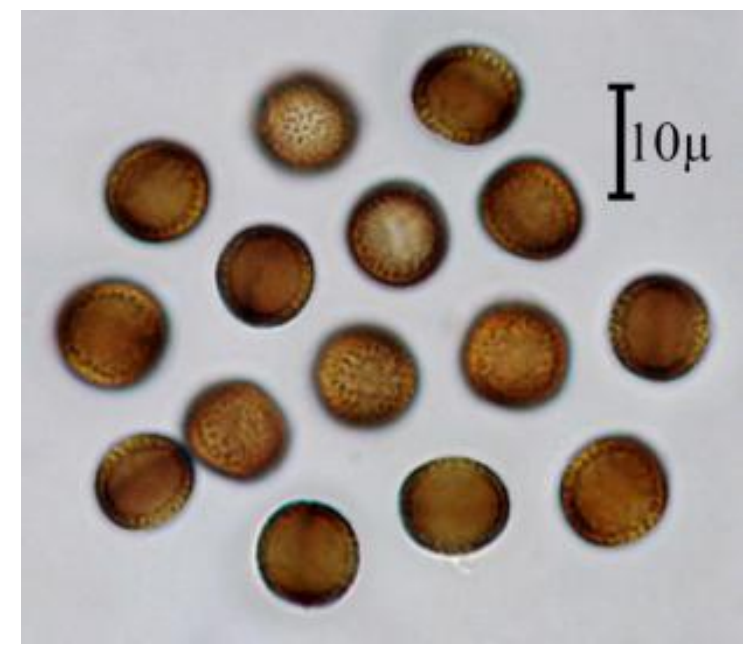

Fig.67 - Ustilago bromivora on Brachypodium distachyon (14785)

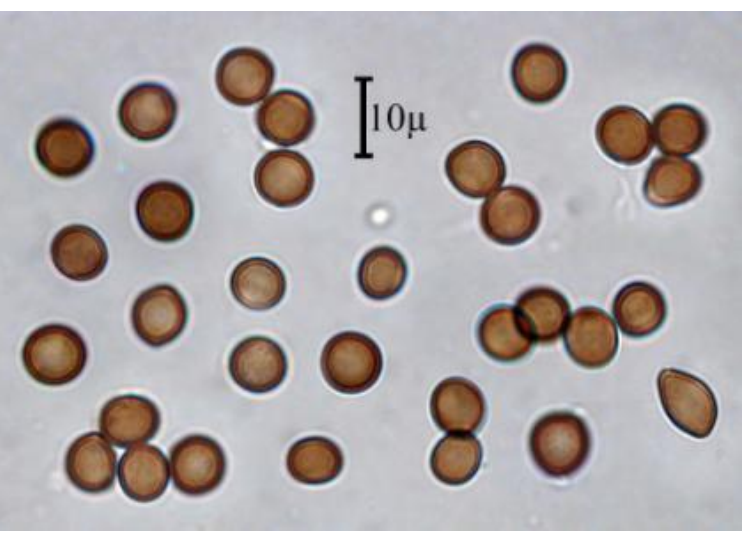

Fig.68 - Ustilago cynodontis on Cynodon dactylon (13284)

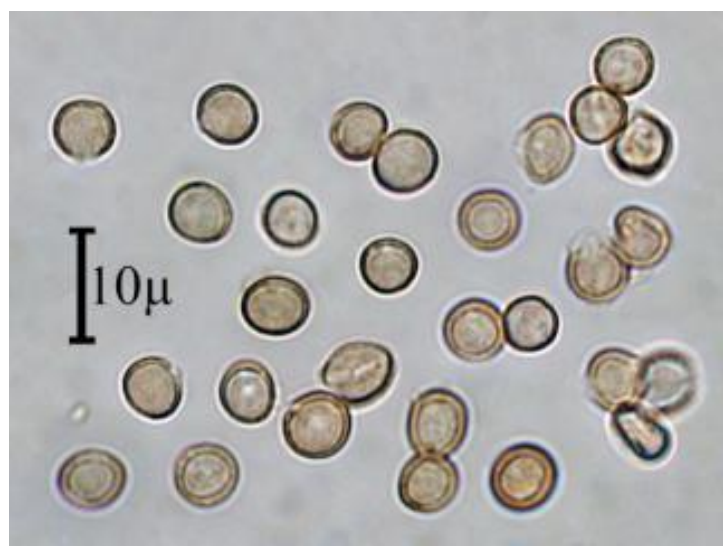

Fig.69 - Ustilago filiformis on Glyceria plicata (14216) 


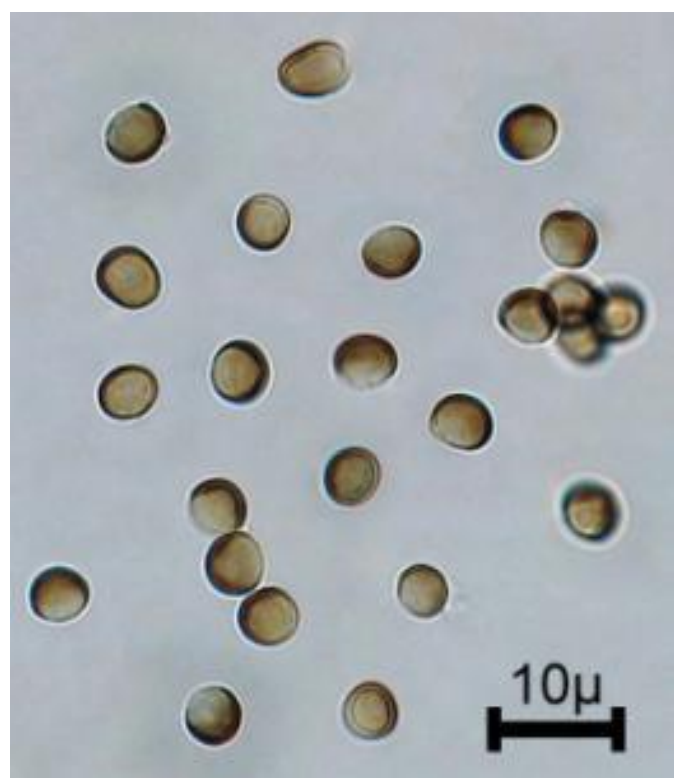

Fig.70 - Ustilago hordei on Hordeum vulgare (13870)

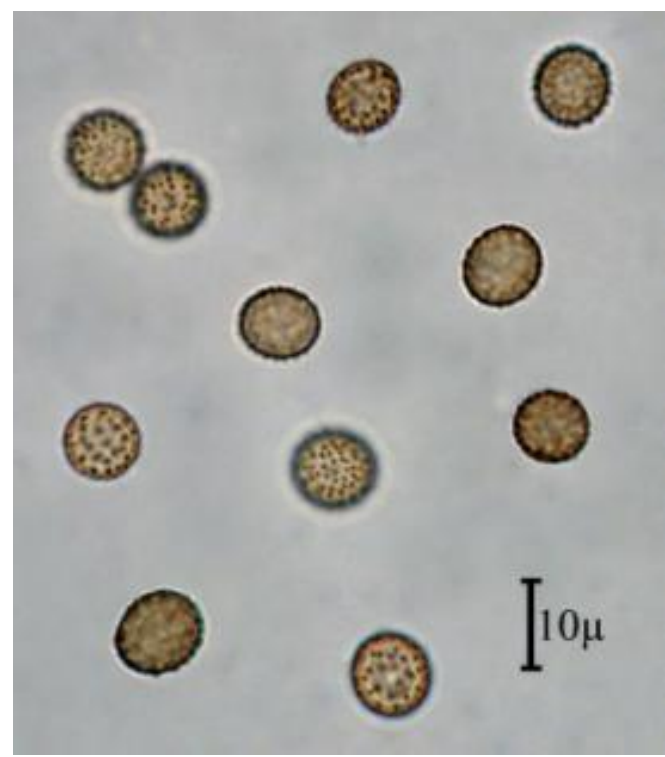

Fig.71 - Ustilago maydis on Zea mays (14571)

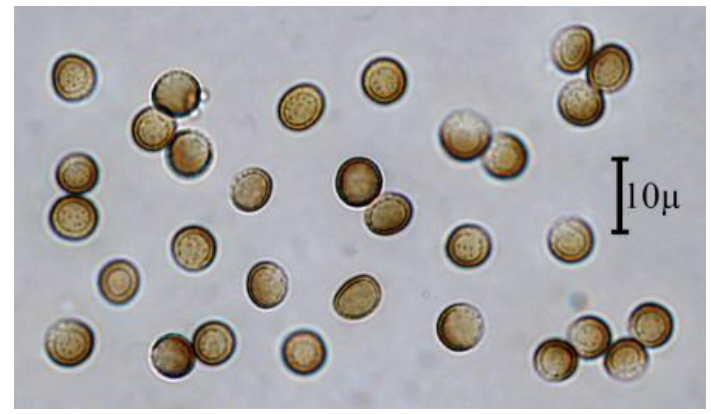

Fig.72 - Ustilago nuda on Hordeum vulgare (10528) 


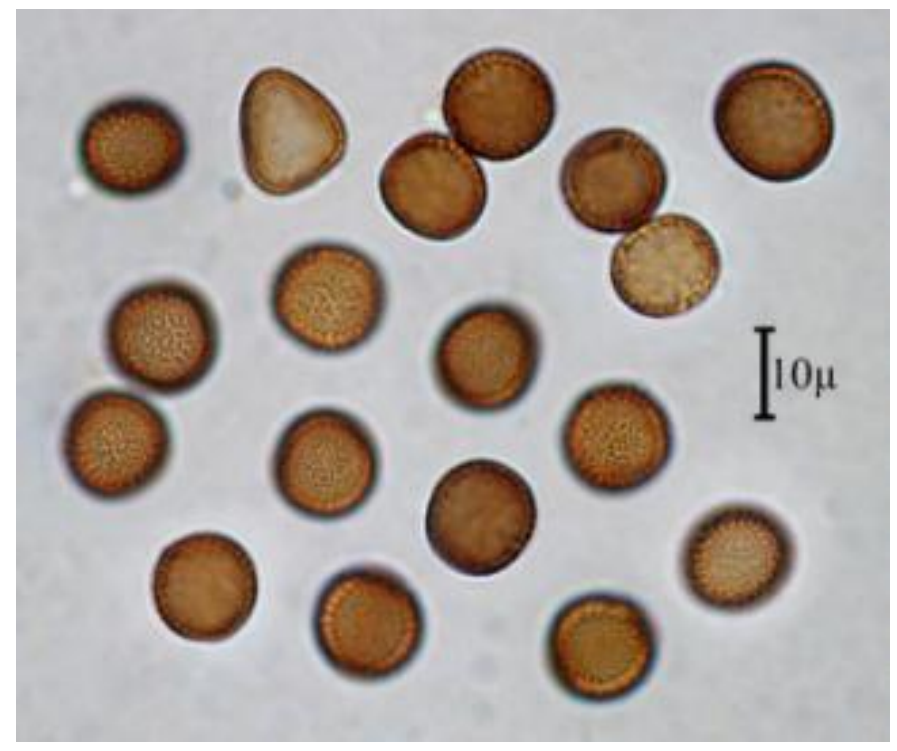

Fig.73 - Ustilago pamirica on Bromus gracillimus (7251)

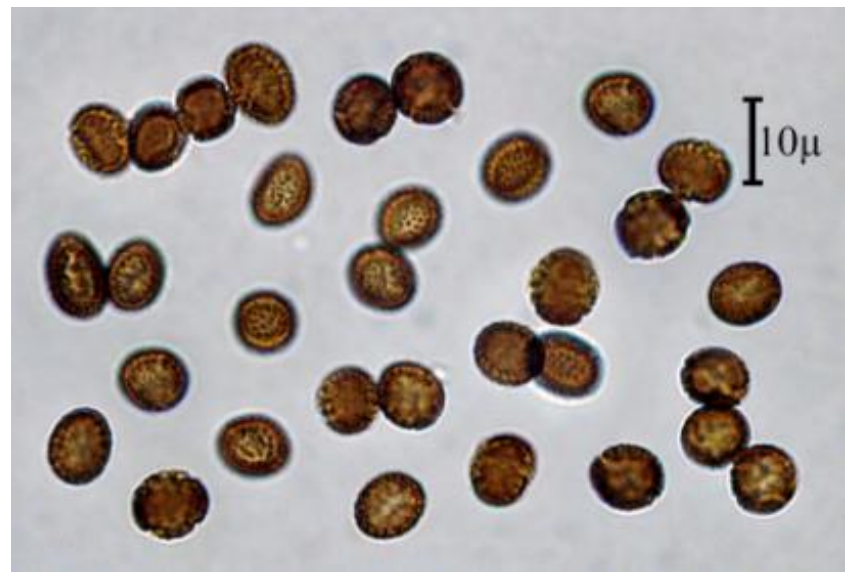

Fig.74 - Ustilago phrygica on Taeniatherum sp. (1764)

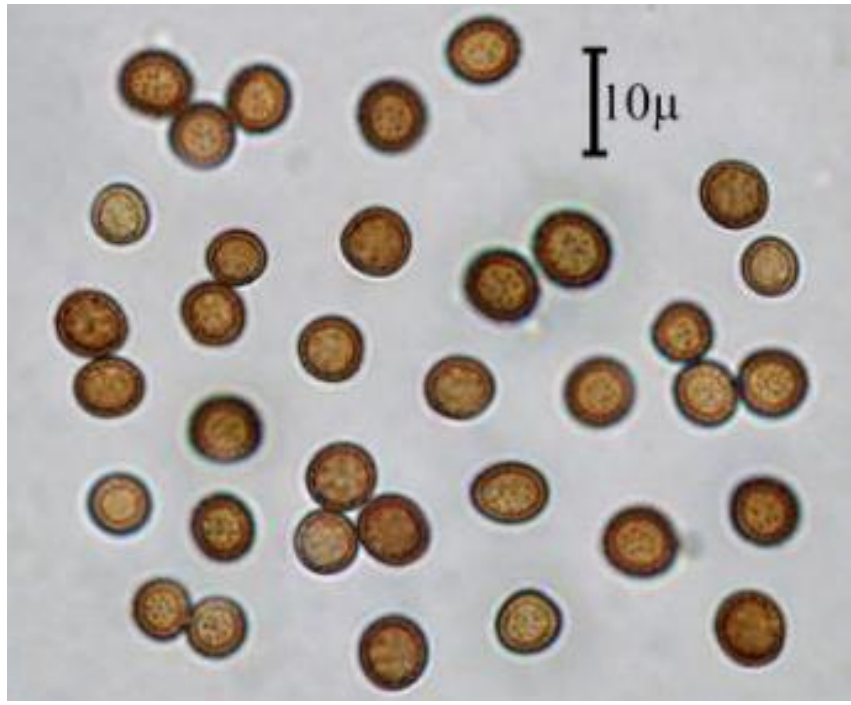

Fig.75 - Ustilago scitaminea on Saccharum officinarum (10712) 


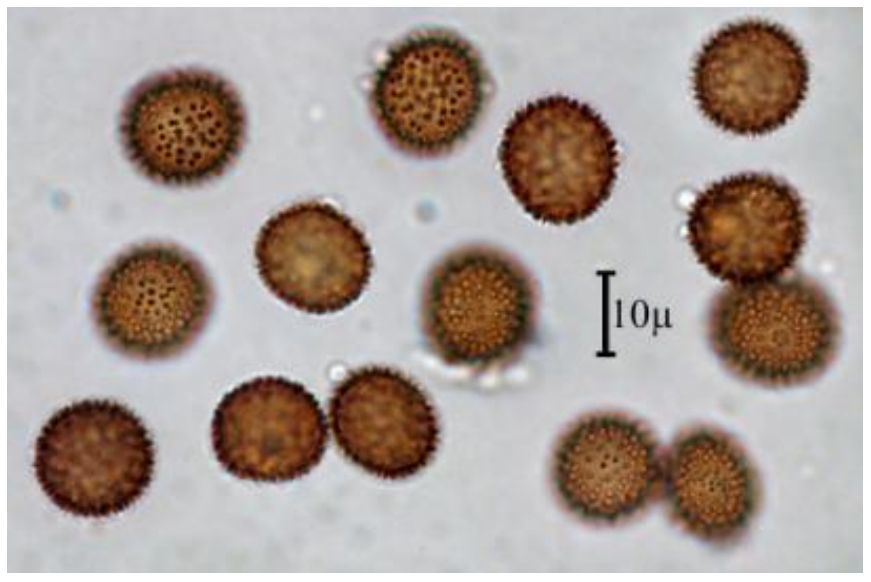

Fig.76 - Ustilago striiformis on unknown Poaceae (10499)

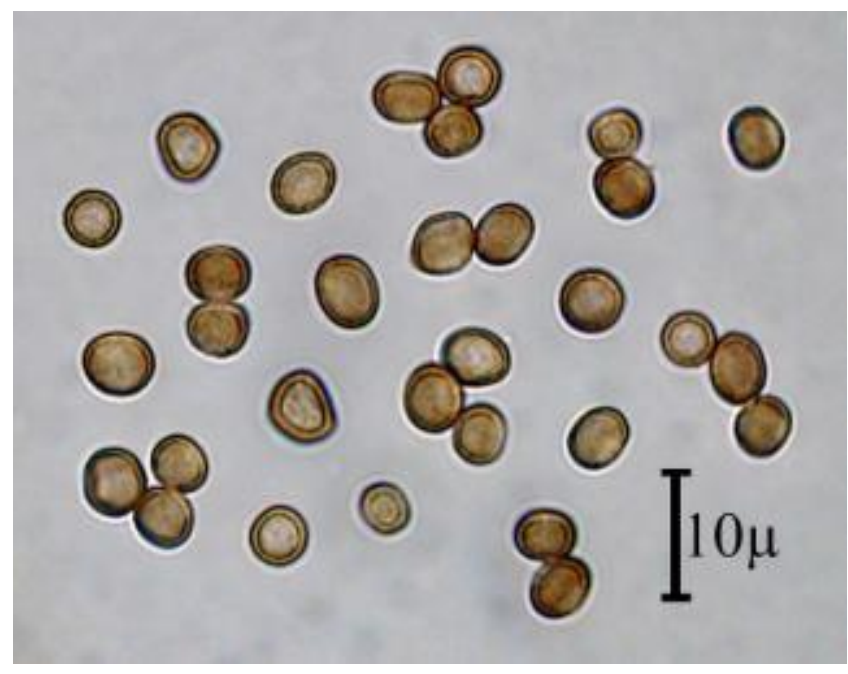

Fig.77 - Ustilago trebouxii on Bromus tomentellus (8088)

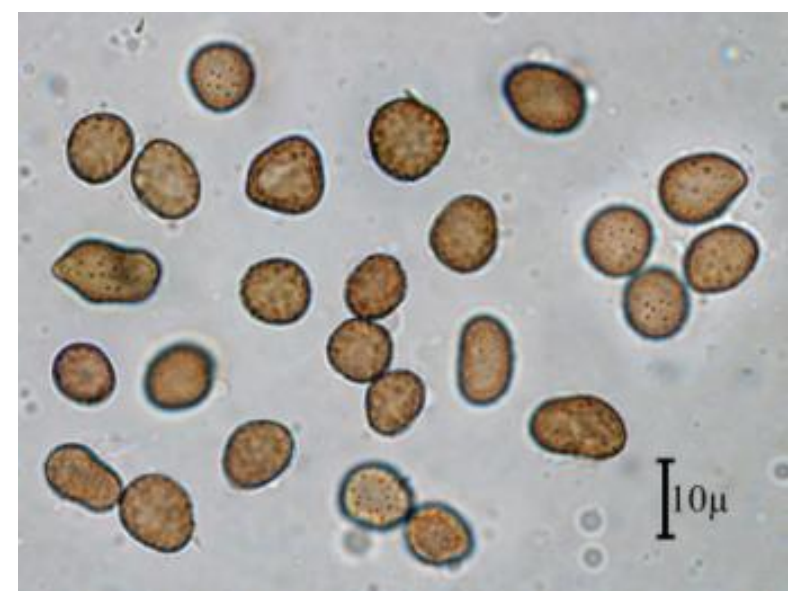

Fig.78 - Ustilago trichophora on Echinochloa crus-galli (4401) 


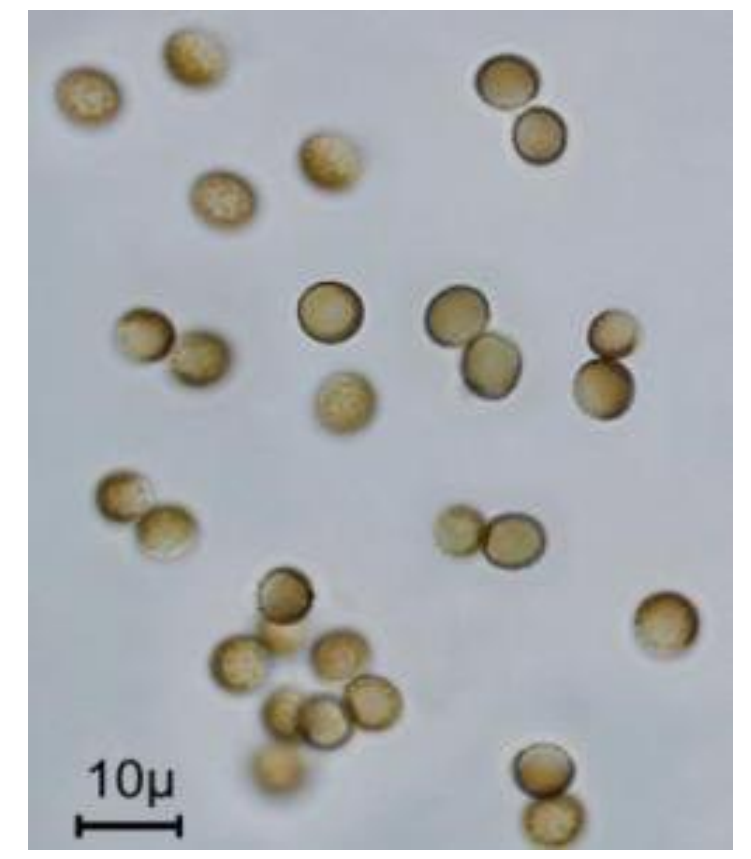

Fig.79 - Ustilago tritici on Triticum aestivum (14574)

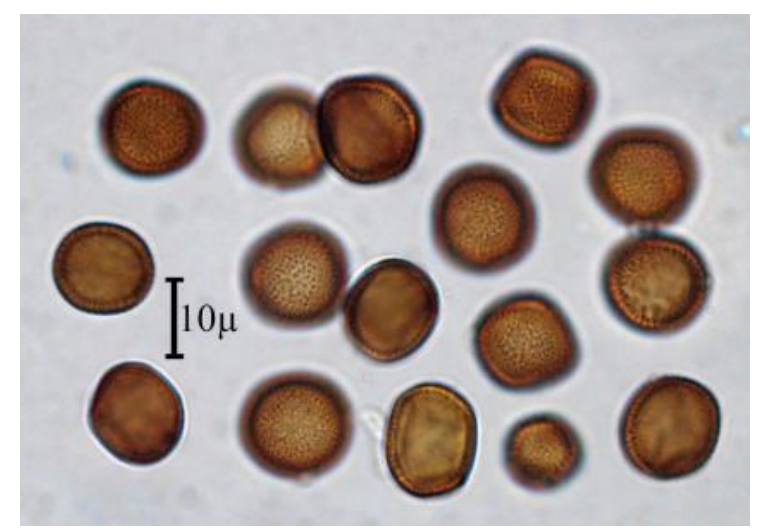

Fig.80 - Ustilago turcomanica on Eremopyrum distans (7252)

\section{Key to the Vankya species}

\section{Vankya Ershad}

1. Spores 15-23 $\mu \mathrm{m}$ long; wall 1.5-3 $\mu \mathrm{m}$ thick, with spines embedded in the exospore

- $\quad$ Spores 10.5-19(-24) $\mu \mathrm{m}$ long; wall $0.5-1.5 \mu \mathrm{m}$ thick, apparently smooth ........ o. ornithogali

98. Vankya heufleri (Fuckel) Ershad, Rostaniha 1: 68, 2000.

Ustilago heufleri Fuckel 1870.

Urocystis pompholygodes (Klotzsch) Rabenh. f. tulipae Rabenh. 1866 (nom. nud.) - Ustilago tulipae (Rabenh.) G. Winter 1881 (nom. illegit., superfl. pro U. heufleri).

Ustilago erythronii G.P. Clinton, in Peck 1873. - Ustilago ornithogali (J.C. Schmidt \& Kunze) J.G. Kühn f. erythronii (G.P. Clinton) De Toni, in Sacc. 1888.

Sori in leaves as elongate pustules, initially covered by a thin, whitish membrane which ruptures irregularly and flakes away to expose the blackish brown, dusty spore mass. Spores globose, subglobose to ovoid or slightly irregular, 13-19 × 15-23 $\mu \mathrm{m}$, olive-brown; spore wall two-layered, inner layer dark brown, c. $0.5 \mu \mathrm{m}$ thick, with sparsely, unevenly dispersed, $1-2 \mu \mathrm{m}$ high spines, the 
spines often arranged in irregular rows or groups, embedded in the outer, yellowish brown, 1-2.5 $\mu \mathrm{m}$ thick layer, sometimes reaching the spore surface but never exceeding it, in SEM almost smooth or with sparsely or moderately dense, low and wide warts. Sterile cells solitary, rarely in loosely connected pairs, indistinct, subglobose, ellipsoidal, to slightly irregular, collapsed in old specimens, 11-17(-20) $\mu \mathrm{m}$ long, yellowish to pale olivaceous brown, wall evenly or slightly unevenly $1.5-3(-4) \mu \mathrm{m}$ thick, one-layered, smooth. Spore germination results in two- to fourseptate basidia, $4-5 \times 70-80 \mu \mathrm{m}$, producing lateral, ovoid basidiospores which bud or germinate into hyphae (Fig. 81).

On Liliaceae: Tulipa biflora Pall., Esfahan Prov., Semirom city.

On Tulipa humilis Herb., Tehran Prov., Central Alborz, Tochal Mt., IRAN 8595 F.

On Tulipa montana Lindl., Tehran Prov., N. of Tehran city, Darabad Mt., IRAN 8510 F.

On Tulipa montana var. chrysantha (Boiss.) Wendelbo ex Rech. f., Tehran Prov., Alborz mountain, Palang-chal area, IRAN $8594 \mathrm{~F}$.

On Tulipa polychroma Stapf, Esfahan Prov., c. 135 km S. of Esfahan city, c. $20 \mathrm{~km} \mathrm{~N}$. of. Semirom city, IRAN 8509 F.

On Tulipa sp., West Azarbaijan Prov., Orumieh city, Ghushchi pass, IRAN 8761 F; Markazi Prov., Arak city, Lateh Dar Mt. IRAN 15001 F.

99. Vankya ornithogali (J.C. Schmidt \& Kunze) Ershad, Rostaniha 1: 66, 2000.

Uredo ornithogali J.C. Schmidt \& Kunze 1819. - Ustilago ornithogali (J.C. Schmidt \& Kunze)

Magnus 1875. - Ustilago ornithogali (J.C. Schmidt \& Kunze) J.G. Kühn, in Rabenh. 1875.

Caeoma ornithogali Schltdl. 1824.

Ustilago ornithogali J. Schröt., in Schneider 1869. (nom. nud.).

Ustilago umbrina J. Schröt. 1869.

Ustilago heterospora Niessl 1872.

Sori in leaves and bracts as 1-5(-10) $\mathrm{mm}$ long, fusiform swellings, initially covered by the epidermis, which ruptures longitudinally to expose the blackish brown, powdery spore mass. Infection systemic, appearing year after year on the same host plant. Spores variable in shape and size, irregular, subpolyhedral, elongate, acute, ovoid or subglobose, 9.5-15 × 10.5-19(-24) $\mu \mathrm{m}$, olive-brown, sometimes with a short pedicel; wall 0.5-1.5 $\mu \mathrm{m}$ thick, apparently smooth but actually very finely and densely punctate, spore profile smooth. Sterile cells few, solitary or in groups of 2-4, subglobose, ellipsoidal, slightly irregular, rarely elongate, collapsed in old specimens, 11-20 $\mu \mathrm{m}$ long, usually of the same colour as the spores; wall 1.5-3(-4) $\mu \mathrm{m}$ thick, one-layered, smooth (Fig. $82)$.

On Hyacinthaceae (Liliaceae s. lat.): Gagea confusa Terracc., Tehran Prov., $10 \mathrm{~km}$ N. of Tehran city, Darabad Mt., IRAN 8521 F.

On Gagea dubia Terracc., Tehran Prov., Tehran city, Tochal Mt., IRAN 8597 F; Chaharmahal va Bakhtiari Prov., 19 km W of Shahr-e-Kord city, Sureshjan village, IRAN 8522 F; Tehran Prov., Tehran city, Evin area, campus of Iranian Research Institute of Plant Protection, IRAN $9564 \mathrm{~F}$.

On Gagea. fistulosa (Ramond ex DC.) Ker Gawl., Chaharmahal va Bakhtiari Prov., Chelgerd city, Kuhrang city, IRAN 8208 F.

On Gagea gageoides (Zucc.) Vved., Tehran Prov., Tehran city, Tochal Mt. IRAN 8596 F; Tehran Prov., 60 km E of Tehran city, Elburz Mt., "Emamzadeh-Hashem" shrine, IRAN 7281 F; Tehran Prov., N. of Tehran city, Darabad Mt., IRAN 7282 F; Chaharmahal va Bakhtiari Prov., Chelgerd city, Zard-kuh Mt., IRAN 8085 F.

On Gagea sp., Chaharmahal va Bakhtiari Prov., Shahre Kord city, Sureshjan city, IRAN 8328 F; Esfahan Prov., 140 km NW of Esfahan city, Golestan Kuh Mt., IRAN 7284 F; Tehran Prov., N. of Tehran city, Darabad Mt., IRAN 7285 F. 


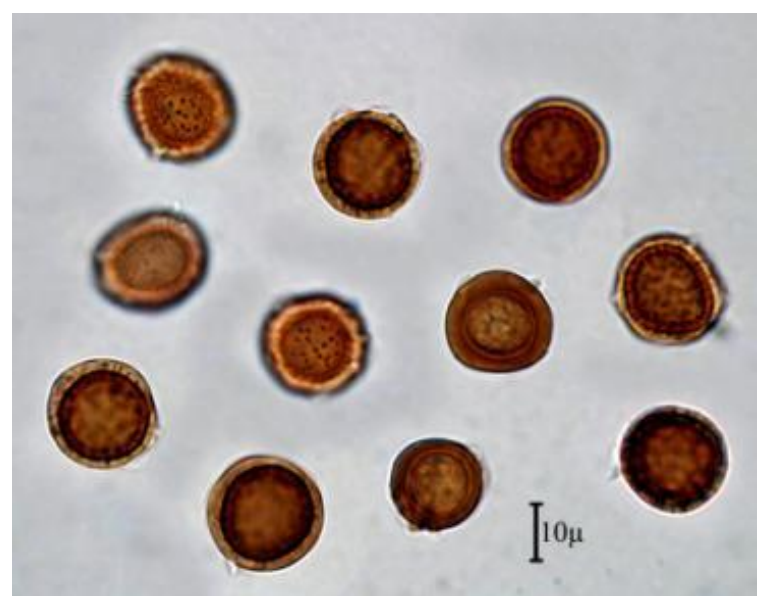

Fig.81 - Vankya heufleri on Tulipa polychroma (8509)

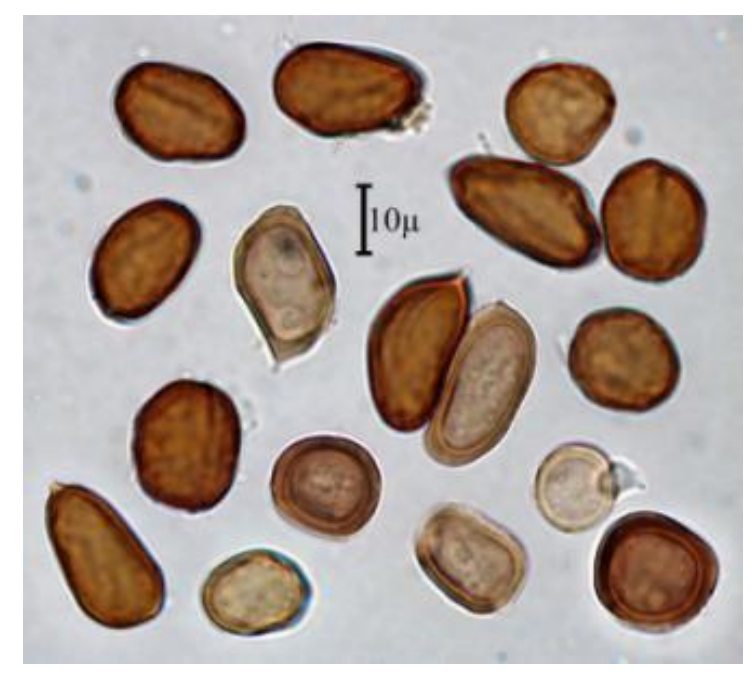

Fig.82 - Vankya ornithogali on Gagea confusa (8521)

\section{Host plant - smut fungus index}

Aegilops kotschyi - Ustilago tritici

Aegilops trinucialis - Tilletia contraversa; $T$. caries; Ustilago avenae; $U$. tritici

Aegilops. triaristata - Ustilago tritici

Aeluropus littoralis - Ustilago aeluropodis

Agropyron intermedium - Urocystis agropyri

Allium akaka-Urocystis magica

Allium cepa-Urocystis magica

Arrhenatherum kotschyi-Urocystis behboudii

Avena barbata-Ustilago avenae

Avena fatua - Ustilago avenae

Avena ludoviciana-Ustilago avenae

Avena sativa - Ustilago avenae

Avena sp. - Ustilago avenae

Bellevalia glauca-Antherospora tourneuxii

Bellevalia saviczii-Antherospora tourneuxii

Brachypodium distachyon - Ustilago bromivora 
Bromus danthoniae - Ustilago bromivora

Bromus gracillimus - Ustilago pamirica

Bromus japonicus - Ustilago bromivora

Bromus madritensis - Ustilago bromivora

Bromus rubens - Ustilago bromivora

Bromus sp. - Ustilago bromivora

Bromus stenostachyus - Ustilago striiformis

Bromus sterilis - Ustilago bromivora

Bromus tectorum - Ustilago bromivora

Bromus tomentellus - Ustilago trebouxii

Carex diluta-Anthracoidea caricetorum

Carex flacca ssp. serrulata-Anthracoidea pratensis

Carex halleriana - Schizonella cocconii

Carex liparocarpos - Anthracoidea caryophylleae

Carex melanostachya-Anthracoidea melanostachyae

Carex michelii-Anthracoidea michelii

Carex riparia - Farysia thuemenii

Carex songorica - Anthracoidea songorica

Carex sp. - Anthracoidea caricis; A. eleocharidis; Urocystis fischeri

Carex stenophylla - Anthracoidea eleocharidis

Carex tomentosa-Anthracoidea tomentosae

Ceratocephalus falcaus - Urocystis ceratocephali

Colchicum procurrens - Urocystis colchici

Colchicum sp. - Urocystis colchici

Critesion bulbosum - Tilletia contraversa

Critesion marinum - Tilletia hordei

Critesion murinum - Tilletia hordei

Critesion murinum subsp. leporinum - Tilletia hordei

Critesion violaceum - Urocystis tianschanica

Cymbopogon olivieri - Sporisorium lanigeri

Cynodon dactylon - Ustilago cynodontis

Cynodon sp. - Ustilago cynodontis

Dactylis glomerata - Jamesdicksonia dactylidis; Ustilago striiformis

Dianthus caryophyllus - Microbotryum dianthorum

Dianthus orientalis - Microbotryum dianthorum

Dianthus tabrisianus - Microbotryum dianthorum

Dichanthium annulatum - Sporisorium andropogonis; Sp. foveolati

Dichanthium foveolatum - Sporisorium foveolati

Dichanthium ischaemum - Sporisorium andropogonis

Echinochloa crus-galli-Moesziomyces bullatus; Ustilago trichophora

Elymus hispidus - Tranzscheliella hypodytes; Tilletia contraversa

Elymus hispidus subsp. barbulatus - Tilletia contraversa; Tranzscheliella hypodytes

Elymus repens - Tranzscheliella hypodytes

Elymus sp. - Tranzscheliella hypodytes

Eremopyrum bonaepartis - Ustilago turcomanica

Eremopyrum distans - Ustilago turcomanica

Gagea confusa - Vankya ornithogali

Gagea dubia - Vankya ornithogali

Gagea fistulosa - Vankya ornithogali

Gagea gageoides - Vankya ornithogali 
Gagea sp. - Vankya ornithogali

Geranium tuberosum - Entyloma atlanticum

Gladiolus segetum - Urocystis gladiolicola

Glyceria plicata - Ustilago filiformis

Hordeum bulbosum - Jamesdicksonia dactylidis

Hordeum glaucum - Ustilago bromivora

Hordeum leporinum - Ustilago hordei

Hordeum sp. - Ustilago hordei

Hordeum spontaneum - Jamesdicksonia dactylidis

Hordeum vulgare - Tilletia contraversa; Ustilago avenae; U. hordei; U. nuda

Hydrocharis morsus-ranae - Tracya hydrocharidis

Hyparrhenia hirta - Sporisorium vanderystii

Imperata cylindrica - Sporisorium schweinfurthianum

Ixiolirion pallasii - Urocystis ixiolirii

Ixiolirion sp. - Urocystis ixiolirii

Lasiurus sindicus - Sporisorium desertorum

Linaria sp. - Entyloma linariae

Loliolum subulatum - Tilletia lolioli

Lolium loliaceum - Urocystis bolivarii

Lolium rigidum - Tilletia lolii; Urocystis bolivarii

Lolium sp. - Urocystis bolivarii

Muscari sp. - Antherospora vaillantii; Urocystis muscaridis

Myosotis sylvatica - Entyloma fergussonii

Ornithogalum kurdicum - Urocystis ornithogali

Orobanche ramosa-Urocystis orobanches

Panicum miliaceum - Sporisorium destruens

Pedicularis sibthorpii-Urocystis pedicularis

Pennisetum orientale - Sporisorium penniseti

Phalaris sp. - Urocystis phalaridis

Physalis alkekengi-Entyloma australe

Poa annua - Ustilago striiformis

Poa sp. - Ustilago striiformis

Poa trivialis - Ustilago striiformis

Primula veris subsp. macrocalyx - Urocystis primulae

Psathyrostachys fragilis - Tilletia hordei

Ranunculus ficaria - Entyloma majewskii; Urocystis ficariae

Ranunculus muricatus - Entyloma microsporum; Urocystis ranunculi

Ranunculus sceleratus - Entyloma ranunculi-repentis

Rhagadiolus stellatus - Entyloma rhagadioli

Rostraria cristata - Tilletia rostrariae

Saccharum officinarum - Ustilago scitaminea

Schismus arabicus - Sporisorium aegyptiacum

Scorzonera caliculata-Microbotryum scorzonerae

Setaria glauca-Macalpinomyces neglectus

Setaria italica - Ustilago crameri

Setaria viridis - Ustilago crameri

Sorghum bicolor - Sporisorium cruentum; Sp. ehrenbergii; S. reilianum; Sp. sorghi

Sorghum cernuum - Sporisorium sorghi

Sorghum halepense - Sporisorium cruentum; Sp. reilianum; Sp. sorghi

Sorghum sp. - Sporisorium cruentum; Sp. sorghi 
Sorghum vulgare - Sporisorium reilianum

Spodiopogon pogonanthus - Sporisorium persicum

Stipa capensis - Urocystis corsica

Stipa hohenackeriana-Tranzscheliella williamsii

Stipa pulcherrima - Tranzscheliella williamsii

Stipa sp. - Tranzscheliella iranica

Stipagrostis plumosa-Sporisorium aristidae-lanuginosae

Taeniatherum asperum - Tilletia bornmuelleri

Taeniatherum caput-medusae - Ustilago phrygica

Taeniatherum crinitum - Ustilago phrygica

Taeniatherum sp. - Ustilago phrygica

Thalictrum foetidum - Urocystis sorosporioides

Thalictrum sultanabadense - Urocystis sorosporioides

Tragopogon graminifolius - Entyloma tragopogonis; Microbotryum tragopogonis-pratensis

Tricholaena teneriffae - Sporisorium tricholaenae

Triticum aestivum - Tilletia caries; T. contraversa; T. indica; T. laevis; Urocystis tritici; Ustilago tritici

Triticum sp. - Tilletia caries; Urocystis tritici

Tulipa biflora - Vankya heufleri

Tulipa humilis - Vankya heufleri

Tulipa montana - Vankya heufleri

Tulipa montana var. chrysantha - Vankya heufleri

Tulipa polychroma - Vankya heufleri

Tulipa sp. - Vankya heufleri

Viola occulta - Urocystis kmetiana

Viola sp. - Urocystis violae

Viola tricolor - Urocystis kmetiana

Zea mays - Sporisorium reilianum; Ustilago maydis

\section{Acknowledgment}

The help of Ms. S. Samadi, former MSc student, Biology Department, Tehran University with the first draft of the distribution of the Iranian smut fungi, as well as help with the microphotographs is gratefully acknowledged.

\section{References}

Ahmad S. 1956 - Ustilaginales of West Pakistan. Mycol. Pap. 64, 1-17 pp.

Ahmad S. 1969 - Fungi of West Pakistan. Supplement I. Biological Society of Pakistan. Monograph No. 5. Lahore, West Pakistan.

Ainsworth G.C. 1949 - The Gladiolus smut. Trans. Brit. Mycol. Soc. 32, 255-257.

Albertini JB, Schweinitz LDv 1805. - Conspectus fungorum in Lusatiae superioris agro Niskiensi crescentium. Lipsiae, XXIX + 376 pp.

Allescher A, Hennings P. 1897 - Pilze aus dem Umanakdistrict, etc. Bibliotheca Botan. 12, 40-54 (n.v.).

Appel O, Gassner G. 1907. Der Brand des Raygrasses. Mitt. Kaiserl. Biol. Anst. Land.Forstwirtsch. 4, 12-14.

Auerswald B. 1855. In Rabenhorst L., Klotzschii Herbarium vivum mycologicum, etc. Cent. XX. Bot. Zeitung (Berlin) 13, 302.

Baccarini P. 1906. Funghi dell' Eritrea. Ann. Bot. (Rome) 4, 269-277 + Pl. X.

Bag MK, Agarwal DK. 2001. Taxonomic studies on smut fungi from North-Eastern India. Indian Phytopathol. 54, 219-225. 
Bary A. de 1874. Protomyces microsporus und seine Verwandten. Bot. Zeitung (Berlin) 32, 97-108.

Bauer R, Oberwinkler F, Vánky K. 1997. Ultrastructural markers and systematics in smut fungi and allied taxa. Canad. J. Bot. 75, 1273-1314.

Bauer R, Begerow D, Nagler A, Oberwinkler F. 2001. The Georgefischeriales: a phylogenetic hypothesis. Mycol. Res. 105, 416-424.

Bauer R, Lutz M, Begerow D, Piatek M, Vánky K, Bacigálová K, Oberwinkler F. 2008. Anther smut fungi on monocots. Mycol. Res. 112, 1297-1306.

Becerescu D. 1970. Ustilago haynaldiae sp. nov. Rev. Roumanie Biol., Ser. Bot., 15, 233-243.

Beckmann J. 1768. Des Herrn Tillet Beobachtung einer Krankheit des türkischen Weizens oder des Mais. Hannover. Mag. 6, 1329-1339.

Beeli M. 1922. Notes mycologiques. Bull. Jard. Bot. État 8, 1-22.

Berkeley MJ. 1874. Notices of North American fungi. Grevillea 3, 49-64.

Berkeley MJ, Broome CE. 1850. Notices of British fungi. Ann. Mag. Nat. Hist., Ser. 2, 5, 455-466.

Berkeley MJ, Broome CE. 1875. Notices of British fungi. Ann. Mag. Nat. Hist., Ser. 4, 15, $28-41$.

Berkeley MJ, Broome CE. 1880. List of fungi from Brisbane, Queensland; with descriptions of new species. Trans. Linn. Soc. London, Bot., Ser. 2, 1, 399-407.

Biedenkopf H. 1894. Ustilago medians, ein neuer Brand auf Gerste. Z. Pflanzenkrankh. 4, 321-322.

Bizzozero G. 1885. Flora Veneta Crittogamica. Parte I. I funghi. Padova. 572 pp.

Bjerkander C. 1775. Anmärkningar vid kol- eller sot-ax i hvetet. Kongl. Vetensk. Acad. Handl. 36, 317-329.

Boidol M, Poelt J. 1963. Zur Kenntnis der Blütenbrände von Cyperaceen in Südbayern. Ber. Bayer. Bot. Ges. 36, 13-24.

Brefeld O. 1888. Neue Untersuchungen über die Brandpilze und die Brandkrankheiten. II. Nachr. Klub Landwirthe Berlin No. 220-222, 1577-1584, 1588-1594, 1597-1602.

Brefeld O. 1895. Untersuchungen aus dem Gesammtgebiete der Mykologie. XII. Hemibasidii. Brandpilze III. Münster i. W., Commissions-Verlag v. H. Schöningh. IV + 99-236 pp. + Pls. VI-XII.

Brefeld O. 1912. Untersuchungen aus dem Gesammtgebiete der Mykologie. XV. Die Brandpilze und die Brandkrankheiten. V. Münster i. W., Commissions-Verlag v. H. Schöningh. V + 1151 pp. + Pls. I-VII.

Brockmüller H. 1863. 4. Beiträge zur Kryptogamen-Flora Mecklenburgs. Arch. Vereins Freunde Naturgesch. Meklenburg 17, 162-256.

Brunaud P. 1878. Liste des plantes phanérogames et cryptogames croissant spontanément à Saintes (Charente-Inférieure) et dans les environs. Actes Soc. Linn. Bordeaux, Vol. 32, Ser. 4, 2, 116-170.

Bubák F. 1909. Ein neue Tilletia-Art. Z. Landw. Versuchswesen Oesterr. 1909, 545-549.

Bubák F. 1912. Houby České. Díl II. Sněti (Hemibasidii). Arch. Př́ŕr. Výzk. Čech. 15(3), 1-84.

Bubák F. 1914. Fungi. Wissenschaftliche Ergebnisse der Expedition nach Mesopotamien, 1910. Ann. Naturhist. Hofmus. 28, 189-218 + 2 Pls.

Bubák F. 1922. Une nouvelle espèce du genre Urocystis. Bol. Soc. Esp. Hist. Nat. 22, 205-207.

Buhse F. 1860. Aufzählung der einer Reise durch Transkaukasien und Persien gesammelten Pflanzen (in Gemeinschaft mit Edmound Boissier). Moskau, typ. Gautier. 4. LXVII, LV, 248 pp., 10 tab., 1 mappa geogr.

Bornmüller J. 1908. Beiträge zur flora der Elbursgebirge Nord-Persiens, Fungi. Bull. Herb. Boissier, sér. 2, 8, 917-922.

Bulliard JBF. 1791. Histoire des champignons de la France, etc. I. Paris. (n.v.).

Busse W. 1905. Untersuchungen über die Krankheiten der Sorghum-Hirse. Ein Beitrag zur Pathologie und Biologie tropischer Kulturgewächse. Arbeiten Biol. Abt. Land- Forstw. Kaiserl. Gesundheitsamt 4, 319-426 + P1. 5.

Candolle de AP. 1805. Flore française, etc., Ed. 3, Vol. 2. Paris, Agasse.

Candolle de AP. 1815. Flore française. Ed. 3, Vol. 6. Paris, Desray. 660 pp. 
Ciferri R. 1924. Prima contribuzione allo studio degli Ustilaginales (No. 1-22). Bull. Soc. Bot. Ital. 1924, 46-59.

Ciferri R. 1925. Terza contribuzione allo studio degli Ustilaginales. Alcuni micromiceti della flora Spagnola e Svizzera. Atti Ist. Bot. Univ. Pavia, Ser. 3, 2, 7-14.

Ciferri R. 1928. Quarta contribuzione allo studio degli Ustilaginales. Ann. Mycol. 26, 1-68 + Pl.

Ciferri R. 1931. Quinta contribuzione allo studio degli Ustilaginales. Ann. Mycol. 29, 1-74.

Ciferri R. 1933. Ustilaginales esotici nuovi o rari. I. Nuovo Giorn. Bot. Ital., N. S. 40, 252-268.

Ciferri R. 1938. Ustilaginales. In Flora Italica Cryptogama, Pars I. Fungi, Fasc. 17, pp. 1-443.

Ciferri R. 1959. Revision of the Ustilaginaceous smut fungi on Polygonaceae. In Omagiu lui Traian Săvulescu. Edit. Acad. R.P.Romane, Bucureşti, pp. 163-173.

Ciferri R. 1963. Revisio Ustilaginearum. (Pars I. Tilletiaceae). Quaderno 27, 1-431.

Clinton GP. 1897. Broom-corn smut. Univ. Illinois Agric. Exp. Sta. Bull. 47, 373-412.

Clinton GP. 1900. The smuts of Illinois agricultural plants. Univ. Illinois Agric. Exp. Sta. Bull. 57, 289-360.

Clinton GP. 1902. North American Ustilagineae. J. Mycol. 8, 128-156.

Cooke MC. 1876. Some Indian fungi. Grevillea 4, 114-118 + Pl. 63.

Cooke MC. 1889. Some exotic fungi. Grevillea 18, 34-35.

Corda ACJ. 1842. Icones fungorum hucusque cognitorum, Vol. 5. Pragae.

Cornu M. 1883. Sur quelques Ustilaginées nouvelles ou peu connues. Ann. Sci. Nat. Bot., Sér. 6, 15, 269-296.

Crowell IH. 1942. Two new Canadian smuts. Canad. J. Res., Sect C., Bot. Sci. 20, 327-328.

Curzi M, Barbaini M. 1927. Fungi Aternenses, etc. Atti Ist. Bot. Univ. Pavia, Ser. 3, 3, 147-202.

Davis WH. 1928. Two physiological forms of Ustilago striaeformis (Westd.) Niessl. Phytopathology $18,149$.

Davis WH. 1935. Summary of investigations with Ustilago striaeformis parasiting some common grasses. Phytopathology 25, 810-817.

Deml G, Oberwinkler F. 1982. Studies in Heterobasidiomycetes, Part 24. On Ustilago violacea (Pers.) Rouss. from Saponaria officinalis L. Phytopathol. Z. 104, 345-356.

Denchev CM. 1995. A comparative taxonomical investigation of Ustilentyloma pleuropogonis and U. fluitans (Ustilaginales). Mycotaxon 55, 243-254.

Denchev CM, Denchev TT. 2011. Anthracoidea melanostachyae, sp. nov. (Anthracoideaceae). Mycol. Balcan. 8, 153-155.

Desmazières JBHJ. 1840. Notice sur quelques plantes cryptogames nouvellement découvertes en France, etc. Ann. Sci. Nat. Bot., Ser. 2, 13, 181-190.

Dietel P. 1893. Descriptions of new species of Uredineae and Ustilagineae, with remarks on some other species. I. Bot. Gaz. (Crawfordsville) 18, 253-256.

Dingley JM, Versluys W. 1977. Occurrence of Urocystis junci, Tranzscheliella williamsii (Griff.) comb. nov., and Ustilago hypodytes in New Zealand. New Zealand J. Bot. 15, 477-480.

Duby JE. 1830. Botanicon Gallicum, etc. Ed. 2, Pars 2. Paris. 545-1068 pp.

Durán R, Fischer GW. 1961. The genus Tilletia. Washington State University, 138 pp.

Durrieu G, Zambettakis C. 1973. Les Ustilago parasites des Caryophyllacées. Apports de la microscopie électronique. Bull. Soc. Mycol. France 89, 283-290.

Ellis JB, Everhart BM. 1895. New species of Ustilagineae and Uredineae. Bull. Torrey Bot. Club 22, 57-61.

Ellis JB, Everhart BM. 1897. New species of fungi from various localities. Bull. Torrey Bot. Club 24, 457-477.

Endlicher S. 1830. Flora Posoniensis. Posonii.

Ershad D. 1977. Fungi of Iran. Tehran. Dept. of Botany, Publication No. 10, 277 pp.

Ershad D. 1994. Contribution to the knowledge of smut fungi of Iran. Iran. J. Pl. Pathol. 30, 17-22.

Ershad D. 1998('1997'). New findings on smut fungi from Iran. Iran. J. Pl. Pathol. 33, 59-61 (in English) \& 174-176 (in Farsi). 
Ershad D. 2000. Vankya, a new genus of smut fungi. Rostaniha 1, 65-72 (in English) \& 151-161 (in Farsi, and Figs. 1-6).

Ershad D. 2001. Smut fungi reported from Iran. Rostaniha. Supplement 1, 1-254 (in Farsi).

Ershad D. 2003. Urocystis ornithogali, a smut fungus new to Iran. Rostaniha 4, 47-50.

Ershad D, Amini Rad M. 2003. Two Anthracoidea species new to Iran. Rostaniha 4, 151-154.

Ershad D, Amini Rad M. 2004. Anthracoidea michelii, a smut new to Iran. Rostaniha 5, 120-121.

Esfandiari E. 1946. Contribution à l'étude de la Mycoflore de l' Iran. Ministère de l'Agriculture, Department general de la protection des plantes, Tehran. 34 pp.

Esfandiari E. 1948. Les charbons des céréales en Iran. Entomol. Phytopathol. Appl. 6-7, 48-68.

Esfandiari E. 1951. Quatrieme liste de fungi de l'Iran. Entomol. Phytopathol. Appl. 12-13, 1-26.

Esfandiari E, Petrak F. 1950. Pilze aus Iran. Sydowia 4, 11-38.

Farlow WG. 1877. The onion smut. Rep. (Annual) Secretary Mass. State Board Agric. 24, 164-176.

Farlow WG. 1883. Notes on some Ustilagineae of the United States. Bot. Gaz. 8, 271-278.

Ferle F. 1912. Verzeichnis parasitischer Pilze, etc. Korrespondenzbl. Naturf.-Vereins Riga 55, 103106.

Fischer GW. 1938. Some new grass smut records from the Pacific Northwest. Mycologia 30, 385395.

Fischer GW. 1952. Some new species of Ustilaginales from North America. Res. Stud. State Coll. Wash. 20, 3-10.

Fischer GW. 1952. Tilletia brevifaciens sp. nov., causing dwarf bunt of wheat and certain grasses. Res. Stud. State Coll. Wash. 20, 11-14.

Fischer GW, Hirschhorn E. 1945. A critical study of some species of Ustilago causing stem smut on various grasses. Mycologia 37, 236-266.

Fischer von Waldheim AA. 1867. Sur la structure des spores des Ustilaginées. Bull. Soc. Imp. Naturalistes Moscou 40, 242-261.

Fischer von Waldheim AA. 1877a('1876'). Les Ustilaginées et leurs plantes nourricières. Ann. Sci. Nat. Bot., Sér. 6, 4, 190-276.

Fischer von Waldheim AA. 1877b. Aperçu systématique des Ustilaginées, leurs plantes nourricières et la localisation de leurs spores. Paris, Lahure, $51 \mathrm{pp}$.

Fischer von Waldheim AA. 1877c. Aperçu systématique des Ustilaginées, leurs plantes nourricières et la localisation de leurs spores. -Hedwigia 15, 100-108.

Fischer von Waldheim AA. 1878. Ustilago thümenii. Ein neuer südamerikanischer Brandpilz. Hedwigia 17, 40.

Fischer von Waldheim AA. 1879a. O nekotorych novych golovnevych. (On some new smuts). Vars. Univ. Izv. 1879(2), Neoffic. Otd. 2, 11-15, 1879.

Fischer von Waldheim AA. 1879b. Über die von G. Ehrenberg in Aegypten und Nubien gesammelten Brandpilze. Hedwigia 18, 100.

Fischer von Waldheim AA. 1880. Über zwei neue aussereuropäische Brandpilze. Verh. Bot. Vereins Prov. Brandenburg 22, 65.

Fries E. 1829-1832 ("1832"). Systema mycologicum, etc., Vol. 3, sectio 1, 1-260 (1829); sectio 2, 261-524 (1832). Gryphiswaldae.

Fuckel L. 1860. Enumeratio fungorum Nassoviae. Jahrb. Vereins Naturk. Herzogth. Nassau 15, 1126.

Fuckel L. 1870 ('1869 \& 1870'). Symbolae mycologicae. Beiträge zur Kenntnis der rheinischen Pilze. Jahrb. Nassauischen Vereins Naturk. 23-24, 1-459.

Fuckel L. 1873 ('1873 \& 1874'). Symbolae mycologicae. Zweiter Nachtrag. Jahrb. Nassauischen Vereins Naturk. 27-28, 1-99.

Fuckel L. 1876 ('1876 \& 1877'). Symbolae mycologicae. Dritter Nachtrag. Jahrb. Nassauischen Vereins Naturk. 29-30, 1-39.

Geschele EE. 1927. (The smuts in the vicinity of Sinelnikovo). Mater. Mikol. Fitopatol. 6, 92-95 (In Russian). 
Goebel F. 1830. Chemische Untersuchung einer in Persien herabgeregneten Substanz, der Parmelia esculenta. J. Chem. Phys. 60, 393-399.

Golovin PN. 1950. Novye vidy gribov Srednei Azii. Sredneaz. Gosud. Univ., N.S., Vyp. XIV, Biol. Nauk., Kniga 5, 1-47, Tashkent.

Golovin PN. 1952. Novye vidy golovnevykh gribov (Species novae ustilaginalium). Bot. Mater. Otd. Sporov. Rast. Bot. Inst. Komarova Akad. Nauk S.S.S.R. 8, 107-111.

González Fragoso R. 1916. Pugillus mycetorum Persiae (Lecti Ferd. Martinez de la Escalera). Bol. Real Soc. Esp. Hist. Nat. 16, 167-174.

González Fragoso R. 1919. Anotaciones micológicas. Mem. Real Soc. Esp. Hist. Nat. 11, 77-123.

González Fragoso R. 1926. Hongos de España. Broteria, Ser. Bot. 22, 97-106.

Gram E, Weber A. 1942. Växtsjukdomar utökad och bearbetad för svenska förhållanden av Carl Hammarlund. Stockholm, Lindfors Bokförlag AB. 629 pp.

Griffiths D. 1902. Concerning some West American fungi. Bull. Torrey Bot. Club 29, 290-301.

Guo L. 1990. The genera Sorosporium and Sporisorium in China. Mycosystema 3, 67-88.

Guo L. 2006a. Six new species of Anthracoidea (Ustilaginales) from China. Fungal Diversity 21, $81-92$.

Guo L. 2006b. Ustilago duriusculae, a new species of Ustilaginaceae (Ustilaginales) from China. Mycosystema 25, 161-162.

Gutner LS. 1941. Golovnevye griby. (The smut fungi). Lenin Acad. Agric. Sci. Inst. Plant Protection. Moskow \& Leningrad. 383 pp.

Gutner LS, Sergeeva KS. 1941. Novyj vid golovnevogo griba Ustilago kazachstanica (Ustilago kazachstanica species nova). Bot. Mater. Otd. Sporov. Rast. Bot. Inst. Akad. Nauk S.S.S.R. 5, 75-77.

Guyot L, Malençon G, Massenot M. 1958. Deuxième contribution à l'étude des Ustilaginales parasites du Bassin Méditerranéen Occidental (Afrique du Nord et Péninsule ibérique). Rev. Pathol. Vég. Entomol. Agric. France 37, 187-196.

Guyot L, Malençon G, Massenot M. 1960. Troisième contribution à l'étude des Ustilaginales parasites du Bassin Méditerranéen Occidental (Afrique du Nord et Péninsule ibérique). Rev. Pathol. Vég. Entomol. Agric. France 39, 163-172.

Guyot L, Malençon G, Massenot M. 1969. Quatrième contribution à l'étude des Ustilaginales parasites du Bassin Méditerranéen Occidental (Afrique du Nord, Espagne, Italie). Rev. Mycol. (Paris) 34, 192-219.

Hariot P. 1896. Note sur deux nouveaux champignons de France. J. Bot. (Morot) 10, 299-301.

Hariot P, Patouillard N. 1904. Description de champignons nouveau de l'Herbier du Muséum. Bull. Soc. Mycol. France 20, 61-65.

Hariot P, Patouillard N. 1909. Collections recueillies par M. A. Chevalier au Congo Français. Les Champignons de la région Chari-Tchad. Bull. Mus. Hist. Nat. (Paris) 15, 196-202.

Hedjaroude GHA, Abbasi M. 2000. Study of micromycetes flora in Karaj region, Iran (1). Rostaniha $1,103-130$.

Henderson DM. 1955. Urocystis floccosa in Britain. Notes Roy. Bot. Gard. Edinburgh 21, 241-242.

Hennings P. 1892. Fungi africani. Bot. Jahrb. Syst. 14, 337-373.

Hennings P. 1893a. Fungi africani, II. Bot. Jahrb. Syst. 17, 1-42.

Hennings P. 1893b. Fungi aethiopico-arabici I. G. Schweinfurth legit. Bull. Herb. Boissier 1, 97122.

Hennings P. 1894. Ustilago tritici (Pers.) Jens. form. folicola P. Henn. Z. Pflanzenkrankh. 4, 139.

Hennings P. 1895. Fungi austro-africani. Hedwigia 34, 325-327.

Hennings P. 1896. Beiträge zur Pilzflora Südamerikas I. Myxomycetes, Phycomycetes, Ustilagineae und Uredineae. Hedwigia 35, 207-262.

Hennings P. 1902. Battareopsis artini n. gen., sowie andere von Professor Dr. G. Schweinfurth in Aegypten 1901-1902 gesammelte Pilze. Beibl. Hedwigia 41, (210)-(215).

Hennings P. 1904. Doassansia renkaufii P. Henn. n. sp. auf Hydrocharis morsus-ranae L. Hedwigia 43, 434. 
Hennings P. 1907. Fungi. In Wildeman de É., Flore du Bas- et du Moyen-Congo. Ann. Mus. Congo, Sér. 5, Bot. 2, 85-106.

Hirschhorn E. 1939a. Una nueva especie de Ustilago de la flora Argentina. Notas Mus. La Plata, Bot. 4, 415-419.

Hirschhorn E. 1939b. Las especies de Cintractia de la flora Argentina. Revista Argent. Agron. 6, 179-202 + Pls. IV-VII.

Hirschhorn E. 1941. Las especies de Sorosporium de la flora Argentina. Revista Mus. La Plata, N.S., Secc. Bot. 3, 335-354 + Pls. I-IV.

Hirschhorn E. 1986. Las Ustilaginales de la flora Argentina. La Plata, CIC, 530 pp.

Hopkins JCF. 1938. A preliminary list of Rhodesian fungi. Trans. Rhodesia Sci. Assoc. 35, 97-127.

Hume HH. 1902. Ustilaginae of Iowa. Proc. Iowa Acad. Sci. 9, 226-240.

Ito S. 1935. Notae mycologicae Asiae orientalis II. Trans. Sapporo Nat. Hist. Soc. 14, 87-96.

Ito S. 1936. Mycological Flora of Japan. Vol. 2, Basidiomycetes. No. 1. Ustilaginales. Tokyo, Yokendo, $148 \mathrm{pp}$.

Ito S, Homma Y. 1938. Notae Mycologicae Asiae Orientalis III. Trans. Sapporo Nat. Hist. Soc. 15, $113-128$.

Jaczewski AA. 1893. Quelques champignons récoltés en Algérie. Bull. Soc. Mycol. France 9, 46-51 + Pl. 3.

Jaczewski AA. 1925. (New smut fungus on rye). Izv. Gosud. Inst. Opyt. Agron. 3, 106-109 (In Russian).

Jensen JL. 1888. Kornsortens brand (Anden meddelelse), etc. (In Danish). Kjøbenhavn. 1888, 72 pp.

Johanson CJ. 1884. Svampar från Island. Öfvers. Förh. Kongl. Svenska Vetensk.-Akad. 41(9), 157174.

Jørstad I. 1960. Iranian plants collected by Per Wendelbo in 1959. II. Uredinales and some other parasitic fungi. Acta Univ. Bergensis, Ser. Math. Rer. Naturalium, 1960, 11, 1-33.

Kakishima M, Ono Y. 1993. Smut fungi (Ustilaginales) collected in Pakistan. In Nakaike T. \& Malik S. (eds). Cryprogamic flora of Pakistan, 2, 187-214.

Kalchbrenner C, Cooke MC. 1880. South African Fungi. Grevillea 9, 17-34.

Karampour F, Asadpour R, Farrar N, Ershad D. 2008. Occurrence of stalk smut and inflorescence loose smut of grasses in Hormozgan. Proceedings of the 18th Iranian Plant Protection Congress, Hamedan, Iran. 2,636.

Karatygin IV, Azbukina ZM. 1989. Opredelitel' gribov SSSR. Porjadok Golovnevye. Vyp. 1. Semejstvo Ustilagovye (Definitorium fungorum URSS. Ordo Ustilaginales. Fasc. 1. Familia Ustilaginaceae). Nauka, Leningrad, 220 pp.

Karsten PA. 1884. Finlands rost- och brandsvampar (Hypodermii) i korthet beskrifna. Bidrag Kännedom Finlands Natur Folk 39, 1-118 (In Swedish).

Kawecka-Starmachowa B. 1939. Głownie i śniecie Polski. (Materiały do monografii). Czśęść II. Śniecie. (Brandpilze Polens. II. Teil. Tilletieae). Spraw. Komis. Fizjogr. 73, 147-223.

Kellerman WA, Swingle WT. 1890. Report on the loose smuts of cereals. Rep. (Annual), Kansas Agric. Exp. Sta. 2, 213-288 + 11 Pls.

Khabiri E. 1952. Contributions à la mycoflore de l' Iran. Première liste. Revue Mycol. 17, 154-157.

Khabiri E. 1956. Contribution à la mycoflore de l' Iran. Deuxième liste. Revue. Mycol. 21, 174-176.

Khabiri E. 1958. Contributions à la mycoflore de 1 Iran. Troisième liste. Revue Mycol. 23, 408-412.

Kitunen E. 1922. Untersuchungen über den Haferbrand und die Brandanfälligkeit der verschiedenen Hafersorten. Suomen Maanviljelys-taloudellinen Koelaitos. Tieteellisiä Julkaisuja 15, 1-127.

Kochman J. 1934. (Contribution to the knowledge of the Polish Ustilaginales). Acta Soc. Bot. Poloniae 11 (Suppl.), 285-303 + 3 Pls (In Polish).

Kochman J. 1936. Grzyby Głowniowe Polski. Ustilaginales Poloniae. Pl. Polon. 4, 1-161 + 12 Pls. (In Polish).

Körnicke F. 1877a. Mykologische Beiträge. Hedwigia 16, 17-31.

Körnicke F. 1877b. Mykologische Beiträge. Hedwigia 16, 33-40. 
Krishna A, Singh RA. 1980. A new smut fungus of Japanese barnyard millet. Indian J. Mycol. Pl. Pathol. 10, 177-178.

Kühn J. 1872. Die Brandformen der Sorghum-Arten, Tilletia sorghi Tulasne und Ustilago cruenta J. Kühn. Hamburger Garten- Blumenzeitung 28, 177-178.

Kühn J. 1873. In Rabenhorst, Repertorium. Hedwigia 12, 149-153.

Kühn J. 1874. Mittheilungen über die Entwickelungsformen des Getreidebrandes, etc. Ber. Sitzungen Naturf. Ges. Halle 1874, 5-7.

Kühn J. 1877. Die Brandformen der Sorghumarten. Mitth. Vereins Erdk. Halle 1877, 81-87.

Kukkonen I. 1963. Taxonomic studies on the genus Anthracoidea (Ustilaginales). Ann. Bot. Soc.Zool. Bot. Fenn. "Vanamo" 34, 1-122.

Kukkonen I. 1964. Type of germination and taxonomic position of the genus Anthracoidea. Trans. Brit. Mycol. Soc. 47, 273-280.

Kukkonen I, Ershad D. 2002. Two smuts new to Iran. Rostaniha 3, 113-114.

Kunze G, Schmidt JC. 1817. Neue Arten, etc. In Mykologische Hefte, etc. I. Leipzig.

Kunze J. 1830. Uredo (Ustilago) trichophora $\beta$ penniseti Kunze; Uredo (Ustilago) digitariae Kunze, n. spp. In Holl F. Verzeichnis der auf der Insel Madeira beobachteten Pflanzen, nebst Beschreibung einiger neuen Arten. Flora 13, 369-392.

Lagerheim G. 1889. Revision der im Exsiccat "Kryptogamen Badens von Jack, Leiner und Stizenberger" enthaltenen Chytridiaceen, Peronosporeen, Ustilagineen und Uredineen. Mitt. Bad. Bot. Vereins 1889, 69-76.

Lagerheim G. 1899. Contributions à la flore mycologique des environs de Montpellier. Bull. Soc. Myc. France 15, 95-103.

Langdon RFN. 1948. Records of Queensland fungi, V. Univ. Queensland Dept. Biol. Pap. 2(9), 1-9.

Langdon RFN, Fullerton RA. 1978. The genus Sphacelotheca (Ustilaginales): criteria for its delimitation and the consequences thereof. Mycotaxon 6, 421-456.

Lavrov NN. 1934. (Ustilagineae novae vel rarae Asiae septentrionalis). Trudy Tomsk. Gosud. Univ. $86,83-87$.

Lavrov NN. 1936. (Ustilaginaceae novae vel rarae Asiae borealis centralisque). Trudy Biol. NaučnoIssl. Inst. Tomsk. Gosud. Univ. 2, 1-35 (In Russian).

Lavrov NN. 1937. Tilletiaceae novae vel rarae Unionis Sovieticae. Sist. Zametki Mater. Gerb. Krylova Tomsk. Gosud. Univ. Kujbyševa 11, 1-4 (In Latin).

Lavrov NN. 1938. Redkie golovnevye griby sem. Tilletiaceae Evrazii (Tilletiaceae rarae eurasiaticae). Trudy Biol. Inst. Tomsk. Gosud. Univ. Kujbiševa 5, 43-85 (In Russian).

Lavrov NN. 1951. Flora gribov i slizevikov Sibiri i smezhnyh oblastei Evropy, Azii i Ameriki. Trudy Tomsk. Gosud. Univ., Ser. Biol. 110, 1-311 (In Russian).

Léveillé JH. 1846. Description des champignons de l'herbier de Muséum du Paris. Ann. Sci. Nat. Bot., Sér. 3, 5, 111-167; 249-304.

Léveillé JH. 1847. Sur la disposition méthodique des Urédinées. Ann. Sci. Nat. Bot., Sér. 3, 8, 369376.

Lindeberg B. 1959. Ustilaginales of Sweden (exclusive of the Cintractias on Caricoideae). Symb. Bot. Upsal. 16(2), 1-175.

Ling L. 1950. Taxonomic notes on Asiatic smuts. II. Sydowia 4, 74-81.

Ling L. 1951. Taxonomic notes on the Ustilaginales. Lloydia 14, 101-110.

Ling L. 1953. Taxonomic notes on the Ustilaginales. II. Lloydia 16, 180-192.

Link HF. 1825. Cryptogamia. Gymnomycetes. In Linné's Species plantarum. Ed. 4, 6(2), 1-128. Berolini, Impensis G.C. Nauk.

Liou TN. 1949. Sur l'existance d'une nouvelle famille des Ustilaginales. Contr. Inst. Bot. Natl. Acad. Peiping 6, 37-47.

Liro JI. (as 'Lidroth') 1904. Mykologische Mitteilungen 11-15. Acta Soc. Fauna Fl. Fenn. 26(5), 118. 
Liro JI. 1920. Kertomus kasvifysiologisen, -patologisen ja bakteriologisen osaston toiminnasta vuosina 1915-1916. Suomen Maanviljelys- taloudellinen Koelaitos. Vuosikirja 1915-1916. Helsinki, pp. 11-29 (In Finnish).

Liro JI. 1922. Über die Gattung Tuburcinia Fries. Ann. Univ. Fenn. Abo., Ser. A, 1(1), 1-153.

Liro JI. 1924. Die Ustilagineen Finnlands I. Ann. Acad. Sci. Fenn., Ser. A, 17(1), 1-636.

Liro JI. 1938. Die Ustilagineen Finnlands II. Ann. Acad. Sci. Fenn., Ser. A, 42(1), 1-720.

Liro JI. 1939. Mycotheca fennica. Die Etiketten. No. 301-600. Helsinki, 136 pp.

Lutz M, Göker M, Piatek M, Kemler M, Begerow D, Oberwinkler F. 2005. Anther smuts of Caryophyllaceae: molecular characters indicate host-dependent species delimitation. Mycol. Progr. 4, 225-238.

Magnus P. 1875. Mykologische Mittheilungen. Hedwigia 14, 17-21.

Magnus P. 1890. Über eine neue in den Fruchtknoten von Viola tricolor arvensis auftretende Urocystis-Art, Urocystis kmetiana. Verh. Bot. Vereins Prov. Brandenburg 31, XIX-XX.

Magnus P. 1893. Zwei in Persien neu aufgefundene Pilze. Verhandlungen der Gesellschaft deutscher Naturforscher und Ärzte. 65, 151-152.

Magnus P. 1894. Die von J. Peyritsch in Tirol gesammelten und im Herbarium der k. k. Universität zu Innsbruck aufbewahrten Pilze. Ber. Naturwiss.-Med. Vereins Innsbruck 21, 25-73 + Pl.

Magnus P. 1896a('1895'). Die Ustilagineen (Brandpilze) der Provinz Brandenburg. Verh. Bot. Vereins Prov. Brandenburg 37, 66-97 + Pl. I.

Magnus P. 1896b. Berichtigung zur Cintractia seymouriana P. Magnus. Ber. Deutsch. Bot. Ges. 14, 391-392.

Magnus P. 1899. J. Bornmüller, Iter Persico-turcicum 1892/93. Fungi, Pars II. Ein Beitrag zur Kenntniss der Pilze des Orients. Verh. K. K. Zool.-Bot. Ges. Wien 49, 87-103 + Pl. II.

Magnus P. 1903. J. Bornmüller, Iter anatolicum tertium 1899. - Fungi. Ein weiterer Beitrag zur Kenntniss der Pilze des Orients. Bull. Herb. Boissier, Sér. 2, 3, 573-587 + 2 Pls.

Maire R. 1906. Contributions à l'étude de la flore mycologique de l'Afrique du Nord. Bull. Soc. Bot. France 53, CLXXX-CCXV.

Maire R. 1917. Champignons nord-africains nouveaux ou peu connus. Bull. Soc. Hist. Nat. Afrique N. 8, 134-200.

Maire R. 1919. Une Ustilaginale nouvelle de la flore nord-africaine. Bull. Soc. Hist. Nat. Afrique N. $10,46-47$.

Maire R. 1931a. Champignons nord-africains nouveaux ou peu connus. Fascicule 5. Bull. Soc. Hist. Nat. Afrique N. 22, 13-24.

Maire R. 1931b. Champignons parasites africains nouveaux ou peu connus. - Recueil Trav. Cryptog. déd. Louis Mangin. Paris, pp. 355-360 + Pl. 26.

Massenot M. 1953. A propos d'une nouvelle espèce de Tuburcinia parasite d'Allium vineale. Rev. Mycol. (Paris), N.S., 18, 49-55.

Mayor E, Terrier C. 1959. Nouvelles excursions mycologiques en Corse. Rev. Mycol. (Paris), N.S., 24, 386-418.

McAlpine D. 1895. Australian fungi. - Proc. Roy. Soc. Victoria, N.S., 7, 214-221.

McAlpine D. 1910. The Smuts of Australia. Their structure, life history, treatment, and classification. Melbourne, $285 \mathrm{pp}$.

Mehrian F. 1984. Occurrence of maize common smut in Iran (in Farsi). Iranian J. Pl. Pathol. 20, 4650.

Mérat FV. 1821. Nouvelle flore des environs de Paris, etc. Ed. 2. Vol. 1. Paris.

Meyen FJF. 1841. Pflanzenpathologie. Berlin, Haude \& Spener, 330 pp.

Mitra M. 1931. A new bunt of wheat in India. Ann. Appl. Biol. 18, 178-180.

Moesz G. 1942. Budapest és környékének gombái. (Die Pilze von Budapest und seiner Umgebung). Budapest, 320 pp.

Moesz G. 1950. A Kárpát-medence üszöggombái. (Les Ustilaginales du Bassin des Carpathes). Budapest, Egyetemi Könyvkiadó, 256 pp. 
Montagne JFC. 1856. Sylloge generum speciorumque cryptogamarum, etc. Paris, J.B. Baillière, 498 pp.

Moore RT. 1992. The genus Bauhinus gen. nov.: for species of Ustilago on dicot hosts. Mycotaxon 45, 97-100.

Morini F. 1884. Di una nuova Ustilaginea. Mem. Reale Accad. Sci. Ist. Bologna, Ser. 4, 5, 794-804.

Mundkur BB. 1939a. A contribution towards a knowledge of Indian Ustilaginales. Trans. Brit. Mycol. Soc. 23, 86-121.

Mundkur BB. 1939b. Taxonomy of the sugarcane smuts Kew Bull. 10, 525-533

Mundkur BB. 1940. A second contribution towards a knowledge of Indian Ustilaginales. Trans. Brit. Mycol. Soc. 24, 312-336.

Mundkur BB. 1943. Studies in Indian cereal smuts. VI. The smuts on sawan (Echinochloa frumentacea). Indian J. Agric. Sci. 13, 631-633.

Mundkur BB. 1944a. Some rare and new smuts from India. Indian J. Agric. Sci. 14, 49-52.

Mundkur BB. 1944b. Fungi of the Northwestern Himalayas: Ustilaginales. Mycologia 36, 286-293.

Nannfeldt JA. 1979. Anthracoidea (Ustilaginales) on Nordic Cyperaceae-Caricoideae, a concluding synopsis. Acta Univ. Upsal., Symb. Bot. Upsal. 22(3), 1-41.

Nees v. Esenbeck CG. 1817. Das System der Pilze und Schwämme. Würzburg. XXXVIII + 329 pp.

Niemann E, Bamdadian A. 1967. Die Ustilago-Brande der Gerste in Iran. Entomol. Phytopathol. Appliquées 25, 7-9.

Niessl G. 1861. Beitrag zur Kryptogamenflora von Niederösterreich. Oesterr. Bot. Z. 11, 324-329.

Niessl G. 1869-72. Beiträge zur Kenntniss der Pilze. Beschreibungen neuer und wenig bekannter Pilze. Verh. Naturf. Vereins Brünn 10, 153-217 + Pls. III-VII.

Niessl G. 1876. Mycologische Notizen. Hedwigia 15, 1-2.

Opiz PM. 1823-25. Naturalientausch. No. 1-10. Prag. Sechstes Verzeichniss 1924, 37-52.

Orbigny de ACVD. 1849. Dictionnaire universel d'histoire naturelle. Vol. 12. Edit. MM Renard, Martinet et Co., Paris.

Oudemans CAJA. 1866. Prodromus Florae Bataviae, Ed. 2, 4. (Fungi). Amsterdam. (n.v.).

Padwick GW. 1946. Notes on Indian fungi. IV. Mycol. Pap. 17, 1-12.

Pascoe IG, Crump N, Jones RH. 2005. Tilletia contraversa isolates on Critesion spp. (barley grasses) in Australia are not conspecific with $T$. contraversa isolates that cause dwarf bunt of wheat. The $15^{\text {th }}$ Biennial Australasian Plant Pathology Society Conference handbook, p. 289.

Passerini G. 1876. A new "Tilletia". Grevillea 5, 47.

Passerini G. 1877. Funghi Parmensi. Nuovo Giorn. Bot. Ital. 9, 235-267.

Patouillard N. 1903. Additions au catalogue des champignons de la Tunisie. Bull. Soc. Myc. France 19, 245-261.

Patouillard N, Hariot P. 1900. Énumération des champignons récoltés par M.A. Chevalier au Sénégal et dans le Soudan occidental. J. Bot. (Morot) 14, 234-244.

Peck CH. 1873. Description of new species of fungi. Bull. Buffalo Soc. Nat. Sci. 1, 41-72.

Peck CH. 1882. New species of fungi. Bot. Gaz. (Crawfordsville) 7, 54-57.

Persoon CH. 1797. Tentamen dispositionis methodicae fungorum. Lipsiae. 76 pp, 4 Pls.

Persoon CH. 1801. Synopsis methodica fungorum. Pars I \& II. Gottingae, Dietrich. XXX + 706 pp. +5 Pls.

Petch T. 1924. [Gymnosporium lineare B. \& Br.]. Ann. Roy. Bot. Gard. (Peradeniya) 9, 166-167.

Petrak F, Esfandiari E. 1941. Beiträge zur Kenntnis der naturhistorischen Pilzflora. Ann. Mycol. 39, 204-228.

Picbauer R. 1932. Fungi a Doc. Dr. Fr. Nábělek in itinere turcico-persico lecti. Práce Morav. Př́rr. Společn. 7(11), 1-4.

Piepenbring M, Stoll M, Oberwinkler F. 2002. The generic position of Ustilago maydis, Ustilago scitaminea, and Ustilago esculenta (Ustilaginales). Mycol. Progr. 1, 71-80.

Plowright CB. 1889. A monograph of the British Uredineae and Ustilagineae. London, Kegan Pauls Trench \& Co., 347 pp.

Poiret JL. 1808. Encyclopédie méthodique. Botanique. Vol. 8. Paris, H. Agasse. 
Pole-Evans IB. 1916. A new smut on Sorghum halepense Nees. S. African J. Sci. 12, 534-544.

Potter AA. 1912. Ustilagineae on Andropogon sorghum (L.) in America. Phytopathology 2, 98.

Pourjam E. 1989. Occurrence of Tilletia triticoides in Iran. Abstract. Proceedings of the Ninth Plant Protection Congress of Iran, p. 126.

Preuss CG. 1848. Uredo agropyri Preuss. In Sturm J., Deutschlands Flora 3, 1-2.

Prillinger H, Deml G, Dörfler Ch, Laaser G, Lockau W. 1991. Ein Beitrag zur Systematik und Entwicklungsbiologie höherer Pilze: Hefe-Typen der Basidiomyceten. Teil II: Microbotryum-Typ. Bot. Acta 104, 5-17.

Rabenhorst L. 1851. Klotzschii Herbarium vivum mycologicum, etc. Centuria XV. Flora 9, $72-77$.

Rabenhorst L. 1855. Klotzschii Herbarium vivum mycologicum, etc. Cent. XX. Bot. Zeitung (Berlin) 13, 299-302.

Rabenhorst L. 1857. Klotzschii Herbarium vivum mycologicum, etc. Ed. nova. Centuria IV. Bot. Zeitung (Berlin) 15, 93-96.

Rabenhorst L. 1866. Fungi europaei exsiccati, etc. Ed. nova. Cent. XI. Bot. Zeitung (Berlin) 24, 411-412.

Rabenhorst L. 1871. Uebersicht der von Herrn Prof. Dr. Haussknecht im Orient gesammelten Krytogamen. Hedwigia 10, 17-27.

Rabenhorst L. 1874. Fungi europaei exs. Cent. XIX. Nr. 1801-1900. Hedwigia 13, 174-175 \& 184189.

Rabenhorst L. 1876. Fungi europaei, Cent. 21 und 22. Hedwigia 15, 103-110 \& 116-121.

Rabenhorst L. 1881. Kryptogamen-Flora von Deutschland, Oesterreich und der Schweiz. 2. Aufl., 1. Pilze, I. Abt., pp. 79-131.

Ranojević N. 1914. Dritter Beitrag zur Pilzflora Serbiens. Ann. Mycol. 12, 393-421.

Reichert I. 1921. Die Pilzflora Ägyptens. Eine mykogeographische Studie. Bot. Jahrb. Syst. 56, 598-728. + Pls. II-IV.

Ricker PL. 1905. Notes on fungi II. With new species from various localities. J. Mycol. 11, 111115.

Rivolta S. 1873. Dei parassiti vegetali come introduzione allo studio delle malattie parassitarie e delle alterazioni dellalimento degli animali domestici. Torino, $592 \mathrm{pp}$.

Rostrup E. 1889. Oversigt over de i 1888 indløbne forespørgsler angaaende sygdomme hos kulturplanter. Tidsskr. Landoekon., Ser. 5, 8, 744-751.

Rostrup E. 1890a. Ustilagineae Daniae. Danmarks Brandsvampe. Festskr. Bot. Foren. Kjøbenhavn. 1890, 115-168.

Rostrup E. 1890b. Nogle undersøgelser angaaende Ustilago carbo. Overs. Kongel. Danske Vidensk. Selsk. Forh. Medlemmers Arbeider 1890, 1-16 + Pl. I.

Roumeguère C, Saccardo P. 1881. Reliquiae mycologicae Libertianae. Series altera. Rev. Mycol. (Toulouse) 3, 39-59+2 Pls.

Roussel HFA. 1806. Flore du Calvados et des terreins adjacens. Ed. 2. Caen.

Saccardo PA. 1877. Fungi veneti novi vel critici, etc. Michelia 1, 1-72.

Saccardo PA. 1879. Fungi veneti novi v. critici, etc. Ser. X. Michelia 1, 539-546.

Saccardo PA. 1888. Ustilagineae Tul. (by J.B. de Tony). In Sylloge fungorum, etc. 7, 449A-498A, 499-527.

Saccardo PA. 1908. Notae mycologicae. Series X. Ann. Mycol. 6, 553-569 + Pl. XXIV.

Saccardo PA. 1915. Fungi ex insula Melita (Malta) lecti a Doct. A. Caruana-Gatto et Doct. G. Borg annis MCMXII et MCMXIV. Series III. Nuovo Giorn. Bot. Ital., N.S., 22, 24-76.

Saccardo PA, Trotter A. 1913. Fungi Tripolitani. Ann. Mycol. 11, 409-420.

Savile DBO. 1952. A study of the species of Cintractia on Carex, Kobresia, and Scirpus in North America. Canad. J. Bot. 30, 410-435.

Săvulescu T. 1951. Noutăţi din micoflora R.P. Române. Bul. Şti. Acad. Republ. Populare Române 3, 211-227. 
Săvulescu T. 1956. Die physiologischen Rassen von Tilletia foetida (Bauer) Liro und die den Steinbrand hervorrufenden Tilletia-Arten in der Rumänischen Volksrepublik. Phytopathol. Z. 25, 267-310.

Săvulescu T. 1957. Ustilaginalele din R.P. Romînă. I-II. Bucureşti, Edit. Acad., 1168 pp.

Scharif G, Ershad D. 1966. A list of fungi on cultivated plants, shrubs and trees of Iran. Ministry of Agriculture, Plant Pests and Diseases Resrach Institute, Evin-Tehran, 89+3 pp., one map.

Schellenberg HC. 1911. Die Brandpilze der Schweiz. Beitr. Kryptogamenfl. Schweiz 3(2), XLVI + $1-180$.

Schellenberg HC. 1915. Ein neuer Brandpilz auf Arrhenatherum elatius (L.) M. u. K. Ber. Deutsch. Bot. Ges. 33, 316-323 + Pl. VII.

Schlechtendal DFL. 1824. Flora Berolinensis, Pars 2. Cryptogamia. Berlin. XIV + 284 pp.

Schlechtendal DFL. 1826. Fungorum novorum et descriptorum illustrationes. Linnaea 1, 235-249.

Schmidt JC, Kunze G. 1819. Deutschlands Schwämme in getrockneten Exemplaren. Lief. 9. Leipzig.

Schmiedeknecht M, Puncag T. 1966. Ustilaginales aus der Mongolischen Volksrepublik. Feddes Repert. 73, 170-183.

Schneider WG. 1869. Ueber einige für die schlesische Pilzflora neue Funde. Jahresber. Schles. Ges. Vaterl. Cult. 46, 142.

Scholz H, Scholz I. 1988. Die Brandpilze Deutschlands (Ustilaginales). Englera 8, 1-691.

Schrank FP. 1793. Beschreibung einiger Cryptogamisten. In Hoppes Botanisches Taschenbuch a. d. Jahr 1793. Regensburg 1793, pp. 60-69.

Schröter J. 1872('1869'). Die Brand- und Rostpilze Schlesiens. Abh. Schles. Ges. Vaterl. Cult., Abth. Naturwiss.1869/72, 1-31.

Schröter J. 1877. Bemerkungen und Beobachtungen über einige Ustilagineen. In Cohn F., Beiträge zur Biologie der Pflanzen 2, 349-385 + PI. XII. Breslau.

Schröter J. 1887. Ustilaginei. In Cohn, Kryptogamen-Flora von Schlesien 3(1), 261-291. J. U. Kerns Verlag, Breslau.

Schwarzman SR. 1960. Golovnevye griby. (Smut fungi). Flora sporovych rastenij Kazachstana 2. Alma Ata. 369 pp. (In Russian).

Schweinitz LD. de 1822. Synopsis fungorum Carolinae superioris. Schriften Naturf. Ges. Leipzig 1, 20-131.

Sharifnabi B, Hedjaroude GA. 1993. Contribution to knowledge of smuts of Iran. Iranian J. Pl. Pathol. 29, 41-42.

Smarods J. 1963. Fungi latvici exsiccati. Latv. Vals. Univ. Zināt. 49, Ser. Biol. 2, 107-156.

Sorokin N. 1890. Matériaux pour la flore cryptogamique de l'Asie Centrale. Rev. Mycol. (Toulouse) 12, 207-208.

Sousa da Camara ME. De, Oliveira ALB. 1945. Contributio fungorum minima in Lusitania collectorum. Ustilaginales. I. Agron. Lusit. 7, 101-108.

Sowerby J. 1796-1803. Coloured figures of English fungi or mushrooms. Vols. 1-3 + Supl. 18091815. (440 Tabs.). London.

Spegazzini C. 1880. Fungi argentini (Continuacion). Anales Soc. Ci. Argent. 10, 5-33.

Spegazzini C. 1881. Fungi argentini additis nonnullis brasiliensibus montevideensibusque. Anales Soc. Ci. Argent. 12, 63-82.

Spegazzini C. 1899. Fungi argentini novi vel critici. Anales Mus. Nac. Buenos Aires, Ser. 2, 6, 81$367+2$ Pls.

Spegazzini C. 1902. Mycetes argentinenses (Series II). Anales Mus. Nac. Buenos Aires, Ser. 3, 1, 47-89.

Spegazzini C. 1909. Mycetes argentinenses (Series IV). Anales Mus. Nac. Buenos Aires, Ser. 3, 12, 257-458.

Spegazzini C. 1912. Mycetes argentinenses (Series VI). Anales Mus. Nac. Hist. Nat. Buenos Aires 23, 1-146. 
Spegazzini C. 1915. Fungi nonnulli senegalenses et canarienses lecti et descripti a Spegazzini Carolo. Anales Mus. Nac. Hist. Nat. Buenos Aires 26, 117-134.

Spegazzini C. 1925. Ustilagineas Argentinas nuevas o críticas. Revista Argent. Bot. 1, 145-156.

Sternon F. 1925. L'hétérogénéité du genre Ramularia. Thèse, 99 pp., Nancy.

Stevenson JA, Johnson AG. 1944. The nomenclature of the broomcorn millet smut fungus. Phytopathology 34, 613.

Sturm J. 1816. Deutschlands Flora, etc. Abt. III, Heft 3. Ditmar, L.P.F. Die Pilze Deutschlands. Nürnberg.

Sturm J. 1853. Deutschlands Flora, etc. Abt. III, Heft 33 \& 34. Strauss, F. Die Pilze Deutschlands. Nürnberg.

Sydow H. 1924. Notizen über Ustilagineen. Ann. Mycol. 22, 277-291.

Sydow H. 1935. Beschreibungen neuer südafrikanischer Pilze. VI. Ann. Mycol. 33, 230-237.

Sydow H. 1939. Novae fungorum species. XXVII. Ann. Mycol. 37, 197-253.

Sydow H, Ahmad S. 1939. Fungi panjabenses. Ann. Mycol. 37, 439-447.

Sydow H, Sydow P. 1901. Zur Pilzflora Tirols. Oesterr. Bot. Z. 51, 11-29.

Sydow H, Sydow P. 1908. Micromycetes orientales a cl. J. Bornmüller communicati. Annales Mycologici 6, 526-530.

Sydow H, Sydow P. 1912. Einige neue parasitische Pilze aus Russland. Ann. Mycol. 10, 214-217.

Sydow H, Sydow P. 1919. Mykologische Mitteilungen. Ann. Mycol. 17, 33-47.

Sydow H, Sydow P, Butler EJ. 1906. Fungi Indiae orientalis. Pars I. Ann. Mycol. 4, 424-445.

Sydow H, Sydow P, Butler EJ. 1912. Fungi Indiae orientalis. Pars IV. Ann. Mycol. 10, 243-280.

Takahashi Y. 1902. On Ustilago panici-miliacei (Pers.) Winter. Bot. Mag. (Tokyo) 16, 247-257 (in Japanese) \& 183-185 (English summary) + Pl. I.

Tapke VF. 1932. An undescribed loose smut of barley. Phytopathology 22, 869-870.

Thirumalachar MJ, Mundkur BB. 1951. Revisions of and additions to Indian fungi. III. Mycol. Pap. $40,1-15$.

Thirumalachar MJ, Neergaard P. 1978('1977'). Studies on the genus Tolyposporium. Tolyposporidium gen. nov. Friesia 11, 177-192.

Thirumalachar MJ, Pavgi MS. 1956. Notes on Indian Ustilagineae. VI. Mycopathol. Mycol. Appl. 7, 282-290 + Pls. I-II.

Thümen von F. 1873. Mycologische Notizen. Hedwigia 12, 113-115.

Thümen von F. 1874. Verzeichnis der in der Umgegend von Krems in Nieder-Oesterreich gesammelten Pilze. Verh. K. K. Zool.-Bot. Ges. Wien 24, 483-494.

Thümen von F. 1879. Fungi Egyptiaci. Ser. II. Grevillea 8, 49-51.

Thümen von F. 1880. Pilze aus Entre-Rios. Flora 63, 30-31.

Torabi M, Mardoukhi V, Jaliani N. 199). First report on the occurrence of partial bunt on wheat in the Southern parts of Iran. Seed and Plant 12, 8-9.

Tracy SM, Earle FS. 1895. New species of parasitic fungi. Bull. Torrey Bot. Club 22, 174-179.

Tracy SM, Earle FS. 1896. New species of fungi from Mississippi. Bull. Torrey Bot. Club 23, 205211.

Tracy SM, Earle FS. 1899. New fungi from Mississippi. Bull. Torrey Bot. Club 26, 493-495.

Tulasne L-R, Tulasne C. 1847. Mémoire sur les Ustilaginées comparées aux Urédinées. Ann. Sci. Nat. Bot., Sér. 3, 7, 12-127 + Pls. 2-7.

Ule E. 1884. Beitrag zur Kenntnis der Ustilagineen. Verhandl. Bot. Vereins Prov. Brandenburg 25, 212-217.

Uljanishchev VI. 1950. (New and rare species of smut fungi collected in Azerbaydzhan). Trudy Inst. Bot. (Baku) 15, 74-107.

Uljanishchev VI. 1968. Novyi vid golovnevogo griba (A new species of Ustilaginales). Novosti Sist. Nizsh. Rast. 1968, 144-145.

Unamuno LM. 1940. Notas micológicas, Ser. 2, 1. Nueva aportacion al estudio de los hongos microscopicos de la zona del Protectorado Español en Marruecos. Mauritania 1940, 102-103 \& 132-134. 
Unger F. 1833. Die Exantheme der Pflanzen, etc. Wien, K. Gerold, 422 pp.

Unger F. 1836. Ueber den Einfluss des Bodens, etc. Wien, Rohrmann \& Schweigerd, XXIV + 367 pp.

Vánky K. 1977. Moesziomyces, a new genus of Ustilaginales. Bot. Not. 130, 131-135.

Vánky K. 1979. Species concept in Anthracoidea (Ustilaginales) and some new species. Bot. Not. 132, 221-231.

Vánky K. 1982. Ustilaginales. Fasc. XIV-XV (No. 326-375). Publ. Herb. Univ. Uppsala 9, 1-17.

Vánky K. 1983. Ustilaginales. Fasc. XVI-XVIII (No. 376-450). Publ. Herb. Univ. Uppsala 11, 125.

Vánky K. 1985a. Carpathian Ustilaginales. Acta Univ. Upsal., Symb. Bot. Upsal. 24(2), 1-309.

Vánky K. 1985b. K. Vánky, Ustilaginales exsiccata. Fasc. XIX-XX (No. 451-500). Publ. Herb. Ustilag. Vánky (HUV) 1, 1-17.

Vánky K. 1988a. Taxonomical studies on Ustilaginales. I. Mycotaxon 31, 401-406.

Vánky K. 1988b. Taxonomical studies on Ustilaginales. III. Mycotaxon 33, 365-374.

Vánky K. 1989. Taxonomical studies on Ustilaginales. IV. Mycotaxon 35, 153-158.

Vánky K. 1990. Taxonomical studies on Ustilaginales. VI. Mycotaxon 38, 267-278.

Vánky K. 2000. Taxonomical studies on Ustilaginales. XX. Mycotaxon 74, 161-215.

Vánky K. 2001. Taxonomical studies on Ustilaginales. XXI. Mycotaxon 78, 265-326.

Vánky K. 2003. Taxonomical studies on Ustilaginales. XXIII. Mycotaxon 85, 1-65.

Vánky K. 2004. Taxonomic studies on Ustilaginomycetes - 24. Mycotaxon 89, 55-118.

Vánky K. 2005. Taxonomic studies on Ustilaginomycetes - 25. Mycotaxon 91, 217-272.

Vánky K. 2007. Taxonomic studies on Ustilaginomycetes - 27. Mycotaxon 99, 1-70.

Vánky K. 2008. Taxonomic studies on Ustilaginomycetes - 28. Mycotaxon 106, 133-178.

Vánky K, Abbasi M. 2012a(2011). Anthracoidea songorica sp. nov. (Ustilaginomycetes) on Carex songorica (Cyperaceae) from Iran. Rostaniha 12(2), 181-185.

Vánky K, Abbasi M. 2012b(2011). Urocystis phalaridis sp. nov. (Urocystidaceae), on Phalaris sp. (Poaceae) from Iran. Rostaniha 12(2), 187-190.

Vánky K, Ershad D. 1993. Smut fungi (Ustilaginales) new to Iran. Iranian J. Pl. Pathol. 29, 1-22 (in Farsi) + 1-11 (in English).

Vánky K, Ershad D. 2002. A new Tilletia (Ustilaginomycetes) from Iran. Rostaniha 3, 19-24.

Vánky K, Lutz M. 2010. Entyloma majewskii. - Polish Bot. J. 55, 271-279.

Vánky K, McKenzie EHC. 2002. Smut fungi of New Zealand. Fungi of New Zealand, Vol. 2. Fungal Diversity Press, Hong Kong, 259 pp.

Vánky K, Scholz H. 2001. Three new species of smut fungi (Ustilaginomycetes). Nova Hedwigia 72, 391-398.

Vánky K, Abbasi M, Samadi S. 2011('2010'). Additions to the knowledge of smut fungi (Ustilaginomycetes) of Iran. Rostaniha 11, 191-198.

Vánky K, Deml G, Oberwinkler F. 1988. The smut fungi of Hyparrhenia hirta (Gramineae). J. Phytopathology 121, 181-191.

Vestergren T. 1902. Verzeichnis nebst Diagnosen und kritischen Bemerkungen zu meinem Exsiccatenwerke "Micromycetes rariores selecti", Fasc. 11-17. Bot. Not. 1902, 113-128; $161-179$.

Viennot-Bourgin G. 1937. Deux Entyloma de l'Ile de Madère. Rev. Mycol. (Paris), N.S., 2, 118-124.

Viennot-Bourgin G. 1951. Notes mycologiques. Rev. Pathol. Vég. Entomol. Agric. France 30, 3553.

Viennot-Bourgin G. 1954('1953'). Notes mycologiques (III). Bull. Soc. Mycol. France 69, 332-342.

Viennot-Bourgin G. 1957. Trois Ustilaginales nouvelles de Guinée française. Bull. Soc. Bot. France 104, 266-275.

Viennot-Bourgin G. 1958. Contribution a la conaissance des champignons parasites de l'Iran. Ann. Épiphyt., N.S., 9, 97-210.

Viennot-Bourgin G. 1968. Micromycètes nouveaux récoltés en Iran. Bull. Soc. Mycol. France 84, 497-503. 
Viennot-Bourgin G, Scharif G, Eskandari F. 1969. Nouvelle contribution à la conaissance des micromycètes parasites en Iran. Entomol. Phytopathol. Appl. 28, 3-27.

Viennot-Bourgin G, Ale-Agha N, Ershad D. 1970. Les champignons parasites de l'Iran (Nouvelle contribution). Ann. Phytopathol. 2, 689-734.

Wagner F. 1950. Auftreten, Sporenkeimung und Infektion des Zwergsteinbrandes an Weizen. Z. Pflanzenbau Pflanzenschutz 1, 1-13.

Wallroth KFW. 1833. Flora Cryptogamica Germaniae. Pars II, Vol. 4. Norimbergae.

Wang YC. 1962. (Some new species and new combination of smut fungi). Acta Bot. Sinica 10, 133136.

Westendorp GD. 1851. Notice sur quelques cryptogames inédites ou nouvelles pour la flore belge. Bull. Acad. Roy. Sci. Belgique 18, 384-417 + Pl.

Wettstein R. 1885. Fungi in O. Stapf, Die botanischen Ergebnisse der polakschen expedition nach Persien im Jahre 1882. Denkschr. Akad. Wiss. Wien. 50, 1-4.

Wille N. 1893. Mycologiske notiser. II. Om nögen og daekket brand paa havre og byg. Bot. Not. 1893, 3-11.

Winter G. 1881. Die Pilze Deutschlands, Oesterreichs und der Schweiz. II. Ordnung. Ustilagineae. In Rabenhorst's Kryptogamen-Flora von Deutschland, Oesterreich und der Schweiz. 2. Aufl., 1. Pilze, I. Abt., pp. 79-131.

Winter G. 1883. Ueber einige nordamerikanische Pilze. Hedwigia 22, 129-131.

Wolff R. 1874. Der Brand des Getreides, seine Ursachen und seine Verhütung. Halle, Verl. Buchh. Waisenhauses, 51 pp. (n.v.).

Wróblewski A. 1914. Przyczynek do znajomoçi grzybów Podola. (Contribution à la connaissance des champignons de Podolie). Spraw. Komis. Fizjogr. 48, 3-15.

Yen WY. 1937a. Recherches systématiques, biologiques et cytologiques sur les Ustilaginées de Chine. Contr. Inst. Bot. Natl. Acad. Peiping 4, 159-207.

Yen WY. 1937b. Note sur quelques Ustilaginées marocaines. Rev. Mycol. (Paris), N.S., 2, 76-84.

Yen WY. 1938a. Ustilaginées d'Afrique recueillies par M. Murat. I. Un nouveau Sphacelotheca. Rev. Mycol. (Paris), N.S., 3, 7-13.

Yen WY. 1938b. Ustilaginées d'Afrique recueillies par M. Murat. II. Rev. Mycol. (Paris), N.S., 3, 85-90.

Zambettakis C. 1970. Recherches sur les Ustilaginales d'Afrique. Bull. Soc. Mycol. France 86, 305692.

Zambettakis C. 1980('1979'). Recherches sur les Ustilaginales. (Supplément sur les espèces africaines). Bull. Soc. Mycol. France 95, 393-443.

Zaprometov NG. 1926. Materialy po mikoflore Srednei Azii. Vypusk 1-i. (Materials for the mycoflora of Central Asia. Part 1). Tashkent, 1-36 pp. (In Russian).

Zaprometov NG. 1928. Materialy po mikoflore Srednei Azii. Vypusk 2-i. (Materials for the mycoflora of Central Asia. Part 2). Tashkent, 70 pp. (In Russian).

Zogg H. 1986('1985'). Die Brandpilze Mitteleuropas unter besonderer Berücksichtigung der Schweiz. Cryptogamica Helvetica 16, 1-277.

Zundel GL. 1930a. Notes on Pennsylvania Ustilaginales. I. Mycologia 22, 97-100.

Zundel GL. 1930b. Monographic studies on the Ustilaginales attacking Andropogon. Mycologia 22, 125-158.

Zundel GL. 1933. New and rare North and South American Ustilaginales. Mycologia 25, 349-355.

Zundel GL. 1937. Miscellaneous notes on the Ustilaginales. Mycologia 29, 583-591.

Zundel GL. 1939. Studies on the Ustilaginales of the world. Mycologia 31, 572-589.

Zundel GL. 1942. Studies on the Ustilaginales of the world. II. Mycologia 34, 123-127.

Zundel GL. 1944. Notes on the Ustilaginales of the world. IV. Mycologia 36, 400-412.

Zundel GL. 1953. The Ustilaginales of the world. Contr. Dept. Bot. School Agric. Pennsylvania State Coll. 176, XI + 1-410. 